\title{
Grafos e hipergrafos com cintura e número cromático grandes
}

\author{
Giulia Satiko Maesaka
}

\author{
DisSERTAÇÃO APRESENTADA \\ $\mathrm{AO}$ \\ Instituto De Matemática e EstatísticA \\ DA \\ Universidade DE SÃo PAUlo \\ PARA \\ OBTENÇÃO DO TÍTULO \\ DE \\ Mestre em CiÊnCIAS \\ Programa: Ciência da Computação \\ Orientador: Prof. Dr. Yoshiharu Kohayakawa
}

Durante o desenvolvimento deste trabalho a autora recebeu auxílio financeiro da CNPq

São Paulo, abril de 2018 


\section{Agradecimentos}

Agradeço

1. aos amigos do Ciências Moleculares, por amarem a ciência;

2. aos amigos do IME, por serem compreensivos;

3. aos amigos que estudam coisas parecidas, por compartilharem problemas;

4. aos amigos da vida, por me abrirem o horizonte;

5. à minha família, incluindo o Mynghau, por me darem conforto;

6. ao Yoshi, por me orientar (o que me faz achar que acredita em mim);

7. ao Mota e ao Maurício, por terem sido uma banca bacana e pelos comentários pertinentes;

8. ao Rangel, a quem eu mostrei todas as contas e demonstrações dessa dissertação;

9. aos professores da Computação do IME, por serem inspiradores;

10. aos lugares de São Paulo, especialmente à USP do Butantã, por suas tomadas, café e por me permitirem caminhadas agradáveis e interessantes;

11. a quem por ventura vier a ler esse texto (isso faria tudo fazer mais sentido);

12. ao acaso.

Muito Obrigada! 


\section{Resumo}

MAESAKA, G. S. Grafos e hipergrafos com cintura e número cromático grandes. 2018. 91f. Dissertação - Instituto de Matemática e Estatística, Universidade de São Paulo, São Paulo, 2018.

A demonstração feita por Erdős da existência de grafos com cintura e número cromático grandes é uma das primeiras aplicações do método probabilístico. Essa demonstração fornece um limite para o número de vértices de um grafo desse tipo, que é exponencial na cintura quando o número cromático é fixado. O foco deste texto, no entanto, são as construções determinísticas de grafos com cintura e número cromático grandes e os números de vértices dos grafos obtidos. As construções elementares conhecidas nos fornecem apenas grafos com um número Ackermanniano de vértices.

O texto começa com uma breve repetição das demonstrações probabilísticas da existência de grafos e hipergrafos com cintura e número cromático grandes. Depois, a busca por construções determinísticas é motivada apresentando-se algumas construções para o caso particular de grafos livres de triângulo e com número cromático grande. São construídos os grafos de Tutte, Zykov, Mycielski e Kneser, os grafos de shift e os de planos projetivos finitos. Os números de vértices dessas construções são computados e comparados. De fato, a construção a partir de planos projetivos finitos tem um número polinomial de vértices.

A parte principal do texto são as construções de grafos e hipergrafos com cintura e número cromático grandes. A primeira construção apresentada foi feita por Kř́žz. Ela foi a primeira construção para grafos com cintura e número cromático grandes que não envolvia hipergrafos. A segunda construção apresentada foi feita por Nešetřil e Rödl. Essa construção antecede a de Křriž. Ela utiliza a amalgamação entre grafos e hipergrafos para obter um hipergrafo uniforme com cintura e número cromático grandes. A terceira e última construção apresentada foi encontrada por Alon, Kostochka, Reiniger, West e Zhu. Essa construção consegue obter hipergrafos uniformes com cintura e número cromático grandes diretamente a partir de um grafo, que é uma certa árvore aumentada. Em particular, essa construção obtém grafos com cintura e número cromático grandes sem envolver hipergrafos. Os números de vértices dos hipergrafos obtidos por essas construções são computados e comparados.

Palavras-chave: cintura, número cromático, amálgama, árvore aumentada. 


\section{Abstract}

MAESAKA, G. S. Graphs and hypergraphs with high girth and high chromatic number. 2018. 91f. Thesis - Instituto de Matemática e Estatística, Universidade de São Paulo, São Paulo, 2018.

The proof by Erdôs of the existence of graphs with high girth and high chromatic number is one of the first applications of the probabilistic method. This proof gives a bound on the number of vertices of such graphs, which is exponential on the girth if the chromatic number is fixed. The focus of this text is however on the deterministic construction of graphs with high girth and high chromatic number and on the number of vertices of the obtained graphs. The elementary known constructions can only give us graphs with an Ackermannian number of vertices.

We begin by briefly repeating the probabilistic proofs of the existence of graphs and hypergraphs with high girth and high chromatic number. Then we motivate the search for deterministic constructions of such graphs by showing some constructions for the special case of triangle-free graphs with high chromatic number. We construct Tutte, Zykov, Mycielski and Kneser graphs, the shift graphs and graphs built from finite projective planes. We count and compare the number of vertices of the graphs obtained by each of these constructions. In fact, the construction based on finite projective planes gives us graphs with a polynomial number of vertices.

The main part of the text consists of constructions of graphs and hypergraphs with high girth and high chromatic number. The first construction we present is due to Kříž. This was the first construction to give graphs with high girth and high chromatic number without using hypergraphs. The second construction we present is due to Nešetřil and Rödl. This construction precedes the one by Křriž. It uses amalgamations between graphs and hypergraphs to obtain uniform hypergraphs with high girth and high chromatic number. The third and last construction we show was found by Alon, Kostochka, Reiniger, West and Zhu. This construction manages to build uniform hypergraphs with high girth and high chromatic number directly from a single graph, which is an augmentedtree. In particular, it constructs graphs with high girth and high chromatic number without using hypergraphs. We count and compare the number of vertices of the hypergraphs obtained by these constructions.

Keywords: girth, chromatic number, amalgamation, augmented tree. 


\section{Conteúdo}

Lista de Figuras $\quad$ xi

1 Introdução 1

2 Considerações iniciais $\quad 5$

2.1 Demonstrações probabilísticas . . . . . . . . . . . . . . . . . . . . 6

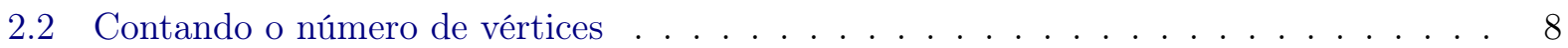

3 Grafos sem triângulos com número cromático grande $\quad 11$

3.1 O grafo de Tutte . . . . . . . . . . . . . . . . . . . . . . 11

3.2 O grafo de Zykov . . . . . . . . . . . . . . . . . . . . . . . 12

3.3 O grafo de Mycielski . . . . . . . . . . . . . . . . . . . . . . . 14

3.4 Os grafos de shift . . . . . . . . . . . . . . . . . . . . . 15

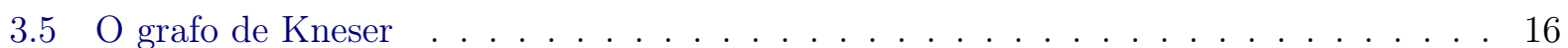

3.6 Construção a partir de um plano projetivo . . . . . . . . . . . . . . . . 21

3.7 Consideração final . . . . . . . . . . . . . . . . . . . . . . 25

4 O grafo de Kř́ǐz $\quad 27$

4.1 A construção do grafo de Kříž . . . . . . . . . . . . . . . . . . . . 27

4.2 O número de vértices do grafo de Kř́ǔz $\ldots \ldots \ldots$. . . . . . . . . . . . . . . . 32

$\begin{array}{llll}5 & \text { O } & \text { Amálgama } & 37\end{array}$

5.1 A amalgamação . . . . . . . . . . . . . . . . . . . 37

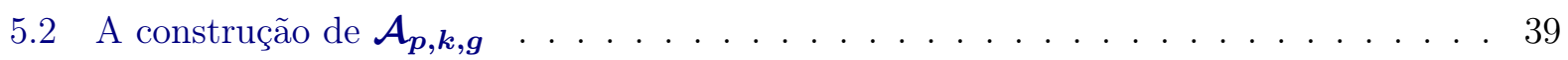

5.3 O número de vértices de $\mathcal{A}_{p, k, g} \ldots \ldots \ldots \ldots \ldots \ldots \ldots$

$6 \quad$ As árvores aumentadas $\quad 53$

6.1 A construção dos $(\boldsymbol{d}, \boldsymbol{r}, \boldsymbol{g})$-grafos $\ldots \ldots \ldots \ldots \ldots \ldots$

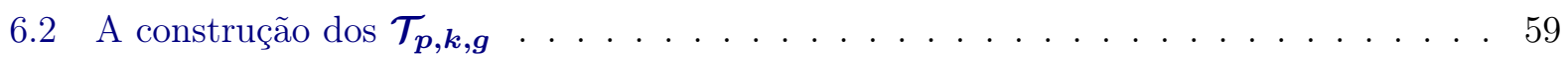

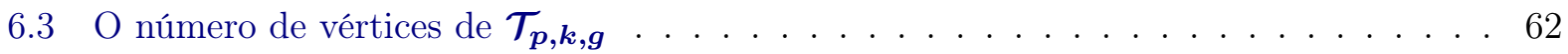

6.3 .1 Uma cota superior para $\boldsymbol{m}(\boldsymbol{d}, \boldsymbol{r}, \boldsymbol{g}) \ldots \ldots \ldots \ldots . \ldots \ldots \ldots$

6.3 .2 Uma cota inferior para $\boldsymbol{m}(\boldsymbol{d}, \boldsymbol{r}, \boldsymbol{g}) \ldots \ldots \ldots 6$

$\begin{array}{lll}7 & \text { Considerações finais } & 87\end{array}$

$\begin{array}{lr}\text { Bibliografia } & 89\end{array}$ 


\section{Lista de Figuras}

1.1 Um hipergrafo 3-uniforme contendo um circuito de comprimento 2 e outro de com-

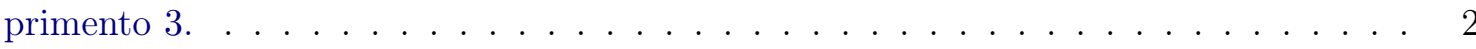

3.1 Construção de Tutte. . . . . . . . . . . . . . . . . . . . . . . . . 11

3.2 Os grafos de Tutte tem cintura pelo menos $6 \ldots \ldots \ldots \ldots \ldots \ldots$

3.3 Construção de Zykov. . . . . . . . . . . . . . . . . . . . . . . . . . . . . 13

3.4 Construção de Mycielski. Tem-se que $M_{3}$ é $C_{5}$ e que $M_{4}$ é o grafo de Grötzsch. . . . 14

3.5 O grafo $S_{5}$ tem número cromático $3 \ldots \ldots \ldots \ldots \ldots \ldots$

3.6 Os vértices são coloridos segundo seu menor elemento. . . . . . . . . . . . . . . . 17

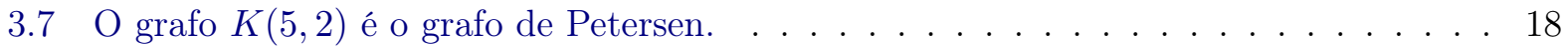

3.8 O ponto $x$ pertence $O_{i}$ quando o hemisfério $H_{x}$ contém um vértice $v \in V_{i} \ldots \ldots$

3.9 Existem vértices disjuntos contidos numa mesma parte. . . . . . . . . . . . . . 19

3.10 O plano de Fano. . . . . . . . . . . . . . . . . . . . . . . . . . . 22

3.11 Um circuito não orientado em $H$ formaria um circuito de comprimento 4 no grafo de incidência. . . . . . . . . . . . . . . . . . . . . 22

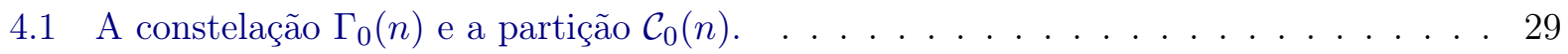

4.2 O grafo $\Gamma_{i+1}(n+1)$. As partes de $\mathcal{C}_{i+1}(n+1)$ são os retângulos maiores. $\quad \ldots \ldots$. . 30

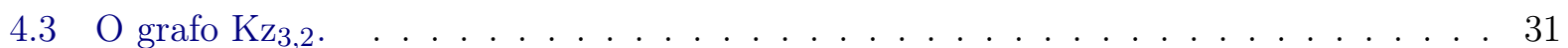

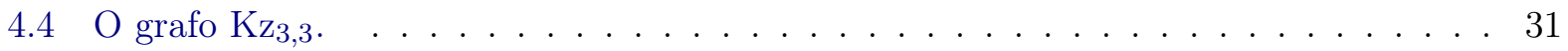

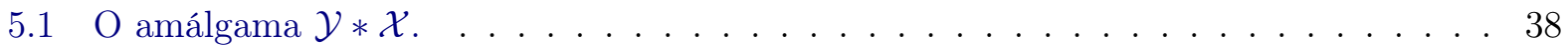

5.2 Construção da sequência $\left(\mathcal{X}^{1}, \ldots, \mathcal{X}^{4}\right) \ldots \ldots \ldots \ldots \ldots \ldots$

5.3 Um circuito em $\mathcal{X}^{t+1}$ induz um circuito em $\mathcal{A}_{\left|X_{t}^{t}\right|, k, g-1} \ldots \ldots \ldots \ldots \ldots \ldots$

5.4 Círculos iguais correspondem a arestas iguais de $\mathcal{A}_{\left|X_{t}^{t}\right|, k, g-1}$. Em cima se tem $\boldsymbol{N}$, embaixo a subsequência. . . . . . . . . . . . . . . . . . . . . 41

5.5 Esquema da construção de $\mathcal{A}_{2,3, g}$. Uma 2-coloração de $\mathcal{A}_{2,3, g}$ gera arestas monocromáticas em cada $\mathcal{A}_{\left|X_{t}^{t}\right|, 3, g-1}$. Está destacada a cópia de $\mathcal{X}^{1}$ em que cada parte é

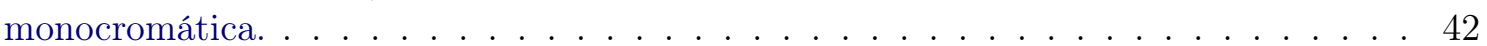

5.6 Estão marcadas as arestas monocromáticas $N_{t}(t \in[3])$, segundo a coloração induzida pelas cópias circuladas de cada $\mathcal{A}_{\left|X_{t}^{t}\right|, 3, g-1}$. Em $\mathcal{A}_{2,3, g}$, estão marcados os conjuntos monocromáticos $Z_{t} \ldots \ldots \ldots \ldots \ldots \ldots \ldots \ldots$

5.7 Em cada $\mathcal{X}^{t+1}(t \in[3])$, estão marcadas as cópia dos $\mathcal{X}^{t^{\prime}}\left(t^{\prime} \in[t]\right)$ anteriores indicadas pelas escolhas das arestas $N_{1}, \ldots, N_{t^{\prime}} \ldots \ldots \ldots \ldots 44$

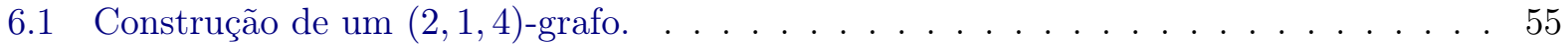


6.2 Construção de um $(d, 1, g+1)$-grafo a partir de um $(d, d, g)$-grafo. . . . . . . . . 55

6.3 Construção do subgrafo $H$ a partir de um $\left(d^{m_{1}}, r, g+1\right)$-grafo. . . . . . . . . . . 56

6.4 Construção de um $(d, r+1, g+1)$-grafo a partir de $H \subset G_{2}$ e cópias de $G_{1} \ldots \ldots 7$

6.5 Contraindo as cópias de $G_{1}$ de volta às estrelas e o circuito em $G_{2}$. . . . . . . . . 58

6.6 Um circuito de $\mathcal{T}_{p, k, g}$ corresponde a um circuito em $G$. . . . . . . . . . . . . . . 59

6.7 A folha $v_{0}$ está associada à mesma sequência tanto para a enumeração das arestas, quanto para a coloração dos vértices. . . . . . . . . . . . . . . . . 60

6.8 Uma representação para o caso $p=2, k=3, g=2$, em que $G$ é um $(2,3,5)$-grafo e $\phi$ é uma 2-coloração das arestas. Mostra-se como obter a aresta $e_{v}$ de $\mathcal{T}_{2,3,5}$. . . . 61

6.9 As folhas da subárvore atingem ancestrais distintos no caminho entre $x$ e a raiz. . . . 67

6.10 O grafo $G$ é um $T B(x, r, g)$-grafo. . . . . . . . . . . . . . . . 68

6.11 Tome $\ell \in \mathbb{L}(x, r, g)$. Para cada $G \in T B(x, r, g)$, existem $\ell^{\prime} \geq \ell$ e um conjunto $A_{G, \ell^{\prime}} \subset$

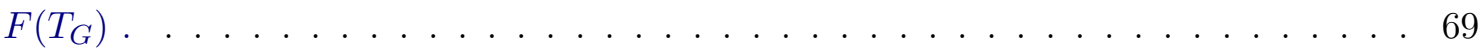

6.12 Para este $T B(x, 1, g)$-grafo $H$ não há como tomar $\ell^{\prime}$ válido. . . . . . . . . . . . 70

6.13 Quando $3 \geq g \geq 1$ e $r<x+1$, não se define $\ell(x, r, g) \ldots \ldots \ldots \ldots$

6.14 Para encontrar $\ell^{\prime}$ válido, particione os níveis e mostre que um conjunto grande das folhas não repete intervalo. . . . . . . . . . . . . . . . . . . . . . 72

6.15 Há mais folhas em $F\left(T_{G}^{\prime}\right) \cap A_{i_{0}}$ do que pares de ancestrais a serem atingidos em $I_{i_{0}} . \quad 73$

6.16 O grafo auxiliar $F$ estará contido em $G \ldots \ldots \ldots \ldots$. . . . . . . . . 75

6.17 Tomando $\ell_{0} \geq \ell\left(x_{0}, r_{0}, 2^{s}\right)$, o tamanho de $A_{G}$ não é grande o suficiente. . . . . . 76

6.18 Reservam-se 3 arestas para garantir que níveis mais altos sejam atingidos e, assim, obter que $A_{G}^{\prime \prime}$ é grande suficiente. . . . . . . . . . . . . . . . . . 76

6.19 Primeiro, define-se o grafo auxiliar $F^{\prime} \ldots \ldots \ldots \ldots \ldots \ldots$

6.20 Devolvendo as arestas extras a $A_{F^{\prime \prime}, \ell^{\prime \prime}}$, encontra-se que boa parte de $F\left(T_{F}\right)$ envia arestas acima de um nível mais alto $\ell^{\prime}>\ell^{\prime \prime} \ldots \ldots \ldots$. . . . . . . . . . 80

6.21 Deixe apenas as duas arestas mais baixas de cada folha para definir $F^{\prime} \subset G$. . . . 81

6.22 Encontra-se $F \in T B\left(\ell\left(x, 2,2^{s+1}\right), r-1,2^{s+1}\right)$ em $G$. . . . . . . . . . . . . . 82

6.23 Obtenha $F \in T B(100,2 r, g / 2-1)$ a partir de um $(2,1, g)$-grafo $G$. . . . . . . . 84

6.24 Um circuito de tamanho $2 k$ em $F$ induz um circuito de tamanho no máximo $4 k$ em $G$. 85 


\section{Capítulo 1}

\section{Introdução}

A cintura e o número cromático de um grafo são dois parâmetros que podem ser utilizados para se analisar a distribuição e a densidade de suas arestas. Assim, é interessante entender como eles se relacionam.

Definição 1.0.1. Seja $G$ um grafo. A cintura de $G$, denotada por $\operatorname{cin}(G)$, é o menor comprimento de um circuito de $G$. O número cromático de $G$, denotado por $\chi(G)$, é o menor inteiro $k$ tal que existe uma coloração dos vértices de $G$ com $k$ cores, sem que haja aresta com ambos extremos da mesma cor.

Um grafo com cintura grande é "esburacado", enquanto um grafo com número cromático grande é cheio de conexões. Mas se fossem parâmetros realmente antagônicos, não existiriam grafos com cintura e número cromático grandes. A cintura grande garante uma esparsidade apenas local, que não impede conexões intrincadas que aumentam o número cromático.

Inicialmente, eram conhecidas apenas construções de grafos livres de triângulo e com número cromático alto. Foi probabilisticamente que se provou a existência de grafos e hipergrafos uniformes com cintura e número cromático grandes [12], [13].

Definição 1.0.2. Um hipergrafo p-uniforme ou p-grafo é um par $(X, \mathcal{M})$. O conjunto dos vértices $X$ é um conjunto qualquer. O conjunto das arestas $\mathcal{M}$ é tal que

$$
\mathcal{M} \subseteq\left(\begin{array}{c}
X \\
p
\end{array}\right)=\{M \subseteq X:|M|=p\}
$$

Um circuito de comprimento $\ell \geq 2$ em um hipergrafo é uma sequência de arestas distintas, $\left(e_{1}, e_{2}, \ldots, e_{\ell}\right)$, tal que para todo $i \in[\ell]=\{1, \ldots, \ell\}$, existe $x_{i} \in e_{i} \cap e_{i+1}$ (onde $e_{\ell+1}=e_{1}$ ) e, para quaisquer $i \neq j \in[\ell]$, vale que $x_{i} \neq x_{j}$. (Figura 1.1 )

A cintura de um hipergrafo é o menor comprimento de um circuito nesse hipergrafo. O número cromático de um $p$-grafo $\mathcal{H}$ é o número mínimo de cores necessárias para se colorir os vértices de $\mathcal{H}$ sem que haja aresta tal que todos seus $p$ vértices tenham a mesma cor.

As demonstrações probabilísticas garantem a existência, mas não apresentam uma construção para grafos e hipergrafos com cintura e número cromático grandes. Assim, ainda não fica claro como se obter um grafo com essas características. A primeira construção foi encontrada por Lovász [24]. Essa construção envolve hipergrafos mesmo no caso em que se busca um grafo com cintura e número cromático grandes. A construção de Lovász não será abordada neste trabalho. Em seu lugar, será 


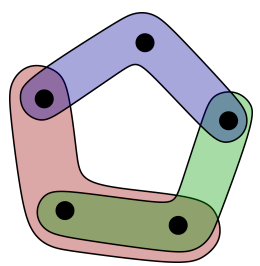

Figura 1.1: Um hipergrafo 3-uniforme contendo um circuito de comprimento 2 e outro de comprimento 3.

apresentada a construção de Nešetřil e Rödl [33]. Essa construção, seguinte à de Lovász, é mais simples, mas também envolve hipergrafos. Ela se baseia na amalgamação de hipergrafos $p$-uniformes e $a$-partidos, para certos parâmetros $a$ e $p$ que dependem da uniformidade e do número cromático desejados. A amalgamação é uma forma de combinar dois hipergrafos para se obter um terceiro. A amalgamação entre os hipergrafos $\mathcal{H}_{1}$ e $\mathcal{H}_{2}$ "costura" cópias disjuntas de $\mathcal{H}_{2}$. A forma em que as cópias são costuradas depende de $\mathcal{H}_{1}$. Essa construção é indutiva no tamanho da cintura.

No entanto, parecem existir grafos mais simples com cintura e número cromático grandes. Isso, porque é possível calcular o número de vértices do grafo dado pela demonstração probabilística para cada valor de cintura e número cromático. Esse número de vértices é bastante menor que o do grafo obtido por qualquer uma das construções conhecidas até agora para os mesmos valores de cintura e número cromático.

Possivelmente, bastaria uma construção para grafos não envolver hipergrafos para ser mais simples e ter menos vértices. A construção de Kř́už [23] foi a primeira a conseguir grafos com cintura e número cromático grandes sem utilizar hipergrafos. Nela, constrói-se primeiro uma família de grafos auxiliares. Os grafos dessa família têm dois parâmetros, que dependem da cintura e do número cromático desejados. A construção dessa família é duplamente indutiva; tem-se uma indução para cada um dos parâmetros. Nela, assim como na amalgamação, são tomadas várias cópias de um grafo já obtido por indução, sendo que o número de cópias tomadas depende de outro grafo também já obtido por indução. Porém essas cópias e esse outro grafo não serão "costurados", mas apenas unidos de forma disjunta entre si. A construção, de fato, do grafo com cintura e número cromático desejados parte de um grafo com cintura grande, mas número cromático insuficiente. Para aumentar o número cromático, diversas cópias desse grafo inicial são "inseridas" num certo grafo da família auxiliar. O grafo obtido é então inserido em outro grafo da família auxiliar até se obter o número cromático desejado. Mesmo sem envolver hipergrafos, o número de vértices do grafo obtido por essa construção para dada cintura e número cromático também é muito grande.

Tanto a construção por amalgamação quanto a de Kříž envolvem repetir certo procedimento. Essa natureza indutiva pode ser evitada. Mais recentemente, Alon, Kostochka, Reiniger, West e Zhu [4] obtiveram uma outra construção que fornece diretamente, a partir de um grafo auxiliar, um hipergrafo uniforme com cintura e número cromático grandes. Assim, ela também consegue um grafo com cintura e número cromático grandes sem envolver hipergrafos. A família de grafos auxiliares é constituída por certas árvores aumentadas que têm 3 parâmetros, que dependem da uniformidade do hipergrafo, do número cromático e da cintura desejados. A construção descrita para a família auxiliar é duplamente indutiva. Ela também envolve tomar diversas cópias de uma certa árvore aumentada dada por indução e combiná-las com outra árvore aumentada dada por indução. O número de vértices do grafo obtido por essa construção para certos valores de cintura e número cromático é também muito grande, quando comparado ao fornecido pela demonstração 
probabilística.

Se o grafo procurado deve ter cintura e número cromático maiores que um certo valor $x$, a ordem do número de vértices dessas construções não é nem linear, nem exponencial ou torre, na verdade, o número de vértices é como a função de Ackermann. A função de Ackermann é definida recursivamente e possui um parâmetro segundo o qual se muda de hierarquia, por exemplo, de multiplicação para exponenciação, que é a multiplicação feita repetidas vezes. As flechas de Knuth são uma boa maneira de representar a função de Ackermnann; um certo número de flechas corresponde a um certo nível da hierarquia de Ackermann. Um dos objetivos deste trabalho é mostrar cotas explícitas para o número de vértices de cada uma das construções. Os resultados obtidos estão resumidos no seguinte Teorema 1.0.1.

Teorema 1.0.1. Sejam $k \geq 4$ e g $>51$ inteiros. Chame respectivamente de $\mathrm{Kz}_{k, g}$, de $\mathcal{A}_{2, k, g}$ e de $\mathcal{T}_{2, k, g}$ os grafos obtidos pelas construções de Kř̌ž, por amalgamação e por árvores aumentadas com cintura maior que $g$ e número cromático pelo menos $k$. Vale que

$$
\begin{array}{ll}
2 \uparrow\left\lceil\log _{2} g\right\rceil(k+8) & \geq\left|\mathrm{Kz}_{k, g}\right| \geq 2 \uparrow\left\lceil\log _{2} g\right\rceil(k-1), \\
(3(k-1)) \uparrow^{g}(4 k+2) & \geq\left|\mathcal{A}_{2, k, g}\right| \geq\left(\frac{k-1}{2}\right) \uparrow g(k-1), \\
(k-1) \uparrow^{2 g-1}\left(6^{k}+1\right) & \left.\geq\left|\mathcal{T}_{2, k, g}\right| \geq 2 \uparrow \log _{2}(g-1)\right\rfloor 2(k-1) .
\end{array}
$$

Outro objetivo deste trabalho é descrever em detalhes essas construções de grafos e hipergrafos com cintura e número cromático grandes. As construções estão ordenadas da seguinte forma. No Capítulo 3, são apresentadas construções de grafos livres de triângulos e com número cromático grande, algumas bastante conhecidas. São apresentados os grafos de Tutte [37], Zykov [39], Mycielski [28], Kneser [19], os grafos de shift de Erdôs e Hajnal [15] e os grafos de planos projetivos finitos [11]. Para cada uma dessas construções, analisa-se seu número de vértices.

No Capítulo 4, é descrita a construção de Kř̌iž para grafos de cintura e número cromático grandes, que não envolve hipergrafos. Primeiro se constrói a família de grafos auxiliares. Utilizando os grafos dessa família, constroem-se os grafos de Kř́žz. Em seguida, calculam-se uma cota superior e uma inferior para seu número de vértices.

No Capítulo 5, é descrita a construção de Nešetřil e Rödl, que utiliza a amalgamação de hipergrafos. Primeiro se define a amalgamação. Depois é descrita a construção dos hipergrafos com cintura e número cromático grandes. Por último, são calculadas uma cota inferior e uma superior para o número de vértices do hipergrafo obtido.

No Capítulo 6, apresenta-se a construção de Alon, Kostochka, Reiniger, West e Zhu que utiliza árvores aumentadas. Primeiro certas árvores aumentadas são construídas. Depois elas são utilizadas para a construção de hipergrafos com cintura e número cromático grandes. Segue uma cota superior para número de vértices dessa construção. Para se obter uma cota inferior, são definidos outros grafos auxiliares. Eles são parecidos com árvores aumentadas; seus vértices também se baseam nos vértices uma árvore. A altura mínima da árvore de um desses grafos está relacionada à altura mínima de uma árvore aumentada. O número de vértices do hipergrafo com cintura e número cromático grandes obtido a partir de certa árvore aumentada pode ser determinado a partir do número de vértices dessa árvore aumentada. Assim, calcula-se uma cota para a altura mínima das árvores desses outros grafos auxiliares.

Vale uma última observação sobre grafos de cintura e número cromático grandes. Esses grafos estão relacionados com grafos expansores, que são relevantes para as teorias de algoritmos e de complexidade. Existem diferentes parâmetros utilizados para se caracterizar um grafo expansor. Num 
sentido geral, são grafos esparsos, mas altamente conexos. Lubotzky, Phillips e Sarnak [26] encontraram uma construção com número polinomial de vértices para grafos expansores. Essa construção é resultado de uma combinação de teoria dos números com análise harmônica e teoria algébrica dos grafos. Os grafos obtidos são certos grafos de Cayley. Eles são grafos regulares e têm máxima conectividade segundo certo parâmetro. Grafos regulares que são expansores ótimos são chamados de grafos de Ramanujan. Além disso, prova-se que esses grafos têm cintura e número cromático grandes. Essa construção não é elementar no sentido em que é preciso determinar um certo grupo e um certo conjunto de geradores desse grupo para se obter o grafo de Cayley desejado, em vez de se ter uma descrição estrutural da construção. Este trabalho não apresenta essa construção; grafos expansores é um assunto bastante vasto e um leitor interessado pode consultar [18] para saber mais sobre eles. Por fim, observa-se que ainda não é conhecida uma generalização dessa construção para hipergrafos. De fato, não se tem nenhuma construção explícita de hipergrafos com cintura e número cromático grandes com poucos vértices. 


\section{Capítulo 2}

\section{Considerações iniciais}

Um fato muito conhecido é que proibir circuitos de comprimento ímpar num grafo garante que seu número cromático é no máximo 2. Circuitos ímpares são subgrafos que não têm um tamanho fixo; podem ter muitos vértices. Pode ser questionado se, para algum inteiro positivo $g$, existiria alguma estrutura com até $g$ vértices cuja proibição restringisse o número cromático de $G$. Ou ainda, sendo $n$ o número de vértices de $G$, se proibir alguma estrutura com até $g(n)$ vértices poderia garantir número cromático pequeno.

Um grafo $H$ com até $g$ vértices que não seja uma árvore tem certamente $\operatorname{cin}(H) \leq g$. Se um grafo não contém circuitos, ele é uma árvore e sua cintura é dita infinita. Então, se $\operatorname{cin}(G)>g$, o grafo $H$ não pode estar contido em $G$. Dessa forma, proibir todos subgrafos que não sejam árvores e que tenham até certo tamanho pode ser convertido em analisar grafos de cintura grande. Com relação a proibir uma dada árvore $T$ de até $g$ vértices, pode-se mostrar que isso limita o número cromático.

Proposição 2.0.1. Se $T$ é uma árvore com g vértices e $T \not \subset G$, então $\chi(G) \leq g-1$.

Ideia da demonstração. Seja $n$ o número de vértices de $G$. Faça uma indução em $n$. Quando $n=g$, tem-se que $G \neq K_{g}$, senão conteria $T$. Então $G \subseteq K_{g}-\{u, v\}$, onde $\{u, v\}$ é uma aresta de $K_{g}$. Tome uma coloração $c$ dos vértices de $G$ com cores em $[g-1]$ tal que $c(u)=c(v)$ e que, para cada um dos demais vértices, é dada uma cor distinta; ela é própria. Para $G$ com $n+1$ vértices, considere um subgrafo maximal $T^{\prime} \subset T$ que esteja em $G$. Será encontrado um vértice de grau pequeno em $G$. O grau de um vértice $v$ em $G$ será denotado $d_{G}(v)$. Deve existir um vértice $v$ em $T^{\prime}$ tal que $d_{T}(v)>d_{T^{\prime}}(v)$. Como $T^{\prime}$ é subgrafo maximal, todos vizinhos de $v$ em $G$ devem estar contidos em $T^{\prime}$. Dessa forma, $d_{G}(v) \leq g-2$. Removendo $v$, aplica-se a hipótese de indução para conseguir uma coloração com $g-1$ cores. Alguma dessas cores poderá ser atribuída a $v$, pois ele possui no máximo $g-2$ vizinhos.

Se um grafo tem cintura maior que $g$, então ele se parece localmente com uma árvore, pois a partir de qualquer vértice, num raio de $g / 2-1$ arestas, não há circuito. Uma árvore tem número cromático 2. Seria possível um grafo com cintura grande ter número cromático grande também? Essa pergunta já foi bastante estudada e exemplos de grafos com cintura e número cromático grandes aparecem em diversos contextos em Combinatória. Foi uma pergunta que serviu e ainda serve como motivação para o desenvolvimento da área. Por isso, Nešetřil [31] considera o problema clássico.

Define-se a família de hipergrafos que será alvo do estudo. 
Definição 2.0.1. Dados $p \geq 2, k \geq 1$ e $g \geq 2$ inteiros, denote por $\mathcal{G}_{p, k, g}$ o conjunto de $p$-grafos tal que $\mathcal{H} \in \mathcal{G}_{p, k, g}$ se e somente se

$$
\begin{aligned}
\operatorname{cin}(\mathcal{H}) & >g \mathrm{e} \\
\chi(\mathcal{H}) & \geq k .
\end{aligned}
$$

Quando $p=2$, às vezes será omitido o índice $p$; usa-se que $\mathcal{G}_{2, k, g}=\mathcal{G}_{k, g}$.

\subsection{Demonstrações probabilísticas}

A primeira demonstração de que existiam grafos em $\mathcal{G}_{k, g}$, para quaisquer $k \geq 1$ e $g \geq 2$, foi feita por Erdôs, utilizando o método probabilístico, num momento em que estudava números de Ramsey [12].

Para $p$ e $q$ inteiros positivos, o número de Ramsey $R(p, q)$ é o menor inteiro tal que qualquer grafo com $R(p, q)$ vértices conterá um grafo completo com $p$ vértices ou um conjunto independente de tamanho $q$. Assim, se $R(3, q)>n$, com $n=n(q)$, então existe algum grafo $G$ com $n$ vértices que é livre de triângulos e que não tem nenhum conjunto independente de tamanho q. O número de independência, denotado por $\alpha(G)$, é o maior tamanho de um conjunto independente de $G$. Já que, numa coloração dos vértices, cada cor define um conjunto independente, tem-se a relação

$$
\chi(G) \geq \frac{n}{\alpha(G)} .
$$

Ela permite concluir que, para o grafo $G$ mencionado acima, vale que $\chi(G) \geq n / q$. Assim, se valer que $R(3, q)>n(q)$ e $\lim _{q \rightarrow \infty} n(q) / q \rightarrow \infty$, prova-se a existência de grafos livres de triângulos e com número cromático tão grande quanto se queira.

Para os casos em que $g>3$, não apenas o grafo completo de 3 vértices é proibido, portanto é preciso definir um outro número, semelhante ao número de Ramsey. O valor $h(g, q)$ é o menor inteiro tal que qualquer grafo com $h(g, q)$ vértices ou contém algum circuito de comprimento menor que ou igual a $g$ ou contém um conjunto independente de tamanho $q$. Foi nesse contexto que se demonstrou, pela primeira vez, o Teorema 2.1.1 que, aqui, é enunciado como em [8]. No Teorema 2.1.1 e no decorrer do texto, dado um grafo $G$, o seu número de vértices é denotado por $|G|$.

Teorema 2.1.1. Dados inteiros $k \geq 2$ e $g \geq 3$, existe um grafo $G \in \mathcal{G}_{k, g-1}$ e $|G|=k^{3 g}$.

Ideia da Demonstração. A demonstração deste teorema considera o espaço de probabilidade $\mathcal{G}(n, p)$ dos grafos com $n$ vértices tais que cada aresta ocorre independentemente com probabilidade $p$. Assim, um grafo com $m$ arestas tem probabilidade $p^{m}(1-p)^{\left(\begin{array}{c}n \\ 2\end{array}\right)-m}$. Toma-se $n=k^{3 g}$ e $p=2 k^{2} / n$.

Para $a=2^{g-1} k^{2 g-2}$, calcula-se a probabilidade de um grafo ter até $a$ circuitos de comprimento menor que ou igual a $g-1$. Esse valor deve ser maior que $1 / 2$. Depois, calcula-se a probabilidade de todo subconjunto dos vértices de tamanho $s=n / k$ induzir pelo menos $a+1$ arestas. Essa probabilidade também deve ser maior que $1 / 2$.

Assim, a probabilidade da intersecção será maior que 0 e, portanto, existirá algum grafo $G^{\prime}$ com até $a$ circuitos de comprimento no máximo $g-1$ e tal que todo subconjunto de tamanho $s$ induz pelo menos $a+1$ arestas. Apagando uma aresta de cada circuito pequeno, no máximo $a$ arestas serão removidas, de forma que todo subconjunto de tamanho $s$ ainda deve induzir pelo 
menos uma aresta. Consegue-se um outro grafo $G$, com $\operatorname{cin}(G)>g-1$ e $\alpha(G)<s$. Portanto, tem-se $\chi(G)>n / s=k$.

Existe outra forma de utilizar o método probabilístico e provar que existe um grafo em $\mathcal{G}_{k, g}$, que pode ser lida em [5]. Nessa outra demonstração, procura-se um grafo $G^{\prime}$ que deve ter duas propriedades. A primeira é que $G^{\prime}$ tem até $\left|G^{\prime}\right| / 2$ circuitos de comprimento no máximo $g$. A segunda é que $\alpha\left(G^{\prime}\right)$ é no máximo uma certa função $f\left(\left|G^{\prime}\right|\right)$. Escolhe-se certo valor para $p$ e prova-se que a probabilidade de $G^{\prime}$ é positiva, de modo que ele é encontrado. A partir de $G^{\prime}$, constrói-se $G$ removendo um vértice de cada circuito pequeno. Tem-se $\alpha(G) \leq \alpha\left(G^{\prime}\right) \leq f\left(\left|G^{\prime}\right|\right)$. Assim, vale que $\chi(G) \geq|G| / \alpha(G) \geq\left|G^{\prime}\right| /\left(2 f\left(\left|G^{\prime}\right|\right)\right)$. Por fim, prova-se que para $\left|G^{\prime}\right|$ suficientemente grande, tem-se $\chi(G) \geq\left|G^{\prime}\right| /\left(2 f\left(\left|G^{\prime}\right|\right)\right) \geq k$.

As demonstrações probabilísticas garantem a existência de grafos em $\mathcal{G}_{k, g}$, respondendo negativamente à questão de se existiria algum subgrafo com um número limitado de vértices cuja proibição limita superiormente o número cromático. Porém essas demonstrações não apresentam uma construção para tais grafos em $\mathcal{G}_{k, g}$. A primeira construção encontrada precisou utilizar hipergrafos e, na verdade, construiu para qualquer $p \geq 2$, um $p$-grafo com cintura e número cromático grandes.

Antes dessa construção, a existência de hipergrafos uniformes com cintura e número cromático grandes também já havia sido garantida por Erdốs e Hajnal [13], utilizando o método probabilístico.

Teorema 2.1.2. Para $p \geq 2, k \geq 1$ e $g \geq 2$ inteiros, existe um p-grafo em $\mathcal{G}_{p, k, g}$.

Ideia da Demonstração. Nešetřil e Rödl apresentam uma demonstração para esse teorema em [29]. Primeiro, prova-se que para todos $p \geq 3, \ell \geq 3$ e $\varepsilon>0$ existe um inteiro $n_{0}(p, \ell, \varepsilon)$ tal que para todo $n \geq n_{0}$, existe um $p$-grafo $\mathcal{H}(n, p, \ell, \varepsilon)=(X, \mathcal{M})$ com as seguintes características.

1. $|X|=n$;

2. $|\mathcal{M}|=\left\lceil n^{1+\varepsilon}\right\rceil ;$

3. $\operatorname{cin}(\mathcal{H}) \geq \ell$.

Um hipergrafo $\mathcal{H}(n, p, \ell, \varepsilon)$ é obtido sorteando-se uniformemente um hipergrafo $\mathcal{H}$ dentre todos $p$ grafos com $n$ vértices e $2\left\lceil n^{1+\varepsilon}\right\rceil$ arestas. Calcula-se a esperança do número de circuitos menores que $\ell$, que é $o(n)$. Assim, a probabilidade de $\mathcal{H}$ ter mais que $\left\lceil n^{1+\varepsilon}\right\rceil$ circuitos menores que $\ell$ tende a 0 , quando $n \rightarrow \infty$. Para $n$ grande, existe $\mathcal{H}$ com no máximo $\left\lceil n^{1+\varepsilon}\right\rceil$ circuitos pequenos. Apagando uma aresta para cada circuito, obtém-se $\mathcal{H}(n, p, \ell, \varepsilon)$.

Agora tome $\mathcal{H}(n,(p-1)(k-1)+1, g+1, \varepsilon)=\left(X_{0}, \mathcal{M}_{0}\right)$. O $p$-grafo em $\mathcal{G}_{p, k, g}$ que será encontrado terá cada uma de suas arestas contida em uma aresta distinta de $\mathcal{M}_{0}$. Dessa forma, sua cintura também será pelo menos $g+1$. Mais especificamente, busca-se um grafo em $\mathcal{G}_{p, k, g}$ dentro do conjunto

$$
\mathbb{G}=\left\{\mathcal{G}=\left(X_{0}, \mathcal{N}\right): \mathcal{G} \text { é } p \text {-grafo e }\left|\mathcal{N} \cap\left(\begin{array}{c}
M \\
p
\end{array}\right)\right|=1 \text { para todo } M \in \mathcal{M}_{0}\right\} .
$$

O tamanho desse conjunto é

$$
|\mathbb{G}|=a^{\left\lceil n^{1+\varepsilon}\right\rceil}, \text { onde } a=\left(\begin{array}{c}
(p-1)(k-1)+1 \\
p
\end{array}\right)
$$


pois uma escolha de $p$ vértices para uma dada aresta de $\mathcal{M}_{0}$ não impossibilita nenhuma escolha de $p$ vértices em outra aresta. Se um mesmo conjunto de $p$ vértices pudesse ser escolhido a partir de duas arestas distintas de $\mathcal{M}_{0}$, haveria um circuito de comprimento 2 em $\mathcal{H}(n,(p-1)(k-1)+1, g+1, \varepsilon)$.

Para verificar que existe $\mathcal{G} \in \mathbb{G}$ com número cromático pelo menos $k$, veja que para toda coloração de $X_{0}$ com até $k-1$ cores, cada aresta de $\mathcal{M}_{0}$ terá pelo menos $p$ vértices da mesma cor. Portanto, o número de $p$-grafos em $\mathbb{G}$ para os quais uma dessa colorações é própria é no máximo $(a-1)^{\left\lceil n^{1+\varepsilon}\right\rceil}$. O número de colorações com até $k-1$ cores é $(k-1)^{n}$. Dessa forma, no máximo $(k-1)^{n} \cdot(a-1)^{\left\lceil n^{1+\varepsilon}\right\rceil}$ hipergrafos em $\mathbb{G}$ têm alguma coloração própria com até $k-1$ cores. Para $n$ grande, $|\mathbb{G}|=a^{\left\lceil n^{1+\varepsilon}\right\rceil}>(k-1)^{n} \cdot(a-1)^{\left\lceil n^{1+\varepsilon}\right\rceil}$. Assim, existe $p$-grafo $\mathcal{G} \in \mathbb{G}$ que não pode ser colorido com $k-1$ cores. Dessa forma, $\mathcal{G} \in \mathcal{G}_{p, k, g}$.

\subsection{Contando o número de vértices}

Nos próximos capítulos, serão descritas construções de grafos e hipergrafos com cintura e número cromático grandes. Ainda não se conhece uma construção de um grafo em $\mathcal{G}_{k, g}$ que seja elementar e tenha número de vértices da ordem de $k^{3 g}$, como dado pelo Teorema 2.1.1. Para o caso geral, não se conhece nenhuma construção de um hipergrafo em $\mathcal{G}_{p, k, g}$ com poucos vértices. Quando se quer cintura e número cromático maiores que $x$, os números de vértices dessas construções em relação a $x$ são como a função de Ackermann em certo sentido, que será descrito a seguir. Define-se a função de Ackermann [1].

Definição 2.2.1. Sejam $a \geq 0, b \geq 0$ e $n \geq 0$ inteiros. A função de Ackermann $\varphi(a, b, n)$ é dada pelas seguintes propriedades,

$$
\varphi(a, b, n)= \begin{cases}a+b, & \text { para } n=0 \\ 0, & n=1 \text { e } b=0 \\ 1, & n=2 \text { e } b=0 \\ a, & n>2 \text { e } b=0 \\ \varphi(a, \varphi(a, b-1, n), n-1), & n>0 \text { e } b>0 .\end{cases}
$$

Quando $n=0$, a função de Ackermann é a soma, $\varphi(a, b, 0)=a+b$. Quando $n=1$, é a multiplicação, $\varphi(a, b, 1)=a b$, que é uma repetição de somas. Para $n=2$, tem-se $\varphi(a, b, 2)=a^{b}$, que é uma repetição de multiplicações. Para $n=3$, tem-se

$$
\varphi(a, b, 3)=\varphi(a, \varphi(a, b-1,3), 2)=a^{\varphi(a, b-1,3)}=\underbrace{a \cdot{ }^{a}}_{b+1} .
$$

Para um certo valor de $n$, a função de Ackermann com parâmetro $n$ é a função de Ackermann com parâmetro $n-1$ aplicada repetidas vezes. A notação de flechas de Knuth [20] é uma forma conveniente de se denotar a função correspondente a $n$, para todo $n$. 
Definição 2.2.2. Para inteiros $a, b$ e $n$, com $b, n \geq 0$, tem-se

$$
a \uparrow^{n} b= \begin{cases}a b, & n=0 \\ 1, & n \geq 1 \text { e } b=0 \\ a \uparrow^{n-1}\left(a \uparrow^{n}(b-1)\right), & n \geq 1 \text { e } b \geq 1 .\end{cases}
$$

Quando $n=0$ a operação $\uparrow^{0}$ é a multiplicação. Para $n=1$, tem-se a exponenciação. Para $n \geq 2$, assim como a exponenciação $a^{b}$ é a multiplicação de uma quantidade $b$ de fatores iguais a $a$, a operação $a \uparrow^{n} b$ é a operação $\uparrow^{n-1}$ aplicada a uma quantidade $b$ de fatores iguais a $a$,

$$
a \uparrow^{n} b=\underbrace{a \uparrow^{n-1}\left(a \uparrow^{n-1}\left(\ldots \uparrow^{n-1} a\right) \ldots\right)}_{\# a=b} .
$$

Quando descrita utilizando-se as flechas de Knuth, a função de Ackermann com parâmetro $n \geq 1$ terá forma $x \uparrow^{n-1} y$, para certos $x$ e $y$ que dependem de $a, b$ e $n$. Existem outras funções que também são chamadas de função de Ackermann. Isso, porque essas funções também têm um parâmetro $m$ segundo o qual podem ser representadas por algo da forma $x^{\prime} \uparrow^{m} y^{\prime}$.

Define-se a função de Ackermann em 2 parâmetros de Péter [34] e Robinson [35].

Definição 2.2.3. A função de Ackermann em 2 parâmetros $A(m, n)$ é descrita da seguinte forma.

$$
A(m, n)= \begin{cases}n+1, & m=0 \\ A(m-1,1), & m>0 \text { e } n=0 \\ A(m-1, A(m, n-1)), & m>0 \text { e } n>0 .\end{cases}
$$

Será provado que essa função passa da soma para a multiplicação e assim por diante, segundo o parâmetro $m$.

Proposição 2.2.1. Vale que $A(1, n)=n+2$ e, para $m \geq 2$, tem-se $A(m, n)=2 \uparrow m-2(n+3)-3$.

Demonstração. Para mostrar que $A(1, n)=n+2$, faça uma indução em $n$. Tem-se

$$
A(1,0)=A(0,1)=2 .
$$

Seja $n \geq 0$, suponha que $A(1, n)=n+2$. Calcule

$$
A(1, n+1)=A(0, A(1, n))=A(1, n)+1=n+3 .
$$

Para $m \geq 2$, quer-se provar $A(m, n)=2 \uparrow^{m-2}(n+3)-3$. Faça uma indução em $m$. No caso base, para qualquer $n \geq 0$, é preciso que $A(2, n)=2(n+3)-3=2 n+3$. Faça uma indução em $n$. Tem-se

$$
A(2,0)=A(1,1)=A(0, A(1,0))=A(0,2)=3 .
$$

Seja $n \geq 0$ e suponha que $A(2, n)=2 n+3$. Tem-se

$$
A(2, n+1)=A(1, A(2, n))=A(2, n)+2=2(n+1)+3 .
$$


Para o passo da indução em $m$, seja $m \geq 2$ e suponha que $A(m, n)=2 \uparrow^{m-2}(n+3)-3$ para todo $n \geq 0$. Para calcular $A(m+1, n)$, faça uma indução em $n$. Tem-se

$$
\begin{aligned}
A(m+1,0) & =A(m, 1)=2 \uparrow^{m-2} 4-3=2 \uparrow^{m-2}(2 \uparrow 2)-3 \\
& =2 \uparrow^{m-2}\left(2 \uparrow^{m-1} 2\right)-3=2 \uparrow^{m-1} 3-3 .
\end{aligned}
$$

Seja $n \geq 0$ e suponha que $A(m+1, n)=2 \uparrow^{m-1}(n+3)-3$. Tem-se

$$
\begin{aligned}
A(m+1, n+1) & =A(m, A(m+1, n))=A\left(m, 2 \uparrow^{m-1}(n+3)-3\right) \\
& =2 \uparrow^{m-2}\left(2 \uparrow^{m-1}(n+3)-3+3\right)-3=2 \uparrow^{m-1}(n+4)-3,
\end{aligned}
$$

completando a demonstração.

Os números de vértices das construções que serão vistas serão Ackermannianos no seguinte sentido. Para cada uma delas, se o hipergrafo obtido tem cintura e número cromático maiores que $x$, então tanto a cota superior quanto a inferior para o número de vértices são funções com um número de flechas de Knuth que depende de $x$. 


\section{Capítulo 3}

\section{Grafos sem triângulos com número cromático grande}

As primeiras construções consideravam a versão do problema para grafos e o menor caso não trivial da cintura, $g=3$. Assim, buscava-se uma construção que, para qualquer $k$ inteiro positivo, fornecesse um grafo livre de triângulos e com número cromático pelo menos $k$. Neste capítulo, são apresentadas diversas construções para esses grafos.

\subsection{O grafo de Tutte}

A construção de Tutte [37] para $T_{k} \in \mathcal{G}_{k, 3}$ é indutiva em $k$ e, na realidade, os grafos construídos são tais que $\operatorname{cin}\left(T_{k}\right) \geq 6$. Para $k=1$, tome $T_{1}$ o grafo constituído de um único vértice. Tendo construído $T_{k}$, para construir $T_{k+1}$, tome um conjunto independente $A$ de tamanho $k\left(\left|T_{k}\right|-1\right)+1$. Agora, para cada subconjunto $A_{i} \subset A$ com $\left|A_{i}\right|=\left|T_{k}\right|$, tome uma cópia $T_{k}^{i}$ de $T_{k}$ e faça um emparelhamento perfeito entre os vértices dessa cópia e os vértices de $A_{i}$. Esse grafo será $T_{k+1}$ (Figura 3.1).

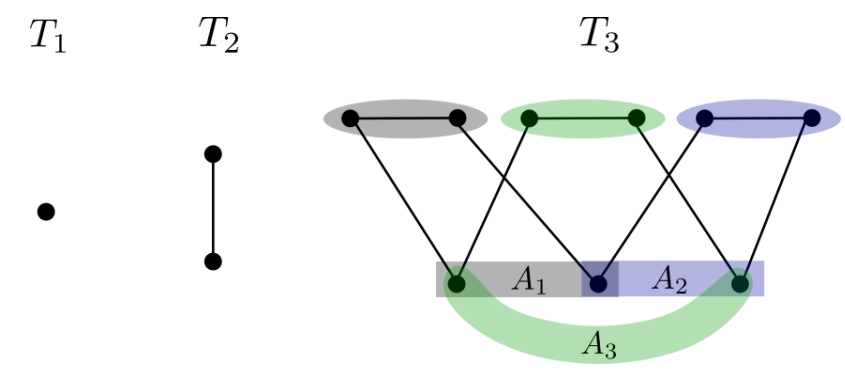

Figura 3.1: Construção de Tutte.

O número cromático $\chi\left(T_{k+1}\right)$ é igual a $k+1$. De fato, $k+1$ cores são suficientes para uma coloração própria dos vértices de $T_{k+1}$, pois cada $T_{k}^{i}$ pode ser colorido propriamente com as cores em $[k]$ e, como $A$ é independente, ele pode ser todo colorido com a cor $k+1$. Para verificar que $\chi\left(T_{k+1}\right) \geq k+1$, tome uma coloração arbitrária com $k$ cores e mostre que existe uma aresta monocromática. Como $|A|=k\left(\left|T_{k}\right|-1\right)+1$, pelo princípio da casa dos pombos, uma das cores foi atribuída a pelo menos $\left|T_{k}\right|$ vértices de $A$. Seja $A_{i_{0}}$ esse conjunto monocromático de tamanho $\left|T_{k}\right|$. Ao conjunto $A_{i_{0}}$, está associada a cópia $T_{k}^{i_{0}}$. Se a coloração de $T_{k}^{i_{0}}$ é própria, então as $k$ cores foram utilizadas por hipótese de indução. Mas se algum vértice de $T_{k}^{i_{0}}$ utiliza a cor de $A_{i_{0}}$, então alguma 
aresta do emparelhamento terá ambos extremos da mesma cor. Portanto, o número cromático do grafo construído é pelo menos $k+1$.

Agora, considere um circuito desse grafo. Se ele estiver contido numa cópia de $T_{k}$, sabe-se por indução que seu comprimento é pelo menos 6 . Senão, ele deve conter um vértice $v_{1} \in A$. Desse vértice, saem duas arestas do circuito $v_{1} x_{1}$ e $v_{1} x_{2}$. Como as arestas que incidem em $A$ são arestas de algum emparelhamento perfeito entre certo $A_{i}$ e uma cópia $T_{k}^{i}$, então $x_{1}$ e $x_{2}$ pertencem a cópias distintas $T_{k}^{i_{1}}$ e $T_{k}^{i_{2}}$ e $v_{1} \in A_{i_{1}} \cap A_{i_{2}}$. Como as cópias de $T_{k}$ são disjuntas, é preciso que o circuito contenha pelo menos outro vértice $v_{2} \in A$. Qualquer vértice numa cópia de $T_{k}$ envia exatamente uma aresta para o conjunto $A$, então tem-se no mínimo mais duas arestas $x_{0} x_{1}$ e $x_{2} x_{3}$, uma em $T_{k}^{i_{1}}$ e outra em $T_{k}^{i_{2}}$, para que se possa atingir novamente um vértice em $A$. Assim, num menor circuito, tem-se que $v_{2} \in A_{i_{1}} \cap A_{i_{2}}$, e as arestas $x_{0} v_{2}$ e $x_{3} v_{2}$ fazem parte dos respectivos emparelhamentos (Figura 3.2).

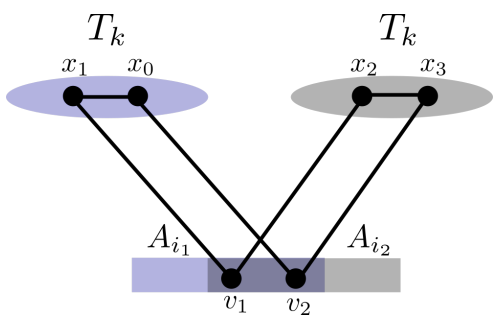

Figura 3.2: Os grafos de Tutte tem cintura pelo menos 6 .

Denote por $\left|T_{k}\right|$ o número de vértices de $T_{k}$. Então tem-se que $\left|T_{k}\right|$ satisfaz a recorrência

$$
\left|T_{k+1}\right|=k\left(\left|T_{k}\right|-1\right)+1+\left|T_{k}\right|\left(\begin{array}{c}
k\left(\left|T_{k}\right|-1\right)+1 \\
\left|T_{k}\right|
\end{array}\right) \geq k !
$$

Assim, essa construção possui mais vértices que o valor polinomial garantido pela demonstração probabilística. O valor $\left|T_{k+1}\right|$ será comparado com o tamanho das demais construções. Será visto que o grafo de Tutte em $\mathcal{G}_{k, 3}$ tem mais vértices que o grafo de Zykov em $\mathcal{G}_{k, 3}$, que é apresentado a seguir.

\section{$3.2 \quad$ O grafo de Zykov}

A construção de Zykov [39] para $Z_{k} \in \mathcal{G}_{k, 3}$ é indutiva. Para $k=1$, defina $Z_{1}$ como o grafo com um único vértice. Seja $k \geq 1$ e suponha que $Z_{k} \in \mathcal{G}_{k, 3}$ foi construído. Para construir $Z_{k+1,3}$, tome $k$ cópias disjuntas de $Z_{k}$ e tome um conjunto independente de vértices $A$ de tamanho $\left|Z_{k}\right|^{k}$. Considere todos os conjuntos de vértices $\left\{x_{1}, \ldots, x_{k}\right\}$ tal que cada vértice $x_{i}$, com $i \in[k]$, pertence a uma cópia distinta de $Z_{k}$. O número de conjuntos dessa forma é $\left|Z_{k}\right|^{k}$, então para cada vértice $v \in A$, associe um conjunto $\left\{x_{1}, \ldots, x_{k}\right\}$ adicionando as arestas entre $v$ e cada $x_{i}$ (Figura 3.3).

O grafo $Z_{k+1}$ é livre de triângulos. Dado que $A$ é independente, os vizinhos de um vértice $v$ em $A$ estão em cópias de $Z_{k}$. Além disso, $v$ não envia mais de uma aresta para uma mesma cópia, de modo que quaisquer dois de seus vizinhos $x_{1}$ e $x_{2}$ estão em cópias distintas de $Z_{k}$. Como as cópias de $Z_{k}$ são disjuntas, então $x_{1}$ e $x_{2}$ não estão conectados. Assim, os vértices de $A$ não fazem parte de nenhum triângulo. Por outro lado, por hipótese de indução, nenhuma cópia de $Z_{k}$ contém triângulo. 


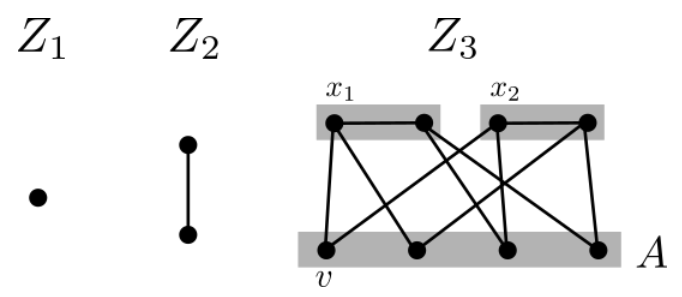

Figura 3.3: Construção de Zykov.

O número cromático de $Z_{k+1}$ é $k+1$. Suponha por contradição que existe uma coloração própria de $Z_{k+1}$ com $k$ cores. Cada cópia de $Z_{k}$ deve usar todas as $k$ cores por hipótese de indução. Tome $x_{i}$, com $i \in[k]$, um vértice em cada uma das $k$ cópias de $Z_{k}$ e tais que a cor de $x_{i}$ seja $i$. Existe um vértice $v \in A$ que está associado ao conjunto $\left\{x_{1}, \ldots, x_{k}\right\}$ e, portanto, é adjacente a cada $x_{i}$ do conjunto. Como $v$ foi colorido com alguma das $k$ cores, existe uma aresta monocromática, uma contradição. Por outro lado, $k+1$ cores são suficientes. Basta colorir cada cópia de $Z_{k}$ com as cores em $[k]$ usando a hipótese de indução e usar a cor $k+1$ para os vértices em $A$.

Denote por $\left|Z_{k}\right|$ o número de vértices de $Z_{k}$. O número de vértices dessa construção é dado pela recorrência

$$
\begin{aligned}
\left|Z_{k+1}\right| & =\left|Z_{k}\right|^{k}+k\left|Z_{k}\right| \\
& \geq k\left|Z_{k}\right| \geq k ! .
\end{aligned}
$$

Assim, o número de vértices não é polinomial em $k$. Porém, tem-se que o número de vértices dessa construção é menor que o da construção de Tutte.

Retomando a recorrência de Tutte, tem-se

$$
\begin{aligned}
\left|T_{k+1}\right| & =k\left(\left|T_{k}\right|-1\right)+1+\left|T_{k}\right|\left(\begin{array}{c}
k\left(\left|T_{k}\right|-1\right)+1 \\
\left|T_{k}\right|
\end{array}\right) \\
& \geq k\left|T_{k}\right|-k+1+\left|T_{k}\right| k^{\left|T_{k}\right|}\left(1-\frac{1}{\left|T_{k}\right|}\right)^{\left|T_{k}\right|} .
\end{aligned}
$$

Comparando com a recorrência de $\left|Z_{k}\right|$, como $-k+1+k^{\left|T_{k}\right|}\left(1-\frac{1}{\left|T_{k}\right|}\right)^{\left|T_{k}\right|}>0$, para $k \geq 3$, basta mostrar que

$$
\left(\left|T_{k}\right|-1\right) k^{\left|T_{k}\right|}\left(1-\frac{1}{\left|T_{k}\right|}\right)^{\left|T_{k}\right|} \geq\left|T_{k}\right|^{k}
$$

para concluir que a quantidade de vértices do grafo de Tutte é maior que a do grafo de Zykov.

Em termos gerais, como $\lim _{x \rightarrow \infty}\left(1-\frac{1}{x}\right)^{x}=e^{-1}$, então se $\left|T_{k}\right|$ é grande, vale que

$$
\left(\left|T_{k}\right|-1\right) k^{\left|T_{k}\right|}\left(1-\frac{1}{\left|T_{k}\right|}\right)^{\left|T_{k}\right|} \geq k^{\left|T_{k}\right|} .
$$

Além disso, quando $b \geq a \geq 3$, vale que $a^{b} \geq b^{a}$. Então $k^{\left|T_{k}\right|} \geq\left|T_{k}\right|^{k}$.

O número mínimo de vértices de um $\mathcal{G}_{3,3}$ é 5 , porque não pode ser bipartido e o menor circuto ímpar, que não o triângulo, é o $C_{5}$. O grafo $Z_{3}$ tem 8 vértices. Com relação ao menor grafo em $\mathcal{G}_{4,3}$, Chvátal [10] provou que o menor número de vértices é 11 e que o grafo que o satisfaz é único, o grafo de Grötzsch. O grafo $Z_{4}$ tem 536 vértices, mas o grafo de Mycielski em $\mathcal{G}_{4,3}$ será o grafo de 
Grötzsch, como será visto a seguir.

\subsection{O grafo de Mycielski}

A construção do grafo de Mycielski [28] também é indutiva em $k$. Denote por $M_{k}$ o grafo de Mycielski em $\mathcal{G}_{k, 3}$. Defina $M_{1}$ como grafo de um único vértice; a construção se inicia com $M_{2}=K_{2}$. Tendo construído $M_{k}$, para obter $M_{k+1}$, tome uma cópia de $M_{k}$ e, para cada vértice $v \in V\left(M_{k}\right)$, tome um novo vértice $v^{\prime}$. O vértice $v^{\prime}$ será chamado de vértice irmão de $v$. Seja $N(v)$ o conjunto dos vizinhos de $v$ na cópia de $M_{k}$. Adicione todas as arestas entre $v^{\prime}$ e $N(v)$. Por enquanto, $v$ e seu vértice irmão $v^{\prime}$ compartilham os mesmos vizinhos, todos na cópia de $M_{k}$. Enfim adicione um novo vértice $w$ e ligue-o a todos os vértices irmãos. Esse grafo é $M_{k+1}$ (Figura 3.4).

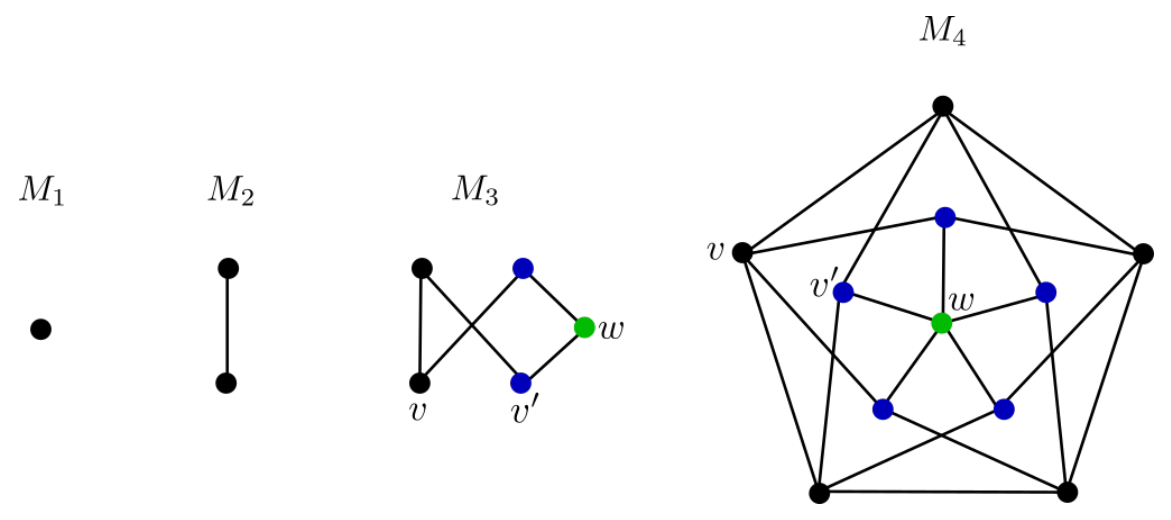

Figura 3.4: Construção de Mycielski. Tem-se que $M_{3}$ é $C_{5}$ e que $M_{4}$ é o grafo de Grötzsch.

O grafo construído não contém triângulos. Se houvesse algum, ele não poderia conter o vértice $w$, já que seus vizinhos são os vértices irmãos e não há arestas entre vértices irmãos. Esse triângulo também não poderia conter nenhum vértice irmão $v^{\prime}$, pois a vizinhança de $v^{\prime}$, exceto $w$, é a mesma de algum vértice $v$ da cópia de $M_{k}$ e uma aresta nessa vizinhança também determinaria um triângulo em $M_{k}$. Por fim, o triângulo não pode ter apenas vértices da cópia de $M_{k}$ por hipótese.

E vale que $\chi\left(M_{k+1}\right)=k+1$. Suponha por contradição que existe uma coloração própria de $V\left(M_{k+1}\right)$ com as cores em $[k]$. Então a cor de $w$ não pode ter sido atribuída a nenhum vértice irmão, sem perda de generalidade essa cor é $k$. Pela hipótese de indução, todas as $k$ cores foram utilizadas para colorir os vértices da cópia de $M_{k}$. Defina, então, uma coloração de $M_{k}$ que seja igual à coloração da cópia de $M_{k}$, exceto pelos vértices $v$ da cópia que receberam a cor $k$. A nova coloração troca a cor de $v$ pela cor de seu irmão $v^{\prime}$. Essa coloração de $M_{k}$ deve ser própria, basta verificar que a cor de $v^{\prime}$ não pode ser igual à cor de nenhum vizinho de $v$ em $M_{k}$. De fato, esses vizinhos constituem $N(v)$ que, por construção, também são vizinhos de $v^{\prime}$. Dessa forma, consegue-se uma coloração própria de $M_{k}$ com $k-1$ cores, o que é uma contradição. Por outro lado, é simples ver que $k+1$ cores são suficientes para uma coloração própria. Primeiro, tome uma coloração própria da cópia de $M_{k}$ com $k$ cores, que existe por hipótese. Depois, para cada vértice da cópia, pinte seu irmão com a mesma cor. Por fim, atribua uma cor nova a $w$.

Essa construção, de fato, produz muito menos vértices que a de Tutte ou a de Zykov. Tem-se 
que

$$
\begin{aligned}
\left|M_{k+1}\right| & =2\left|M_{k}\right|+1 \\
& =1+2+\cdots+2^{k-2}+2^{k-1}\left|M_{2}\right|=2^{k}+2^{k-1}-1
\end{aligned}
$$

a quantidade é exponencial em $\mathrm{k}$.

\subsection{Os grafos de shift}

Outra construção de $\mathcal{G}_{k, 3}$ é dada pelos grafos de shift [15]. O número de vértices dessa construção também é exponencial em $k$, mas ela não é indutiva.

Para $n \geq 3$, o grafo de shift $S_{n}$ tem como vértices as duplas $(i, j)$ com $1 \leq i<j \leq n$. Um par $\{(i, j),(k, l)\}$ de vértices forma uma aresta se $j=k$ (Figura 3.5).

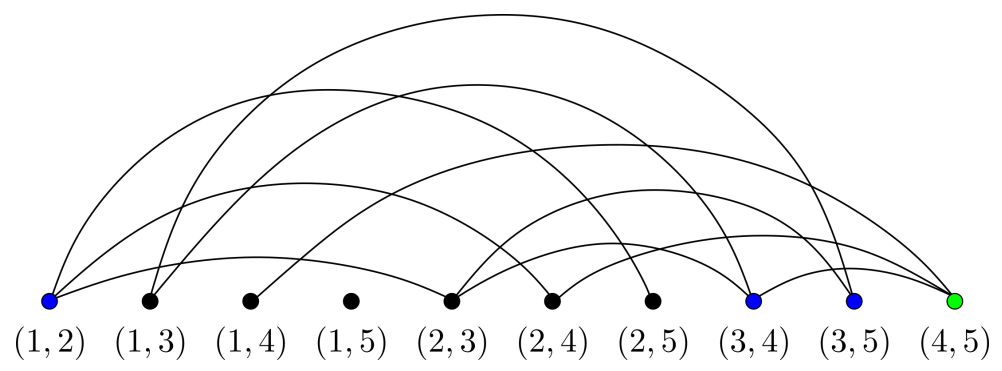

Figura 3.5: $O$ grafo $S_{5}$ tem número cromático 3 .

Percebe-se que $S_{n}$ não contém triângulos. Se $((i, j),(j, k),(k, l))$ determinasse um triângulo, seria preciso que $i<j<k<l$ ao mesmo tempo em que $\{(i, j),(k, l)\}$ ou $\{(k, l),(i, j)\}$ são arestas. Se algum desses pares fosse aresta, então $j=k$ ou $l=i$, que é uma contradição. Sobre o número cromático dos grafos de shift, será provado o seguinte resultado.

Proposição 3.4.1. Para todo $n \geq 3$, tem-se $\chi\left(S_{n}\right)=\left\lceil\log _{2} n\right\rceil$.

Demonstração. Para essa demonstração, define-se para certo digrafo $G$, o seu grafo linha $L(G)$. O conjunto de vértices de $L(G)$ é o conjunto de arcos de $G$. Uma dupla $\{(i, j),(k, l)\}$ de vértices de $L(G)$ formam uma aresta se $j=k$. Assim, uma aresta em $L(G)$ corresponde a um caminho orientado de tamanho 2 em $G$.

Repare que, dado um $K_{n}$ orientado transitivamente, i.e., com vértices $\{1, \ldots, n\}$ e $\operatorname{arcos}(i, j)$ para todo $i<j \in[n]$, seu grafo linha é $L\left(K_{n}\right)=S_{n}$. Para qualquer digrafo $G$, tem-se $\chi(L(G)) \geq$ $\left\lceil\log _{2} \chi(G)\right\rceil$. Para ver isso, seja $k=\chi(L(G))$ e considere uma coloração própria de $L(G)$ com $k$ cores. Então, considerando $G$, foi definida uma coloração dos arcos de $G$. Nessa coloração, para todo vértice $v \in V(G)$, a cor de qualquer arco que entra em $v$ é diferente da cor de qualquer arco que sai de $v$. Atribua a cada $v \in V(G)$ o conjunto $A_{v}$ das cores dos arcos que saem de $v$. Os conjuntos $A_{v}$ são cores e essa coloração de $V(G)$ é própria. Para qualquer aresta $(u, v)$, vale que $A_{u} \neq A_{v}$, uma vez que a cor de $(u, v)$ deve estar contida em $A_{u}$, mas não pode estar contida em $A_{v}$. Além disso, essa coloração usa no máximo $2^{k}$ cores, portanto, $2^{\chi(L(G))} \geq \chi(G)$.

Por outro lado, consegue-se colorir propriamente $L\left(K_{n}\right)$ com $k=\left\lceil\log _{2} n\right\rceil$ cores. Considere o $K_{n}$ orientado e todos os subconjuntos de $[k]$. O número de subconjuntos de $[k]$ é $2^{k} \geq n$. A cada vértice de $K_{n}$ será associado um subconjunto. Ao vértice 1 , associe o subconjunto que contém todos os 
elementos; ele será $A_{1}$. Ao vértice 2 , associe um subconjunto de $k-1$ elementos; ele será $A_{2}$. Siga, na ordem dos vértices, atribuindo a cada um, um maior subconjunto que ainda não foi associado a nenhum vértice.

Colorir $L\left(K_{n}\right)$ propriamente é o mesmo que colorir os arcos de $K_{n}$ sem que exista caminho orientado de tamanho 2 monocromático. Assim, os arcos de $K_{n}$ serão coloridos conforme os conjuntos que foram associados aos seus extremos. Um arco que saia de $v \in[n]$ irá receber uma cor de $A_{v}$. Além disso, todos os arcos que entram em $v$ receberão uma cor que não está contida em $A_{v}$. Seja $(u, v)$ um arco de $K_{n}$. Como $u<v$, o conjunto $A_{u}$ foi atribuído antes de $A_{v}$, portanto vale que $\left|A_{u}\right| \geq\left|A_{v}\right|$. Se $\left|A_{u}\right|>\left|A_{v}\right|$, existe cor em $A_{u} \backslash A_{v}$; se $\left|A_{u}\right|=\left|A_{v}\right|$, como são distintos, também existe cor em $A_{u} \backslash A_{v}$. Atribua a $(u, v)$ uma cor de $A_{u} \backslash A_{v}$. Faça isso para todo arco que entra em $v$. Qualquer cor de $A_{v}$ ainda pode ser utilizada para colorir as arestas que saem de $v$ sem criar caminho orientado de comprimento 2 monocromático. Escolha outro vértice e repita o procedimento para todas as arestas que entram nele. Tem-se uma coloração própria de $L\left(K_{n}\right) \operatorname{com}\left\lceil\log _{2} n\right\rceil \operatorname{cores}$.

Um grafo de shift $S_{n}$ é um $\mathcal{G}_{k, 3}$ quando $\left\lceil\log _{2} n\right\rceil \geq k$. Dessa forma, para $n=2^{k-1}+1$, vale que $S_{n} \in \mathcal{G}_{k, 3}$. O número de vértices desse grafo é

$$
\left|S_{2^{k-1}+1}\right|=\left(\begin{array}{c}
2^{k-1}+1 \\
2
\end{array}\right)=2^{2 k-3}+2^{k-2} .
$$

Para o grafo de Mycielski, tem-se $\left|M_{k}\right|=2^{k-1}+2^{k-2}-1$. Portanto, para $k \geq 2$, o grafo de shift em $\mathcal{G}_{k, 3}$ tem mais vértices que o de Mycielski.

\subsection{O grafo de Kneser}

Kneser [19] estudava os subconjuntos de $[n]$ de tamanho $t$ quando se interessou em saber como particionar esses subconjuntos de forma que, numa mesma parte, quaisquer dois subconjuntos sempre se intersectassem. Ele inclusive conjecturou um certo valor para o número mínimo de partes necessárias. A questão que despertou o interesse de Kneser pode ser reformulada a partir da definição do grafo de Kneser.

Definição 3.5.1. Sejam $n$ e $t$ inteiros positivos, com $n \geq t$. O grafo de Kneser $K(n, t)=(V, E)$ tem

- $V=\left(\begin{array}{c}{[n]} \\ t\end{array}\right)$, o conjunto de todos subconjuntos de $[n]$ de tamanho $t$ e

- $E=\{\{u, v\}: u \cap v=\emptyset\}$.

Nesse grafo, os subconjuntos de $[n]$ de tamanho $t$ são os vértices. E uma coleção de subconjuntos tais que quaisquer dois deles sempre se intersectam é uma coleção de vértices independentes. Dessa forma, particionar os subconjuntos de forma que quaisquer dois em uma mesma parte sempre se intersectem é igual particionar os vértices de $K(n, t)$ em conjuntos independentes. O número mínimo de conjuntos independentes que particiona os vértices de $K(n, t)$ é seu número cromático, já que cada cor corresponde a um conjunto independente.

A Conjectura de Kneser é que $\chi(K(n, t))=n-2 t+2$, para $n \geq 2 t-1$. Quando $n=2 t-1 \mathrm{e}$, na verdade, sempre que $n<2 t$, tem-se que $K(n, t)$ não possui arestas, pois dois subconjuntos de 
tamanho $t$ se intersectam necessariamente. Portanto, o grafo pode ser colorido com uma única cor. Considere, a partir de agora, que $n \geq 2 t$.

Quando $t=1$, a conjectura é de que $\chi(K(n, 1))=n$. De fato, o grafo $K(n, 1)$ é o clique de $n$ vértices, já que dois subconjuntos unitários distintos nunca se intersectam. Para valores de $t$ em geral, pode-se determinar uma coloração de $K(n, t) \operatorname{com} n-2 t+2$ cores.

Proposição 3.5.1. Sejam $n$ et inteiros positivos, com $n \geq 2 t-1$. Vale que $\chi(K(n, k)) \leq n-2 t+2$.

Demonstração. Será apresentada uma coloração própria de $K(n, t)$ com cores em $[n-2 t+2]$.

Observe que, se os subconjuntos de tamanho $t$ forem tomados de um conjunto de tamanho $2 t-1$, então eles se intersectam. Dessa forma, os vértices contidos em $[n-(2 t-2), n]$ podem ser coloridos com uma única cor. Será a cor $n-2 t+2$.

Os vértices ainda não coloridos são aqueles que contém algum número em $[1, n-(2 t-1)]$. Assim, tem-se que o menor elemento de um vértice não colorido pertence a $[1, n-(2 t-1)]$. Note que, fixando um número $i \in[1, n-(2 t-1)]$, todos os vértices que contém $i$ formam um conjunto independente. Portanto, use a cor $i \in[1, n-(2 t-1)]$ para colorir os vértices cujo menor elemento seja $i$. Esta coloração é própria e utiliza $n-2 t+2$ cores (Figuras 3.6 e 3.7).

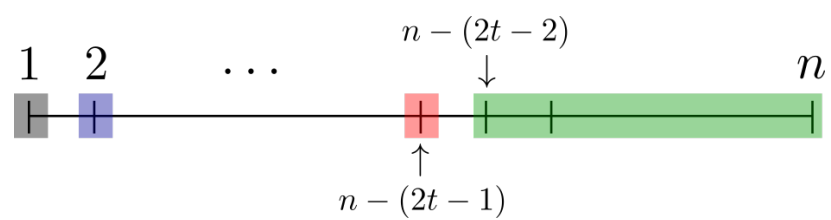

Figura 3.6: Os vértices são coloridos segundo seu menor elemento.

A Conjectura de Kneser foi provada primeiro por Lovász [25], utilizando um teorema da Topologia, o Teorema de Borsuk-Ulam. Pouco tempo depois, Bárány [6] combinou o Teorema de Borsuk-Ulam ao Lema de Gale para uma demonstração mais elegante. Então Greene [16] simplificou ainda mais a demonstração, eliminando a necessidade do Lema de Gale. Assim, o número cromático do grafo de Kneser foi determinado.

Antes de apresentar uma demonstração que determina $\chi(K(n, t))=n-2 t+2$, note que, sendo a conjectura válida, o grafo de Kneser pode ser utilizado para se obter um grafo em $\mathcal{G}_{k, 3}$. Para conseguir $\chi(K(n, t)) \geq k$, basta que $n \geq k+2 t-2$. Para evitar triângulos, basta tomar $3 t>n$, de modo que 3 subconjuntos de tamanho $t$ não poderão ser dois a dois disjuntos. Assim, para que exista um valor possível para $n$, é preciso que $t \geq k-1$.

Para $k=3$, por exemplo, pode-se tomar $t=2$ e, necessariamente, $n=5$. O grafo $K(5,2) \in \mathcal{G}_{3,3}$ é o grafo de Petersen. Ele tem número cromático 3 e não possui triângulos. A Figura 3.7 apresenta uma coloração própria de $K(5,2)$, conforme a descrita na demonstração da Proposição 3.5.1.

Agora se apresenta a demonstração de Greene, que também pode ser lida em [2], da Conjectura de Kneser. Como $n \geq 2 t$, a conjectura é reformulada escrevendo-se $n=2 t+d$.

Teorema 3.5.2. Seja $n=2 t+d$, com $t \geq 1$ e $d \geq 0$. Vale que $\chi(K(n, t))=n-2 t+2=d+2$.

Demonstração. Para a demonstração da Conjectura de Kneser, os elementos do conjunto [n] são identificados com pontos na esfera $S^{d+1}=\left\{x \in \mathbb{R}^{d+2}:|x|=1\right\}$. Em seguida, utiliza-se a versão do Teorema de Borsuk-Ulam enunciada a seguir. 


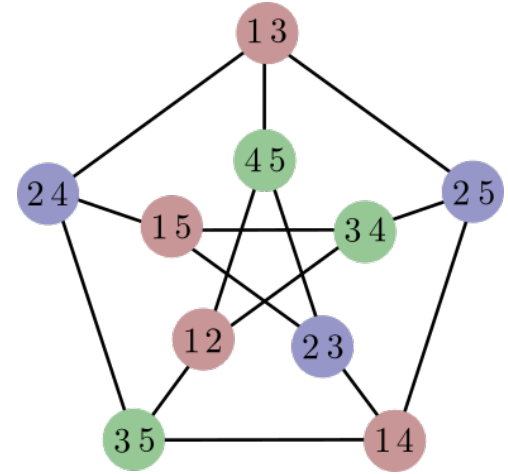

Figura 3.7: $O$ grafo $K(5,2)$ é o grafo de Petersen.

Teorema 3.5.3. Se $S^{d}$ é coberta por $d+1$ conjuntos, $S^{d}=U_{1} \cup \cdots \cup U_{d+1}$, com cada $U_{i}(1 \leq i \leq d)$ aberto ou fechado, então algum desses $d+1$ conjuntos contém pontos antípodas $x^{*} e-x^{*}$.

O Teorema 3.5.3 não é o Teorema de Borsuk-Ulam usual [9]. Essa versão pode ser provada a partir de Borsuk-Ulam. Mas na verdade, a primeira demonstração do Teorema 3.5.3 é mais antiga que o Teorema de Borsuk-Ulam e se deve a Lyusternik e Shnirel'man [27]. Essa primeira demonstração considerava que todos $U_{i}(1 \leq i \leq d+1)$ eram fechados. Greene notou que o teorema também valia quando cada $U_{i}(1 \leq i \leq d+1)$ era aberto ou fechado. A versão apresentada está segundo Aigner e Ziegler [2] e elimina a necessidade de suposição sobre $U_{d+1}$. O Teorema 3.5.3 não será provado.

Continua-se a demonstração do Teorema 3.5.2. Considere $P$ uma coleção de $2 t+d$ pontos em posição geral sobre $S^{d+1}$. Estar em posição geral significa que qualquer hiperplano que passe pela origem pode conter no máximo $d+1$ desses pontos. Enumere cada ponto de $P$ com um elemento de $[2 t+d]$. Tem-se que os subconjuntos de $P$ com tamanho $t$ correspondem a vértices de $K(2 t+d, t)$.

Considere uma partição qualquer dos vértices de $K(2 t+d, t)$ em $d+1$ conjuntos $V_{1}, \ldots, V_{d+1}$. Para mostrar que $\chi(K(2 t+d, t)) \geq d+2$, é preciso que alguma dessas partes contenha uma aresta. Por isso, buscam-se dois subconjuntos de $P$ de tamanho $t$ que sejam disjuntos e estejam associados a vértices contidos numa mesma parte.

Será definida uma cobertura de $S^{d+1}$ por $d+2$ conjuntos, para que se possa aplicar o Teorema 3.5.3. Para isso defina, para cada ponto $x \in S^{d+1}$, o hemisfério aberto

$$
H_{x}=\left\{y \in S^{d+1}:\langle x, y\rangle>0\right\}
$$

Agora observe os pontos de $P$ contidos em cada $H_{x}$. Se $H_{x}$ contém algum subconjunto de $P$ de tamanho $t$, como cada um destes subconjuntos corresponde a um certo vértice $v$ de $K(2 t+d, t)$, então será dito que $H_{x}$ contém $v$. Esse vértice $v$ deve pertencer a alguma parte $V_{i}$. Assim, definem-se os conjuntos

$$
O_{i}=\left\{x \in S^{d+1}: \exists v \in\left(\begin{array}{c}
P \\
t
\end{array}\right) \text { tal que } v \subset H_{x} \text { e } v \in V_{i}\right\} .
$$

Assim, $O_{i}$ é formado por cada ponto da esfera cujo hemisfério contenha algum vértice que esteja na parte $V_{i}$ (Figura 3.8). Note que cada conjunto $O_{i}$ é aberto.

Tomando $C=S^{d+1} \backslash \bigcup_{1 \leq i \leq d+1} O_{i}$, fica definida a cobertura $S^{d+1}=O_{1} \cup \cdots \cup O_{d+1} \cup C$. Como $C$ é fechado, não é realmente necessário utilizar a versão mais forte do Teorema 3.5.3, basta a versão de Greene. Assim, utilizando o Teorema 3.5.3, conclui-se que algum dos conjuntos da cobertura 


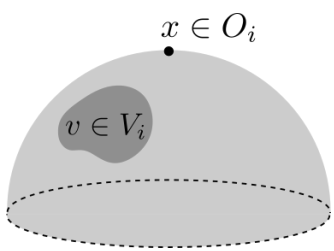

Figura 3.8: $O$ ponto $x$ pertence $O_{i}$ quando o hemisfério $H_{x}$ contém um vértice $v \in V_{i}$.

deve conter pontos antípodas $x^{*}$ e $-x^{*}$. Suponha por contradição que $\left\{x^{*},-x^{*}\right\} \subset C$. Para $x^{*}$ e $-x^{*}$ pertencerem a $C$, os hemisférios $H_{x^{*}}$ e $H_{-x^{*}}$ podem conter no máximo $t-1$ pontos em $P$. Ainda sobram pelo menos $d+2$ pontos, que devem estar em $\left\{y \in S^{d+1}:\left\langle x^{*}, y\right\rangle=0\right\}$, o equador. Mas o hiperplano $\left\{y \in \mathbb{R}^{d+2}:\left\langle x^{*}, y\right\rangle=0\right\}$, que passa pelo equador e também centro, pode conter no máximo $d+1$ pontos de $P$.

Assim, $x^{*}$ e $-x^{*}$ devem pertencer a algum $O_{i}$. Isso significa que existem vértices $v_{1}$ e $v_{2}$ pertencentes à parte $V_{i}$, tais que $v_{1} \subset H_{x^{*}}$ e $v_{2} \subset H_{-x^{*}}$. Como os hemisférios $H_{x^{*}}$ e $H_{-x^{*}}$ são disjuntos, tem-se que $v_{1} \cap v_{2}=\emptyset$ (Figura 3.9). Dessa forma, encontra-se uma aresta em $V_{i}$.

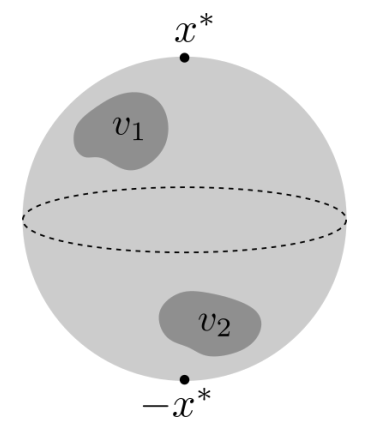

Figura 3.9: Existem vértices disjuntos contidos numa mesma parte.

O número de vértices de $K(n, t)$ é o número de subconjuntos de $[n]$ de tamanho $t$, que é $\left(\begin{array}{l}n \\ t\end{array}\right)$. Como foi dito, é preciso escolher corretamente os valores $n_{k}$ e $t_{k}$ para que $K\left(n_{k}, t_{k}\right)$ seja um grafo em $\mathcal{G}_{k, 3}$. Para se ter $\chi\left(K\left(n_{k}, t_{k}\right)\right)=k$, tome $n_{k}=k+2 t_{k}-2$. Para que $K\left(n_{k}, t_{k}\right)$ não contenha triângulos, tome $3 t_{k}>n_{k}$. Isso implica que $t_{k} \geq k-1$.

Pode-se estimar o número de vértices de $K\left(n_{k}, t_{k}\right)$. Tem-se

$$
\left(\begin{array}{l}
n_{k} \\
t_{k}
\end{array}\right)=\left(\begin{array}{c}
k+2 t_{k}-2 \\
t_{k}
\end{array}\right) \geq\left(2+\frac{k-2}{t_{k}}\right)^{t_{k}} \geq 2^{k-1}
$$

onde a última desigualdade vale, pois $t_{k} \geq k-1$ e $(k-2) / t_{k}>0$.

Lembre-se que a construção de Mycielski de um grafo $M_{k} \in \mathcal{G}_{k, 3}$ tem $2^{k}+2^{k-1}-1=3 \cdot 2^{k-1}-1$ vértices. Será visto que é possível aumentar a base da exponencial da cota do número de vértices de $K\left(n_{k}, t_{k}\right)$. Assim, será provado que para $k \geq 10$, o grafo $K\left(n_{k}, t_{k}\right)$ possui mais vértices que $M_{k}$. Para isso, separe os casos em que $t_{k}$ é maior que $2(k-1)$ e os em que $t_{k}$ é no máximo esse valor.

Se $t_{k}>2(k-1)$, então

$$
\left(\begin{array}{l}
n_{k} \\
t_{k}
\end{array}\right) \geq\left(2+\frac{k-2}{t_{k}}\right)^{t_{k}}>2^{2(k-1)}=4^{k-1}
$$


Se $t_{k} \leq 2(k-1)$, então

$$
\left(\begin{array}{l}
n_{k} \\
t_{k}
\end{array}\right) \geq\left(2+\frac{k-2}{t_{k}}\right)^{t_{k}} \geq\left(2+\frac{k-2}{2(k-1)}\right)^{k-1} \geq\left(2+\frac{1}{4}\right)^{k-1},
$$

onde a última desigualdade vale, pois $x /(x+1) \geq 1 / 2$ para $x \geq 1$.

Como nenhum dos dois casos é vazio, o número de vértices do grafo $K\left(n_{k}, t_{k}\right)$ é

$$
\left(\begin{array}{l}
n_{k} \\
t_{k}
\end{array}\right) \geq \min \left\{4^{k-1},\left(2+\frac{1}{4}\right)^{k-1}\right\}=\left(\frac{9}{4}\right)^{k-1} .
$$

Comparando com Mycielski, para $(9 / 4)^{k-1} \geq 3 \cdot 2^{k-1}-1$, basta $(9 / 8)^{k-1} \geq 3$, que vale para $k \geq 10$.

O número de vértices do grafo de shift em $\mathcal{G}_{k, 3}$ é

$$
\left|S_{2^{k-1}+1}\right|=\left(\begin{array}{c}
2^{k-1}+1 \\
2
\end{array}\right)=\frac{4^{k-1}+2^{k-1}}{2} .
$$

Assim, é preciso melhorar ainda mais a cota do número de vértices de $K\left(n_{k}, t_{k}\right)$ para que seu tamanho possa ser comparado com o de $S_{2^{k-1}+1}$. Será utilizada a aproximação de Stirling para o fatorial, segundo a qual

$$
\sqrt{2 \pi}\left(\frac{n}{e}\right)^{n} \sqrt{n} \leq n ! \leq e\left(\frac{n}{e}\right)^{n} \sqrt{n} .
$$

O número de vértices de $K\left(n_{k}, t_{k}\right)$ é

$$
\left(\begin{array}{c}
n_{k} \\
t_{k}
\end{array}\right)=\left(\begin{array}{c}
k+2 t_{k}-2 \\
t_{k}
\end{array}\right)=\frac{\left(k+2 t_{k}-2\right) !}{t_{k} !\left(k+t_{k}-2\right) !} .
$$

Utilizando a aproximação de Stirling, tem-se

$$
\begin{aligned}
\left(\begin{array}{c}
n_{k} \\
t_{k}
\end{array}\right) & \geq \frac{\sqrt{2 \pi}}{e^{2}} \sqrt{\frac{k+2 t_{k}-2}{t_{k}\left(k+t_{k}-2\right)}} \frac{\left(k+2 t_{k}-2\right)^{k+2 t_{k}-2}}{t_{k}^{t_{k}}\left(k+t_{k}-2\right)^{k+t_{k}-2}} \\
& =\frac{\sqrt{2 \pi}}{e^{2}} \sqrt{\frac{k+2 t_{k}-2}{t_{k}\left(k+t_{k}-2\right)}}\left(\frac{k+2 t_{k}-2}{t_{k}}\right)^{t_{k}}\left(\frac{k+2 t_{k}-2}{k+t_{k}-2}\right)^{k+t_{k}-2} .
\end{aligned}
$$

Cada termo será analisado separadamente. Como $t_{k} \geq k-1$, tem-se

$$
\frac{k+2 t_{k}-2}{k+t_{k}-2} \geq \frac{3}{2}
$$

Além disso, tem-se

$$
\left(\frac{k+2 t_{k}-2}{t_{k}}\right)^{t_{k}}=\left(2+\frac{k-2}{t_{k}}\right)^{t_{k}} \geq 2^{t_{k}}+2^{t_{k}-1} t_{k} \frac{k-2}{t_{k}}=2^{t_{k}}\left(1+\frac{k-2}{2}\right)=2^{t_{k}-1} k .
$$

Dessa forma,

$$
\left(\begin{array}{l}
n_{k} \\
t_{k}
\end{array}\right) \geq \frac{\sqrt{3 \pi}}{e^{2}} \frac{1}{\sqrt{t_{k}}} 2^{t_{k}-1} k\left(\frac{3}{2}\right)^{k+t_{k}-2}=\frac{\sqrt{3 \pi}}{9 e^{2}} \frac{k}{\sqrt{t_{k}}} \frac{3^{k+t_{k}}}{2^{k-1}}
$$


A função $f(x)=\frac{3^{x}}{\sqrt{x}}$ é crescente, portanto pode-se fazer $t_{k}=k-1$. Tem-se

$$
\left(\begin{array}{l}
n_{k} \\
t_{k}
\end{array}\right) \geq \frac{\sqrt{3 \pi}}{9 e^{2}} \frac{k}{\sqrt{k-1}} \frac{3^{2 k-1}}{2^{k-1}} \geq \frac{\sqrt{3 \pi}}{9 e^{2}} \frac{2}{3} \sqrt{k}\left(\frac{9}{2}\right)^{k}
$$

Portanto, para $k \geq 9$, vale que

$$
\left|K\left(n_{k}, t_{k}\right)\right| \geq \frac{2 \sqrt{3 \pi}}{27 e^{2}} \sqrt{k}\left(\frac{9}{2}\right)^{k} \geq 4^{k-1} \geq\left|S_{2^{k-1}+1}\right| .
$$

De modo que o grafo de Kneser em $\mathcal{G}_{k, 3}$ tem mais vértices que o grafo de shift em $\mathcal{G}_{k, 3}$.

Uma cota superior para o número de vértices do menor grafo de Kneser em $\mathcal{G}_{k, 3}$ pode ser facilmente obtida calculando-se $|K(k+2(k-1)-2, k-1)|$. Tem-se

$$
\left(\begin{array}{c}
k+2(k-1)-2 \\
k-1
\end{array}\right) \leq\left(\frac{e(k+2(k-1)-2)}{k-1}\right)^{k-1}=\left(e\left(2+\frac{k-2}{k-1}\right)\right)^{k-1} \leq 9^{k-1} .
$$

Dessa forma, o número de vértices do menor grafo de Kneser em $\mathcal{G}_{k, 3}$ é exponencial. Portanto, menor que o número de vértices dos grafos de Tutte e Zykov em $\mathcal{G}_{k, 3}$.

\subsection{Construção a partir de um plano projetivo}

Existem, ainda, construções com número de vértices polinomial em $k$. Uma delas é feita a partir do grafo de incidência de um plano projetivo finito [11].

Definição 3.6.1. Dados um conjunto finito $X$ e uma família $\mathcal{L}$ de subconjuntos de $X$, o par $(X, \mathcal{L})$ é um plano projetivo finito quando são satisfeitas as seguintes condições.

1. existe um subconjunto $F \subset X$ com $|F|=4$ tal que, para todo $L \in \mathcal{L}$, vale que $|F \cap L| \leq 2$;

2. para quaisquer $L_{1} \neq L_{2} \in \mathcal{L}$, vale que $\left|L_{1} \cap L_{2}\right|=1$;

3. para quaisquer $x_{1} \neq x_{2} \in X$, existe exatamente um conjunto $L \in \mathcal{L}$ tal que $x_{1}, x_{2} \in L$.

Os elementos de $X$ são chamados pontos e os de $\mathcal{L}$ são retas. Nessa linguagem, as condições acima significam o seguinte. Segundo (1), existem 4 pontos tais que 3 deles nunca estão alinhados. A condição (2) significa que duas retas sempre se intersectam em exatamente 1 ponto. E (3), que dois pontos sempre determinam uma única reta.

Um exemplo de plano projetivo finito é o plano de Fano (Figura 3.10). Nele, tem-se 7 pontos e 7 retas. Note que cada reta consiste de 3 pontos e, por cada ponto, passam 3 retas. Essa regularidade, na verdade, não é particular a esse exemplo. Pode-se provar a seguinte proposição [32] .

Proposição 3.6.1. Se $(X, \mathcal{L})$ é um plano projetivo finito, então para quaisquer duas retas $L_{1}, L_{2} \in \mathcal{L}$, tem-se $\left|L_{1}\right|=\left|L_{2}\right|$.

Assim, fica bem definida a ordem de um plano projetivo finito, que é $n=|L|-1 \operatorname{com} L \in \mathcal{L}$. O plano de Fano tem ordem 2. Sabe-se que existem planos projetivos finitos para qualquer ordem da forma $n=p^{m}$, potência de primo.

Um plano projetivo finito tem ainda as seguintes propriedades. 


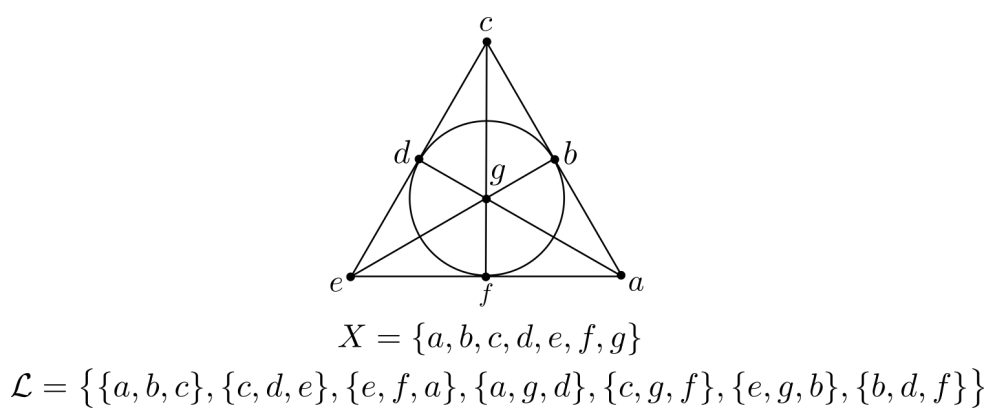

Figura 3.10: O plano de Fano.

Proposição 3.6.2. Se $(X, \mathcal{L})$ tem ordem $n$, então por cada ponto, passam exatamente $n+1$ retas. Além disso, tem-se $|X|=|\mathcal{L}|=n^{2}+n+1$.

No plano de Fano, que tem ordem 2, por cada ponto passam $n+1=3$ retas. Além disso, o número de pontos dele é $n^{2}+n+1=7$, que é igual ao seu número de retas.

Sabendo disso, define-se o grafo de incidência de um plano projetivo finito $I(X, \mathcal{L})=(V(I), E(I))$. O grafo $I$ tem vértices $V(I)=X \cup \mathcal{L}$ e é bipartido, sendo uma parte definida pelos pontos $X$ e a outra pelas retas $\mathcal{L}$. Um par $\{P, L\}$ é uma aresta de $I$ quando $P$ é um ponto que pertence à reta $L$. Como é bipartido, esse grafo não tem circuito ímpar. Ele também não contém circuito de comprimento 4, pois dois pontos determinam exatamente uma reta, logo, na vizinhança comum de dois vértices em $X$ não há mais de um vértice de $\mathcal{L}$. Portanto, esse grafo não contém circuito de comprimento menor que 6 .

O grafo buscado $P_{k}=\left(V\left(P_{k}\right), E\left(P_{k}\right)\right) \in \mathcal{G}_{k, 3}$ estará associado a um plano projetivo $(X, \mathcal{L})$ de ordem $n=n(k)$. Os vértices de $P_{k}$ são todos os pares $(P, L)$, com $P \in X$ um ponto da reta $L \in \mathcal{L}$. Para definir as arestas de $P_{k}$, é necessário tomar uma ordem total dos vértices $(P, L)$. Feito isso, define-se que $\left\{(P, L),\left(P^{\prime}, L^{\prime}\right)\right\}$, com $P \neq P^{\prime}$ e $L \neq L^{\prime}$, é uma aresta de $P_{k}$ se $(P, L)<\left(P^{\prime}, L^{\prime}\right)$ e $P \in L^{\prime}$. Para provar que $P_{k} \in \mathcal{G}_{k, 3}$, define-se um certo digrafo $H$, a partir do qual pode-se obter $P_{k}$. Serão provadas algumas propriedades de $H$. O digrafo $H$ será obtido a partir do grafo de incidência de $(X, \mathcal{L})$ e seu número de independência será limitado.

Assim, defina o digrafo $H=(V(H), A(H))$. Seus vértices são os mesmos de $P_{k}$, isto é, os pares $(P, L)$, com $P \in L$. Seus arcos são $\left((P, L),\left(P^{\prime}, L^{\prime}\right)\right)$, tais que $P \neq P^{\prime}$ e $L \neq L^{\prime}$ e $P \in L^{\prime}$. Não é difícil de notar que, se existir um triângulo em $H$, ele deverá ser orientado. Caso contrário, tem-se que um triângulo não orientado em $H$ determina um circuito de comprimento 4 em $I(X, \mathcal{L})$ (Figura 3.11).
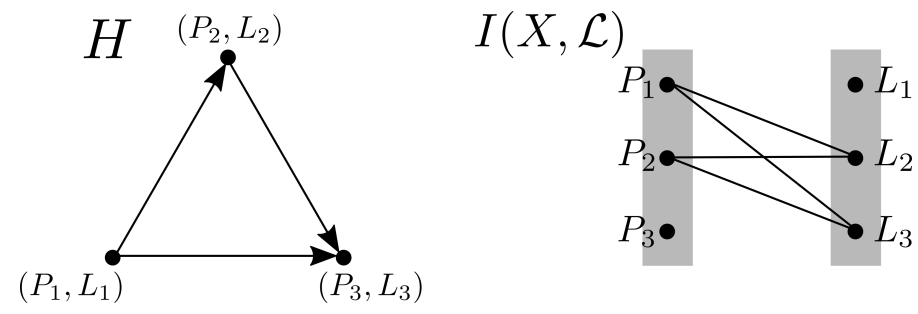

Figura 3.11: Um circuito não orientado em $H$ formaria um circuito de comprimento 4 no grafo de incidência.

Associe a $H$ uma matriz $M(H)$ com linhas e colunas indexadas pelos vértices $(P, L) \in V(H)$ 
em ordem crescente e com entradas

$$
M(H)_{(P, L),\left(P^{\prime}, L^{\prime}\right)}= \begin{cases}1, & (P, L)=\left(P^{\prime}, L^{\prime}\right) \\ -1, & \left((P, L),\left(P^{\prime}, L^{\prime}\right)\right) \in A(H) \\ 0, & \text { caso contrário. }\end{cases}
$$

Essa matriz tem a diagonal preenchida por 1. Além disso, afirma-se que se $N \subset V(H)$ é um conjunto independente em $H$, então o conjunto das colunas indexadas por $N$ é linearmente independente. Para cada $v \in N$, tem-se que $M(H)_{v, v}=1$. Para todos os outros $u \in N \backslash\{v\}$, tem-se $M(H)_{v, u}=0$. Desse modo, a entrada $v$ de qualquer combinação linear com coeficientes não nulos dessas colunas não será nula; as colunas indexadas por $N$ são linearmente independentes. Portanto, o posto de $M(H)$ é maior que ou igual ao tamanho do maior conjunto independente de $H$.

O próximo passo é ver que o posto de $M(H)$ é menor que ou igual a $2\left(n^{2}+n+1\right)$, limitando o tamanho do maior conjunto independente de $H$. Para isso, escreve-se $M(H)=A \cdot B$, o produto de duas matrizes. Define-se $A$ como uma matriz cujas linhas são indexadas por $V(H)$ em ordem crescente e as colunas por $V(I)$. Tem-se

$$
A_{(P, L), v}= \begin{cases}1, & v=L \\ -1, & v=P \\ 0, & \text { caso contrário. }\end{cases}
$$

A matriz $B$ tem as linhas indexadas por $V(I)$ e as colunas por $V(H)$ em ordem crescente. Define-se

$$
B_{v,(P, L)}= \begin{cases}1, & v=L \\ 1, & v \in L \backslash\{P\} \\ 0, & \text { caso contrário. }\end{cases}
$$

De fato, verifica-se que

$$
(A B)_{(P, L),\left(P^{\prime}, L^{\prime}\right)}=\sum_{v \in V(I)} A_{(P, L), v} B_{v,\left(P^{\prime}, L^{\prime}\right)}=B_{L,\left(P^{\prime}, L^{\prime}\right)}-B_{P,\left(P^{\prime}, L^{\prime}\right)} .
$$

Caso $L=L^{\prime}$, então $B_{L,\left(P^{\prime}, L^{\prime}\right)}=1$. Nesse caso, se $P=P^{\prime}$, então $B_{P,\left(P^{\prime}, L^{\prime}\right)}=0$. Isso significa que se tem 1 na diagonal. Caso $L \neq L^{\prime}$, então $B_{L,\left(P^{\prime}, L^{\prime}\right)}=0$. Nesse caso, se $P \neq P^{\prime}$ e $P \in L^{\prime}$, temse $B_{P,\left(P^{\prime}, L^{\prime}\right)}=1$. Isso significa que se tem -1 quando $\left((P, L),\left(P^{\prime}, L^{\prime}\right)\right)$ é um arco em $A(H)$. Pode-se verificar que, nos demais casos, $(A B)_{(P, L),\left(P^{\prime}, L^{\prime}\right)}=0$.

Então as colunas de $M(H)$ são combinações lineares das colunas de $A$. Dessa forma, o posto de $H$ é menor que ou igual ao número de colunas de $A$, que é igual a $|V(I)|=2\left(n^{2}+n+1\right)$.

Considere apenas o triângulo acima da diagonal de $M(H)$ e preencha o triângulo inferior de forma simétrica, chame essa nova matriz de $M\left(P_{k}\right)$. Como as linhas e colunas de $M\left(P_{k}\right)$ estão indexadas por $V(H)=V\left(P_{k}\right)$ em ordem crescente, pode-se notar que $M\left(P_{k}\right)$ está associada a $P_{k}$ 
da seguinte forma,

$$
M\left(P_{k}\right)_{(P, L),\left(P^{\prime}, L^{\prime}\right)}= \begin{cases}1, & (P, L)=\left(P^{\prime}, L^{\prime}\right) \\ -1, & \left\{(P, L),\left(P^{\prime}, L^{\prime}\right)\right\} \in E\left(P_{k}\right) \\ 0, & \text { caso contrário. }\end{cases}
$$

Assim, $P_{k}$ é um certo subdigrafo de $H$, do qual a orientação foi removida. Afirma-se que não há triângulos em $P_{k}$. Um triângulo em $P_{k}$ induziria um triângulo em $H$ com os mesmos vértices. Foi visto que $H$ só possui triângulos orientados. Todo triângulo orientado tem um arco da forma $\left(\left(P^{\prime}, L^{\prime}\right),(P, L)\right)$ com $\left(P^{\prime}, L^{\prime}\right)>(P, L)$. Se, por um lado, $\left(\left(P^{\prime}, L^{\prime}\right),(P, L)\right) \in A(H)$, então $P, P^{\prime} \in L$. Se, por outro lado, $\left\{\left(P^{\prime}, L^{\prime}\right),(P, L)\right\} \in E\left(P_{k}\right)$, como $\left(P^{\prime}, L^{\prime}\right)>(P, L)$, tem-se que $P, P^{\prime} \in L^{\prime}$. Mas apenas uma aresta pode conter $P$ e $P^{\prime}$, portanto $L=L^{\prime}$, uma contradição.

Além disso, afirma-se que as colunas de $M\left(P_{k}\right)$ correspondentes a um conjunto independente de $P_{k}$ corresponderão a colunas linearmente independentes de $M(H)$. Para ver isso, considere que os primeiros $a$ vértices sejam independentes em $P_{k}$. Então a matriz $M\left(P_{k}\right)$ tem um bloco de tamanho $a$ igual à identidade $I_{a}$. Na matriz $M(H)$, esse bloco de tamanho a pode ter entradas diferentes de 0 apenas abaixo da diagonal. Assim, em $M(H)$, nenhuma outra entrada na primeira linha do bloco, exceto a diagonal, será diferente de 0 . Logo, uma combinação linear nula das primeiras a colunas de $M(H)$ deve multiplicar a primeira coluna por 0. Como já se sabe que o coeficiente da primeira coluna deve ser 0, pode-se aplicar o mesmo raciocínio para a coluna seguinte. Assim, prova-se que as a primeiras colunas de $M(H)$ são linearmente independentes. Caso o conjunto independente não corresponda às primeiras a colunas, troque a ordem das colunas de $M(H)$ e correspondentemente a ordem das linhas. Faça-o de modo que as a primeiras colunas passem a corresponder ao conjunto independente e sem trocar a ordem relativa entre as colunas correspondentes ao conjunto independente. Assim, limita-se o tamanho do conjunto independente máximo de $P_{k}$, que deve ser menor que ou igual ao posto de $M(H)$.

Provou-se que $P_{k}$ não tem triângulo e que $\alpha\left(P_{k}\right) \leq 2\left(n^{2}+n+1\right)$. Pela Proposição 3.6.2, podese dizer que $\left|P_{k}\right|=\left(n^{2}+n+1\right)(n+1)$. Então $\chi\left(P_{k}\right) \geq(n+1) / 2$. Será determinado um valor para $n=n(k)$. Dado $k$ inteiro positivo, existe a potência de primo $k \leq 2^{\left\lceil\log _{2} k\right\rceil} \leq 2 k$. Tomando um plano projetivo finito de ordem $n=2^{\left\lceil\log _{2} k\right\rceil+1}$, com essa construção tem-se

$$
\chi\left(P_{k}\right) \geq \frac{n+1}{2} \geq \frac{2^{\left\lceil\log _{2} k\right\rceil+1}}{2} \geq k .
$$

Como $2 k \leq n \leq 4 k$, seu número de vértices é

$$
\left(4 k^{2}+2 k+1\right)(2 k+1) \leq\left|P_{k}\right| \leq\left(16 k^{2}+4 k+1\right)(4 k+1) .
$$

Para $n \geq 3$, vale que $\left(n^{2}+n+1\right)(n+1)=n^{3}+2 n^{2}+2 n+1 \leq 2 n^{3}$. Assim,

$$
\begin{gathered}
(2 k)^{3} \leq\left|P_{k}\right| \leq 2(4 k)^{3} \\
k^{3} \leq\left|P_{k}\right| \leq 16 k^{3}
\end{gathered}
$$

Essa é a menor construção; tem-se $\left|P_{k}\right|=\Theta\left(k^{3}\right)$. Porém ela não é elementar, pois utiliza planos projetivos finitos de diferentes ordens. 


\subsection{Consideração final}

Como foi visto ao longo do capítulo, construções para grafos livres de triângulos com número cromático grande podem ser obtidas de formas variadas. Comparando os números de vértices das construções apresentadas, tem-se o seguinte teorema.

Teorema 3.7.1. Sejam respectivamente $T_{k}, Z_{k}, M_{k}, S_{n_{k}}, K\left(n_{k}, t_{k}\right), P_{k}$ os grafos de Tutte, Zykov, Mycielski, shift, Kneser e planos projetivos finitos em $\mathcal{G}_{k, 3}$. Para $k$ grande, seus números de vértices em ordem decrescente são como apresentados a seguir.

1. $\left.\left|T_{k}\right|=k\left(\left|T_{k-1}\right|-1\right)+1+\left|T_{k-1}\right| \begin{array}{c}\left(\left|T_{k-1}\right|-1\right)+1 \\ \left|T_{k-1}\right|\end{array}\right)$,

2. $\left|Z_{k}\right|=\left|Z_{k-1}\right|^{k-1}+(k-1)\left|Z_{k-1}\right| \geq(k-1)$ !,

3. $9^{k-1} \geq\left|K\left(n_{k}, t_{k}\right)\right| \geq \frac{2 \sqrt{3 \pi}}{27 e^{2}} \sqrt{k}\left(\frac{9}{2}\right)^{k}$,

4. $\left|S_{n_{k}}\right|=2^{2 k-3}+2^{k-2}$,

5. $\left|M_{k}\right|=2^{k-1}+2^{k-2}-1$

6. $16 k^{3} \geq\left|P_{k}\right| \geq k^{3}$. 


\section{Capítulo 4}

\section{O grafo de Kř́íz}

\section{Uma construção para grafos com cintura e número cromático grandes sem envolver hipergrafos}

Como foi dito, Erdôs provou a existência de grafos em $\mathcal{G}_{k, g}$ por meios probabilísticos. Assim, a procura de uma demonstração construtiva ainda permanecia. O melhor resultado até então eram os grafos de Tutte, que garantiam cintura pelo menos 6 e número cromático grande. Depois, Nešetřil [30] obteve uma construção para o caso particular em que a cintura é pelo menos 8. A primeira construção para o caso geral de cintura e número cromático foi obtida por Lovász [24] e ela precisava generalizar o problema para hipergrafos. Seguiu-se uma construção mais simples, por Nešetřil e Rödl [33], que também envolvia hipergrafos. Esta construção será vista no Capítulo 5.

Os números de vértices dos grafos obtidos por essas construções eram muito grandes. Possivelmente uma construção que não envolvesse hipergrafos poderia ter menos vértices. Kř́̌ž [23] conseguiu construir grafos em $\mathcal{G}_{k, g}$ sem envolver hipergrafos. Neste capítulo, essa construção é apresentada. Ela será vista primeiro, pois é mais simples que as construções anteriores a ela. Dessa forma, será mais fácil analisar o número de vértices de cada grafo obtido. Não é verdade que esse número é significativamente menor do que os obtidos pelas construções que envolvem hipergrafos.

\subsection{A construção do grafo de Kříž}

Denote por $\mathrm{Kz}_{k, g}$ o grafo de Křřž em $\mathcal{G}_{k, g}$. Esse grafo será obtido de forma indutiva em $k$. Na construção de Tutte, para conseguir $T_{k}$, partia-se de um conjunto $A$ de $(k-1)\left(\left|T_{k-1}\right|-1\right)+1$ vértices independentes e, para cada subconjunto de $A$ de tamanho $\left|T_{k-1}\right|$, era emparelhada uma cópia de $T_{k-1}$. Outra maneira de descrever esse procedimento seria dizer que, a cada um desses subconjuntos de $A$, eram emparelhados $\left|T_{k-1}\right|$ vértices independentes, obtendo-se um grafo base. Depois, em cada um dos conjuntos independentes de tamanho $\left|T_{k-1}\right|$ do grafo base, era inserida uma cópia de $T_{k-1}$. Na construção de $\mathrm{Kz}_{k, g}$, também será tomado um grafo como base, que terá conjuntos independentes de tamanho $\left|\mathrm{Kz}_{k-1, g}\right|$, onde serão inseridas cópias de $\mathrm{Kz}_{k-1, g}$.

Para o argumento de Tutte, era essencial que o conjunto $A$ do grafo base tivesse certo tamanho e fosse constituído de vértices independentes e que cada um dos subconjuntos de tamanho $\left|T_{k-1}\right|$ em $A$ estivesse ligado com outros $\left|T_{k-1}\right|$ vértices por meio de um emparelhamento perfeito. Para o argumento de Kř̌̌ž, também será essencial que o grafo base tenha certas propriedades. Como 
primeiro passo, o grafo base da construção de Křřž é definido e, em seguida, construído.

Uma estrela é uma árvore de diâmetro menor que ou igual a 2. Uma constelação é um grafo tal que toda componente é uma estrela. O grafo base da construção de Křǐž é uma constelação $\Gamma_{i}(n)$, onde os parâmetros inteiros $n \geq 1$ e $i \geq 0$ são tomados de acordo com $k$ e $g$.

A constelação $\Gamma_{i}(n)$ deve satisfazer algumas propriedades. A primeira é que os vértices dessa constelação podem ser particionados em conjuntos independentes de tamanho $n$; chame essa partição de $\mathcal{C}_{i}(n)$. Essa propriedade é importante para a construção de $\mathrm{Kz}_{k, g}$, pois em cada parte será inserida uma cópia de $\mathrm{Kz}_{k-1, g}$, como na construção de Tutte.

A segunda propriedade é importante para garantir número cromático grande. Ela garante que $\mathcal{C}_{i}(n)$ é tal que, tomando um vértice de cada parte, existe necessariamente uma aresta contida nesses vértices. Essa propriedade pode ser reformulada dizendo-se que qualquer conjunto independente da constelação não intersecta alguma das partes.

A terceira propriedade é que garantirá cintura grande. Aqui se define um outro grafo a partir de $\Gamma_{i}(n)$. Em cada uma das partes $X \in \mathcal{C}_{i}(n)$, é inserido um clique $K^{p}(X)$ com $n$ vértices e com um peso $p \geq 0$ qualquer nas arestas. Depois é posto peso 1 nas arestas de $\Gamma_{i}(n)$. O grafo resultante será

$$
\Gamma_{i}^{p}(n)=\Gamma_{i}(n) \cup \bigcup_{X \in \mathcal{C}_{i}(n)} K^{p}(X)
$$

Quando as arestas possuem pesos, o comprimento de um circuito passa a ser a soma dos pesos das arestas dele. Assim, a terceira propriedade garante que o grafo $\Gamma_{i}^{p}(n)$ não contém circuitos de comprimento menor que ou igual a $2^{i}(p+1)$, exceto, possivelmente, dentro de uma parte, isto é, em $K^{p}(X)$ para algum $X \in \mathcal{C}_{i}(n)$.

Definição 4.1.1. Sejam $i \geq 0$ e $n \geq 1$ inteiros. O grafo $\Gamma_{i}(n)$ é uma constelação com as seguintes propriedades.

1. Existe uma partição $\mathcal{C}_{i}(n)$ dos vértices $V\left(\Gamma_{i}(n)\right)$ tal que para todo $X \in \mathcal{C}_{i}(n)$, tem-se $|X|=n$ e $X$ é independente.

2. Para qualquer conjunto independente $M \subset V\left(\Gamma_{i}(n)\right)$, existe $X \in \mathcal{C}_{i}(n)$ tal que $M \cap X=\emptyset$.

3. O grafo com pesos nas arestas $\Gamma_{i}^{p}(n)$ não tem circuitos de comprimento menor que ou igual a $2^{i}(p+1)$ exceto, possivelmente, dentro de uma parte de $\mathcal{C}_{i}(n)$.

Será visto como obter $\mathrm{Kz}_{k, g}$ a partir de um certo $\Gamma_{i}(n)$. Mas, antes, constroem-se os grafos $\Gamma_{i}(n)$.

Lema 4.1.1. Para todo $i \geq 0$ e $n \geq 1$, existe uma constelação $\Gamma_{i}(n)$.

Demonstração. Obtém-se $\Gamma_{i}(n)$ para todo $n$, indutivamente em $i$. As constelações $\Gamma_{0}(n), \operatorname{com} n \geq 1$, são como na Figura 4.1.

Os vértices de $\Gamma_{0}(n)$ estão particionados em $\mathcal{C}_{0}(n)=\left\{X_{0}, \ldots, X_{n}\right\}$. A parte $X_{0}=[n]$ contém os vértices de grau máximo das estrelas de $\Gamma_{0}(n)$; chame esses vértices de centrais. Para $j \geq 1$, a parte $X_{j}=\{(j, 1), \ldots(j, n)\}$ contém os vértices de grau 1 da estrela cujo vértice central é $j$. Assim, $\Gamma_{0}(n)$ é uma constelação e $\mathcal{C}_{0}(n)$ é uma partição de seus vértices em conjuntos independentes de tamanho $n$.

Para checar que $\Gamma_{0}(n)$ satisfaz a propriedade 2 , note que se um conjunto independente contém algum vértice $j \in X_{0}$, então ele não pode conter nenhum vértice de $X_{j}$. Por outro lado, se um 


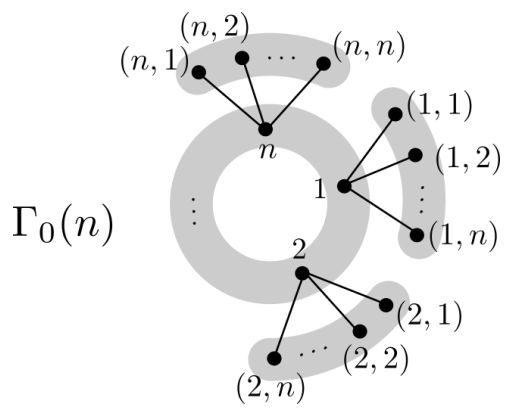

Figura 4.1: A constelação $\Gamma_{0}(n)$ e a partição $\mathcal{C}_{0}(n)$.

conjunto independente é tal que, para todo $j \in[n]$, ele contém um vértice de $X_{j}$, então ele não pode conter nenhum vértice de $X_{0}$. Portanto nenhum conjunto independente em $\Gamma_{0}(n)$ pode intersectar todas as partes de $\mathcal{C}_{0}(n)$.

O grafo $\Gamma_{0}^{p}(n)$ difere de $\Gamma_{0}(n)$, pois as partes de $\mathcal{C}_{0}(n)$ deixam de ser independentes e passam a conter cliques. Além disso, as arestas dos cliques recebem peso $p$ e as arestas de $\Gamma_{0}(n)$ recebem peso 1. Excluindo os circuitos contidos dentro das partes, tem-se que um circuito deve usar uma aresta $j x$ com $j \in X_{0}$ e $x \in X_{j}$, para algum $j \in[n]$. A extremidade $x$ incide apenas em $j$ ou em vértices dentro de $X_{j}$, portanto o circuito deve conter uma aresta $x y$, para algum outro $y \in X_{j}$. Para fechar um circuito, é necessário voltar para a parte $X_{0}$, então é inevitável usar mais uma aresta de $\Gamma_{0}(n)$. Como $y$ também é vizinho de $j$ por uma aresta de $\Gamma_{0}(n)$, tem-se um circuito mínimo $(j, x, y)$ de comprimento $p+2>2^{0}(p+1)$.

Seja $i \geq 0$. Tem-se a seguinte hipótese de indução.

Hipótese 4.1.1.1. A constelação $\Gamma_{i}(n)$ existe para todo $n \geq 1$.

É preciso construir $\Gamma_{i+1}(n)$ para todo $n \geq 1$. Para isso, será feita uma indução em $n$. O grafo $\Gamma_{i+1}(1)$ é definido como uma única aresta $x y$. A partição $\mathcal{C}_{i+1}(1)=\left\{X_{0}, X_{1}\right\}$ tem $X_{0}=\{x\}$ e $X_{1}=\{y\}$. Não é difícil checar que, de fato, $\Gamma_{i+1}(1)$ é uma constelação que satisfaz as propriedades da Definição 4.1.1.

Seja $n \geq 1$. Tem-se a seguinte hipótese de indução.

Hipótese 4.1.1.2. A constelação $\Gamma_{i+1}(n)$ existe.

Para construir $\Gamma_{i+1}(n+1)$, serão unidas cópias de $\Gamma_{i+1}(n)$ e o grafo $\Gamma_{i}\left(\left|\mathcal{C}_{i+1}(n)\right|\right)$.

A ideia é que as partes de $\mathcal{C}_{i+1}(n)$ irão receber, cada uma, um vértice a mais para formar as partes de $\mathcal{C}_{i+1}(n+1)$. Dessa forma, tome $\Gamma_{i+1}(n)$ e adicione $\left|\mathcal{C}_{i+1}(n)\right|$ vértices independentes, um em cada parte de $\mathcal{C}_{i+1}(n)$. Suponha que esse grafo é $\Gamma_{i+1}(n+1)$. Os circuitos mínimos (não contidos numa única parte) em $\Gamma_{i+1}^{p}(n+1)$ e $\Gamma_{i+1}^{p}(n)$ serão os mesmos, uma vez que os vértices novos só se ligam a vértices de sua própria parte, que por sua vez estão diretamente conectados. Assim a propriedade 3 está garantida para $\Gamma_{i+1}(n+1)$. Porém os vértices adicionados não podem ser um conjunto independente, por causa da propriedade 2. Segundo essa propriedade, qualquer conjunto independente de $\Gamma_{i+1}(n+1)$ deve deixar de intersectar alguma parte de $\mathcal{C}_{i+1}(n+1)$ e, portanto, algum dos vértices adicionados.

Uma forma de satisfazer a propriedade 2, sem perder a propriedade 3, é tomar diversas cópias do grafo descrito anteriormente, que é o grafo $\Gamma_{i+1}(n)$ adicionado de vértices independentes, e inserir arestas entre entre alguns dos vértices adicionados, mas de cópias diferentes. Será visto que 
se essas arestas inseridas formarem, junto com os vértices adicionados às cópias de $\Gamma_{i+1}(n)$, uma cópia de $\Gamma_{i}\left(\left|\mathcal{C}_{i+1}(n)\right|\right)$, então o grafo resultante será $\Gamma_{i+1}(n+1)$ (Figura 4.2).

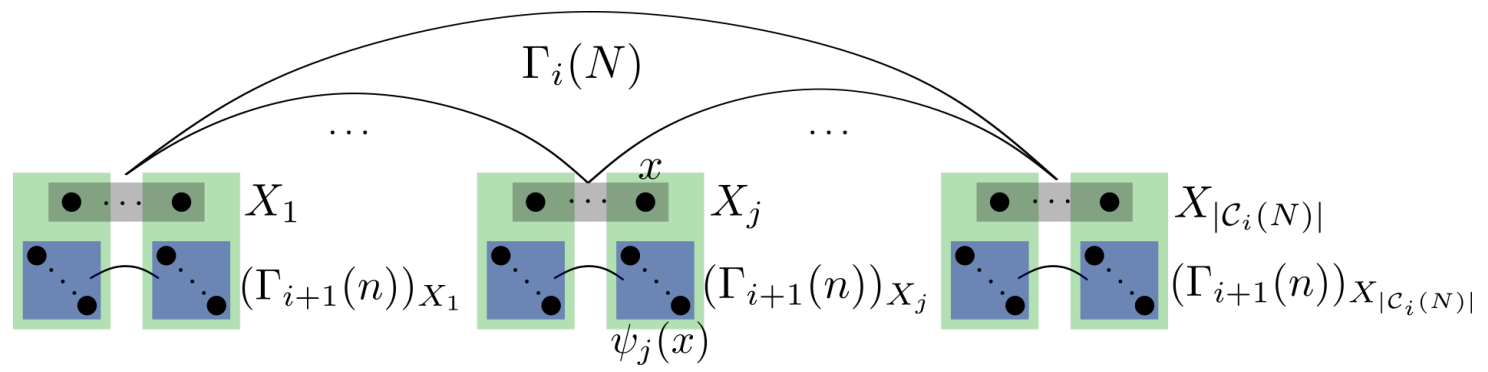

Figura 4.2: $O$ grafo $\Gamma_{i+1}(n+1)$. As partes de $\mathcal{C}_{i+1}(n+1)$ são os retângulos maiores.

Mais precisamente, seja $N=\left|\mathcal{C}_{i+1}(n)\right|$ e tome $\Gamma_{i}(N)$. Para cada parte $X_{j} \in \mathcal{C}_{i}(N)$, tome uma cópia $\left(\Gamma_{i+1}(n)\right)_{X_{j}}$ de $\Gamma_{i+1}(n)$ e seja $\left(\mathcal{C}_{i+1}(n)\right)_{X_{j}}$ a partição dos vértices dessa cópia. A afirmação é que a união disjunta $G=\Gamma_{i}(N) \cup\left(\bigcup_{j \in\left[\left|\mathcal{C}_{i}(N)\right|\right]}\left(\Gamma_{i+1}(n)\right)_{X_{j}}\right)$ será $\Gamma_{i+1}(n+1)$.

De fato, $G$ é uma constelação, pois é uma união disjunta de constelações. Será definida uma partição $\mathcal{C}$ de $V(G)$ que será $\mathcal{C}_{i+1}(n+1)$. Para isso, cada vértice de $X_{j} \in \mathcal{C}_{i}(N)$ será associado a uma parte de $\left(\mathcal{C}_{i+1}(n)\right)_{X_{j}}$. Isso é feito através da bijeção $\psi_{j}: X_{j} \rightarrow\left(\mathcal{C}_{i+1}(n)\right)_{X_{j}}$, note que $\left|X_{j}\right|=N$ e $N=\left|\mathcal{C}_{i+1}(n)\right|$. Assim, tem-se

$$
\mathcal{C}=\bigcup_{j \in\left[\left|\mathcal{C}_{i}(N)\right|\right]}\left\{\{x\} \cup \psi_{j}(x): x \in X_{j}\right\}
$$

Para verificar que $G$ satisfaz a propriedade 2, tome um conjunto independente $M \subset V(G)$. Defina os conjuntos independentes $M_{0}=M \cap V\left(\Gamma_{i}(N)\right)$ e $M_{j}=M \cap V\left(\left(\Gamma_{i+1}(n)\right)_{X_{j}}\right)$ para cada $j \in\left[\left|\mathcal{C}_{i}(N)\right|\right]$. Como $\Gamma_{i}(N)$ e $\Gamma_{i+1}(n)$ satisfazem a propriedade 2 , existem $X_{j_{0}} \in \mathcal{C}_{i}(N)$ tal que $X_{j_{0}} \cap M_{0}=\emptyset$ e $X^{\prime} \in\left(\mathcal{C}_{i+1}(n)\right)_{X_{j_{0}}}$ tal que $X^{\prime} \cap M_{j_{0}}=\emptyset$. Existe um vértice em $X_{j_{0}}$ que foi associado a $X^{\prime}$ para constituir uma parte de $\mathcal{C}$, que é o vértice $x^{\prime}=\psi_{j_{0}}^{-1}\left(X^{\prime}\right)$. Assim, a parte $\left(\left\{x^{\prime}\right\} \cup X^{\prime}\right) \in \mathcal{C}$ não intersecta $M$.

Para verificar que $G$ satisfaz a propriedade 3 , complete as partes de $\mathcal{C}$ com arestas de peso $p \geq 0$ e dê peso 1 para as arestas de $G$; chame esse grafo de $G^{p}$. Serão considerados apenas os circuitos que não estão contidos numa única parte.

Suponha primeiro que qualquer circuito mínimo de $G$ não utiliza nenhuma aresta de $\Gamma_{i}(N)$. Nesse caso, existe um $j_{0} \in\left[\left|\mathcal{C}_{i}(N)\right|\right]$ tal que o circuito está contido no subgrafo $G^{\prime} \subset G^{p}$ induzido por $V\left(\left(\Gamma_{i+1}(n)\right)_{X_{j_{0}}}\right) \cup X_{j_{0}}$. Aqui, é como quando se tentou apenas adicionar vértices independentes em $\Gamma_{i+1}(n)$; tem-se que um circuito mínimo de $G^{\prime}$ e um de $\Gamma_{i+1}^{p}(n)$ têm o mesmo comprimento. Para ver isso, tome um circuito de $G^{\prime}$ que passe por um vértice $x \in X_{j_{0}}$. Em $G^{\prime}$, o vértice $x$ se liga apenas a vértices de $\psi_{j_{0}}(x)$. Como a parte $\{x\} \cup \psi_{j_{0}}(x)$ foi completada com arestas de peso $p$, esse circuito pode ser diminuído ligando-se diretamente os vizinhos de $x$. Assim, um circuito mínimo de $G^{\prime}$ tem vértices apenas em $V\left(\left(\Gamma_{i+1}(n)\right)_{X_{j_{0}}}\right)$, portanto está numa cópia de $\Gamma_{i+1}^{p}(n)$. Essa cópia satisfaz 3, de modo que um circuito mínimo de $G$ tem comprimento maior que $2^{i+1}(p+1)$.

Se algum circuito mínimo $C=\left(x_{1}, \ldots, x_{\ell}\right)$ de $G^{p}$ usa uma aresta de $\Gamma_{i}(N)$, prova-se que ele irá induzir um circuito em $\Gamma_{i}^{2 p+1}(N)$. Sejam $x_{j}$ e $x_{k}$, com $j, k \in[\ell+1], k>j$ e $x_{\ell+1}=x_{1}$, vértices de $C$ que pertencem a $V\left(\Gamma_{i}(N)\right)$ e tais que para todo $x_{m}$ com $j<m<k$, tem-se que $x_{m}$ não pertence a $V\left(\Gamma_{i}(N)\right)$. Se $k=j+1$, então $x_{j}$ e $x_{k}$ estão ligados por uma aresta de $\Gamma_{i}(N)$. Essa 
aresta também está em $\Gamma_{i}^{2 p+1}(N)$ e tem peso 1 , como em $G^{p}$. Se $k>j+1$, existe $j_{0} \in\left[\left|\mathcal{C}_{i}(N)\right|\right]$ tal que $x_{j}, x_{k} \in X_{j_{0}}$. O caminho em $C$ entre $x_{j}$ e $x_{k}$, partindo de $x_{j}$, deve necessariamente entrar em $\psi_{j_{0}}\left(x_{j}\right)$ por uma aresta de peso $p$, usar pelo menos uma aresta de peso 1 para mudar para a parte $\psi_{j_{0}}\left(x_{k}\right)$ e por fim, chegar em $x_{k}$ por outra aresta de peso $p$. Portanto, o caminho tem peso pelo menos $2 p+1$. Em $\Gamma_{i}^{2 p+1}(N)$ existe uma aresta de peso $2 p+1$ entre $x_{j}$ e $x_{k}$, pois a parte $X_{j_{0}}$ foi completada com arestas de peso $2 p+1$. Assim, apagando todos os vértices de $C$ que não pertencem a $V\left(\Gamma_{i}(N)\right)$, é obtido um circuito $C^{\prime}$ em $\Gamma_{i}^{2 p+1}(N)$ de comprimento menor que ou igual ao de $C$. Como $\Gamma_{i}(N)$ satisfaz 3 , o circuito $C^{\prime}$ tem comprimento maior que $2^{i}(2 p+1+1)=2^{i+1}(p+1)$.

Portanto, $G$ e $\mathcal{C}$ satisfazem as propriedades da Definição 4.1.1. Obtem-se que $\Gamma_{i+1}(n+1)=G$ e $\mathcal{C}_{i+1}(n+1)=\mathcal{C}$.

A família de grafos base da construção de Kříž foi construída. O grafo Kz $z_{k, g}$ será obtido indutivamente em $k$. Toma-se o grafo base $\Gamma_{i}\left(\left|\mathrm{Kz}_{k-1, g}\right|\right)$, para certo valor de $i$. Em cada parte de $\mathcal{C}_{i}\left(\left|\mathrm{Kz}_{k-1, g}\right|\right)$, é adicionada uma cópia de $\mathrm{Kz}_{k-1, g}$. Chame o grafo resultante de $\Gamma_{i}\left(\mathrm{Kz}_{k-1, g}\right)$ (Figuras 4.4 e 4.3 ).

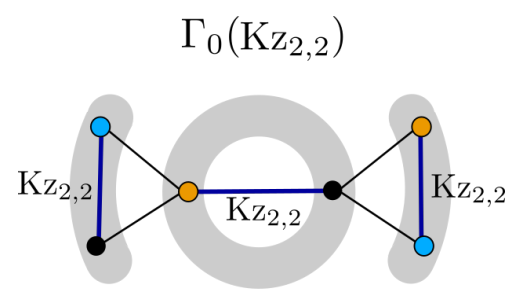

Figura 4.3: $O$ grafo $\mathrm{Kz}_{3,2}$.

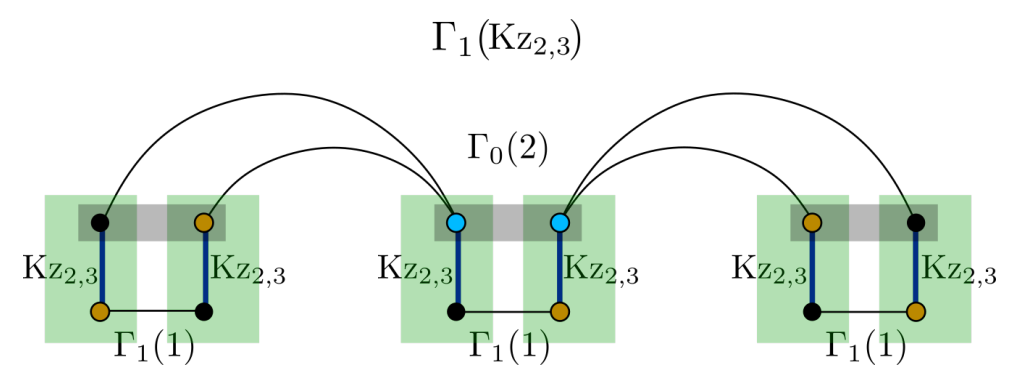

Figura 4.4: $O$ grafo $\mathrm{Kz}_{3,3}$.

Definição 4.1.2. Para $g \geq 2$ e $i=\left\lceil\log _{2}(g / 2)\right\rceil$, defina $\mathrm{Kz}_{2, g}=\Gamma_{i}(1)$. Para $k \geq 3$, defina

$$
\mathrm{Kz}_{k, g}=\Gamma_{i}\left(\mathrm{Kz}_{k-1, g}\right)
$$

Enfim, prova-se que o grafo de Kř̌̌ž tem cintura e número cromático grandes.

Teorema 4.1.2. Para $k \geq 2$ e $g \geq 2$, o grafo $\mathrm{Kz}_{k, g}$ pertence a $\mathcal{G}_{k, g}$.

Demonstração. A prova é uma indução em $k$. Para qualquer $g \geq 2$, seja $i=\left\lceil\log _{2}(g / 2)\right\rceil$, o grafo $\mathrm{Kz}_{2, g}=\Gamma_{i}(1)$ é uma única aresta. Portanto $\chi\left(\mathrm{Kz}_{2, g}\right)=2 \mathrm{e} \mathrm{Kz}_{2, g}$ tem cintura infinita.

Seja $k \geq 2$ e suponha que para todo $g \geq 2$, tem-se que $\mathrm{Kz}_{k, g} \in \mathcal{G}_{k, g}$.

Dado $g \geq 2$ e tomando $i=\left\lceil\log _{2}(g / 2)\right\rceil$, será provado que $\mathrm{Kz}_{k+1, g}=\Gamma_{i}\left(\mathrm{Kz}_{k, g}\right)$ pertence a $\mathcal{G}_{k+1, g}$. Aqui serão utilizadas as propriedades do grafo base. Como notado antes, a propriedade 2 é importante para garantir que o número cromático aumente e 3, para que a cintura permaneça grande. 
Suponha por absurdo que existe uma coloração própria de $\Gamma_{i}\left(\mathrm{Kz}_{k, g}\right) \operatorname{com} k$ cores. Como $\Gamma_{i}\left(\mathrm{Kz}_{k, g}\right)$ é o grafo $\Gamma_{i}\left(\left|\mathrm{Kz}_{k, g}\right|\right)$ acrescentado de algumas arestas, tem-se que $\Gamma_{i}\left(\left|\mathrm{Kz}_{k, g}\right|\right) \subset \Gamma_{i}\left(\mathrm{Kz}_{k, g}\right)$. Assim, o conjunto $M$ dos vértices de $\Gamma_{i}\left(\mathrm{Kz}_{k, g}\right)$ que receberam uma dada cor é um conjunto independente em ambos os grafos. O grafo $\Gamma_{i}\left(\left|\mathrm{Kz}_{k, g}\right|\right)$ satisfaz a propriedade 2, então existe $X \in \mathcal{C}_{i}\left(\left|\mathrm{Kz}_{k, g}\right|\right)$ tal que $M \cap X=\emptyset$. Como $\Gamma_{i}\left(\mathrm{Kz}_{k, g}\right)$ tem uma cópia de $\mathrm{Kz}_{k, g}$ contida na parte $X$, tem-se uma coloração própria de $\mathrm{Kz}_{k, g}$ com $k-1$ cores, uma contradição.

Verifique que, ao inserir cópias de $K z_{k, g}$ nas partes de $\Gamma_{i}\left(\left|\mathrm{~K}_{k, g}\right|\right)$, não foram criados circuitos de comprimento menor que ou igual a $g$. Suponha por absurdo que $\Gamma_{i}\left(\mathrm{Kz}_{k, g}\right)$ contém um circuito $C$ de comprimento no máximo $g$. O grafo com peso 1 nas arestas $\Gamma_{i}^{1}\left(\left|K z_{k, g}\right|\right)$ é obtido a partir de $\Gamma_{i}\left(\left|\mathrm{~K}_{k, g}\right|\right)$, acrescentando-se um clique em cada parte de $\mathcal{C}_{i}\left(\left|\mathrm{Kz} z_{k, g}\right|\right)$. Assim, temse que $\Gamma_{i}\left(\mathrm{Kz}_{k, g}\right) \subset \Gamma_{i}^{1}\left(\left|\mathrm{Kz}_{k, g}\right|\right)$. Dessa forma, o circuito $C$ também está contido em $\Gamma_{i}^{1}\left(\left|\mathrm{Kz}_{k, g}\right|\right)$. Quando as arestas recebem peso 1, a noção de comprimento de um circuito é equivalente à de um grafo sem pesos nas arestas. Tem-se um circuito $C$ em $\Gamma_{i}^{1}\left(\left|\mathrm{Kz}_{k, g}\right|\right)$ de comprimento $g \leq 2^{i+1}$, pela forma que $i$ foi tomado. Pela propriedade 3, excluindo os circuitos contidos numa única parte, o grafo $\Gamma_{i}^{1}\left(\left|\mathrm{Kz}_{k, g}\right|\right)$ não tem circuitos de comprimento menor que $2^{i+1}$. Conclui-se que $C$ está contido em alguma parte $X \in \mathcal{C}_{i}\left(\left|\mathrm{Kz}_{k, g}\right|\right)$. O grafo $\Gamma_{i}\left(\mathrm{~K}_{k, g}\right)$ contém uma cópia de $\mathrm{Kz}_{k, g}$ em $X$. Assim, tem-se que $C$ é um circuito de comprimento no máximo $g$ contido em $K_{k, g}$, uma contradição.

Assim se constroem os grafos de Kř́žz. Como foi dito, não foram utilizados hipergrafos. O próximo passo é verificar que o número de vértices dessa construção é muito grande.

\subsection{O número de vértices do grafo de Kříž}

O grafo $\mathrm{Kz}_{2, g}(g \geq 2)$ é uma aresta, portanto $\left|\mathrm{Kz}_{2, g}\right|=2$. Quando $k \geq 3$, o grafo $\mathrm{Kz}_{k, g}$ é definido como $\Gamma_{i}\left(\mathrm{Kz}_{k-1, g}\right)$, com $i=\left\lceil\log _{2}(g / 2)\right\rceil$. O número de vértices de um grafo $\Gamma_{i}(n)$ é o número de partes $\left|\mathcal{C}_{i}(n)\right|$ vezes $n$. Assim, vale que

$$
\left|\mathrm{Kz}_{k, g}\right|=\left|\mathcal{C}_{i}\left(\left|\mathrm{Kz}_{k-1, g}\right|\right)\right| \cdot\left|\mathrm{Kz}_{k-1, g}\right|
$$

Primeiro serão encontradas uma cota inferior e uma superior para o número de partes $\left|\mathcal{C}_{i}(n)\right|$. Depois essas cotas serão utilizadas na recorrência (4.1), para se encontrar $\left|\mathrm{Kz}_{k, g}\right|$.

Pela forma que a família $\Gamma_{i}(n)(i \geq 0$ e $n \geq 1)$ é construída, tem-se

$$
\left|\mathcal{C}_{i}(n)\right|= \begin{cases}n+1, & i=0 \text { e } n \geq 1 \\ 2, & i>0 \text { e } n=1 \\ \left|\mathcal{C}_{i-1}\left(\left|\mathcal{C}_{i}(n-1)\right|\right)\right| \cdot\left|\mathcal{C}_{i}(n-1)\right|, & i>0 \text { e } n>1\end{cases}
$$

Primeiro é apresentada uma cota inferior.

Proposição 4.2.1. Dados inteiros $i \geq 1$ e $n \geq 1$, vale que $\left|\mathcal{C}_{i}(n)\right| \geq 2 \uparrow^{i} n$.

Demonstração. É feita uma indução em $i$. Quando $i=1$, prova-se que $\left|\mathcal{C}_{1}(n)\right| \geq 2^{2^{n-1}}$ por indução em $n$. Tem-se que $\left|\mathcal{C}_{1}(1)\right|=2=2^{2^{0}}$. Seja $n \geq 2$ e suponha que $\left|\mathcal{C}_{1}(n-1)\right| \geq 2^{2^{n-2}}$. 
Como $\left|\mathcal{C}_{i}(n)\right|$ é dado por (4.2), vale que

$$
\begin{aligned}
\left|\mathcal{C}_{1}(n)\right| & =\left|\mathcal{C}_{0}\left(\left|\mathcal{C}_{1}(n-1)\right|\right)\right| \cdot\left|\mathcal{C}_{1}(n-1)\right| \\
& =\left(\left|\mathcal{C}_{1}(n-1)\right|+1\right) \cdot\left|\mathcal{C}_{1}(n-1)\right| \\
& \geq\left|\mathcal{C}_{1}(n-1)\right|^{2} \geq\left(2^{2^{n-2}}\right)^{2}=2^{2^{n-1}}
\end{aligned}
$$

Como $2^{2^{n-1}} \geq 2^{n}(n \geq 1)$ e $2^{n}=2 \uparrow n$, tem-se $\left|\mathcal{C}_{1}(n)\right| \geq 2 \uparrow n$.

Agora seja $i \geq 2$. Suponha que, para todo $n \geq 1$, tem-se $\left|\mathcal{C}_{i-1}(n)\right| \geq 2 \uparrow^{i-1} n$.

Será feita uma indução em $n$ para provar que $\left|\mathcal{C}_{i}(n)\right| \geq 2 \uparrow^{i} n$. Tem-se que $\left|\mathcal{C}_{i}(1)\right|=2=2 \uparrow^{i} 1$. Seja $n \geq 2$ e suponha que $\left|\mathcal{C}_{i}(n-1)\right| \geq 2 \uparrow^{i}(n-1)$.

Mais uma vez usando (4.2), vale que

$$
\begin{aligned}
\left|\mathcal{C}_{i}(n)\right| & =\left|\mathcal{C}_{i-1}\left(\left|\mathcal{C}_{i}(n-1)\right|\right)\right| \cdot\left|\mathcal{C}_{i}(n-1)\right| \\
& \geq 2 \uparrow^{i-1}\left(2 \uparrow^{i}(n-1)\right) \cdot 2 \uparrow^{i}(n-1) \\
& \geq 2 \uparrow^{i-1}\left(2 \uparrow^{i}(n-1)\right)=2 \uparrow^{i} n .
\end{aligned}
$$

Para a cota superior do número de partes, o procedimento será parecido.

Proposição 4.2.2. Dados inteiros $i \geq 1$ e $n \geq 1$, tem-se $\left|\mathcal{C}_{i}(n)\right| \leq 2 \uparrow^{i}\left(2^{n}+2 n\right)$.

Demonstração. É feita uma indução em $i$. Quando $i=1$, prova-se que $\mathcal{C}_{1}(n) \leq 2^{2^{n}-1}$ por indução em $n$. Tem-se que $\left|\mathcal{C}_{1}(1)\right|=2=2^{2^{1}-1}$.

Seja $n \geq 2$ e suponha que $\left|\mathcal{C}_{1}(n-1)\right| \leq 2^{2^{n-1}-1}$.

Usando (4.2), vale que

$$
\begin{aligned}
\left|\mathcal{C}_{1}(n)\right| & =\left|\mathcal{C}_{0}\left(\left|\mathcal{C}_{1}(n-1)\right|\right)\right| \cdot\left|\mathcal{C}_{1}(n-1)\right| \\
& =\left(\left|\mathcal{C}_{1}(n-1)\right|+1\right) \cdot\left|\mathcal{C}_{1}(n-1)\right| \\
& \leq 2 \cdot\left|\mathcal{C}_{1}(n-1)\right|^{2} \leq 2 \cdot\left(2^{2^{n-1}-1}\right)^{2}=2^{2^{n}-1}
\end{aligned}
$$

Como $2^{2^{n}-1} \leq 2^{2^{n}+2 n}$ e uma flecha equivale a exponenciação, tem-se $\left|\mathcal{C}_{1}(n)\right| \leq 2 \uparrow\left(2^{n}+2 n\right)$.

Agora seja $i \geq 2$. Suponha que, para todo $n \geq 1$, tem-se $\left|\mathcal{C}_{i-1}(n)\right| \leq 2 \uparrow^{i-1}\left(2^{n}+2 n\right)$.

Será feita uma indução em $n$ para provar que $\left|\mathcal{C}_{i}(n)\right| \leq 2 \uparrow^{i}\left(2^{n}+2 n\right)$. Para o caso base, vale que $\left|\mathcal{C}_{i}(1)\right|=2=2 \uparrow^{i} 1 \leq 2 \uparrow^{i}\left(2^{1}+2 \cdot 1\right)$.

Seja $n \geq 2$ e suponha que $\left|\mathcal{C}_{i}(n-1)\right| \leq 2 \uparrow^{i}\left(2^{n-1}+2(n-1)\right)$.

Mais uma vez usando (4.2), vale que

$$
\begin{aligned}
\left|\mathcal{C}_{i}(n)\right| & =\left|\mathcal{C}_{i-1}\left(\left|\mathcal{C}_{i}(n-1)\right|\right)\right| \cdot\left|\mathcal{C}_{i}(n-1)\right| \\
& \leq 2 \uparrow^{i-1}\left(2 \uparrow\left|\mathcal{C}_{i}(n-1)\right|+2\left|\mathcal{C}_{i}(n-1)\right|\right) \cdot 2 \uparrow^{i}\left(2^{n-1}+2(n-1)\right) .
\end{aligned}
$$

Use a hipótese de indução no termo $2 \uparrow\left|\mathcal{C}_{i}(n-1)\right|$. Tem-se

$$
\begin{aligned}
2 \uparrow\left|\mathcal{C}_{i}(n-1)\right| & \leq 2 \uparrow\left(2 \uparrow^{i}\left(2^{n-1}+2(n-1)\right)\right) \\
& \leq 2 \uparrow^{i-1}\left(2 \uparrow^{i}\left(2^{n-1}+2(n-1)\right)\right)=2 \uparrow^{i}\left(2^{n-1}+2(n-1)+1\right),
\end{aligned}
$$


onde a segunda desigualdade vale, pois $i \geq 2$.

Também usando a hipótese de indução, o termo $2\left|\mathcal{C}_{i}(n-1)\right|$ fica

$$
\begin{aligned}
2\left|\mathcal{C}_{i}(n-1)\right| & \leq 2 \cdot 2 \uparrow^{i}\left(2^{n-1}+2(n-1)\right) \\
& \leq 2 \uparrow\left(2 \uparrow^{i}\left(2^{n-1}+2(n-1)\right)\right) \\
& \leq 2 \uparrow^{i-1}\left(2 \uparrow^{i}\left(2^{n-1}+2(n-1)\right)\right)=2 \uparrow^{i}\left(2^{n-1}+2(n-1)+1\right) .
\end{aligned}
$$

Assim,

$$
\begin{aligned}
2 \uparrow^{i-1}\left(2 \uparrow\left|\mathcal{C}_{i}(n-1)\right|+2\left|\mathcal{C}_{i}(n-1)\right|\right) & \leq 2 \uparrow^{i-1}\left(2 \cdot 2 \uparrow^{i}\left(2^{n-1}+2(n-1)+1\right)\right) \\
& \leq 2 \uparrow^{i-1}\left(2 \uparrow^{i}\left(2^{n-1}+2(n-1)+2\right)\right) \\
& \leq 2 \uparrow^{i}\left(2^{n-1}+2(n-1)+3\right) .
\end{aligned}
$$

Por fim, completa-se a cota superior para $\left|\mathcal{C}_{i}(n)\right|$.

$$
\begin{aligned}
\left|\mathcal{C}_{i}(n)\right| & \leq 2 \uparrow^{i}\left(2^{n-1}+2(n-1)+3\right) \cdot 2 \uparrow^{i}\left(2^{n-1}+2(n-1)\right) \\
& \leq\left(2 \uparrow^{i}\left(2^{n-1}+2(n-1)+3\right)\right)^{2} \\
& \leq 2 \uparrow^{i}\left(2^{n-1}+2(n-1)+4\right) \\
& =2 \uparrow^{i}\left(2^{n-1}+2 n+2\right) \leq 2 \uparrow^{i}\left(2^{n}+2 n\right),
\end{aligned}
$$

onde a última desigualdade vale, pois $2 \leq 2^{n-1}(n \geq 2)$.

Para encontrar uma cota inferior do número de vértices de $\mathrm{Kz}_{k, g}$, utiliza-se a recorrência (4.1) e a cota do número de partes dada pela Proposição 4.2.1.

Teorema 4.2.3. Sejam inteiros $k \geq 3$ e $g \geq 3$. Se $i=\left\lceil\log _{2}(g / 2)\right\rceil$, então $\left|\mathrm{Kz}_{k, g}\right| \geq 2 \uparrow^{i+1}(k-1)$. Demonstração. Se $g \geq 3$, então $i=\left\lceil\log _{2}(g / 2)\right\rceil \geq 1$. Se $k \geq 3$, então $\left|\mathrm{Kz}_{k-1, g}\right| \geq\left|\mathrm{Kz}_{2, g}\right|=2$. Assim, pode-se utilizar a Proposição 4.2.1 na recorrência (4.1), obtém-se

$$
\left|\mathrm{Kz}_{k, g}\right| \geq\left|\mathrm{Kz}_{k-1, g}\right| \cdot 2 \uparrow^{i}\left|\mathrm{Kz}_{k-1, g}\right|
$$

Será feita uma indução em $k$, para todo $g \geq 3$. Quando $k=3$, vale que

$$
\left|\mathrm{Kz}_{3, g}\right| \geq 2 \uparrow^{i}\left|\mathrm{Kz}_{2, g}\right|=2 \uparrow^{i} 2=2 \uparrow^{i+1} 2 .
$$

A última igualdade vale, pois $2 \uparrow^{i+1} 2=2 \uparrow^{i}\left(2 \uparrow^{i+1} 1\right)=2 \uparrow^{i} 2$.

Seja $k \geq 4$ e suponha que $\left|\mathrm{Kz}_{k-1, g}\right| \geq 2 \uparrow^{i+1}(k-2)$ para qualquer $g \geq 3$.

Assim, vale que

$$
\begin{aligned}
\left|\mathrm{Kz}_{k, g}\right| & \geq 2 \uparrow^{i}\left|\mathrm{Kz}_{k-1, g}\right| \\
& \geq 2 \uparrow^{i} 2 \uparrow^{i+1}(k-2)=2 \uparrow^{i+1}(k-1) .
\end{aligned}
$$

Encontrar uma cota superior de $\left|\mathrm{Kz}_{k, g}\right|$ será parecido. Utiliza-se a recorrência (4.1) e a cota do número de partes dada pela Proposição 4.2.2. 
Teorema 4.2.4. Sejam inteiros $k \geq 4$ e $g \geq 5$. Se $i=\left\lceil\log _{2}(g / 2)\right\rceil$, então $\left|\mathrm{Kz}_{k, g}\right| \leq 2 \uparrow^{i+1}(k+8)$. Demonstração. Como antes, pode-se utilizar a Proposição 4.2 .2 na recorrência (4.1). Obtém-se

$$
\begin{aligned}
\left|\mathrm{Kz}_{k, g}\right| & \leq\left|\mathrm{Kz}_{k-1, g}\right| \cdot 2 \uparrow^{i}\left(2^{\left|\mathrm{Kz}_{k-1, g}\right|}+2\left|\mathrm{Kz}_{k-1, g}\right|\right) \\
& \leq\left(2 \uparrow^{i}\left(2^{\left|\mathrm{Kz}_{k-1, g}\right|}+2\left|\mathrm{Kz}_{k-1, g}\right|\right)\right)^{2},
\end{aligned}
$$

onde também foi usado que $2 \uparrow^{i}\left(2^{\left|\mathrm{Kz}_{k-1, g}\right|}+2\left|\mathrm{~K} z_{k-1, g}\right|\right) \geq 2 \uparrow^{0} 2^{\left|\mathrm{Kz}_{k-1, g}\right|} \geq\left|\mathrm{Kz}_{k-1, g}\right|$.

Para lidar com $\left(2 \uparrow^{i}\left(2^{\left|\mathrm{Kz}_{k-1, g}\right|}+2\left|\mathrm{Kz}_{k-1, g}\right|\right)\right)^{2}$, será provado que $\left(2 \uparrow^{i} x\right)^{2} \leq 2 \uparrow^{i}(x+1)$ para todo $i \geq 2$ e $x \geq 0$. Para isso, use que $a^{2} \leq 2^{a}$ para todo $a \geq 0$ e $a \neq 3$. Tem-se que $\left(2 \uparrow^{i} x\right) \neq 3$, pois vale que $2 \uparrow^{i} 0=1$, que $2 \uparrow^{i} 1=2$ e que $2 \uparrow^{i} x \geq 4$ para $x \geq 2$. Assim,

$$
\left(2 \uparrow^{i} x\right)^{2} \leq 2 \uparrow\left(2 \uparrow^{i} x\right) \leq 2 \uparrow^{i-1}\left(2 \uparrow^{i} x\right)=2 \uparrow^{i}(x+1),
$$

onde a segunda desigualdade vale se $i \geq 2$.

Como $i=\left\lceil\log _{2}(g / 2)\right\rceil \geq 2(g \geq 5)$, pode-se escrever

$$
\begin{aligned}
\left|\mathrm{Kz}_{k, g}\right| & \leq 2 \uparrow^{i}\left(2^{\left|\mathrm{Kz}_{k-1, g}\right|}+2\left|\mathrm{Kz}_{k-1, g}\right|+1\right) \\
& \leq 2 \uparrow^{i} 2^{2\left|\mathrm{Kz}_{k-1, g}\right|}
\end{aligned}
$$

onde a segunda desigualdade vale, pois $2^{x}+2 x+1 \leq 2^{2 x}$ para $x \geq 2$ e $\left|\mathrm{Kz}_{k-1, g}\right| \geq 2$ para $k \geq 3$.

Agora será provado por indução em $k$ que para todo $k \geq 3$ e $g \geq 5$, tem-se

$$
2^{2\left|\mathrm{Kz}_{k, g}\right|} \leq \underbrace{2 \uparrow^{i} \ldots 2 \uparrow^{i}}_{k-2} 11 .
$$

O caso base é $2^{2\left|\mathrm{Kz}_{3, g}\right|}=2 \uparrow 2 \uparrow^{0}\left|\mathrm{Kz}_{3, g}\right|$. Usando (4.3), tem-se

$$
2^{2\left|\mathrm{Kz}_{3, g}\right|} \leq 2 \uparrow 2 \uparrow^{0}\left(2 \uparrow^{i}\left(2^{\left|\mathrm{Kz}_{2, g}\right|}+2\left|\mathrm{Kz}_{2, g}\right|+1\right)\right) \leq 2 \uparrow^{i} 11 .
$$

Seja $k \geq 4$ e suponha que $2^{2\left|\mathrm{Kz}_{k-1, g}\right|} \leq \underbrace{2 \uparrow^{i} \ldots 2 \uparrow^{i}}_{k-3} 11$ para todo $g \geq 5$.

Usando (4.3), tem-se

$$
\begin{aligned}
2^{2\left|\mathrm{Kz}_{k, g}\right|} & \leq 2 \uparrow 2 \uparrow^{0}\left(2 \uparrow^{i}\left(2^{\left|\mathrm{Kz}_{k-1, g}\right|}+2\left|\mathrm{Kz}_{k-1, g}\right|+1\right)\right) \\
& \leq 2 \uparrow^{i}\left(2^{\left|\mathrm{Kz}_{k-1, g}\right|}+2\left|\mathrm{Kz}_{k-1, g}\right|+3\right) \\
& \leq 2 \uparrow^{i}\left(2^{2\left|\mathrm{Kz}_{k-1, g}\right|}\right)
\end{aligned}
$$

onde a terceira desigualdade vale, pois $2^{x}+2 x+3 \leq 2^{2 x}$ para $x \geq 2$.

Usando a hipótese de indução, tem-se

$$
\begin{aligned}
2^{2\left|\mathrm{Kz}_{k, g}\right|} & \leq 2 \uparrow^{i}\left(2^{2\left|\mathrm{Kz}_{k-1, g}\right|}\right) \\
& \leq 2 \uparrow^{i}(\underbrace{2 \uparrow^{i} \ldots 2 \uparrow^{i}}_{k-3} 11)=\underbrace{2 \uparrow^{i} \ldots 2 \uparrow^{i}}_{k-2} 11 .
\end{aligned}
$$


Enfim, utiliza-se esse resultado em (4.4). Para $k \geq 4$, tem-se

$$
\begin{aligned}
\left|\mathrm{Kz}_{k, g}\right| & \leq 2 \uparrow^{i} 2^{2\left|\mathrm{Kz}_{k-1, g}\right|} \leq 2 \uparrow^{i}(\underbrace{2 \uparrow^{i} \ldots 2 \uparrow^{i}}_{k-3} 11) \\
& \leq \underbrace{2 \uparrow^{i} \ldots 2 \uparrow^{i}}_{k-3}\left(2 \uparrow^{i+1} 11\right) \leq 2 \uparrow^{i+1}(k+8) .
\end{aligned}
$$

Portanto, como foi antecipado, o número de vértices da construção de Křřž é Ackermanniano. O tamanho dessa construção, no entanto, é menor que o da construção por amalgamação. Isso será visto no próximo capítulo. 


\section{Capítulo 5}

\section{O Amálgama}

\section{Combinando grafos com hipergrafos para construir um grafo de cintura e número cromático grandes}

Neste capítulo, será vista a construção de Nešetřil e Rödl [33]. Essa construção, que antecede a de Křřž, conseguiu resolver o problema de se construir um grafo $\mathcal{A}_{2, k, g} \in \mathcal{G}_{k, g}$ utilizando-se de hipergrafos. Às vezes, resolver um problema mais geral é mais imediato que resolver um caso particular dele. Os hipergrafos que serão construídos para se conseguir $\mathcal{A}_{2, k, g}$ são uma junção de dois outros hipergrafos, por isso se chamam amálgamas.

No que segue, apresenta-se como é feita a amalgamação de dois hipergrafos. Depois, é visto como utilizar amálgamas para se construir $\mathcal{A}_{2, k, g}$. Por fim, conta-se o número de vértices dessa construção.

\subsection{A amalgamação}

No Capítulo 4, foi dito que a construção de Křriž pode ser relacionada à de Tutte, porque cópias disjuntas de $\mathrm{Kz}_{k-1, g}$ são inseridas num grafo auxiliar para se obter $\mathrm{Kz}_{k, g}$. Na construção de Tutte, o conjunto de vértices independentes $A$ mais as arestas do emparelhamento seriam o grafo auxiliar. Em ambas construções, a conexão entre as diferentes cópias do grafo em $\mathcal{G}_{k-1, g}$ já obtido é feita através do grafo auxiliar, obtendo-se um grafo em $\mathcal{G}_{k, g}$.

A amalgamação de hipergrafos também pode ser relacionada à construção de Tutte, porém através uma interpretação diferente. Na construção de Tutte, para se conseguir $T_{k}$, toma-se um conjunto $A$ formado por um certo número de vértices independentes. Depois, para cada subconjunto de $A$ de tamanho $\left|T_{k-1}\right|$, toma-se uma cópia de $T_{k-1}$ e seus vértices são emparelhados com os do subconjunto. O conjunto $A$, dessa vez, pode ser visto como os vértices de um hipergrafo $\mathcal{A}$, que é $\left|T_{k-1}\right|$-uniforme e completo. As cópias de $T_{k-1}$ são associadas a cada uma das arestas de $\mathcal{A}$ por um emparelhamento perfeito, continuando disjuntas entre si. Porém os emparelhamentos entre cópias de $T_{k-1}$ e arestas de $\mathcal{A}$ acabam relacionando as cópias disjuntas de $T_{k-1}$, pois as arestas de $\mathcal{A}$ podem se intersectar. É como se as cópias tivessem sido de certa forma "costuradas" uma a outra, adicionando-se alguns vértices.

Também na amalgamação entre os hipergrafos $\mathcal{X}$ e $\mathcal{Y}$, são as arestas de $\mathcal{Y}$ que determinarão como serão costuradas cópias disjuntas de $\mathcal{X}$. Porém não serão adicionados novos vértices. O hipergrafo $\mathcal{X}$ 
será $a$-partido, o que significa que seus vértices podem ser particionados em conjuntos independentes. A conexão entre cópias de $\mathcal{X}$ será feita através da identificação de vértices de cópias distintas que estejam em partes correspondentes. Quais vértices serão identificados fica determinado pela forma que arestas de $\mathcal{Y}$ se intersectam. Para isso, os hipergrafos $\mathcal{X}$ e $\mathcal{Y}$ devem ter formas compatíveis, assim como as arestas de $\mathcal{A}$ precisavam ter $\left|T_{k-1}\right|$ vértices. A amalgamação é descrita na Definição 5.1.2.

Definição 5.1.1. Dados inteiros $a$ e $p$, com $a \geq p \geq 2$, um $p$-grafo $(X, \mathcal{M})$ é dito a-partido quando $X=\bigcup_{i=1}^{a} X_{i}$, onde $X_{i}$ são dois a dois disjuntos e não vazios e, para qualquer aresta $M \in \mathcal{M}$ e $i \in[a]$, vale que $\left|M \cap X_{i}\right| \leq 1$.

Se $\mathcal{X}=(X, \mathcal{M})$ é um $p$-grafo $a$-partido, então $X=\bigcup_{i=1}^{a} X_{i}$. Tome $\mathcal{Y}=(Y, \mathcal{N})$ um $\left|X_{r}\right|$-grafo para algum $r \in[a]$. O tamanho das arestas de $\mathcal{Y}$ é igual ao tamanho da parte $X_{r}$ de $\mathcal{X}$. É através de $X_{r}$ que as cópias de $\mathcal{X}$ serão costuradas; elas serão disjuntas a menos da parte $X_{r}$.

O amálgama entre $\mathcal{X}$ e $\mathcal{Y}$ será denotado $\mathcal{Y} * \mathcal{X}$. O amálgama também será $p$-uniforme $a$-partido. Para obtê-lo, toma-se o hipergrafo $\mathcal{Y}$ e, para cada aresta de $\mathcal{N}$, é unida uma cópia de $\mathcal{X}$. A união de cada cópia é disjunta a menos da parte $X_{r}$, essa parte é identificada com a aresta de $\mathcal{N}$ correspondente à cópia em questão (Figura 5.1).

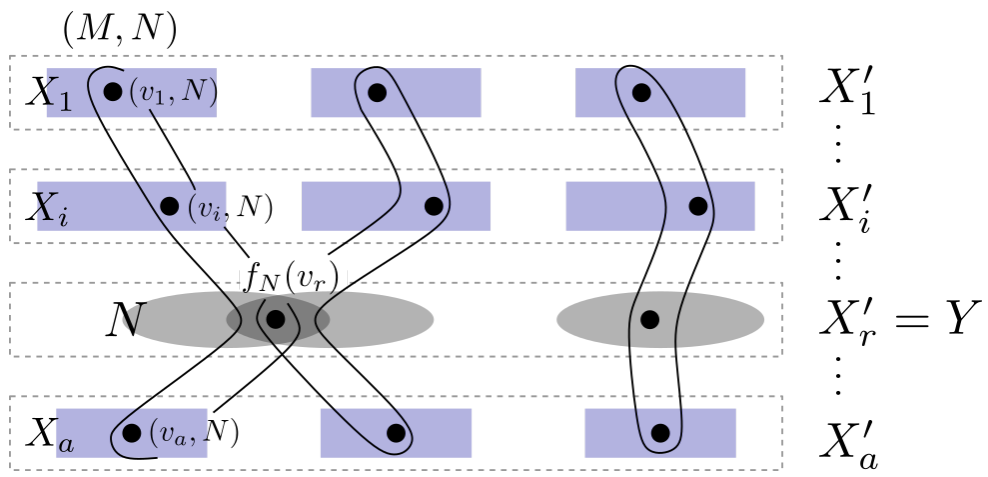

Figura 5.1: $O$ amálgama $\mathcal{Y} * \mathcal{X}$.

Define-se mais formalmente o amálgama.

Definição 5.1.2. Sejam $\mathcal{X}=(X, \mathcal{M})$, com $X=\bigcup_{i=1}^{a} X_{i}$, um $p$-grafo $a$-partido e $\mathcal{Y}=(Y, \mathcal{N})$ um $\left|X_{r}\right|$-grafo, para algum $r \in[a]$. Seja dada, para toda aresta $N \in \mathcal{N}$, uma bijeção $f_{N}: X_{r} \rightarrow N$. O amálgama $\mathcal{Y} * \mathcal{X}=\left(X^{\prime}, \mathcal{M}^{\prime}\right)$ é um p-grafo $a$-partido com $X^{\prime}=\bigcup_{i=1}^{a} X_{i}^{\prime}$. Para $i \in[a] \backslash r$, temse $X_{i}^{\prime}=\left\{(v, N): v \in X_{i}\right.$ e $\left.N \in \mathcal{N}\right\}$ e $X_{r}^{\prime}=Y$. As arestas são $\mathcal{M}^{\prime}=\{(M, N): M \in \mathcal{M}$ e $N \in \mathcal{N}\}$, onde $(M, N)=\left\{(v, N): v \in\left(M \cap X_{i}\right), i \in[a] \backslash r\right\} \cup\left\{f_{N}\left(M \cap X_{r}\right)\right\}$.

Assim, cada vértice de $X^{\prime}$ é uma cópia de algum vértice $v \in X$. Para cada $N \in \mathcal{N}$, existe uma cópia de $v$, que é $(v, N)$, exceto se $v \in X_{r}$, quando a cópia é $f_{N}(v)$. As arestas de $\mathcal{M}^{\prime}$ são tomadas de forma correspondente às arestas de $\mathcal{M}$, usando cópias de vértices que correspondam a um mesmo $N \in \mathcal{N}$. Para cada $N \in \mathcal{N}$, existe uma cópia de $M \in \mathcal{M}$, que é $(M, N)$. A aresta $(M, N)$ contém as cópias dos vértices de $M$ com segunda coordenada $N$ e, se houver vértice $v \in M \cap X_{r}$, ela conterá a cópia $f_{N}(v)$. 


\subsection{A construção de $\mathcal{A}_{p, k, g}$}

O grafo de Kř́̌̌z em $\mathcal{G}_{k, g}$ é da seguinte forma

$$
\mathrm{Kz}_{k, g}=\underbrace{\Gamma_{i}\left(\ldots\left(\Gamma_{i}\left(\Gamma_{i}(1)\right)\right) \ldots\right)}_{k-2}
$$

onde $i=\left\lceil\log _{2}(g / 2)\right\rceil$. Ele é obtido indutivamente em $k$ e através da repetição de um dado procedimento, que é inserir uma cópia do grafo recém obtido em cada parte de um $\Gamma_{i}(n)$ apropriado. Em cada etapa, obtém-se um $\mathrm{Kz}_{k^{\prime}, g}$ com $k^{\prime}<k$. No final, o grafo de Kř̌́ž contém várias cópias de cada constelação utilizada e também de cada $\mathrm{Kz}_{k^{\prime}, g} \operatorname{com} k^{\prime}<k$.

O hipergrafo $\mathcal{A}_{p, k, g}$ também será obtido indutivamente, porém em $g$, para todo $p \geq 2$ e $k \geq 2$. Partindo-se de um certo hipergrafo $\mathcal{X}^{1}$, serão feitas diversas amalgamações envolvendo o hipergrafo recém obtido e outro dado por indução. No final, o grafo obtido será $\mathcal{A}_{p, k, g}$ e ele irá conter várias cópias de cada um dos hipergrafos intermediários que foram obtidos, porém não dos hipergrafos dados por hipótese de indução, dos quais conterá apenas cópias de seus vértices.

O hipergrafo inicial $\mathcal{X}^{1}$ deve ter algumas propriedades. De modo que, assim como a família $\Gamma_{i}(n)$ satisfazia certas propriedades, os hipergrafos obtidos a cada amalgamação também irão manter certas propriedades. Assim, tem-se que $\mathcal{X}^{1}$ é um $p$-grafo $a$-partido, para que se possa fazer a amalgamação, e $a$ depende de $k$ e $p$. Ele também satisfaz outras duas propriedades, que servirão uma para garantir a cintura grande e outra para o número cromático grande. Isso será visto adiante.

Tendo $\mathcal{X}^{1}$, o que é feito em seguida se assemelha a inserir uma cópia de $\mathrm{Kz}_{k-1, g}$ em cada parte de $\Gamma_{i}(n)$ na construção de Kř́žz. Com a diferença que serão tomadas diversas cópias de $\mathcal{X}^{1}$ e não do hipergrafo dado por indução. Serão feitas a amalgamações. A primeira é $\mathcal{A}_{p_{1}, k, g-1} * \mathcal{X}^{1}$, onde $\mathcal{A}_{p_{1}, k, g-1}$ é o hipergrafo dado por indução, com $p_{1}$ sendo o tamanho de alguma parte de $\mathcal{X}^{1}$. Pela definição de amalgamação, essa parte será substituída pelos vértices de $\mathcal{A}_{p_{1}, k, g-1}$ no amálgama resultante e ele irá conter várias cópias de $\mathcal{X}^{1}$. Para $2 \leq t \leq a+1$, chame o hipergrafo obtido pela $(t-1)$-ésima amalgamação de $\mathcal{X}^{t}$. A $t$-ésima amalgamação é $\mathcal{A}_{p_{t}, k, g-1} * \mathcal{X}^{t}$, onde $p_{t}$ é o tamanho de uma parte de $\mathcal{X}^{t}$ que não contenha vértices de nenhum dos $\mathcal{A}_{p_{t^{\prime}}, k, g-1}\left(t^{\prime}<t\right)$ usados nas amalgamações anteriores (Figura 5.2). Aplicar a amalgamação a todas as partes irá garantir que o último grafo obtido tenha número cromático pelo menos $k$. E usar hipergrafos $\mathcal{A}_{p_{t}, k, g-1}$ dados pela hipótese de indução garantirá que as amalgamações não gerem circuitos de comprimento no máximo $g$. Assim, o hipergrafo obtido pela $a$-ésima amalgamação, $\mathcal{X}^{a+1}$, será um hipergrafo em $\mathcal{G}_{p, k, g}$.

Teorema 5.2.1. Para quaisquer inteiros $p \geq 2, k \geq 2$ e $g \geq 1$, existe p-grafo a-partido $\mathcal{A}_{p, k, g} \in \mathcal{G}_{p, k, g}$, onde $a=(k-1)(p-1)+1 \geq p$.

Demonstração. A prova é feita por indução em $g$. Para $g=1$, não há restrição sobre a cintura do hipergrafo. Assim, para todo $p \geq 2$ e $k \geq 2$, defina $\mathcal{A}_{p, k, 1}$ como o $p$-grafo completo com $(k-1)(p-1)+1$ vértices. Neste caso, $\mathcal{A}_{p, k, 1}$ é $a$-partido e cada parte é um único vértice. Pelo princípio da casa dos pombos, qualquer partição em $k-1$ cores terá uma cor que ocorre em pelo menos $p$ vértices e, como o grafo é completo, tem-se uma aresta monocromática.

Seja $g \geq 2$ e suponha que se tem um $p$-grafo $a$-partido $\mathcal{A}_{p, k, g-1} \in \mathcal{G}_{p, k, g-1}$ para quaisquer $k \geq 2$ e $p \geq 2$. Será construído $\mathcal{A}_{p, k, g} \in \mathcal{G}_{p, k, g}$. Para isso, dados $k \geq 2$ e $p \geq 2$, é construída uma sequência $\left(\mathcal{X}^{1}, \ldots, \mathcal{X}^{a+1}\right)$ de $p$-grafos $a$-partidos e $\mathcal{A}_{p, k, g}$ será $\mathcal{X}^{a+1}$. 


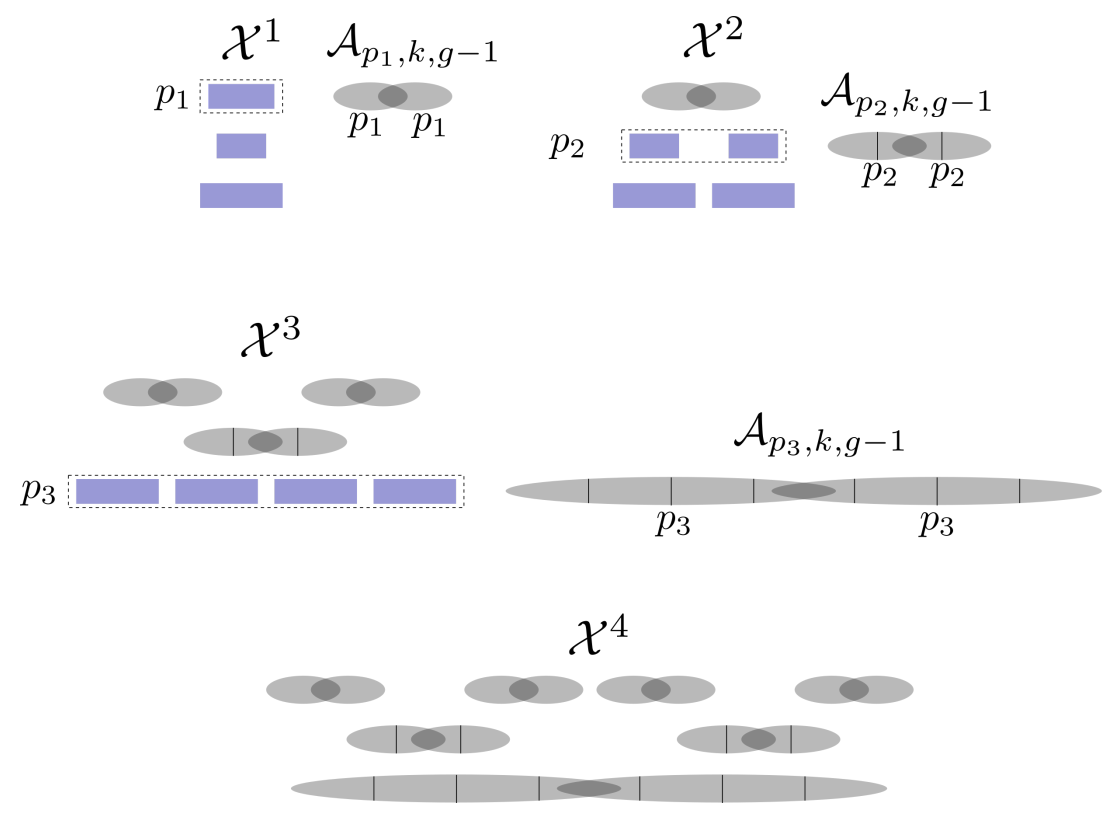

Figura 5.2: Construção da sequência $\left(\mathcal{X}^{1}, \ldots, \mathcal{X}^{4}\right)$.

O hipergrafo $\mathcal{X}^{1}=\left(X^{1}, \mathcal{M}^{1}\right)$ deve ter duas propriedades. A primeira é que ele não contém circuitos. A segunda é que, para qualquer escolha de $p$ partes, existirá uma aresta contida nela. Isto é, se $X_{i}^{1}(1 \leq i \leq a)$ são as partes de $X^{1}$, então para qualquer $\omega \subset[a] \operatorname{com}|\omega|=p$, existe $M \in \mathcal{M}^{1}$ tal que $M \cap X_{i}^{1} \neq \emptyset(i \in \omega)$. Um tal hipergrafo $\mathcal{X}^{1}$ existe, basta tomar uma aresta disjunta para cada escolha de $p$ partes.

Suponha que se tem a sequência até $\mathcal{X}^{t}=\left(X^{t}, \mathcal{M}^{t}\right)$ e que as partes de $X^{t}$ são $X_{i}^{t}(1 \leq i \leq a)$. O hipergrafo $\mathcal{X}^{t+1}=\left(X^{t+1}, \mathcal{M}^{t+1}\right)$ será o amálgama entre $\mathcal{A}_{\left|X_{t}^{t}\right|, k, g-1}=\left(Y^{t}, \mathcal{N}^{t}\right)$ dado pela hipótese de indução e $\mathcal{X}^{t}$ (Figura 5.2). Assim, $\mathcal{X}^{t+1}=\mathcal{A}_{\left|X_{t}^{t}\right|, k, g-1} * \mathcal{X}^{t}$ é um $p$-grafo $a$-partido; chame suas partes de $X_{i}^{t+1}(i \in[a])$. Além disso, $\mathcal{X}^{t+1}$ é constituído de $\left|\mathcal{N}^{t}\right|$ cópias de $\mathcal{X}^{t}$ costuradas através das partes $X_{t}^{t}$, que passam a formar a parte $X_{t}^{t+1}$. Faça $\mathcal{A}_{p, k, g}=\mathcal{X}^{a+1}$.

Agora é provado que $\mathcal{A}_{p, k, g} \in \mathcal{G}_{p, k, g}$. Já foi notado que qualquer hipergrafo $\mathcal{X}^{t}(t \in[a+1])$ é um $p$-grafo $a$-partido, pois a sequência começa com um $p$-grafo $a$-partido $\mathcal{X}^{1}$ e a amalgamação mantém essas características, como visto na Definição 5.1.2.

Também é verdade que todo hipergrafo da sequência tem cintura maior que $g$. Para ver isso, note que $\mathcal{X}^{1}$ não contém circuitos. Seja $t \geq 1$ e suponha indutivamente que $\operatorname{cin}\left(\mathcal{X}^{t}\right)>g$. Temse que $\operatorname{cin}\left(\mathcal{A}_{\left|X_{t}^{t}\right|, k, g-1}\right)>g-1$ e que $\mathcal{X}^{t+1}=\mathcal{A}_{\left|X_{t}^{t}\right|, k, g-1} * \mathcal{X}^{t}$. Será provado que $\operatorname{cin}\left(\mathcal{X}^{t+1}\right)>g$. Suponha por absurdo que $\mathcal{X}^{t+1}$ tem um circuito de comprimento $q \leq g$. As arestas de $\mathcal{X}^{t+1}$ são da forma $(M, N)$ com $M \in \mathcal{M}^{t}$ e $N \in \mathcal{N}^{t}$. Seja $\left(\left(M_{1}, N_{1}\right),\left(M_{2}, N_{2}\right), \ldots,\left(M_{q}, N_{q}\right)\right)$ um circuito de comprimento $q$ em $\mathcal{X}^{t+1}$.

Se $N_{1}=N_{2}=\cdots=N_{q}$, então o circuito está contido numa cópia de $\mathcal{X}^{t}$ pela Definição 5.1.2. Mas a hipótese é de que $\mathcal{X}^{t}$ não pode ter circuito de comprimento menor que ou igual a $g$, uma contradição.

Se nem todas arestas do circuito estão numa mesma cópia de $\mathcal{X}^{t}$, então toda vez que se muda de cópia, as arestas devem se intersectar num vértice da parte $X_{t}^{t+1}$, que foi por onde as cópias foram amalgamadas. A parte $X_{t}^{t+1}$ é composta pelos vértices de $\mathcal{A}_{\left|X_{t}^{t}\right|, k, g-1}$ e duas cópias de $\mathcal{X}^{t}$ que se intersectam correspondem a arestas de $\mathcal{A}_{\left|X_{t}^{t}\right|, k, g-1}$ que se intersectam (Figura 5.3). O que será feito é, a partir das intersecções entre duas arestas do circuito que mudam de cópia de $\mathcal{X}^{t}$, encontrar um 
circuito pequeno em $\mathcal{A}_{\left|X_{t}^{t}\right|, k, g-1}$.

$$
\mathcal{A}_{\left|X_{t}^{t}\right|, k, g-1} * \mathcal{X}^{t}
$$

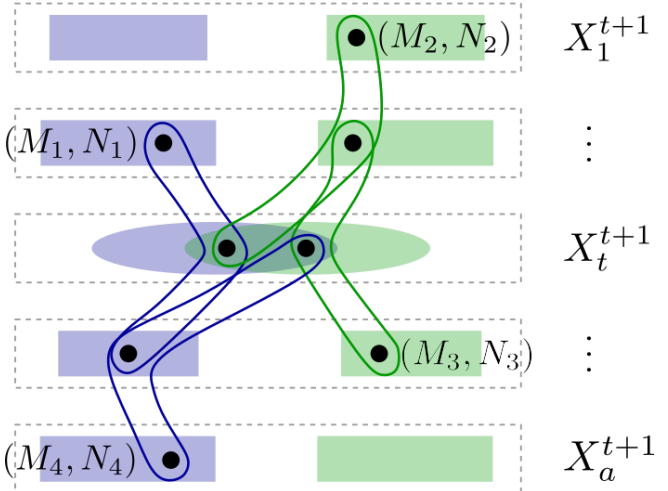

Figura 5.3: Um circuito em $\mathcal{X}^{t+1}$ induz um circuito em $\mathcal{A}_{\mid X_{t}^{t \mid}, k, g-1}$.

Suponha, sem perda de generalidade, que $N_{1} \neq N_{2}$. Como as arestas $\left(M_{1}, N_{1}\right)$ e $\left(M_{2}, N_{2}\right)$ se intersectam, mas estão em cópias de $\mathcal{X}^{t}$ que são disjuntas a menos da parte $X_{t}^{t}$, a intersecção deve ser o vértice $f_{N_{1}}\left(X_{t}^{t} \cap M_{1}\right)=f_{N_{2}}\left(X_{t}^{t} \cap M_{2}\right)$ que está na parte $X_{t}^{t+1}$. A última aresta $\left(M_{q}, N_{q}\right)$ também deve intersectar $\left(M_{1}, N_{1}\right)$ e deve ser num vértice diferente de $f_{N_{1}}\left(X_{t}^{t} \cap M_{1}\right)$, portanto tem-se que $N_{q}=N_{1}$.

Seja $\boldsymbol{N}=\left(N_{1}, \ldots, N_{q}\right)$ e tome $\left(N_{i_{1}}, \ldots, N_{i_{\ell}}\right)$ a subsequência de $\boldsymbol{N}$ com os menores índices possíveis tal que todos seus termos são distintos, que $N_{i_{j+1}}$ é o próximo termo na sequência $N$ que é distinto de $N_{i_{j}}(j \in[\ell-1])$ e que, sendo $N_{i_{\ell+1}}$ o próximo termo em $\boldsymbol{N}$ diferente de $N_{i_{\ell}}$, temse $N_{i_{\ell+1}}=N_{i_{1}}$ (Figura 5.4). Como $N_{q}=N_{1}$, tem-se que $N_{q}$ certamente não pertence à subsequência, portanto $\ell \leq q-1$.

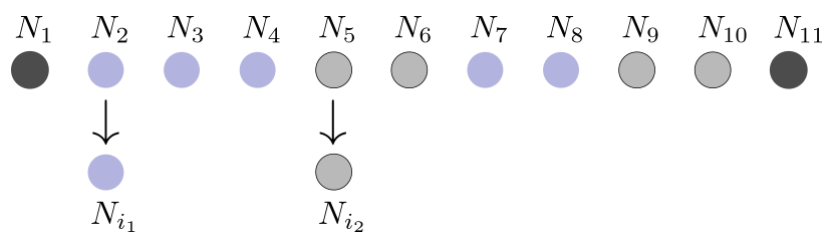

Figura 5.4: Círculos iguais correspondem a arestas iguais de $\mathcal{A}_{\mid X_{t \mid, k, g-1}^{t}}$. Em cima se tem $\boldsymbol{N}$, embaixo a subsequência.

Agora prova-se que $\left(N_{i_{1}}, \ldots, N_{i_{\ell}}\right)$ é um circuito em $\mathcal{A}_{\left|X_{t}^{t}\right|, k, g-1}$. Pela forma que a subsequência foi definida, existe no circuito um segmento da forma

$$
\left(\left(M_{i_{1 b}}, N_{i_{1}}\right),\left(M_{i_{2 a}}, N_{i_{2}}\right), \ldots,\left(M_{i_{2 b}}, N_{i_{2}}\right),\left(M_{i_{3 a}}, N_{i_{3}}\right), \ldots,\left(M_{i_{\ell b}}, N_{i_{\ell}}\right),\left(M_{i_{(\ell+1) a}}, N_{i_{\ell+1}}\right)\right) .
$$

Uma aresta $\left(M_{i_{j a}}, N_{i_{j}}\right)$ é a primeira de um segmento do circuito contido na cópia de $\mathcal{X}^{t}$ correspondente à aresta $N_{i_{j}}$ e $\left(M_{i_{j b}}, N_{i_{j}}\right)$ é a última desse segmento.

Tem-se, para $j \in[\ell]$, que $N_{i_{j}}$ e $N_{i_{j+1}}$ se intersectam em $f_{N_{i_{j}}}\left(X_{t}^{t} \cap M_{i_{j b}}\right)=f_{N_{i_{j+1}}}\left(X_{t}^{t} \cap M_{i_{(j+1) a}}\right)$. O argumento é o mesmo utilizado anteriormente, para concluir que $N_{1}=N_{q}$. Além disso, para cada $j$, essa interseç̧ão entre $N_{i_{j}}$ e $N_{i_{j+1}}$ é em um vértice distinto, pois sendo (5.1) um segmento de um circuito, é possível escolher um representante distinto para cada intersecção entre pares de arestas consecutivas e $f_{N_{i_{j}}}\left(X_{t}^{t} \cap M_{i_{j b}}\right)=f_{N_{i_{j+1}}}\left(X_{t}^{t} \cap M_{i_{(j+1) a}}\right)$ é a única intersecção entre $\left(M_{i_{j b}}, N_{i_{j}}\right)$ 
e $\left(M_{i_{(j+1) a}}, N_{i_{j+1}}\right)$. Assim, $\left(Y^{t}, \mathcal{N}^{t}\right)$ tem um circuito de comprimento menor ou igual a $g-1$, uma contradição.

Falta checar que $\chi\left(\mathcal{A}_{p, k, g}\right) \geq k$. Tome uma $(k-1)$-coloração dos vértices de $\mathcal{A}_{p, k, g}$, quer-se encontrar uma aresta monocromática. Sejam $\mathcal{A}_{p, k, g}=\left(X^{a+1}, \mathcal{M}^{a+1}\right)$ e $X_{t}^{a+1}(t \in[a])$ cada uma das partes de $X^{a+1}$. A ideia é que cada $X_{t}^{a+1}$ já foi a parte sobre a qual se fez a amalgamação. Assim, $X_{t}^{t+1}$ foi, após a $t$-ésima amalgamação, identificado com os vértices de $\mathcal{A}_{\left|X_{t}^{t}\right|, k, g-1}$. E, nas amalgamações seguintes, foram tomadas cópias disjuntas desses vértices. Portanto, $X_{t}^{a+1}$ é constituído de cópias disjuntas dos vértices de $\mathcal{A}_{\left|X_{t}^{t}\right|, k, g-1}$.

Assim, a coloração de $\mathcal{A}_{p, k, g}$ induzirá uma coloração com $k-1$ cores em cada $\mathcal{A}_{\left|X_{t}^{t}\right|, k, g-1}$, $\operatorname{com}(t \in[a])$. Essas colorações definem arestas monocromáticas nos $\mathcal{A}_{\left|X_{t}^{t}\right|, k, g-1}$. Essas arestas induzirão, em $\mathcal{A}_{p, k, g}$, um subgrafo que é $a$-partido, com partes monocromáticas e que contem uma cópia de $\mathcal{X}^{1}$ (Figura 5.5). Usando a propriedade das arestas de $\mathcal{X}^{1}$, será obtida uma aresta monocromática em $\mathcal{A}_{p, k, g}$.
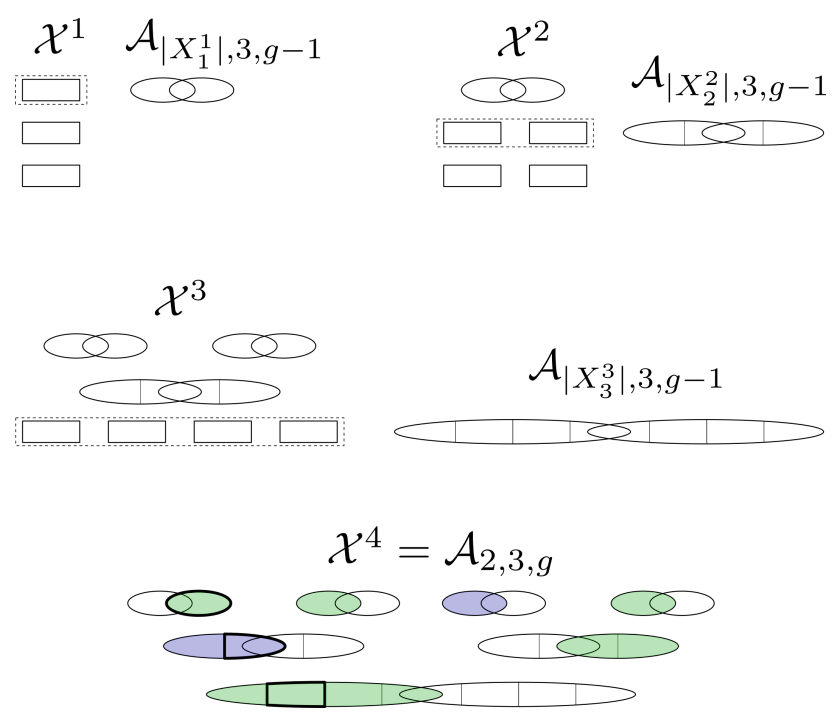

Figura 5.5: Esquema da construção de $\mathcal{A}_{2,3, g}$. Uma 2-coloração de $\mathcal{A}_{2,3, g}$ gera arestas monocromáticas em cada $\mathcal{A}_{\left|X_{t}^{t}\right|, 3, g-1}$. Está destacada a cópia de $\mathcal{X}^{1}$ em que cada parte é monocromática.

De forma mais precisa, serão indicados os vértices de $\mathcal{A}_{p, k, g}$ que formam o subgrafo $a$-partido em que cada parte é monocromática. Depois serão indicados os vértices da cópia de $\mathcal{X}^{1}$ contida nesse subgrafo. É importante lembrar que os vértices do amálgama são definidos como pares ordenados ou como a imagem de uma certa bijeção (Definição 5.1.2) e que os vértices de $\mathcal{A}_{\left|X_{t}^{t}\right|, k, g-1}$ foram chamados de $Y^{t}$ e as arestas de $\mathcal{N}^{t}$.

Primeiramente, olhe para a parte $X_{a}^{a+1}$. Como $\mathcal{A}_{p, k, g}=\mathcal{A}_{\left|X_{a}^{a}\right|, k, g-1} * \mathcal{X}^{a}$, os vértices em $X_{a}^{a+1}$ são $Y^{a}$. Assim, a coloração de $\mathcal{A}_{p, k, g}$ induz uma coloração em $\mathcal{A}_{\left|X_{a}^{a}\right|, k, g-1}$. Como $\chi\left(\mathcal{A}_{\left|X_{a}^{a}\right|, k, g-1}\right) \geq k$, existe uma aresta monocromática, que será chamada de $N_{a}$. Os vértices de $N_{a}$ estão contidos em $X_{a}^{a+1}$. Agora considere a parte $X_{a-1}^{a+1}$. Dentro dela, há diversas cópias de $Y^{a-1}$, uma para cada aresta de $\mathcal{N}^{a}$. Considere a cópia que corresponde à aresta $N_{a}$, ela é constituída pelos vértices $Y^{a-1} \times\left\{N_{a}\right\}$. Esta cópia induz uma $(k-1)$-coloração de $\mathcal{A}_{\left|X_{a-1}^{a-1}\right|, k, g-1}$. Assim, também há uma aresta de $\mathcal{N}^{a-1}$ que é monocromática, ela será $N_{a-1}$. Note que $N_{a-1}$ não está contida em $X_{a-1}^{a+1}$, mas sim em $Y^{a-1}$. Suponha que foi determinada a sequência de arestas $\left(N_{a}, N_{a-1} \ldots, N_{t+1}\right)$, com $t \geq 1$ e $N_{i} \in \mathcal{N}^{i}(t+1 \leq i \leq a)$. Para determinar a aresta $N_{t} \in \mathcal{N}^{t}$, considera-se a parte $X_{t}^{a+1}$ e, dentro dela, a cópia de $Y^{t}$ dada por $Y^{t} \times\left\{N_{t+1}\right\} \times \cdots \times\left\{N_{a}\right\}$. Esta cópia induz uma coloração de $\mathcal{A}_{\left|X_{t}^{t}\right|, k, g-1}$. 
Sob essa coloração, existe uma aresta monocromática, que será $N_{t}$.

Foi escolhida uma sequência $\left(N_{1}, N_{2}, \ldots, N_{a}\right)$, com $N_{t} \in \mathcal{N}^{t}$. Com essa sequência, podem ser definidos os conjuntos $Z_{t} \subset X_{t}^{a+1}(t \in[a])$, que serão as partes do subgrafo de $\mathcal{A}_{p, k, g}$ em que cada parte é monocromática. Para $t=a$ se tinha que $N_{a} \subset X_{a}^{a+1}$, então

$$
Z_{a}=N_{a}
$$

Para os demais valores de $t$, define-se

$$
Z_{t}=\left\{\left(v, N_{t+1}, \ldots, N_{a}\right): v \in N_{t}\right\} \subset X_{t}^{a+1}
$$

Como $Z_{t}$ é a cópia de $N_{t}$ dentro da cópia de $Y^{t}$ que induziu a coloração de $\mathcal{A}_{\left|X_{t}^{t}\right|, k, g-1}$, tem-se que $Z_{t}$ é monocromático (Figura 5.6).

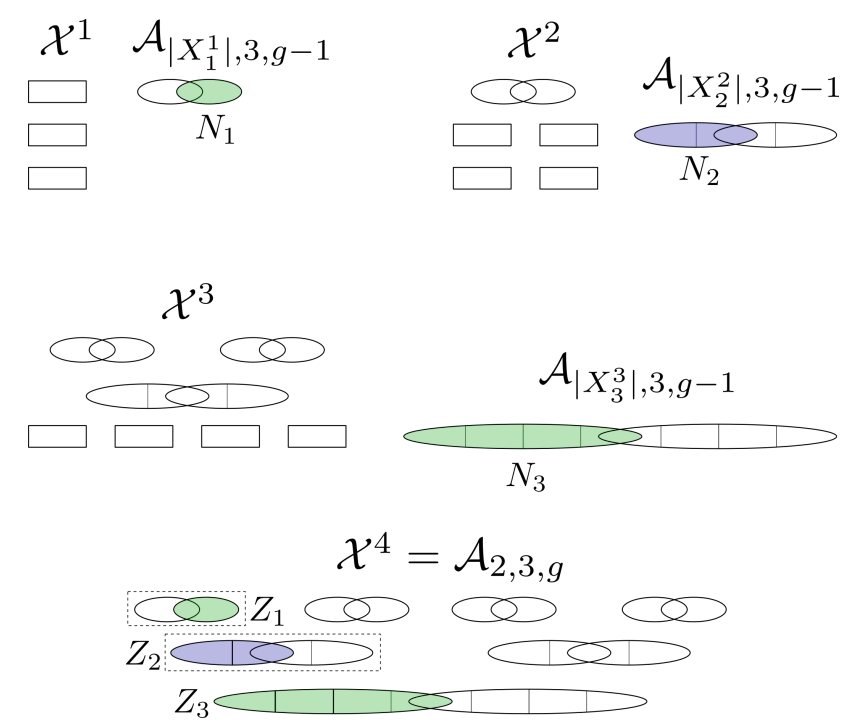

Figura 5.6: Estão marcadas as arestas monocromáticas $N_{t}(t \in[3])$, segundo a coloração induzida pelas cópias circuladas de cada $\mathcal{A}_{\mid X_{t \mid, 3, g-1}^{t}}$. Em $\mathcal{A}_{2,3, g}$, estão marcados os conjuntos monocromáticos $Z_{t}$.

Agora serão definidos os vértices da cópia de $\mathcal{X}^{1}$ dentro do subgrafo gerado pelos $Z_{t}$, para todo $t \in[a]$. Para a construção de $\mathcal{A}_{p, k, g}$, começa-se com $\mathcal{X}^{1}$ e são feitas $a$ amalgamações. Seguindo as amalgamações, na primeira, a aresta $N_{1} \in \mathcal{N}^{1}$ indica uma cópia de $\mathcal{X}^{1} \mathrm{em} \mathcal{X}^{2}$. Essa cópia é formada pelos vértices $X_{t}^{1} \times\left\{N_{1}\right\}$ para $t \geq 2$ e $f_{N_{1}}\left(X_{1}^{1}\right)=N_{1}$, onde $f_{N_{1}}$ é a bijeção entre $X_{1}^{1}$ e $N_{1} \in \mathcal{N}^{1}$. Depois, $N_{2}$ indica uma cópia de $\mathcal{X}^{2}$ em $\mathcal{X}^{3}$ e, dentro desta cópia, tem-se a cópia de $\mathcal{X}^{1}$ indicada por $N_{1}$. Assim, em $\mathcal{X}^{3}$, a cópia de $\mathcal{X}^{1}$ tem vértices $f_{N_{1}}\left(X_{1}^{1}\right) \times\left\{N_{2}\right\}$, mais $f_{N_{2}}\left(X_{2}^{1} \times\left\{N_{1}\right\}\right) \subset N_{2}$ e $X_{t}^{1} \times\left\{N_{1}\right\} \times\left\{N_{2}\right\}$, para $t \geq 3$. Assim por diante, em $\mathcal{X}^{a+1}$, tem-se uma cópia de $\mathcal{X}^{1}$ (Figura 5.7) formada pelos vértices em

$$
f_{N_{1}}\left(X_{1}^{1}\right) \times\left\{N_{2}\right\} \times \cdots \times\left\{N_{a}\right\}=Z_{1},
$$

para as partes $2 \leq t \leq a-1$, tem-se

$$
f_{N_{t}}\left(X_{t}^{1} \times\left\{N_{1}\right\} \times \cdots \times\left\{N_{t-1}\right\}\right) \times\left\{N_{t+1}\right\} \times \cdots \times\left\{N_{a}\right\} \subset Z_{t},
$$

por fim,

$$
f_{N_{a}}\left(X_{a}^{1} \times\left\{N_{1}\right\} \times \cdots \times\left\{N_{a-1}\right\}\right) \subset Z_{a} .
$$



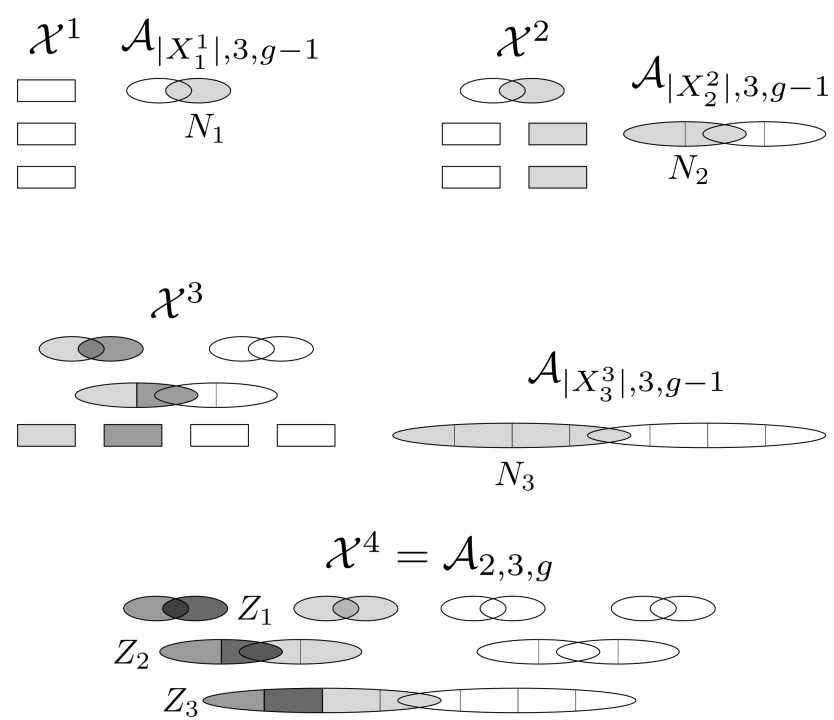

Figura 5.7: Em cada $\mathcal{X}^{t+1}(t \in[3])$, estão marcadas as cópia dos $\mathcal{X}^{t^{\prime}}\left(t^{\prime} \in[t]\right)$ anteriores indicadas pelas escolhas das arestas $N_{1}, \ldots, N_{t^{\prime}}$.

Como $a=(k-1)(p-1)+1$, existe $\omega \subset[a]$, com $|\omega|=p$, tal que qualquer conjunto $Z_{t}$, para $t \in \omega$, tem seus vértices coloridos de uma mesma cor. O grafo $\mathcal{X}^{1}$ foi escolhido de forma que, para qualquer escolha de $p$ partes, exista uma aresta contida nelas. Assim, na cópia de $\mathcal{X}^{1}$ contida em $Z_{1}, \ldots, Z_{a}$, considere as partes contidas em $Z_{t}$, com $t \in \omega$. Existe uma aresta da cópia de $\mathcal{X}^{1}$ que tem exatamente um vértice em cada uma destas partes. Portanto, tem-se uma aresta monocromática em $\mathcal{A}_{p, k, g}$.

Note que, nessa construção, mesmo para se construir o grafo $\mathcal{A}_{2, k, g} \in \mathcal{G}_{k, g}$, é preciso envolver hipergrafos.

\subsection{O número de vértices de $\mathcal{A}_{p, k, g}$}

O objetivo, agora, é estimar o número de vértices de $\mathcal{A}_{p, k, g}$. Quando $g=1$, para todo $k \geq 2$ e $p \geq 2$, sabe-se que $\mathcal{A}_{p, k, 1}$ é o clique $p$-uniforme em $a=(p-1)(k-1)+1$ vértices. Será feita uma indução em $g$, mas em vez de se estimar diretamente o número de vértices, a indução será feita para se estimar o número de arestas de $\mathcal{A}_{p, k, g}$. Depois, será utilizado o fato de o número de arestas de um $p$-grafo ser no máximo o número de arestas do clique, para se ter uma cota inferior no número de vértices. E será utilizado o fato de que, se todo vértice tem grau pelo menos 1, então o número de arestas de um $p$-grafo é pelo menos o número de vértices sobre $p$, para se encontrar uma cota superior do número de vértices. Assim, defina $m(p, k, g)$, com $p \geq 2, k \geq 2$ e $g \geq 1$, como o número de arestas de $\mathcal{A}_{p, k, g}$.

Para a indução em $g$, é preciso relacionar $m(p, k, g)$ com valores de $m\left(p^{\prime}, k^{\prime}, g-1\right)$ para certos $p^{\prime}$ e $k^{\prime}$. Para isso, note que, no amálgama $\mathcal{Y} * \mathcal{X}$, é tomada uma cópia do hipergrafo $\mathcal{X}$ para cada aresta de $\mathcal{Y}$. Assim, tem-se que o número de arestas do amálgama é o número de arestas de $\mathcal{X}$ multiplicado pelo número de arestas de $\mathcal{Y}$. O mesmo não ocorre com o número de vértices, pois as cópias não são disjuntas.

Na construção de $\mathcal{A}_{p, k, g}$, começa-se com um certo hipergrafo $\mathcal{X}^{1}=\left(X^{1}, \mathcal{M}^{1}\right)$ e depois se tem uma sequência de amálgamas $\mathcal{X}^{2}, \ldots, \mathcal{X}^{a+1}$. O hipergrafo $\mathcal{X}^{t+1}(t \in[a])$ é o amalgama entre $\mathcal{X}^{t}$ 
e $\mathcal{A}_{\left|X_{t}^{t}\right|, k, g-1}$. Sabendo disso e usando a ideia de que, no amálgama, os números de arestas de se multiplicam, tem-se uma relação entre $m(p, k, g)$ e os valores $m\left(\left|X_{t}^{t}\right|, k, g-1\right)(t \in[a])$. Essa relação é

$$
m(p, k, g)=\left|\mathcal{M}^{1}\right| \cdot m\left(\left|X_{1}^{1}\right|, k, g-1\right) \cdots m\left(\left|X_{a}^{a}\right|, k, g-1\right) .
$$

Os valores de $\left|X_{t}^{t}\right|(t \in[a])$ também precisam ser estimados. Cada $\left|X_{t}^{t}\right|$ será relacionado com os valores de $\left|X_{i}^{i}\right|(i \in[t-1])$. Foi dito que, no amálgama $\mathcal{Y} * \mathcal{X}$, o número de vértices de $\mathcal{X}$ não é apenas multiplicado pelo número de $\operatorname{arestas}$ de $\mathcal{Y}$, pois as cópias de $\mathcal{X}$ não são totalmente disjuntas. Porém olhando para as cópias de cada parte de $\mathcal{X}$, exceto pela parte sobre a qual se fez a amalgamação, as demais são cópias disjuntas. Então o tamanho de cada parte do amálgama, exceto pela parte amalgamada, é igual ao número de vértices da parte de $\mathcal{X}$ correspondente, multiplicado pelo número de arestas de $\mathcal{Y}$.

Na construção de $\mathcal{A}_{p, k, g}$, começa-se com um certo hipergrafo $\mathcal{X}^{1}$ e $\mathcal{X}^{t}=\mathcal{A}_{\left|X_{t-1}^{t-1}\right|, k, g-1} * \mathcal{X}^{t-1}$, uma amalgamação sobre a parte $t-1$. Assim, para cada $t \in[a]$ e todo $t \leq j \leq a$, a parte $X_{j}^{t}$ é constituída de $m\left(\left|X_{t-1}^{t-1}\right|, k, g-1\right)$ cópias disjuntas da parte $X_{j}^{t-1}$. Portanto, até se chegar em $\mathcal{X}^{t}$ o tamanho da $t$-ésima parte foi sempre sendo multiplicado por um certo número de arestas. Tem-se a seguinte relação entre $\left|X_{t}^{t}\right|$ e os demais valores $\left|X_{i}^{i}\right|(i \in[t-1])$,

$$
\left|X_{t}^{t}\right|=\left|X_{t}^{1}\right| \cdot m\left(\left|X_{1}^{1}\right|, k, g-1\right) \ldots m\left(\left|X_{t-1}^{t-1}\right|, k, g-1\right)
$$

As relações (5.2) e (5.3) serão utilizadas para estimar $m(p, k, g)$. Primeiro, é visto como obter uma cota inferior para o número de arestas de $\mathcal{A}_{p, k, g}$.

Proposição 5.3.1. Sejam $p \geq 2, k \geq 2$ e $g \geq 2$ inteiros e tome $a=(k-1)(p-1)+1$. Vale que $m(p, k, g) \geq((k-1) / 2) \uparrow^{g} a$.

Demonstração. Como foi dito, a prova é por indução em $g$. Sejam $p \geq 2$ e $k \geq 2$ inteiros. Para todo $g \geq 2$, serão usadas as seguintes cotas obtidas a partir de (5.2) e de (5.3) respectivamente.

$$
m(p, k, g)=\left|\mathcal{M}^{1}\right| \cdot m\left(\left|X_{1}^{1}\right|, k, g-1\right) \cdots m\left(\left|X_{a}^{a}\right|, k, g-1\right) \geq m\left(\left|X_{a}^{a}\right|, k, g-1\right) .
$$

E, para todo $2 \leq t \leq a$, tem-se

$$
\left|X_{t}^{t}\right|=\left|X_{t}^{1}\right| \cdot m\left(\left|X_{1}^{1}\right|, k, g-1\right) \ldots m\left(\left|X_{t-1}^{t-1}\right|, k, g-1\right) \geq m\left(\left|X_{t-1}^{t-1}\right|, k, g-1\right) .
$$

Para o caso base $g=2$, tem-se por (5.4) e pela definição de $\mathcal{A}_{\left|X_{a}^{a}\right|, k, 1}$ que

$$
m(p, k, 2) \geq m\left(\left|X_{a}^{a}\right|, k, 1\right)=\left(\begin{array}{c}
\left(\left|X_{a}^{a}\right|-1\right)(k-1)+1 \\
\left|X_{a}^{a}\right|
\end{array}\right) \geq\left(\frac{k-1}{2}\right)^{\left|X_{a}^{a}\right|},
$$

onde foi usado que $\left(\left|X_{a}^{a}\right|-1\right) /\left|X_{a}^{a}\right| \geq 1 / 2$, pois $\left|X_{a}^{a}\right| \geq 2$.

É preciso lidar com $\left|X_{a}^{a}\right|$. Prova-se por indução em $t \geq 2$, que $\left|X_{t}^{t}\right| \geq((k-1) / 2) \uparrow^{2}(t-1)$. Por (5.5) e pela definição de $\mathcal{A}_{\left|X_{1}^{1}\right|, k, 1}$, tem-se que

$$
\left|X_{2}^{2}\right| \geq m\left(\left|X_{1}^{1}\right|, k, 1\right) \geq\left(\frac{k-1}{2}\right)^{\left|X_{1}^{1}\right|} \geq \frac{k-1}{2}=\frac{k-1}{2} \uparrow^{2} 1 .
$$


Seja $t \geq 2$ e suponha que $\left|X_{t}^{t}\right| \geq((k-1) / 2) \uparrow^{2}(t-1)$. Analogamente, tem-se

$$
\left|X_{t+1}^{t+1}\right| \geq m\left(\left|X_{t}^{t}\right|, k, 1\right) \geq\left(\frac{k-1}{2}\right)^{\left|X_{t}^{t}\right|} \geq \frac{k-1}{2} \uparrow \frac{k-1}{2} \uparrow^{2}(t-1)=\frac{k-1}{2} \uparrow^{2} t .
$$

Portanto, por (5.6) e (5.7), tem-se que

$$
m(p, k, 2) \geq \frac{k-1}{2} \uparrow \frac{k-1}{2} \uparrow^{2}(a-1)=\frac{k-1}{2} \uparrow^{2} a .
$$

Seja $g \geq 3$ e suponha que, para todo $p \geq 2$ e $k \geq 2$, vale que $m(p, k, g-1) \geq((k-1) / 2) \uparrow^{g-1} a$, onde $a=(p-1)(k-1)+1$. Assim, usando (5.4),

$$
m(p, k, g) \geq m\left(\left|X_{a}^{a}\right|, k, g-1\right) \geq \frac{k-1}{2} \uparrow^{g-1}\left(\left(\left|X_{a}^{a}\right|-1\right)(k-1)+1\right) \geq \frac{k-1}{2} \uparrow^{g-1}\left|X_{a}^{a}\right| .
$$

Esse $\left|X_{a}^{a}\right|$ é diferente do $\left|X_{a}^{a}\right|$ do caso $g=2$, então é preciso estimá-lo novamente de forma semelhante. Será obtido, para todo $t \geq 2$, que $\left|X_{t}^{t}\right| \geq((k-1) / 2) \uparrow^{g}(t-1)$. Tem-se por (5.5) e (5.8), que

$$
\left|X_{2}^{2}\right| \geq m\left(\left|X_{1}^{1}\right|, k, g-1\right) \geq \frac{k-1}{2} \uparrow^{g-1}\left|X_{1}^{1}\right| \geq \frac{k-1}{2}=\frac{k-1}{2} \uparrow^{g} 1 .
$$

Seja $t \geq 2$ e suponha que $\left|X_{t}^{t}\right| \geq((k-1) / 2) \uparrow^{g}(t-1)$. Assim, usando (5.5) e (5.8), tem-se

$$
\left|X_{t+1}^{t+1}\right| \geq m\left(\left|X_{t}^{t}\right|, k, g-1\right) \geq \frac{k-1}{2} \uparrow^{g-1}\left|X_{t}^{t}\right| \geq \frac{k-1}{2} \uparrow^{g-1} \frac{k-1}{2} \uparrow^{g}(t-1)=\frac{k-1}{2} \uparrow^{g} t .
$$

Finalmente, de (5.8) e (5.9), tem-se

$$
m(p, k, g) \geq \frac{k-1}{2} \uparrow^{g-1} \frac{k-1}{2} \uparrow^{g}(a-1) \geq \frac{k-1}{2} \uparrow^{g} a .
$$

Para a cota inferior, os valores de $\left|\mathcal{M}^{1}\right|$ e $\left|X_{t}^{1}\right|$ foram sempre substituídos por 1. Porém não será possível fazer isso para a cota superior. Assim, observe o hipergrafo inicial $\mathcal{X}^{1}$ da construção de $\mathcal{A}_{p, k, g}$. Esse $p$-grafo $a$-partido é constituído de arestas disjuntas, uma para cada escolha de $p$ partes. Portanto, o número de arestas é $\left|\mathcal{M}^{1}\right|=\left(\begin{array}{l}a \\ p\end{array}\right)$. Para saber o tamanho de uma parte $\left|X_{t}^{1}\right|$, com $(t \in[a])$, é preciso contar quantas arestas passam por ela, haverá um vértice para cada aresta. Se uma dada escolha de $p$ partes deve conter a parte $X_{t}^{1}$, então faltam escolher $p-1$ partes dentre as outras $a-1$ partes. Portanto, tem-se $\left|X_{t}^{1}\right|=\left(\begin{array}{c}a-1 \\ p-1\end{array}\right)$.

Também será preciso provar que $m(p, k, g)$ é crescente em $p$ e em $k$.

Lema 5.3.2. Sejam $p \geq 2, k \geq 2$ e $g \geq 1$ inteiros. Vale que $m(p, k, g) \leq m(p+1, k, g)$.

Demonstração. Será feita uma indução em $g$. Sejam $p \geq 2$ e $k \geq 2$ inteiros quaisquer. Para $g=1$, tem-se da definição de $\mathcal{A}_{p, k, 1}$ que

$$
m(p, k, 1)=\left(\begin{array}{c}
(p-1)(k-1)+1 \\
p
\end{array}\right) .
$$


E da definição de $\mathcal{A}_{p+1, k, 1}$, tem-se que

$$
m(p+1, k, 1)=\left(\begin{array}{c}
p(k-1)+1 \\
p+1
\end{array}\right)=\left(\begin{array}{c}
(p-1)(k-1)+k \\
p+1
\end{array}\right) \geq\left(\begin{array}{c}
(p-1)(k-1)+2 \\
p+1
\end{array}\right) .
$$

Pela propriedade do binomial segundo a qual $\left(\begin{array}{c}a \\ b\end{array}\right)+\left(\begin{array}{c}a \\ b+1\end{array}\right)=\left(\begin{array}{c}a+1 \\ b+1\end{array}\right)$, desde que $(p-1)(k-1)+1 \geq p+1$, tem-se que $m(p+1, k, 1) \geq m(p, k, 1)$. Se $(p-1)(k-1)+1=p$, então $(k-2)(p-1)=0$. Como $p \geq 2$, tem-se $k=2$. Dessa forma, vale que $m(p+1, k, 1)=m(p, k, 1)=1$.

Seja $g \geq 2$ e suponha que $m(p+1, k, g-1) \geq m(p, k, g-1)$ para quaisquer $p \geq 2$ e $k \geq 2$. Para comparar $m(p+1, k, g)$ e $m(p, k, g)$ será utilizado (5.2). Note que os hipergrafos iniciais para $p$ e $p+1$, respectivamente $\mathcal{X}^{1}(p)$ e $\mathcal{X}^{1}(p+1)$, são distintos. Seja a o número de partes de $\mathcal{X}^{1}(p)$ e $a^{\prime}$ o número de partes de $\mathcal{X}^{1}(p+1)$. Tem-se $a=(p-1)(k-1)+1 \leq p(k-1)+1=a^{\prime}$. Utilizando-se de (5.2), pode-se escrever

$$
m(p, k, g)=\left|\mathcal{M}^{1}(p)\right| \cdot m\left(\left|X_{1}^{1}(p)\right|, k, g-1\right) \cdots m\left(\left|X_{a}^{a}(p)\right|, k, g-1\right) .
$$

E, para $p+1$, tem-se

$$
m(p+1, k, g) \geq\left|\mathcal{M}^{1}(p+1)\right| \cdot m\left(\left|X_{1}^{1}(p+1)\right|, k, g-1\right) \cdots m\left(\left|X_{a}^{a}(p+1)\right|, k, g-1\right) \cdot 1 \cdots 1 .
$$

Será provado que $\left|X_{t}^{t}(p+1)\right| \geq\left|X_{t}^{t}(p)\right|(t \in[a])$. É feita uma indução em $t$. Pela construção dos hipergrafos iniciais, tem-se que $\left|X_{1}^{1}(p+1)\right|=\left(\begin{array}{c}p(k-1) \\ p\end{array}\right) \geq\left(\begin{array}{c}(p-1)(k-1) \\ p-1\end{array}\right)=\left|X_{1}^{1}(p)\right|$. Seja $t \geq 2$ e suponha que para todo $t^{\prime} \leq t-1$, vale que $\left|X_{t^{\prime}}^{t^{\prime}}(p+1)\right| \geq\left|X_{t^{\prime}}^{t^{\prime}}(p)\right|$. Utilizado (5.3) e que $\left|X_{1}^{1}\right|=\left|X_{t}^{1}\right|$, isto é, que no grafo inicial todas partes tem o mesmo tamanho, tem-se

$$
\left|X_{t}^{t}(p+1)\right|=\left|X_{1}^{1}(p+1)\right| \cdot m\left(\left|X_{1}^{1}(p+1)\right|, k, g-1\right) \cdots m\left(\left|X_{t-1}^{t-1}(p+1)\right|, k, g-1\right) .
$$

Utilizando as duas hipóteses de indução, em $t$ e em $g$, tem-se

$$
\left|X_{t}^{t}(p+1)\right| \geq\left|X_{1}^{1}(p)\right| \cdot m\left(\left|X_{1}^{1}(p)\right|, k, g-1\right) \cdots m\left(\left|X_{t-1}^{t-1}(p)\right|, k, g-1\right)=\left|X_{t}^{t}(p)\right| .
$$

Agora, continua-se o passo da indução em $g$. Em (5.11), utilize que $\left|\mathcal{M}^{1}(p+1)\right| \geq\left|\mathcal{M}^{1}(p)\right|$, que segue da definição dos hipergrafos iniciais. E utilize que $\left|X_{t}^{t}(p+1)\right| \geq\left|X_{t}^{t}(p)\right|(t \in[a])$ e a hipótese de indução, para notar que $m\left(\left|X_{t}^{t}(p+1)\right|, k, g-1\right) \geq m\left(\left|X_{t}^{t}(p)\right|, k, g-1\right)$. Conclui-se que $m(p+1, k, g) \geq m(p, k, g)$.

De forma semelhante, prova-se que $m(p, k, g)$ é crescente em $g$.

Lema 5.3.3. Sejam $p \geq 2, k \geq 2$ e $g \geq 1$ inteiros. Vale que $m(p, k, g) \leq m(p, k, g+1)$.

Demonstração. É feita uma indução em $g$. Sejam $p \geq 2$ e $k \geq 2$ inteiros quaisquer. Para $g=1$, note que $\left|\mathcal{M}^{1}\right|=\left(\begin{array}{l}a \\ p\end{array}\right)=m(p, k, 1)$ por definição do hipergrafo inicial e de $\mathcal{A}_{p, k, 1}$. Assim, por (5.2),

$$
m(p, k, 2)=\left|\mathcal{M}^{1}\right| \cdot m\left(\left|X_{1}^{1}\right|, k, 1\right) \cdots m\left(\left|X_{a}^{a}\right|, k, 1\right) \geq\left|\mathcal{M}^{1}\right|=m(p, k, 1) .
$$

Seja $g \geq 2$ e suponha que $m(p, k, g) \geq m(p, k, g-1)$ para quaisquer $p \geq 2$ e $k \geq 2$. Agora serão comparados $m(p, k, g+1)$ e $m(p, k, g)$. Observe que, como $p$ e $k$ são os mesmos, tem-se o mesmo hipergrafo inicial, $\mathcal{X}^{1}(g)=\mathcal{X}^{1}(g+1)$. Porém, para $2 \leq t \leq a$, os hipergrafos $\mathcal{X}^{t}(g)$ e $\mathcal{X}^{t}(g+1)$ são 
distintos, uma vez que cada $\mathcal{X}^{t-1}(g)$ é amalgamado com $\mathcal{A}_{\left|X_{t-1}^{t-1}(g)\right|, k, g-1}$, ao passo que $\mathcal{X}^{t-1}(g+1)$ é amalgamado com $\mathcal{A}_{\left|X_{t-1}^{t-1}(g+1)\right|, k, g}$. Feita essa observação, utilize (5.2) para escrever

$$
m(p, k, g+1)=\left|\mathcal{M}^{1}\right| \cdot m\left(\left|X_{1}^{1}(g+1)\right|, k, g\right) \cdots m\left(\left|X_{a}^{a}(g+1)\right|, k, g\right) .
$$

E, para $m(p, k, g)$, tem-se

$$
m(p, k, g)=\left|\mathcal{M}^{1}\right| \cdot m\left(\left|X_{1}^{1}(g)\right|, k, g-1\right) \cdots m\left(\left|X_{a}^{a}(g)\right|, k, g-1\right) .
$$

Agora, é preciso comparar $\left|X_{t}^{t}(g)\right| \operatorname{com}\left|X_{t}^{t}(g+1)\right|$, para $t \in[a]$. Já foi visto que $\mathcal{X}^{1}(g)=\mathcal{X}^{1}(g+1)$, $\operatorname{assim}\left|X_{1}^{1}(g)\right|=\left|X_{1}^{1}(g+1)\right|$. Seja $t \geq 2$ e suponha que, para $t^{\prime} \leq t-1$, tem-se $\left|X_{t^{\prime}}^{t^{\prime}}(g+1)\right| \geq\left|X_{t^{\prime}}^{t^{\prime}}(g)\right|$. Utilize (5.3) para escrever

$$
\left|X_{t}^{t}(g+1)\right|=\left|X_{1}^{1}\right| \cdot m\left(\left|X_{1}^{1}(g+1)\right|, k, g\right) \cdots m\left(\left|X_{t-1}^{t-1}(g+1)\right|, k, g\right) .
$$

Pela hipótese da indução em $t$ e usando o Lema 5.3.2, vale que

$$
\left|X_{t}^{t}(g+1)\right| \geq\left|X_{1}^{1}\right| \cdot m\left(\left|X_{1}^{1}(g)\right|, k, g\right) \cdots m\left(\left|X_{t-1}^{t-1}(g)\right|, k, g\right) .
$$

Utilizando a hipótese da indução em $g$, tem-se

$$
\left|X_{t}^{t}(g+1)\right| \geq\left|X_{1}^{1}\right| \cdot m\left(\left|X_{1}^{1}(g)\right|, k, g-1\right) \cdots m\left(\left|X_{t-1}^{t-1}(g)\right|, k, g-1\right)=\left|X_{t}^{t}(g)\right| .
$$

Continua-se o passo da indução em $g$, de forma semelhante ao passo da indução em $t$. Sabendo que $\left|X_{t}^{t}(g+1)\right| \geq\left|X_{t}^{t}(g)\right|(t \in[a])$, que $m(p, k, g)$ é crescente em $p$ e utilizando a hipótese de indução em $g$, tem-se

$$
m\left(\left|X_{t}^{t}(g+1)\right|, k, g\right) \geq m\left(\left|X_{t}^{t}(g)\right|, k, g\right) \geq m\left(\left|X_{t}^{t}(g)\right|, k, g-1\right) .
$$

Finalmente, use esse resultado em (5.12) para obter que $m(p, k, g+1) \geq m(p, k, g)$.

A última observação antes de calcular uma cota superior para $m(p, k, g)$ é sobre algumas operações que podem ser feitas com as flechas de Knuth.

Lema 5.3.4. Sejam $x, y, c \geq 2$ inteiros. Valem as seguintes relações.

1. Para $b \geq 2$, tem-se $\left(x \uparrow^{b} y\right)^{c} \leq x \uparrow^{b}(y+c)$.

2. Para $b \geq 1$, tem-se $c\left(x \uparrow^{b} y\right) \leq x \uparrow^{b}(y+c)$.

3. Para $b \geq 1$, tem-se $\left(x \uparrow^{b} y\right)^{c} \leq x \uparrow^{b}(y c)$.

Demonstração. Primeiro, prova-se (1). Será feita uma indução em $c$. Quando $c=2$, utiliza-se que $z^{2} \leq 2^{z}(z \neq 3)$ e que $\left(x \uparrow^{b} y\right) \geq 4$ para se escrever que $\left(x \uparrow^{b} y\right)^{2} \leq 2^{\left(x \uparrow^{b} y\right)}$. Depois, note que

$$
\begin{aligned}
\left(x \uparrow^{b} y\right)^{2} & \leq 2^{\left(x \uparrow^{b} y\right)} \leq x \uparrow^{b-1}\left(x \uparrow^{b} y\right)=x \uparrow^{b}(y+1) \\
& \leq x \uparrow^{b}(y+2),
\end{aligned}
$$

onde foi usado que $x \geq 2$ e que $b-1 \geq 1$. 
Seja $c \geq 3$ e suponha que, para quaisquer $x, y, b \geq 2$, vale que $\left(x \uparrow^{b} y\right)^{c-1} \leq x \uparrow^{b}(y+c-1)$. Faça

$$
\begin{aligned}
\left(x \uparrow^{b} y\right)^{c} & =\left(x \uparrow^{b} y\right) \cdot\left(x \uparrow^{b} y\right)^{c-1} \leq\left(x \uparrow^{b} y\right) \cdot\left(x \uparrow^{b}(y+c-1)\right) \\
& \leq\left(x \uparrow^{b}(y+c-1)\right)^{2} \leq x \uparrow^{b}(y+c),
\end{aligned}
$$

onde a última desigualdade vale pelo que foi visto em (5.13).

Para provar (2), considere primeiro o caso $b=1$. Tem-se

$$
c(x \uparrow y)=c \cdot x^{y} \leq x^{c} \cdot x^{y}=x \uparrow(y+c),
$$

onde foi usado que $x \geq 2$.

Para $b \geq 2$, tem-se

$$
c\left(x \uparrow^{b} y\right) \leq\left(x \uparrow^{b} y\right)^{c} \leq x \uparrow^{b}(y+c),
$$

onde a primeira desigualdade vale pois $x \uparrow^{b} y>1$ e a segunda vale pela relação (1).

Por fim, prova-se (3). Separadamente, cheque o caso $b=1$. Tem-se

$$
(x \uparrow y)^{c}=\left(x^{y}\right)^{c}=x^{y c}=x \uparrow(y c) .
$$

Para $b \geq 2$, utilize a relação (1). Tem-se

$$
\left(x \uparrow^{b} y\right)^{c} \leq x \uparrow^{b}(y+c) \leq x \uparrow^{b}(y c)
$$

onde a última desigualdade vale, pois $c /(c-1) \leq 2 \leq y$, quando $c \geq 2$.

Prova-se agora uma cota superior para $m(p, k, g)$.

Proposição 5.3.5. Sejam $p \geq 2, k \geq 2$ e g $\geq 1$ inteiros. Vale que $m(p, k, g) \leq(3(k-1)) \uparrow^{g}(2 k p)$.

Demonstração. A prova é por indução em $g$. Para todo $p \geq 2$ e $k \geq 2$, o $p$-grafo $\mathcal{A}_{p, k, 1}$ tem $a$ vértices e é completo. Assim,

$$
m(p, k, 1)=\left(\begin{array}{c}
(p-1)(k-1)+1 \\
p
\end{array}\right) \leq\left(\begin{array}{c}
p(k-1) \\
p
\end{array}\right) \leq(e(k-1))^{p} \leq(3(k-1)) \uparrow(2 k p) .
$$

Suponha $g \geq 2$. Para fazer a indução em $g$, é preciso relacionar $m(p, k, g) \operatorname{com} m\left(p^{\prime}, k^{\prime}, g-1\right)$ para certos valores de $p^{\prime}$ e $k^{\prime}$. Assim, utiliza-se (5.2), que $\left|\mathcal{M}^{1}\right|=m(p, k, 1)$ e os Lemas 5.3.2 e 5.3.3 para se escrever que

$$
m(p, k, g)=\left|\mathcal{M}^{1}\right| \cdot m\left(\left|X_{1}^{1}\right|, k, g-1\right) \cdots m\left(\left|X_{a}^{a}\right|, k, g-1\right) \leq m\left(\left|X_{a}^{a}\right|, k, g-1\right)^{a+1} .
$$

E utiliza-se (5.3), que $\left|X_{t}^{1}\right| \leq m(p, k, 1)$ e os Lemas 5.3.2 e 5.3.3 para se obter que vale para todo $2 \leq t \leq a$, que

$$
\left|X_{t}^{t}\right| \leq m\left(\left|X_{t-1}^{t-1}\right|, k, g-1\right)^{t}
$$

Nota-se que será preciso fazer uma indução em $t$ para relacionar $\left|X_{t}^{t}\right|$ com o valor conhecido $\left|X_{1}^{1}\right|$. 
Seja $g \geq 2$ e suponha que $m(p, k, g-1) \geq(3(k-1)) \uparrow^{g-1} 2 k p$ para todo $p, k \geq 2$. Sejam $p, k \geq 2$ inteiros quaisquer e considere $m(p, k, g)$. Por (5.14) e usando a hipótese da indução, tem-se que

$$
m(p, k, g) \leq m\left(\left|X_{a}^{a}\right|, k, g-1\right)^{a+1} \leq\left((3(k-1)) \uparrow^{g-1}\left(2 k\left|X_{a}^{a}\right|\right)\right)^{a+1} .
$$

Como $g-1 \geq 1$, pode-se utilizar a relação 3 do Lema 5.3.4 para concluir que

$$
m(p, k, g) \leq(3(k-1)) \uparrow^{g-1}\left((a+1) 2 k\left|X_{a}^{a}\right|\right) .
$$

É preciso provar que $(a+1) 2 k\left|X_{a}^{a}\right| \leq(3(k-1)) \uparrow^{g}(2 k p-1)$. Será utilizada a relação (5.15) para lidar com $(a+1) 2 k\left|X_{a}^{a}\right|$. Mais especificamente, é provado, para todo $t \in[a]$, que

$$
(t+1) 2 k\left|X_{t}^{t}\right|+2 k \cdot \sum_{i=t+2}^{a+1} i \leq(3(k-1)) \uparrow^{g}(2 k p-a+t-1) .
$$

É feita uma indução em $t$. Para $t=1$, tem-se

$$
2 \cdot 2 k\left|X_{1}^{1}\right|+2 k \cdot \sum_{i=3}^{a+1} i \leq 4 k \cdot\left(\begin{array}{l}
a-1 \\
p-1
\end{array}\right)+2 k(a+1)(a-1) \leq 4 k \cdot(3(k-1))^{p-1}+2 k a^{2} .
$$

Quer-se mostrar que $4 k \cdot(3(k-1))^{p-1}+2 k a^{2} \leq(3(k-1)) \uparrow^{g}(2 k p-a)$, onde $p, k$ e $g$ estão fixos e valem pelo menos 2 . Note que

$$
(3(k-1)) \uparrow^{g}(2 k p-a) \geq(3(k-1)) \uparrow(2 k p-a) \geq(3(k-1))^{2 p+1} .
$$

Assim, basta provar que $4 k \cdot(3(k-1))^{p-1}+2 k a^{2} \leq(3(k-1)) \leq(3(k-1))^{2 p+1}$

Primeiro considere $4 k \cdot(3(k-1))^{p-1}$. Tem-se $4 k \leq(3(k-1))^{2}$, pois note que vale caso $k=2 \mathrm{e}$, para $k \geq 3$, vale que $(k-1)^{2} \geq 2(k-1) \geq k$. Portanto,

$$
4 k \cdot(3(k-1))^{p-1} \leq(3(k-1))^{p+1}=\frac{(3(k-1))^{2 p+1}}{(3(k-1))^{p}} \leq \frac{(3(k-1))^{2 p+1}}{2} .
$$

Agora considere $2 k a^{2}$. Tem-se, para $k \geq 3$, que $a^{2} \leq(p(k-1))^{2} \leq(k-1)^{2 p}$ e que $2 k \leq 3(k-1)$. Assim, para $k \geq 3$, vale que

$$
2 k a^{2} \leq 3(k-1)^{2 p+1} \leq \frac{(3(k-1))^{2 p+1}}{3^{2 p}} \leq \frac{(3(k-1))^{2 p+1}}{2} .
$$

Substitua $k=2$ e note que essa relação também é válida.

Portanto, por (5.18), (5.19), (5.20) e (5.21), tem-se

$$
2 \cdot 2 k\left|X_{1}^{1}\right|+2 k \cdot \sum_{i=3}^{a+1} i \leq 4 k \cdot(3(k-1))^{p-1}+2 k a^{2} \leq(3(k-1))^{2 p+1} \leq(3(k-1)) \uparrow^{g}(2 k p-a) .
$$

Seja $t \geq 1$ e suponha que $(t+1) 2 k\left|X_{t}^{t}\right|+2 k \cdot \sum_{i=t+2}^{a+1} i \leq(3(k-1)) \uparrow^{g}(2 k p-a+t-1)$. 
Utilize (5.15) para escrever que

$$
(t+2) 2 k\left|X_{t+1}^{t+1}\right|+2 k \cdot \sum_{i=t+3}^{a+1} i \leq(t+2) 2 k \cdot m\left(\left|X_{t}^{t}\right|, k, g-1\right)^{t+1}+2 k \cdot \sum_{i=t+3}^{a+1} i .
$$

Pela hipótese da indução em $g$, tem-se

$$
(t+2) 2 k\left|X_{t+1}^{t+1}\right|+2 k \cdot \sum_{i=t+3}^{a+1} i \leq(t+2) 2 k\left((3(k-1)) \uparrow^{g-1}\left(2 k\left|X_{t}^{t}\right|\right)\right)^{t+1}+2 k \cdot \sum_{i=t+3}^{a+1} i .
$$

Usando a relação 3 do Lema 5.3.4, tem-se

$$
(t+2) 2 k\left|X_{t+1}^{t+1}\right|+2 k \cdot \sum_{i=t+3}^{a+1} i \leq 2 k \cdot \sum_{i=t+2}^{a+1} i \cdot\left((3(k-1)) \uparrow^{g-1}\left(2 k\left|X_{t}^{t}\right|(t+1)\right)\right) .
$$

Usando a relação 2 do Lema 5.3.4, tem-se

$$
(t+2) 2 k\left|X_{t+1}^{t+1}\right|+2 k \cdot \sum_{i=t+3}^{a+1} i \leq(3(k-1)) \uparrow^{g-1}\left(2 k\left|X_{t}^{t}\right|(t+1)+2 k \cdot \sum_{i=t+2}^{a+1} i\right) .
$$

Finalmente, aplica-se a hipótese da indução em $t$ para se obter

$$
\begin{aligned}
(t+2) 2 k\left|X_{t+1}^{t+1}\right|+2 k \cdot \sum_{i=t+3}^{a+1} i & \leq(3(k-1)) \uparrow^{g-1}(3(k-1)) \uparrow^{g}(2 k p-a+t-1) \\
& \leq(3(k-1)) \uparrow^{g}(2 k p-a+t) .
\end{aligned}
$$

Continue a indução em $g$. A partir de (5.16) e utilizando a relação (5.17) que foi provada acima, tem-se

$$
\begin{aligned}
m(p, k, g) & \leq(3(k-1)) \uparrow^{g-1}\left((a+1) 2 k\left|X_{a}^{a}\right|\right) \\
& \leq(3(k-1)) \uparrow^{g-1}(3(k-1)) \uparrow^{g}(2 k p-1)=(3(k-1)) \uparrow^{g}(2 k p) .
\end{aligned}
$$

Como foi dito, foram calculadas cotas para o número de arestas. Quanto ao número de vértices, tem-se o seguinte resultado.

Teorema 5.3.6. Sejam $p, k, g \geq 2$ inteiros. Vale que

$$
\left(\frac{k-1}{2} \uparrow^{g}((p-1)(k-1)+1)\right)^{1 / p} \leq\left|\mathcal{A}_{p, k, g}\right| \leq p \cdot\left((3(k-1)) \uparrow^{g}(2 k p)\right) .
$$

Demonstração. Apenas note que nenhum vértice de $\mathcal{A}_{p, k, g}$ pode ser isolado. Quando $g=1$, temse $p$-grafos completos. Seja $g \geq 2$ e suponha que nenhum $\mathcal{A}_{p, k, g-1}$ tem vértices isolados. Na construção de $\mathcal{A}_{p, k, g}$, o grafo inicial $\mathcal{X}^{1}$ não possui vértices isolados pela forma que foi construído. Seja $t \in[a-1]$ e suponha que $\mathcal{X}^{t}$ não possui vértices isolados. Tem-se que $\mathcal{X}^{t+1}=\mathcal{A}_{\left|X_{t}^{t}\right|, k, g-1} * \mathcal{X}^{t}$. Então $\mathcal{X}^{t+1}$ é constituído de uma cópia de $\mathcal{X}^{t}$ para cada aresta de $\mathcal{A}_{\mid X_{t}^{t \mid, k, g-1}}$. Em cada cópia de $\mathcal{X}^{t}$, a parte $X_{t}^{t}$ foi substituída por uma aresta de $\mathcal{A}_{\mid X_{t}^{t \mid, k, g-1}}$. O hipergrafo $\mathcal{A}_{\left|X_{t}^{t \mid}\right| k, g-1}$ não tem vértices 
isolados, então todo vértice está em alguma cópia de $\mathcal{X}^{t}$. Além disso, $\mathcal{X}^{t}$ não tem vértices isolados. Assim, $\mathcal{X}^{t+1}$ também não tem vértices isolados.

Desta forma, tem-se

$$
m(p, k, g) \geq \frac{\left|\mathcal{A}_{p, k, g}\right|}{p} .
$$

Essa relação junto da Proposição 5.3.5 prova que $\left|\mathcal{A}_{p, k, g}\right| \leq p \cdot\left((3(k-1)) \uparrow^{g}(2 k p)\right)$.

Para a cota inferior, note que o número máximo de arestas em um $p$-grafo é o número de arestas do $p$-grafo completo. Tem-se

$$
m(p, k, g) \leq\left(\begin{array}{c}
\left|\mathcal{A}_{p, k, g}\right| \\
p
\end{array}\right) \leq\left|\mathcal{A}_{p, k, g}\right|^{p}
$$

Utilizando a Proposição 5.3.1, prova-se que $\left|\mathcal{A}_{p, k, g}\right| \geq\left(((k-1) /(2)) \uparrow^{g}((p-1)(k-1)+1)\right)^{1 / p}$.

Para o caso particular em que é buscado um grafo com cintura e número cromático grandes, tem-se o seguinte corolário.

Corolário 5.3.7. Sejam $p \geq 2, k \geq 5$ e $g \geq 2$ inteiros. Vale que

$$
(3(k-1)) \uparrow^{g}(4 k+2) \geq\left|\mathcal{A}_{2, k, g}\right| \geq\left(\frac{k-1}{2}\right) \uparrow^{g}(k-1) .
$$

Demonstração. Para a cota superior, basta utilizar o Teorema 5.3.6 e a relação 2 do Lema 5.3.4. Tem-se

$$
\left|\mathcal{A}_{2, k, g}\right| \leq 2\left((3(k-1)) \uparrow^{g}(4 k)\right) \leq(3(k-1)) \uparrow^{g}(4 k+2) .
$$

Para a cota inferior, utiliza-se o Teorema 5.3 .6 e que $\sqrt{x} \geq \log _{2}(x)$ quando $x \geq 16$. Tem-se

$$
\begin{aligned}
\left|\mathcal{A}_{2, k, g}\right| \geq\left(\frac{k-1}{2} \uparrow^{g} k\right)^{\frac{1}{2}} \geq \log _{2}\left(\frac{k-1}{2} \uparrow^{g} k\right) & \geq \log _{2}\left(2 \uparrow\left(\frac{k-1}{2} \uparrow^{g}(k-1)\right)\right) \\
& =\frac{k-1}{2} \uparrow^{g}(k-1) .
\end{aligned}
$$

A construção por amalgamação fornece grafos com cintura e número cromático grandes, que possuem um número Ackermanniano de vértices. O número de flechas cresce de acordo com $g$, a cintura desejada. Enquanto que, na construção de Kř̌iž, o número de flechas cresce de acordo com $\left\lceil\log _{2} g\right\rceil$. Dessa forma, o número de vértices do grafo obtido por amalgamação é maior que o obtido pela construção de Kříž.

A próxima construção que será apresentada, assim como a construção por amalgamação, pode ser utilizada para se obter hipergrafos uniformes com cintura e número cromático grandes. Além disso, assim como na construção de Kř̌̌ž, a contrução para o caso específico em que se busca um grafo não envolve hipergrafos. As estimativas do número de vértices que são apresentadas, no entanto, não permitem uma comparação com as construções anteriores. 


\title{
Capítulo 6
}

\section{As árvores aumentadas}

\author{
Construindo um hipergrafo com cintura e número cromático \\ grandes diretamente, a partir de um grafo.
}

Para construir $\mathcal{A}_{p, k, g}$, são feitas diversas amalgamações entre hipergrafos. Mesmo para $p=2$, é necessário envolver outros hipergrafos. Além disso, a construção de $\mathcal{A}_{p, k, g}$ é indutiva em $g$. Se diferenciando nesses aspectos, mais rentemente, N. Alon, A. Kostochka, B. Reiniger, D. B. West e $\mathrm{X}$. Zhu [4] desenvolveram uma construção para um hipergrafo $\mathcal{T}_{p, k, g} \in \mathcal{G}_{p, k, g}$ que não envolve outros hipergrafos além daquele buscado e que não é indutiva. Essa construção se baseia, porém, em uma família de grafos auxiliares que será construída indutivamente, os $(d, r, g)$-grafos.

Neste capítulo, primeiro são construídos os $(d, r, g)$-grafos. Depois, vê-se como obter $\mathcal{T}_{p, k, g}$ a partir de um certo $(d, r, g)$-grafo. A contagem do número de vértices de $\mathcal{T}_{p, k, g}$ é mais trabalhosa que as contagens anteriores. Para a cota inferior, feita por N. Alon [3], é definida uma outra família de grafos, os $T B(x, r, g)$-grafos. O número de vértices de um $T B(x, r, g)$-grafo será mais fácil de ser estimado. Um $(d, r, g)$-grafo sempre conterá um certo $T B(x, r, g)$-grafo. Assim, se for conhecida uma cota inferior para o número de vértices de um $T B(x, r, g)$-grafo, ela poderá ser utilizada para limitar inferiormente o número de vértices de um $(d, r, g)$-grafo.

\subsection{A construção dos $(d, r, g)$-grafos}

A construção de $\mathcal{T}_{p, k, g}$ utiliza uma família de grafos auxiliares, os $(d, r, g)$-grafos. Esses grafos são árvores aumentadas. Assim, é preciso definir algumas noções sobre árvores para, em seguida, dizer o que são árvores aumentadas e quais são os $(d, r, g)$-grafos.

Definição 6.1.1. Seja $T$ uma árvore com raiz. Tem-se as seguintes definições.

- O conjunto das folhas de $T$ é denotado $F(T)$.

- Seja $d \geq 1$. A árvore $T$ é $d$-ária quando todos seus vértices, exceto as folhas, têm $d$ filhos.

- Se $T$ é uma árvore $d$-ária, ela é completa quando todas as folhas estão a uma mesma distância da raiz.

- Um caminho completo em $T$ é um caminho que liga a raiz a uma folha. 
- A altura de $T$ é o número de arestas de um caminho completo mais longo.

- Se $v$ é um vértice de $T$, seus ancestrais são os vértices do caminho da raiz até $v$.

Definição 6.1.2. Seja $T$ uma árvore. Uma aresta de retorno é um par $\{v, w\}$, onde $v$ é uma folha de $T$ e $w$ é um de seus ancestrais, exceto seu pai.

Definição 6.1.3. Seja $r \geq 1$. O grafo $G$ é uma árvore $r$-aumentada quando é constituído por uma árvore $T$ mais $r$ arestas de retorno para cada folha $v \in F(T)$.

Define-se a família dos $(d, r, g)$-grafos.

Definição 6.1.4. Sejam $d \geq 2, r \geq 1$ e $g \geq 4$ inteiros. Uma árvore $r$-aumentada $G$ é um $(d, r, g)$ grafo, quando a árvore $T$ que a constitui é $d$-ária completa e, além disso, tem-se que $\operatorname{cin}(G) \geq g$. Será dito que $G$ é um $(d, r, g)$-grafo com árvore $T$ ou que $T$ é a árvore do $(d, r, g)$-grafo $G$.

Para cada $d \geq 2, r \geq 1$ e $g \geq 4$, será construído um $(d, r, g)$-grafo. A construção que será apresentada é indutiva em $g$. No passo da indução em $g$, será feita outra indução, em $r$. Isto é, os $(d, r, g)$-grafos serão construídos a partir de $g=4$ em diante, para todos $d$ e $r$. Para $g=4$, todos valores de $d$ e $r$ serão construídos ao mesmo tempo. Para cada $g>4$, serão construídos de $r=1 \mathrm{em}$ diante, para todo $d$. Para $r=1$ e qualquer valor de $d$, utiliza-se um grafo com $g$ menor. Para $r>1$ e qualquer valor de $d$, utilizam-se grafos com mesmo $g$, mas $r$ menor.

Para avaliar ostamanhos dos $(d, r, g)$-grafos, tem-se a seguinte definição.

Definição 6.1.5. Sejam $d \geq 2, r \geq 1$ e $g \geq 4$ inteiros. Defina $m(d, r, g)$ como a menor altura da árvore de um $(d, r, g)$-grafo.

Como o número de vértices de um $(d, r, g)$-grafo é igual ao número de vértices de sua árvore, tem-se que o valor de $m(d, r, g)$ é suficiente para se obter o número mínimo de vértices de um $(d, r, g)$ grafo.

Apresentar uma construção para um $(d, r, g)$-grafo é uma forma de se obter um limite superior para $m(d, r, g)$. A partir da construção que segue, na Seção 6.3, será obtida uma cota superior explícita para $m(d, r, g)$.

Teorema 6.1.1. Sejam $d \geq 2, r \geq 1$ e $g \geq 4$ inteiros. Existe um $(d, r, g)$-grafo $e$

1. $m(d, r, 4)=2 r+1$,

2. $m(d, 1, g+1) \leq m(d, d, g)+1$,

3. $m(d, r+1, g+1) \leq m_{1}+m_{2}-1$, onde $m_{1}=m(d, 1, g+1)$ e $m_{2}=m\left(d^{m_{1}}, r, g+1\right)$.

Demonstração. É dada uma construção indutiva em $g$. Para $g=4$, para quaisquer $d$ e $r$, tome uma árvore $d$-ária completa de altura $2 r+1$ e envie, a partir de cada folha, $r$ arestas de retorno que incidam em todos ancestrais à distância ímpar dela, desde 3 até a distância $2 r+1$ (Figura 6.1). Essa árvore $r$-aumentada não tem circuito de tamanho 3. De fato, é um grafo bipartido, onde uma parte contém os vértices à distância par da raiz e a outra contém os vértices à distância ímpar. Além disso, $2 r+1$ é a menor altura que a árvore de um $(d, r, 4)$-grafo pode ter, porque dois ancestrais consecutivos não podem ambos receber arestas de retorno sem formar triângulo. Assim, tem-se $m(d, r, 4)=2 r+1$. 


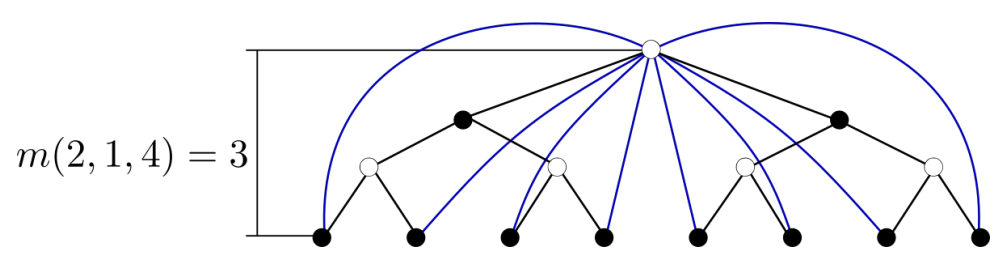

Figura 6.1: Construção de um $(2,1,4)$-grafo.

Seja $g \geq 4$ e considere que para todo $d \geq 2$ e $r \geq 1$, existe um $(d, r, g)$-grafo. Dados $r$ e $d$, quer-se construir um $(d, r, g+1)$-grafo. Isso será feito por indução em $r$. Para construir um $(d, 1, g+1)$-grafo, tome um $(d, d, g)$-grafo $G^{\prime}$, dado pela hipótese da indução em $g$, e seja $T^{\prime}$ sua árvore. A cada folha $v$ de $T^{\prime}$, acrescente $d$ filhos $v_{i}(i \in[d])$; essa árvore será $T$. Para cada uma das arestas de retorno de $G^{\prime}$ da forma $v x_{i}(i \in[d])$, adicione uma aresta de retorno em $T$ da forma $v_{i} x_{i}$, de modo que cada $v_{i}$ receba exatamente uma aresta de retorno (Figura 6.2). Chame esse grafo de G. Prova-se que ele é um $(d, 1, g+1)$-grafo.

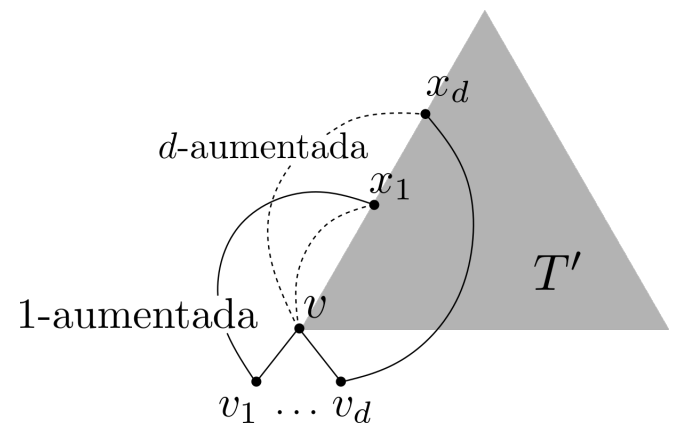

Figura 6.2: Construção de um $(d, 1, g+1)$-grafo a partir de um $(d, d, g)$-grafo.

Suponha por contradição que $G$ possui um circuito $C$ de comprimento menor que ou igual a $g$. A partir de $C$, será encontrado um circuito em $G^{\prime}$. O circuito $C$ deve conter pelo menos uma aresta de retorno $v_{i} x_{i}$. Cada folha $v_{i}$ de $T$ tem grau 2, então o circuito contém o segmento $\left(v, v_{i}, x_{i}\right)$, onde $v$ é o pai de $v_{i}$. Substitua esse segmento pela aresta de retorno $v x_{i}$ de $G^{\prime}$. Para toda aresta de retorno do circuito $C$, faça essa substituição. Chame de $C^{\prime}$ a sequência resultante.

Afirma-se que $C^{\prime}$ é um circuito em $G^{\prime}$. Primeiro note que todos vértices de $C^{\prime}$ são vértices de $G^{\prime}$, pois todos $v_{i} \in V(G) \backslash V\left(G^{\prime}\right)$ em $C$ fazem parte de uma aresta de retorno de $C$. Ao se fazer a substituição de $\left(v, v_{i}, x_{i}\right)$ por $v x_{i}$, tem-se que $v_{i}$ é removido.

Depois note que cada par de vértices consecutivos de $C^{\prime}$ está conectado por uma aresta de $G^{\prime}$. As únicas arestas em $E(G) \backslash E\left(G^{\prime}\right)$ são as que incidem em algum $v_{i} \in V(G) \backslash V\left(G^{\prime}\right)$. Sejam $x$ e $y$ dois vértices consecutivos em $C^{\prime}$. Como dito, tem-se que $x$ e $y$ são vértices de $G^{\prime}$. Assim, se $x$ e $y$ também forem consecutivos em $C$, tem-se que a aresta que os conecta em $C$ é também uma aresta de $G^{\prime}$. Se $x$ e $y$ não forem consecutivos em $C$, eles são extremos de um segmento $\left(v, v_{i}, x_{i}\right)$ que foi substituído por $v x_{i}$. Por construção, $v x_{i}$ é uma aresta de retorno em $G^{\prime}$.

Além disso, afirma-se que $C^{\prime}$ tem pelo menos 3 arestas. Existem duas possíveis continuações em $C$ para o segmento $\left(v, v_{i}, x_{i}\right)$. A primeira é $\left(w, v, v_{i}, x_{i}\right)$, com $w$ sendo o pai de $v$ em $T^{\prime}$. Tem-se que $w \neq x_{i}$, pois nenhuma aresta de retorno de $v$ em $T^{\prime}$ atinge o pai de $v$. Nesse caso, $C^{\prime}$ conterá pelo menos $\left(w, v, x_{i}\right)$. A segunda continuação possível é $\left(x_{j}, v_{j}, v, v_{i}, x_{i}\right)$. Tem-se que $x_{j} \neq x_{i}$, pois $v$ envia $r$ arestas de retorno em $T^{\prime}$, portanto $r$ ancestrais distintos são atingidos. Cada $v_{i}$ foi ligado a um desses ancestrais distintos. Nesse caso, $C^{\prime}$ conterá pelo menos $\left(x_{j}, v, x_{i}\right)$. 
Por fim, afirma-se que nenhum vértice de $C^{\prime}$ é repetido. De fato, apenas foram apagados alguns vértices de $C$, que é circuito e portanto não possui vértices repetidos. Tem-se que $C^{\prime}$ é um circuito em $G^{\prime}$.

O grafo $G^{\prime}$ tem cintura pelo menos $g$, mas o comprimento de $C^{\prime}$ é menor que ou igual a $g-1$; uma contradição. Tem-se assim que $G$ é um $(d, 1, g+1)$-grafo e que $m(d, 1, g+1) \leq m(d, d, g)+1$.

Seja $r \geq 1$ e suponha, para todo $d \geq 2$, que existe um $(d, r, g+1)$-grafo. Para o passo de indução, quer-se construir um $(d, r+1, g+1)$-grafo. Serão utilizados um $(d, 1, g+1)$-grafo $G_{1}$ cuja árvore $T_{1}$ tenha altura mínima $m(d, 1, g+1)=m_{1}$, e um $\left(d^{m_{1}}, r, g+1\right)$-grafo $G_{2}$, dado pela hipótese da indução em $r$, cuja árvore $T_{2}$ tenha altura $m\left(d^{m_{1}}, r, g+1\right)=m_{2}$.

Os grafos $G_{1}$ e $G_{2}$ serão combinados para se obter um $(d, r+1, g+1)$-grafo $G$. Antes de apresentar esse processo precisamente, descreve-se em linhas gerais o que será feito. Para cada vértice em $T_{2}$ que seja pai de folhas, ele e todos seus $d^{m_{1}}$ filhos serão substituídos por uma cópia de $T_{1}$, obtendo-se uma nova árvore $T^{\prime}$. O número de folhas de $T_{1}$ é $d^{m_{1}}$. Assim, cada folha de $T_{2}$ pode ser associada a alguma folha da cópia de $T_{1}$ que entrou em seu lugar. Dessa forma, as $r$ arestas de retorno em $G_{2}$ de cada folha de $T_{2}$ definem novas arestas de retorno com extremo na folha associada da cópia de $T_{1}$. Adicionando-se também as arestas de retorno de $G_{1}$ em cada cópia de $T_{1}$, obtém-se uma árvore $(r+1)$-aumentada, mas que ainda não é um $(d, r+1, g+1)$-grafo (Figura 6.4).

Para se obter um $(d, r+1, g+1)$-grafo, é preciso garantir que todos vértices internos de sua árvore tenham $d$ filhos. Isso não ocorre em $T^{\prime}$, pois os vértices internos de $T_{2}$ têm $d^{m_{1}}$ filhos. Por isso, antes de fazer a substituição das folhas em $T_{2}$, é tomado um subgrafo $T_{H}$ dele. Nesse subgrafo, todos os vértices internos devem ter $d$ filhos, exceto os pais de folhas, que continuam com seus $d^{m_{1}}$ filhos (Figura 6.3). A substituição das folhas por cópias de $T_{1}$ será feita em $T_{H}$, obtendo-se a árvore $T$. As arestas de retorno serão adicionadas da mesma forma, obtendo-se uma árvore $(r+1)$-aumentada $G$. Será provado que $\operatorname{cin}(G) \geq g+1$, de modo que $G$ é um $(d, r+1, g+1)$-grafo.

Como foi dito, agora a construção é apresentada mais formalmente. Inicia-se tomando um subgrafo $T_{H}$ de $T_{2}$. Construa $T_{H}$ da raiz para as folhas. Escolha a raiz $r_{0}$ de $T_{2}$ para ser um vértice de $T_{H}$. Coloque $r_{0}$ numa fila. Considere o primeiro vértice $v$ na fila. Escolha $d$ de seus $d^{m_{1}}$ filhos em $T_{2}$ para serem vértices de $T_{H}$; sejam eles $v_{1}, \ldots, v_{d}$. Retire $v$ do início da fila. Se $v_{1}, \ldots, v_{d}$ não forem pais de folhas em $T_{2}$, coloque-os no fim da fila. Senão, inclua também em $T_{H}$, para cada $v_{i}$, todos seus $d^{m_{1}}$ filhos em $T_{2}$ e não acrescente nenhum vértice à fila. O processo termina quando a fila está vazia. No final, tem-se que $T_{H}$ é uma árvore $d$-ária exceto pelos pais das folhas, que têm $d^{m_{1}}$ filhos.

Como, para cada folha $v$ de $T_{H}$, é tomado todo o caminho desde a raiz até $v$, todos seus ancestrais em $T_{2}$ também estão em $T_{H}$. Assim, pode-se adicionar a cada folha de $T_{H}$ as $r$ arestas de retorno que incidiam sobre ela em $G_{2}$. Obtém-se o grafo $H$, que é um subgrafo induzido de $G_{2}$ (Figura 6.3).

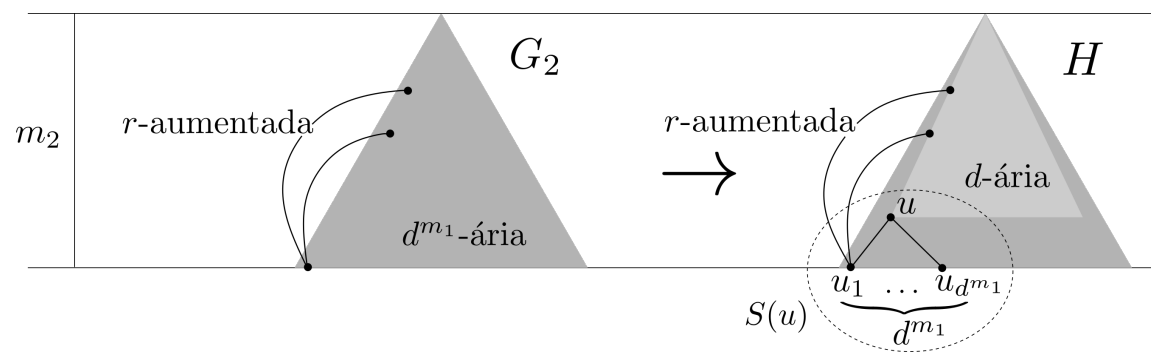

Figura 6.3: Construção do subgrafo $H$ a partir de um $\left(d^{m_{1}}, r, g+1\right)$-grafo. 
Considere um vértice $u$ que seja pai das folhas $u_{i}\left(i \in\left[d^{m_{1}}\right]\right)$ em $T_{H}$. Seja $S(u)$ a estrela composta por $u$ e seus filhos. Substitua $S(u)$ por uma cópia de $T_{1}$, que será chamada de $T_{1}(u)$, da seguinte forma. Seja $x$ o pai de $u$ em $T_{H}$. Remova $S(u)$ e conecte $x$ com a raiz $r_{1}(u)$ de $T_{1}(u)$. Faça essa substituição para cada vértice que é pai de folhas em $T_{H}$ e obtenha a árvore $T$, que é $d$-ária e completa.

Para cada folha de $T$ serão adicionadas $r+1$ arestas de retorno. Considere uma cópia $T_{1}(u)$. Primeiro acrescente a cada folha de $T_{1}(u)$ uma aresta de retorno, de modo a obter uma cópia de $G_{1}$, que será chamada de $G_{1}(u)$. Depois adicione mais $r$ arestas de retorno para cada folha de $T_{1}(u)$ da seguinte forma. Associe a folha $v_{i}\left(i \in\left[d^{m_{1}}\right]\right)$ de $T_{1}(u)$ ao filho $u_{i}\left(i \in\left[d^{m_{1}}\right]\right)$ de $u$ em $T_{H}$. Adicione arestas de retorno em $T$ que ligam $v_{i}$ aos mesmos $r$ ancestrais aos quais $u_{i}$ está ligado em $H$ (Figura 6.4). Faça esse procedimento para toda cópia $T_{1}(u)$ em $T$. Obtém-se o grafo $G$. Ele é uma árvore $(r+1)$-aumentada com árvore $T$ (Figura 6.4). Chame as arestas de retorno em $G$ que estão contidas em cópias de $G_{1}$ de arestas curtas e chame as que ligam as folhas a vértices de $H$ de arestas longas.

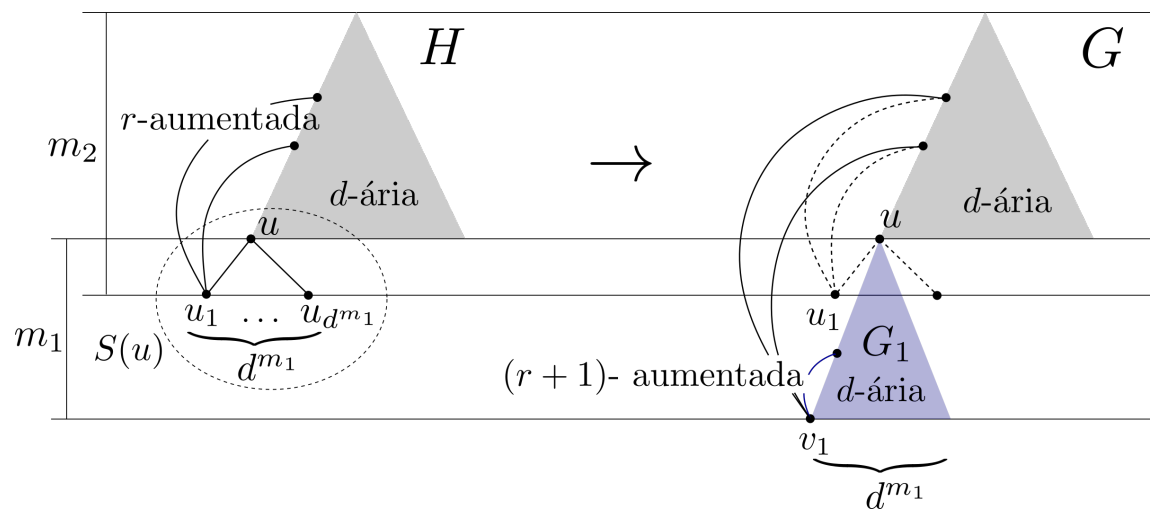

Figura 6.4: Construção de um $(d, r+1, g+1)$-grafo a partir de $H \subset G_{2}$ e cópias de $G_{1}$.

Para $G$ ser um $(d, r+1, g+1)$-grafo, basta provar que não há circuito de comprimento menor que ou igual a $g$. Suponha por contradição que existe um circuito $C$ em $G$ com comprimento no máximo $g$. Se $C$ não usa aresta de retorno longa, deve usar alguma aresta curta $x y$. Suponha que $x y$ está na cópia $G_{1}(u)$ e que $y$ não é a raiz de $T_{1}(u)$. A partir de $x$, o circuito $C$ percorre um caminho até atingir $y$ e se fechar. Se esse caminho em $C$ sai de $G_{1}(u)$ sem usar arestas longas, então ele passa pela raiz de $T_{1}(u)$. Porém é preciso retornar a $y$, que está em $G_{1}(u)$. Não é possível retornar a $G_{1}(u)$ sem usar arestas longas nem passar novamente pela raiz de $T_{1}(u)$. Assim, tem-se que $C$ está contido em $G_{1}(u)$. Como $\operatorname{cin}\left(G_{1}\right) \geq g+1$, o comprimento de $C$ é pelo menos $g+1$, uma contradição.

Tem-se que $C$ contém pelo menos uma aresta longa, de modo que pelo menos um vértice de $C$ é de $H$ e pelo menos um é de uma cópia $G_{1}\left(u_{0}\right)$. As cópias de $G_{1}$ serão contraídas de volta às estrelas e $C$ irá determinar um circuito em $G_{2}$ (Figura 6.5). Considere que $C$ contém um segmento na cópia $G_{1}(u)$. Se o segmento é apenas um vértice, ele deve ser uma folha $v_{i}$ de $T_{1}(u)$. Substitua $v_{i}$ por $u_{i}$. Se o segmento contém mais vértices, ele deve ter como extremos a raiz $r_{1}(u)$ e uma folha ou duas folhas distintas de $T_{1}(u)$, pois são as únicas formas de entrar em ou sair de $G_{1}(u)$. Se tiver como extremos $r_{1}(u)$ e uma folha $v_{i}$, ele é contraído à aresta $u u_{i}$ em $S(u) \subset H \subset G_{2}$. Se tiver como extremos as folhas $v_{i} \neq v_{j}$, ele é contraído ao caminho $\left(u_{i}, u, u_{j}\right)$ em $S(u) \subset H \subset G_{2}$.

Os extremos do segmento mudam. Afirma-se que após a substituição, o segmento passa a se 
conectar com o resto de $C$ por arestas de $G_{2}$. Suponha que a raiz $r_{1}(u)$ é um dos extremos do segmento que será substituído. Pela forma que $G$ é construído, o único vizinho de $r_{1}(u)$ que não está em $G_{1}(u)$ é o pai de $u$ em $T_{H}$, que é o vértice $x$. Assim, esse segmento de $C$ com extremo $r_{1}(u)$ se conecta ao resto de $C$ pela aresta $r_{1}(u) x$. Após a substituição, tem-se automaticamente que $u x$ é aresta de $T_{H} \in H \in G_{2}$. Quando o extremo do segmento de $C$ em $G_{1}(u)$ é uma folha $v_{i}$ de $T_{1}(u)$, tem-se que $v_{i}$ se conecta ao resto do circuito por uma aresta longa $v_{i} x_{i}$. Pela construção de $G$, as arestas longas com um dos extremos em $v_{i}$ o conectam aos mesmos ancestrais aos quais $u_{i}$ se conecta em $H$. Portanto, $u_{i} x_{i}$ é uma aresta de $H \in G_{2}$.

Substituindo-se todos os segmentos de $C$ em alguma cópia de $G_{1}$, obtém-se uma sequência de vértices $C^{\prime}$ cujos vértices consecutivos estão ligados por arestas em $G_{2}$, considerando que o primeiro vértice é consecutivo ao último. $\mathrm{O}$ comprimento do segmento contraído é menor que ou igual ao comprimento do segmento original, assim $\left|C^{\prime}\right| \leq|C|$.
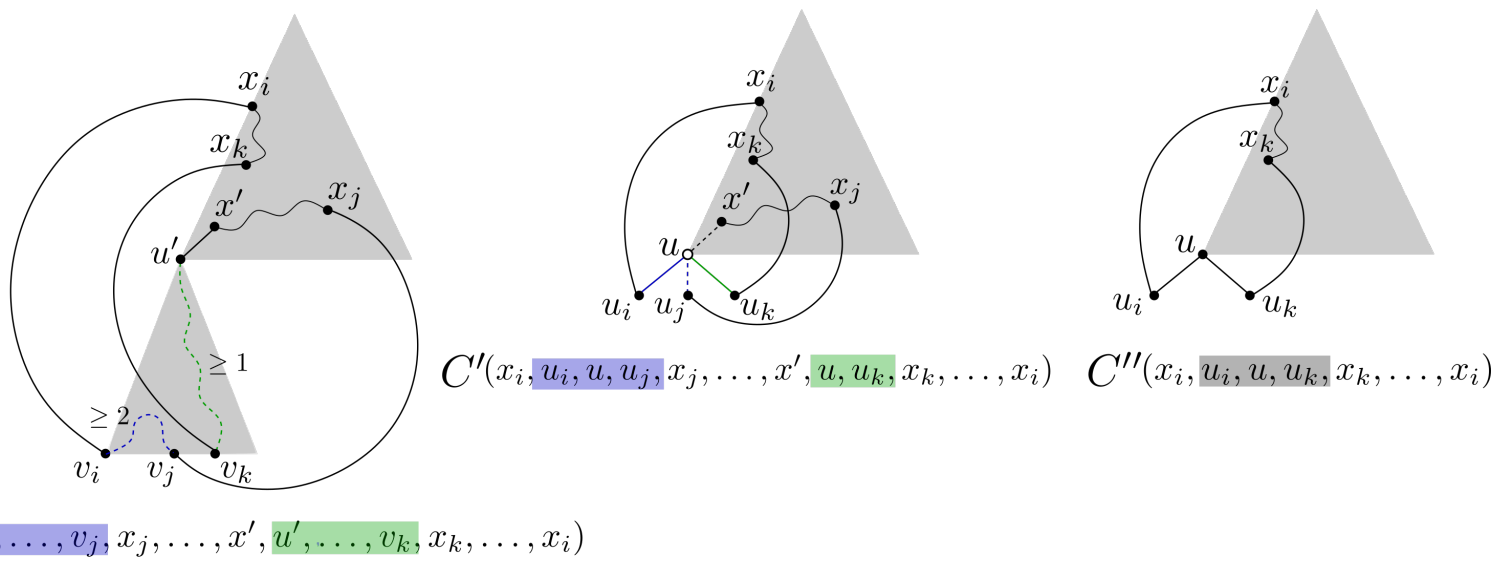

$C\left(x_{i}, v_{i}, \ldots, v_{j}, x_{j}, \ldots, x^{\prime}, u^{\prime}, \ldots, v_{k}, x_{k}, \ldots, x_{i}\right)$

Figura 6.5: Contraindo as cópias de $G_{1}$ de volta às estrelas e o circuito em $G_{2}$.

Se existir mais de um segmento em $C$ numa mesma cópia $G_{1}(u)$, pode ser que $u$ se repita em $C^{\prime}$, de modo que $C^{\prime}$ ainda não seja um circuito. Serão feitas outras contrações em $C^{\prime}$ para se obter um circuito $C^{\prime \prime}$ em $G_{2}$. Pode-se escrever a sequência de vértices $C^{\prime}$ a partir de qualquer um de seus vértices e isso não muda o fato de vértices consecutivos estarem conectados por arestas de $G_{2}$. Assim, inicie a sequência por um vértice de $H$; já foi notado que há um tal vértice em $C$ e, portanto, também em $C^{\prime}$. Para cada estrela $S(u)$, tome o primeiro vértice $x$ e o último vértice $y$ em $C^{\prime}$ pertencentes à estrela $S(u)$. Todo segmento entre $x$ e $y$ será contraído. Os vértices $x$ e $y$ podem ser $u$ e algum de seus filhos $u_{i}$ e, nesse caso, o segmento é contraído à aresta $u u_{i}$. Ou $x$ e $y$ podem ser duas folhas $u_{i} \neq u_{j}$, caso em que tudo é contraído a $\left(u_{i}, u, u_{j}\right)$. Se $u_{i}=u_{j}$, o segmento é apenas um vértice e mantém-se $u_{i}$. Depois dessa contração, considere outra estrela e faça o mesmo procedimento. Quando todas estrelas tiverem sido consideradas, tem-se a sequência $C^{\prime \prime}$. Em $C^{\prime \prime}$, não haverá mais repetição de vértices e dois vértices consecutivos sempre estarão conectados por arestas de $G_{2}$, pois os extremos não são alterados. Além disso, afirma-se que haverá pelo menos três vértices em $C^{\prime \prime}$. De fato, pela forma que $C^{\prime}$ é reescrito antes de se fazer as contrações e como as contrações não alteram os extremos, tem-se que o primeiro vértice de $C^{\prime}$, o segundo e o último são mantidos em $C^{\prime \prime}$. Dessa forma, $C^{\prime \prime}$ é um circuito em $G_{2}$ e $\left|C^{\prime \prime}\right| \leq\left|C^{\prime}\right| \leq|C| \leq g$. Mas $G_{2}$ tem cintura pelo menos $g+1$, contradição. Conclui-se que $G$ é um $(d, r+1, g+1)$-grafo. Como a altura de $T$ é $m_{1}+m_{2}-1$, tem-se que $m_{1}+m_{2}-1 \geq m(d, r+1, g+1)$. 


\subsection{A construção dos $\mathcal{T}_{p, k, g}$}

Na construção de $\mathcal{T}_{p, k, g}$, será utilizado um certo $\left(d, r, g^{\prime}\right)$-grafo $G$ com árvore $T$. O parâmetro $g^{\prime}$ dependerá de $g$ e será o que garantirá a cintura grande. O parâmetro $d$ dependerá de $k$, e $r$ dependerá de $p$ e $k$; são eles que garantirão o número cromático grande e a $p$-uniformidade. No que se segue, discute-se como definir esses parâmetros.

Começar com uma árvore $T$ e adicionar arestas de retorno é uma boa forma de controlar a cintura do grafo. Porém o número cromático de uma árvore aumentada é no máximo 3, basta colorir os vértices internos da árvore com duas cores e as folhas com uma terceira cor. Uma árvore aumentada não poderia ser um grafo em $\mathcal{G}_{p, k, g}$, mas é possível construir um $\mathcal{T}_{p, k, g} \in \mathcal{G}_{p, k, g}$ sobre seus vértices.

Assim, tome um $\left(d, r, g^{\prime}\right)$-grafo $G$ com árvore $T$. A ideia para garantir que $\mathcal{T}_{p, k, g}$ tenha cintura grande é fazer um circuito em $\mathcal{T}_{p, k, g}$ induzir um circuito em $G$. Uma forma de conseguir isso é fazendo cada aresta de $\mathcal{T}_{p, k, g}$ corresponder a um caminho de tamanho 2 em $G$. Esses caminhos devem ser tais que, substituindo-se as arestas de um circuito em $\mathcal{T}_{p, k, g}$ por eles, obtém-se um circuito em $G$. Dessa forma, um circuito de tamanho $\ell$ em $\mathcal{T}_{p, k, g}$ corresponderá a um circuito de tamanho $2 \ell$ em $G$. Tem-se o motivo para tomar $g^{\prime}=2 g+1$.

Para se conseguir que cada aresta $\mathcal{T}_{p, k, g}$ corresponda a um tal caminho em $G$, as arestas de $\mathcal{T}_{p, k, g}$ são definidas a partir de estrelas contidas em $G$. Todos os vértices de uma aresta de $\mathcal{T}_{p, k, g}$ estarão conectados, em $G$, a um outro vértice fora dessa aresta, formando uma estrela em $G$. Dada uma aresta de $\mathcal{T}_{p, k, g}$, o vértice central da estrela que a define será o vértice central do caminho correspondente a ela. Se os vértices centrais de estrelas distintas forem distintos e se eles não estiverem contidos em nenhuma aresta de $\mathcal{T}_{p, k, g}$, então fica garantido que a substituição das arestas do circuito pelos caminhos de tamanho 2 correspondentes forma um circuito em $G$. Cada estrela de $G$ que será utilizada para definir uma aresta de $\mathcal{T}_{p, k, g}$ terá como vértice central uma folha de $F(T)$ e seus vértices de grau 1 serão $p$ ancestrais dessa folha atingidos por arestas de retorno. Assim, as arestas de $\mathcal{T}_{p, k, g}$ serão $e_{v}(v \in F(T))$, onde $e_{v}$ é constituído por $p$ vértices ligados a $v$ por arestas de retorno de $G$ (Figura 6.6).
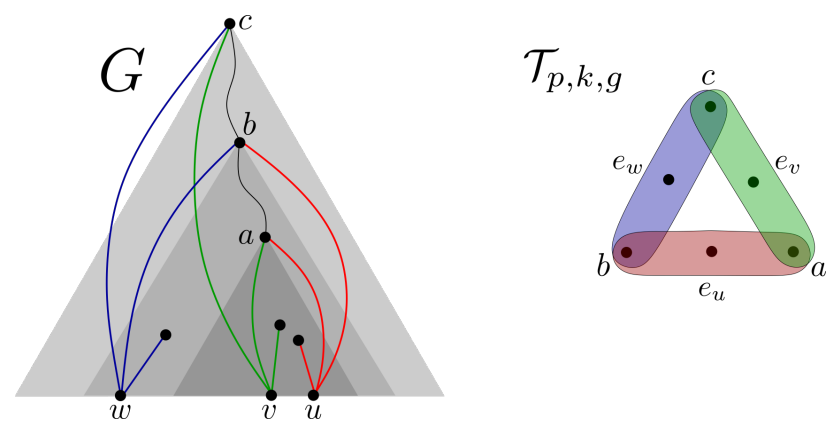

Figura 6.6: Um circuito de $\mathcal{T}_{p, k, g}$ corresponde a um circuito em $G$.

Ainda é preciso garantir número cromático maior que $k-1$. Pela forma que serão definidas as arestas de $\mathcal{T}_{p, k, g}$, tem-se que os vértices de $\mathcal{T}_{p, k, g}$ serão os vértices de $T$, exceto $F(T)$. Uma coloração com cores em $[k-1]$ dos vértices de $\mathcal{T}_{p, k, g}$ é uma coloração dos vértices internos de $T$. A cada folha, pode-se associar a sequência que corresponde às cores dos vértices no caminho da raiz até essa folha. Essa sequência tem termos em $[k-1]$ e tamanho igual à altura da árvore. Se $T$ for uma árvore $(k-1)$-ária, há ainda outra forma de se associar a uma folha uma sequência com termos 
em $[k-1]$ e tamanho igual à altura da árvore. Enumere as arestas que ligam cada vértice interno a seus filhos de 1 a $k-1$. A sequência correspondente a uma folha nesse caso será formada pelos números das arestas do caminho completo até ela, a partir da aresta que incide na raiz até a que incide na folha. Para cada folha, ficam associadas duas sequências. A relação entre essas sequências que será demonstrada é que sempre existirá uma folha $v_{0}$ para a qual as duas sequências associadas são iguais. Assim, no caminho completo até $v_{0}$, a cor de cada vértice é igual ao número da aresta que desce dele para seu filho no caminho (Figura 6.7). Isso significa que uma única enumeração das arestas carrega um pouco de informação sobre cada uma das possíveis colorações dos vértices. Dada uma coloração dos vértices, em algum caminho completo, ela estará refletida pela enumeração das arestas. Tem-se o motivo para tomar $d=k-1$.

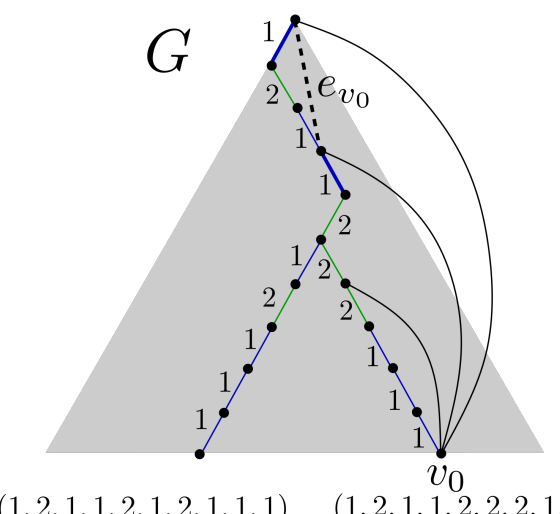

$(1,2,1,1,2,1,2,1,1,1)$

$(1,2,1,1,2,2,2,1,1,1)$

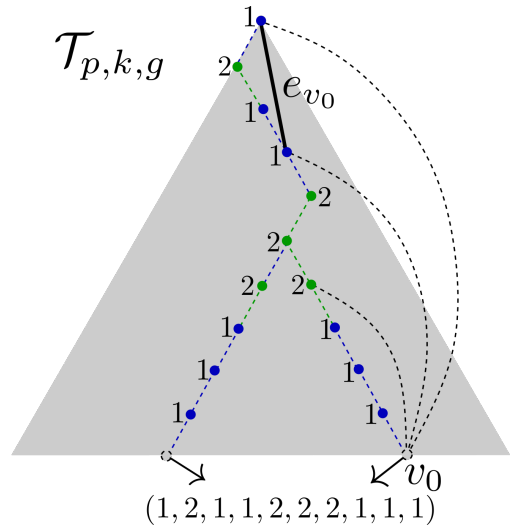

Figura 6.7: A folha $v_{0}$ está associada à mesma sequência tanto para a enumeração das arestas, quanto para a coloração dos vértices.

Fixe uma enumeração das arestas da árvore. Essa enumeração será observada para determinar quais $p$ vértices serão incluídos em cada aresta $e_{v}$, garantindo que $k-1$ cores sempre deixem uma aresta monocromática em $\mathcal{T}_{p, k, g}$. Como dito, cada aresta $e_{v}$ será composta por vizinhos de $v$ atingidos por arestas de retorno. Assim, para definir $e_{v}$, observam-se apenas os números das arestas que ligam cada um desses vizinhos de $v$ a seu filho no caminho completo. Se o número de arestas de retorno que partem de $v$ for $(p-1)(k-1)+1$, pode-se garantir que $p$ das arestas observadas têm mesmo número. Esses $p$ vizinhos de $v$ formarão $e_{v}$. Dessa forma, ao se tomar uma coloração qualquer dos vértices, encontra-se a folha $v_{0}$ que se associa à mesma sequência tanto na enumeração das arestas quanto na coloração dos vértices. Os $p$ vizinhos de $v_{0}$ que compõem $e_{v_{0}}$ se ligam a seus filhos no caminho completo até $v_{0}$ por arestas de mesmo número, por construção. Como as sequências coincidem, tem-se que $e_{v_{0}}$ será a monocromática. Por isso, será tomado $r=(p-1)(k-1)+1$.

Dada a ideia, prova-se formalmente o seguinte teorema.

Teorema 6.2.1. Dados $p \geq 2, k \geq 3$ e $g \geq 2$, existe hipergrafo $\mathcal{T}_{p, k, g} \in \mathcal{G}_{p, k, g}$. Esse $\mathcal{T}_{p, k, g}$ é obtido a partir de um $(k-1,(p-1)(k-1)+1,2 g+1)$-grafo.

Demonstração. Sejam $p \geq 2, k \geq 3$ e $g \geq 2$ inteiros. Tome $G$ um $(k-1,(p-1)(k-1)+1,2 g+1)$ grafo com árvore $T$. A árvore $T$ é $(k-1)$-ária completa. Fixe uma coloração $\phi$ das arestas de $T$ de forma que, para cada vértice interno, as arestas que o ligam a seus filhos recebam cada uma, uma cor distinta de $[k-1]$. Essa coloração $\phi$ é a enumeração das arestas sobre a qual se falou anteriormente, para justificar o motivo de se tomar $d=k-1$. Mais adiante, será provado que ela carrega informação sobre cada uma das possíveis colorações dos vértices internos de $T$. Primeiro, 
utiliza-se $\phi$ para definir as arestas de $\mathcal{T}_{p, k, g}$.

Seja $v$ uma folha de $T$ e seja $P_{v}$ o caminho completo em $T$, da raiz até $v$. Como $G$ é uma árvore $((p-1)(k-1)+1)$-aumentada, tem-se que $(p-1)(k-1)+1$ vértices de $P_{v}$ estão ligados a $v$. Considere as arestas em $P_{v}$ entre cada um dos ancestrais ligados a $v$ e seu filho no caminho; são $(p-1)(k-1)+1$ arestas. Como essas arestas receberam cores em $[k-1]$, existem pelo menos $p$ delas que receberam a mesma cor; elas são $x_{i} y_{i}(i \in[p])$, com $x_{i}$ pai de $y_{i}$ e com $x_{i}$ adjacente a $v$. Para cada folha $v$, defina o conjunto $e_{v}=\left\{x_{i}: i \in[p]\right\}$ (Figura 6.8). Tem-se o hipergrafo $\mathcal{T}_{p, k, g}$, com $V\left(\mathcal{T}_{p, k, g}\right)=V(T)-F(T)$ e $E\left(\mathcal{T}_{p, k, g}\right)=\left\{e_{v}: v \in F(T)\right\}$. Será visto que $\mathcal{T}_{p, k, g}$ é um $\mathcal{G}_{p, k, g}$. Note que $\mathcal{T}_{p, k, g}$ é $p$-uniforme, pois cada conjunto $e_{v}$ tem tamanho $p$.
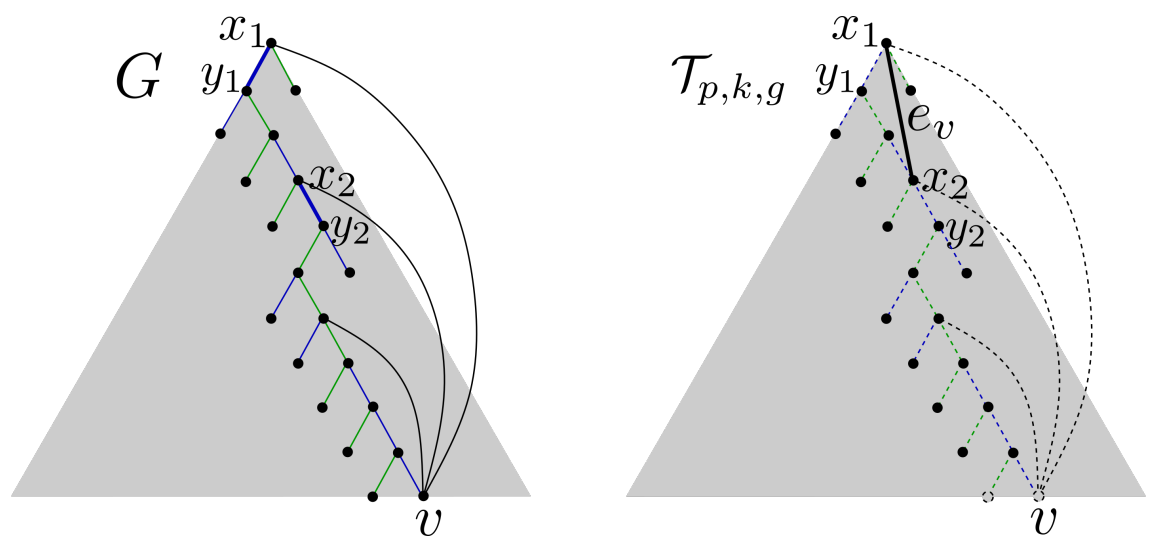

Figura 6.8: Uma representação para o caso $p=2, k=3, g=2$, em que $G$ é um $(2,3,5)$-grafo e $\phi$ é uma 2 -coloração das arestas. Mostra-se como obter a aresta $e_{v}$ de $\mathcal{T}_{2,3,5}$.

Para checar que $\chi\left(\mathcal{T}_{p, k, g}\right) \geq k$, tome uma coloração $f$ de $V\left(\mathcal{T}_{p, k, g}\right)$ com cores em [k-1]. A coloração $f$ é uma coloração dos vértices internos de $T$. Lembre-se da coloração $\phi$ das arestas de $T$; a forma pela qual ela fornece informação sobre $f$ é a seguinte. Afirma-se que existe um caminho completo $P_{v_{0}}$ em $T$ tal que, da raiz até sua folha $v_{0}$, a sequência de cores de seus vértices é igual à sequência de cores de suas arestas. Isso é o mesmo que dizer que se um vértice $x$ nesse caminho tem cor $f(x)=c$, então a aresta em $P_{v_{0}}$ entre $x$ e seu filho $y$ no caminho deve ter cor $\phi(x y)=c$ também. Um caminho completo com essa propriedade é chamado de $f$-caminho. Na Figura 6.7, o caminho completo da raiz a $v_{0}$ é um $f$-caminho.

Prova-se que, para toda coloração $f$, sempre existirá um $f$-caminho e ele será único. Para encontrar esse $f$-caminho, observe que é necessário começar pela raiz $r$, tomar a sua cor $f(r)$ e observar qual filho de $r$ é atingido pela aresta de cor $f(r)$. Esse filho existe e é único, pois na coloração $\phi$, cada uma das $k-1$ arestas entre um vértice interno e seus filhos recebeu uma cor distinta em $[k-1]$. Se $x$ é o filho de $r$ tal que $\phi(r x)=f(r)$, considera-se, agora, a subárvore com raiz $x$ e o processo da construção do $f$-caminho continua até uma folha ser atingida e obter-se um caminho completo.

Para a coloração $f$ que foi tomada, encontre o $f$-caminho em $T$ e seja $v_{0}$ sua folha. Assim, o caminho completo $P_{v_{0}}$ que desce da raiz até $v_{0}$ tem apenas arestas cuja cor, segundo $\phi$, corresponde à cor, segundo $f$, do seu extremo que é o vértice pai. Por definição, os vértices em $e_{v_{0}}$ são vértices de $P_{v_{0}}$ que são extremos que são pais em arestas de $P_{v_{0}}$ que receberam a mesma cor segundo a coloração $\phi$. Dessa forma, os vértices em $e_{v_{0}}$ também receberam a mesma cor segundo $f$. Portanto, a aresta $e_{v_{0}}$ é monocromática.

Por fim, checa-se a cintura de $\mathcal{T}_{p, k, g}$. Seja $C=\left(e_{v_{1}}, \ldots, e_{v_{\ell}}\right)$ um circuito em $\mathcal{T}_{p, k, g}$. Pela de- 
finição de circuito, existem $x_{i} \in\left(e_{v_{i}} \cap e_{v_{i+1}}\right)$, com $e_{v_{\ell+1}}=e_{v_{1}}$ e $x_{i} \neq x_{j}$, para $i \neq j \in[\ell]$. Como todo vértice em $e_{v_{i}}$ é adjacente a $v_{i}$ em $G$, tem-se que $x_{i}$ é adjacente a $v_{i}$ e $v_{i+1}$. Portanto, $C^{\prime}=\left(v_{1}, x_{1}, v_{2}, \ldots, v_{\ell}, x_{\ell}\right)$ é um circuito em $G$ de comprimento $2 \ell \geq \operatorname{cin}(G)>2 g$. Desse modo, tem-se que $\operatorname{cin}\left(\mathcal{T}_{p, k, g}\right)>g$.

\subsection{O número de vértices de $\mathcal{T}_{p, k, g}$}

O número de vértices de $\mathcal{T}_{p, k, g}$ é o número de vértices do $(k-1,(p-1)(k-1)+1,2 g+1)$-grafo utilizado em sua construção menos o número de folhas de sua árvore. Sendo $h$ a altura dessa árvore,

$$
\left|V\left(\mathcal{T}_{p, k, g}\right)\right|=\sum_{i=0}^{h-1}(k-1)^{i}=\frac{(k-1)^{h}-1}{k-2} .
$$

O número de arestas de $\mathcal{T}_{p, k, g}$ é menor ou igual ao número de folhas da árvore,

$$
\left|E\left(\mathcal{T}_{p, k, g}\right)\right| \leq(k-1)^{h}
$$

Então o número de arestas é linear no número de vértices, $\left|E\left(\mathcal{T}_{p, k, g}\right)\right| \leq(k-2)\left|V\left(\mathcal{T}_{p, k, g}\right)\right|+1$. Porém esse grafo pode conter subgrafos densos. Existe uma outra construção para $p=2$, isto é, para um grafo com cintura e número cromático grandes, também a partir de um $(d, r, g)$-grafo, que garante que o grau médio máximo, $d_{\max }=\max _{H \in G} 2 E(H) / V(H)$, seja limitado. Dessa forma, todos subgrafos serão esparsos. Essa construção pode ser vista em [4].

O que será visto aqui é que o número de vértices de $\mathcal{T}_{p, k, g}$ é muito grande. Esse número depende da altura da árvore do $(k-1,(p-1)(k-1)+1,2 g+1)$-grafo. Dessa forma, prova-se uma cota superior e uma inferior para $m(d, r, g)$.

\subsubsection{Uma cota superior para $m(d, r, g)$}

Para a cota superior, utilizam-se as relações obtidas no Teorema 6.1.1. Para $d \geq 2, r \geq 1$ e $g \geq 4$ inteiros, tem-se:

1. $m(d, r, 4)=2 r+1$,

2. $m(d, 1, g+1) \leq m(d, d, g)+1$,

3. $m(d, r+1, g+1) \leq m_{1}+m_{2}-1$, onde $m_{1}=m(d, 1, g+1)$ e $m_{2}=m\left(d^{m_{1}}, r, g+1\right)$.

Quando $g=4$, tem-se que $m(d, r, 4)$ é linear em $r$. Quando $g=5$, tem-se

$$
\begin{aligned}
& m(d, 1,5)=2+2 d \\
& m(d, 2,5) \leq 4+2 d+2 d^{2 d+2}, \\
& m(d, 3,5) \leq 6+2 d+2 d^{2 d+2}+2\left(d^{2 d+2}\right)^{2\left(d^{2 d+2}\right)+2} .
\end{aligned}
$$

Sempre aplicando a relação 3 para obter $m(d, r+1,5)$, substitui-se $d$ por $d^{2 d+2}$ em $m(d, r, 5)$. Nota-se 
que $m(d, r, 5)$ tem como fator de maior ordem algo da forma

$$
\underbrace{\left(d^{d}\right)^{\cdot{ }^{\left.\cdot d^{d}\right)}}}_{r-1} \leq \underbrace{d^{\cdot \cdot} \cdot{ }^{d}}_{2(r-1)}=\underbrace{d \uparrow(\ldots d \uparrow(d \uparrow d) \ldots)}_{2(r-1)}=d \uparrow^{2} 2(r-1) .
$$

Assim, uma possível cota superior teria forma $d \uparrow^{g} r$. O que será feito é uma indução em $g$ e, no passo dessa indução, uma indução em $r$. A relação 3 será utilizada no passo da indução em $r$. Essa relação envolve $m(d, 1, g+1)$, que é o caso base da indução em $r$, e esse valor aparece no expoente de $d$. Será preciso fazer algo parecido com o que foi feito em (6.2), onde foi notado que

$$
\underbrace{\left(d^{d}\right) \cdot \cdot^{\left.\cdot d^{d}\right)}}_{r-1}=\left(d \uparrow^{2} 2\right) \uparrow^{2}(r-1) \leq d \uparrow^{2} 2(r-1) .
$$

Em geral, tem-se o seguinte lema.

Lema 6.3.1. Sejam $a \geq 2$ e $b, c \geq 1$ e $x \geq 0$ inteiros. Vale que

$$
\left(a \uparrow^{x} b\right) \uparrow^{x} c \leq a \uparrow^{x}(b c)
$$

Para demonstrar o Lema 6.3.1, será preciso um outro lema.

Lema 6.3.2. Sejam $a \geq 2$ e $b, c, x \geq 1$ inteiros. Vale que

$$
\left(a \uparrow^{x} b\right)\left(a \uparrow^{x} c\right) \leq a \uparrow^{x}(b+c)
$$

Demonstração do Lema 6.3.2. Faz-se uma indução em $x$. Quando $x=1$, a operação de uma flecha é a exponenciação. Para quaisquer $a \geq 2$ e $b, c \geq 1$, vale que $a^{b} \cdot a^{c}=a^{b+c}$.

Seja $x \geq 1$ e suponha que para quaisquer $a \geq 2$ e $b, c \geq 1$, vale que $\left(a \uparrow^{x} b\right)\left(a \uparrow^{x} c\right) \leq a \uparrow^{x}(b+c)$. Para provar que o mesmo vale para $x+1$, será feita uma indução em $b$. Quando $b=1$,

$$
\left(a \uparrow^{x+1} 1\right)\left(a \uparrow^{x+1} c\right)=a \cdot\left(a \uparrow^{x+1} c\right) \leq a \uparrow^{x}\left(a \uparrow^{x+1} c\right)=a \uparrow^{x+1}(c+1)
$$

Seja $b \geq 1$ e suponha que $\left(a \uparrow^{x+1} b\right)\left(a \uparrow^{x+1} c\right) \leq a \uparrow^{x+1}(b+c)$ para qualquer $c \geq 1$. Para $b+1$, faça primeiro o caso em que $c=1$. Tem-se

$$
\left(a \uparrow^{x+1}(b+1)\right)\left(a \uparrow^{x+1} 1\right)=a \cdot\left(a \uparrow^{x+1}(b+1)\right) \leq a \uparrow^{x}\left(a \uparrow^{x+1}(b+1)\right)=a \uparrow^{x+1}(b+2) .
$$

Para o caso $c \geq 2$, tem-se

$$
\left(a \uparrow^{x+1}(b+1)\right)\left(a \uparrow^{x+1} c\right)=\left(a \uparrow^{x}\left(a \uparrow^{x+1} b\right)\right)\left(a \uparrow^{x}\left(a \uparrow^{x+1}(c-1)\right)\right)
$$

Utilizando a hipótese da indução em $x$, tem-se que

$$
\begin{aligned}
\left(a \uparrow^{x+1}(b+1)\right)\left(a \uparrow^{x+1} c\right) & \leq a \uparrow^{x}\left(\left(a \uparrow^{x+1} b\right)+\left(a \uparrow^{x+1}(c-1)\right)\right) \\
& \leq a \uparrow^{x}\left(\left(a \uparrow^{x+1} b\right) \cdot\left(a \uparrow^{x+1}(c-1)\right)\right),
\end{aligned}
$$

onde a segunda desigualdade vale, pois $\left(a \uparrow^{x+1} b\right),\left(a \uparrow^{x+1} c\right) \geq a \geq 2$. Utilizando a hipótese da 
indução em $b$, tem-se

$$
\left(a \uparrow^{x+1}(b+1)\right)\left(a \uparrow^{x+1} c\right) \leq a \uparrow^{x}\left(a \uparrow^{x+1}(b+c-1)\right)=a \uparrow^{x+1}(b+c)
$$

Pode-se demonstrar o Lema 6.3.1.

Demonstração do Lema 6.3.1. É feita uma indução em $x$. Para $x=0$, a operação de 0 flechas é a multiplicação. Portanto, $\left(a \uparrow^{0} b\right) \uparrow^{0} c=a b c=a \uparrow^{0}(b c)$. Seja $x \geq 0$ e, para quaisquer $a \geq 2$ e $b, c \geq 1$, suponha que $\left(a \uparrow^{x} b\right) \uparrow^{x} c \leq a \uparrow^{x}(b c)$.

Para provar que a propriedade vale para $x+1$, é feita uma indução em $c$. Quando $c=1$, temse $\left(a \uparrow^{x+1} b\right) \uparrow^{x+1} 1=a \uparrow^{x+1} b=a \uparrow^{x+1}(b \cdot 1)$. Seja $c \geq 1$ e suponha que, para quaisquer $a \geq 2$ e $b \geq 1$, tem-se $\left(a \uparrow^{x+1} b\right) \uparrow^{x+1} c \leq a \uparrow^{x+1}(b c)$.

Para $c+1$, o caso em que $b=1$ é imediato, tem-se $\left(a \uparrow^{x+1} 1\right) \uparrow^{x+1}(c+1)=a \uparrow^{x+1}(1(c+1))$. Considere $b \geq 2$, tem-se

$$
\left(a \uparrow^{x+1} b\right) \uparrow^{x+1}(c+1)=\left(a \uparrow^{x+1} b\right) \uparrow^{x}\left(\left(a \uparrow^{x+1} b\right) \uparrow^{x+1} c\right) .
$$

Utilizando a hipótese da indução em $c$, tem-se

$$
\begin{aligned}
\left(a \uparrow^{x+1} b\right) \uparrow^{x+1}(c+1) & \leq\left(a \uparrow^{x+1} b\right) \uparrow^{x}\left(a \uparrow^{x+1}(b c)\right) \\
& =\left(a \uparrow^{x}\left(a \uparrow^{x+1}(b-1)\right)\right) \uparrow^{x}\left(a \uparrow^{x+1}(b c)\right)
\end{aligned}
$$

Utilizando a hipótese da indução em $x$, tem-se

$$
\left(a \uparrow^{x+1} b\right) \uparrow^{x+1}(c+1) \leq a \uparrow^{x}\left(\left(a \uparrow^{x+1}(b-1)\right) \cdot\left(a \uparrow^{x+1}(b c)\right)\right) .
$$

Utilizando o Lema 6.3.2, tem-se

$$
\begin{aligned}
\left(a \uparrow^{x+1} b\right) \uparrow^{x+1}(c+1) & \leq a \uparrow^{x}\left(a \uparrow^{x+1}(b-1+b c)\right) \\
& =a \uparrow^{x+1}(b+b c)=a \uparrow^{x+1}(b(c+1)) .
\end{aligned}
$$

Uma última observação antes de provar uma cota superior é sobre a seguinte propriedade das flechas.

Lema 6.3.3. Sejam $k \geq 2, a \geq 2, b \geq 1$ e $x \geq 1$ inteiros. Vale que $k\left(a \uparrow^{x} b\right) \leq(k a) \uparrow^{x} b$.

Demonstração. A prova dada é por indução em $b$. Para $b=1$, tem-se $k\left(a \uparrow^{x} 1\right)=k a=(k a) \uparrow^{x} 1$.

Seja $b \geq 1$ e suponha que $k\left(a \uparrow^{x} b\right) \leq(k a) \uparrow^{x} b$ para quaisquer $k \geq 2, a \geq 2$ e $x \geq 1$.

Prova-se a afirmação para $b+1$. Utilizando o Lema 5.3.4, tem-se

$$
\begin{aligned}
k\left(a \uparrow^{x}(b+1)\right) & =k\left(a \uparrow^{x-1}\left(a \uparrow^{x} b\right)\right) \\
& \leq a \uparrow^{x-1}\left(\left(a \uparrow^{x} b\right)+k\right) \\
& \leq a \uparrow^{x-1}\left(k \cdot\left(a \uparrow^{x} b\right)\right),
\end{aligned}
$$


onde a última desigualdade vale, pois $y+z \leq y z$ quando $y, z \geq 2$. Pela hipótese da indução em $b$, tem-se

$$
\begin{aligned}
k\left(a \uparrow^{x}(b+1)\right) & \leq a \uparrow^{x-1}\left((k a) \uparrow^{x} b\right) \\
& \leq(k a) \uparrow^{x-1}\left((k a) \uparrow^{x} b\right) \\
& =(k a) \uparrow^{x}(b+1) .
\end{aligned}
$$

Prova-se a seguinte cota superior.

Teorema 6.3.4. Sejam $d \geq 2, r \geq 1$ e $g \geq 4$ inteiros. Vale que $m(d, r, g) \leq d \uparrow^{g-2} 6^{r}$.

Demonstração. Como foi dito, a prova é por indução em $g$. Para $g=4$, tem-se

$$
m(d, r, 4)=2 r+1 \leq 2 \uparrow^{0}(r+1) \leq d \uparrow^{g-2} 6^{r}
$$

Seja $g \geq 4$ e suponha que $m(d, r, g) \leq d \uparrow^{g-2} 6^{r}$ para todo $d \geq 2$ e $r \geq 1$.

Para provar que a cota vale para $g+1$, será feita uma indução em $r$. Para $r=1$, utiliza-se a relação 2 do Teorema 6.1 .1 e a hipótese da indução em $g$. Tem-se

$$
\begin{aligned}
m(d, 1, g+1) & \leq m(d, d, g)+1 \\
& \leq\left(d \uparrow^{g-2} 6^{d}\right)+1 \leq 2\left(d \uparrow^{g-2} 6^{d}\right) \leq d \uparrow^{g-2}\left(6^{d}+2\right),
\end{aligned}
$$

na última desigualdade foi usado o Lema 5.3.4. Prova-se agora que $6^{d}+2 \leq d \uparrow^{g-1} 3$. De fato,

$$
\begin{aligned}
6^{d}+2 & \leq(6+2)^{d} \leq\left(2^{2^{2}}\right)^{d} \leq d^{d \cdot\left(d^{d}\right)}=d^{d^{d+1}} \leq d^{d^{d^{d}}}=d \uparrow^{2} 4 \\
& =d \uparrow^{2}\left(2^{2}\right) \leq d \uparrow^{2}(d \uparrow d) \leq d \uparrow^{2}\left(d \uparrow^{2} d\right)=d \uparrow^{3} 3 \leq d \uparrow^{g-1} 3 .
\end{aligned}
$$

Portanto,

$$
m(d, 1, g+1) \leq d \uparrow^{g-2}\left(d \uparrow^{g-1} 3\right)=d \uparrow^{g-1} 4
$$

Além disso, $m(d, 1, g+1) \leq d \uparrow^{g-1} 4 \leq d \uparrow^{g-1} 6$.

Seja $r \geq 1$ e suponha que $m(d, r, g+1) \leq d \uparrow^{g-1} 6^{r}$ para qualquer $d \geq 2$. Prova-se que a cota vale para $m(d, r+1, g+1)$. Utilize a relação 3 do Teorema 6.1.1, a relação (6.3) obtida para o caso $r=1$ e a hipótese da indução em $r$ para escrever

$$
\begin{aligned}
m(d, r+1, g+1) & \leq m(d, 1, g+1)+m\left(d^{m(d, 1, g+1)}, r, g+1\right) \\
& \leq d \uparrow^{g-1} 4+\left(d \uparrow\left(d \uparrow^{g-1} 4\right)\right) \uparrow^{g-1} 6^{r} \\
& \leq 2\left(\left(d \uparrow^{g-1} 5\right) \uparrow^{g-1} 6^{r}\right) \\
& \leq\left(2\left(d \uparrow^{g-1} 5\right)\right) \uparrow^{g-1} 6^{r} \\
& \leq\left(d \uparrow^{0}\left(d \uparrow^{g-1} 5\right)\right) \uparrow^{g-1} 6^{r} \\
& \leq\left(d \uparrow^{g-1} 6\right) \uparrow^{g-1} 6^{r}
\end{aligned}
$$


em (6.4), foi utilizado o Lema 6.3.3. Por fim, utilizando o Lema 6.3.1, tem-se

$$
m(d, r+1, g+1) \leq d \uparrow^{g-1}\left(6^{r+1}\right)
$$

A partir dessa cota superior para $m(d, r, g)$, tem-se o seguinte resultado para o número de vértices do grafo com cintura e número cromático grandes $\mathcal{T}_{2, k, g}$.

Corolário 6.3.5. Para $k \geq 3$ e $g \geq 2$, vale que $\left|\mathcal{T}_{2, k, g}\right| \leq(k-1) \uparrow^{2 g-1}\left(6^{k}+1\right)$.

Demonstração. Para construir $\mathcal{T}_{2, k, g}$, utiliza-se um $(k-1, k, 2 g+1)$-grafo $G$. Se $h$ é a altura da árvore de $G$, como visto em (6.1), tem-se

$$
\left|V\left(\mathcal{T}_{2, k, g}\right)\right|=\frac{(k-1)^{h}-1}{k-2}<(k-1)^{h} .
$$

Por definição, $h \leq m(k-1, k, 2 g+1)$. Pelo Teorema 6.3.4, tem-se $h \leq(k-1) \uparrow^{2 g-1} 6^{k}$. Assim,

$$
\left|V\left(\mathcal{T}_{2, k, g}\right)\right|<(k-1) \uparrow\left((k-1) \uparrow^{2 g-1} 6^{k}\right) \leq(k-1) \uparrow^{2 g-1}\left(6^{k}+1\right) .
$$

\subsubsection{Uma cota inferior para $m(d, r, g)$}

A altura mínima da árvore $T$ de um $(d, r, g)$-grafo $G$ precisa ser grande; será visto que o valor de $m(2, r, g)$ é Ackermanniano. Primeiro, são feitas algumas considerações simples sobre a altura de $T$.

Considere uma folha $v$ de $T$. Como $r \geq 1$, pode-se tomar o ancestral $x$ mais próximo de $v$ atingido por uma aresta de retorno. A distância entre $x$ e $v$ na árvore precisa ser pelo menos $g-1$ para não formar circuito de comprimento menor que $g$. Suponha que cada folha envie mais arestas de retorno; considere que $r \geq 2$. Sejam $x_{1}$ e $x_{2}$ dois dos ancestrais de $v$ atingidos por arestas de retorno de $G$. Se a distância entre $x_{1}$ e $x_{2}$ em $T$ for menor que $g-2$, adicionando-se $v$ e as arestas de retorno $v x_{1}$ e $v x_{2}$ ao caminho mínimo em $T$ entre $x_{1}$ e $x_{2}$, obtém-se um circuito de comprimento menor que $g$. Como todos ancestrais de $v$ atingidos por arestas de retorno estão no caminho completo até $v$, tem-se que esse caminho tem comprimento pelo menos $r(g-2)$. Portanto,

$$
m(d, r, g) \geq r(g-2) .
$$

Essa cota inferior considera apenas uma folha. Utilizando-se o fato de que mais folhas enviam cada uma $r$ arestas de retorno, pode-se aumentar a cota inferior.

Antes de se considerar mais folhas, tem-se a seguinte definição para árvores completas.

Definição 6.3.1. Seja $T$ uma árvore completa. Para qualquer vértice $v$ de $T$, o nível de $v$, denotado por $n(v)$ ou $n_{T}(v)$, é a distância de $v$ até a folha mais próxima.

Se $v$ é folha, tem-se que $n(v)=0$. Se $v$ é vizinho de uma folha, tem-se que $n(v)=1$. O nível da raiz é igual à altura da árvore. 
Suponha que $d \geq 2, r \geq 1$ e $g \geq 5$. Acompanhe com a Figura 6.9. Pelo que foi dito, sabe-se que $T$ tem altura pelo menos $g-1$. Considere um vértice $x$ que esteja no nível $n(x)=\lceil g / 2\rceil-2<$ $g-1$. Sejam $u$ e $v$ duas folhas de $T$ que estejam na subárvore com raiz em $x$. Assim, o menor caminho $(u, \ldots, v)$ em $T$ entre $u$ e $v$ tem comprimento no máximo $2(\lceil g / 2\rceil-2) \leq 2(g / 2+1 / 2)-4=$ $g-3$. Observe as arestas de retorno de $u$ e $v$. A aresta de retorno mais baixa de cada uma dessas folhas está pelo menos no nível $g-1$, que é acima de $n(x)$. Assim, tanto as arestas de retorno que partem de $u$ quanto as que partem de $v$ atingem vértices do caminho em $T$ entre $x$ e a raiz. Se uma aresta de retorno de $u$ atingir um certo ancestral $y$ que também seja atingido por uma aresta de retorno de $v$, tem-se que $(u, \ldots, v, y)$ é um circuito de comprimento no máximo $g-1$. Dessa forma, os ancestrais atingidos por $u$ precisam ser distintos dos atingidos por $v$. Existem $d^{n(x)}$ folhas na subárvore com raiz $x$. Assim, no caminho em $T$ a partir de $x$ até a raiz, atinge-se o nível $g-1$ e, depois, ainda se tem mais pelo menos $r d^{n(x)}-1$ vértices. Dessa forma,

$$
m(d, r, g) \geq g+r d^{\lceil g / 2\rceil-2}-2 .
$$

Consegue-se não apenas uma cota inferior linear em $g$, mas sim exponencial em $g$.

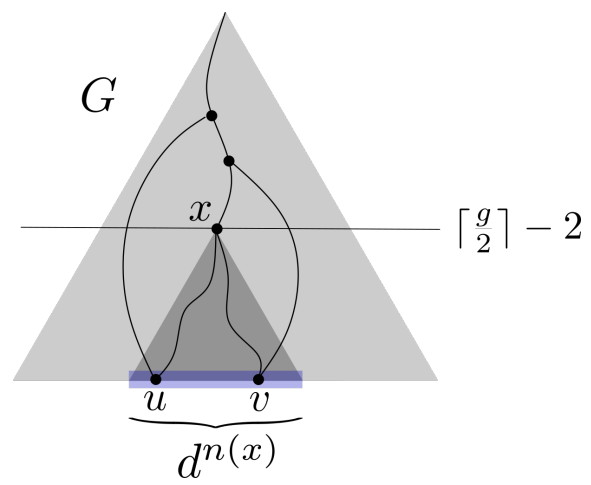

Figura 6.9: As folhas da subárvore atingem ancestrais distintos no caminho entre $x$ e a raiz.

O que será provado é uma cota inferior cujo número de flechas de Knuth depende de $g$. Isso será feito de forma parecida. Será escolhido um certo subconjunto das folhas de $T$, que tenha certa propriedade com relação aos seus ancestrais atingidos por arestas de retorno e que seja grande o bastante. A restrição sobre a cintura determinará que alguma aresta de retorno com extremo nesse conjunto atinge um nível muito alto.

Os fatores relevantes para garantir que um nível alto seja atingido por alguma aresta de retorno são apenas um certo subconjunto das folhas, as arestas de retorno que incidem nessas folhas e a restrição sobre a cintura. Assim, em vez dos $(d, r, g)$-grafos, será considerada uma família de grafos mais simples, cujos vértices também são os mesmos de uma árvore, mas que mantém apenas os fatores que são relevantes para que a altura mínima da árvore seja grande. Será visto que, a partir de um $(2, r, g)$-grafo $G$, sempre se poderá obter um grafo dessa família cuja árvore tenha quase a mesma altura que a árvore de $G$. Dessa forma, a altura mínima da árvore de um grafo dessa família será basicamente menor que ou igual a $m(2, r, g)$. Essa nova família de grafos são os $T B(x, r, g)$ grafos.

Definição 6.3.2. Sejam $x, r$ e $g$ inteiros positivos. Um grafo $G$ é um $T B(x, r, g)$-grafo quando existem uma árvore binária completa $T_{G}$, um inteiro $x^{\prime} \geq x$ e um subconjunto das folhas $L_{G} \subset F\left(T_{G}\right)$ com $\left|L_{G}\right| \geq 2^{-x^{\prime} / 4}\left|F\left(T_{G}\right)\right|$ tais que valem as seguintes propriedades, representadas na Figura 6.10. 
1. $V(G)=V\left(T_{G}\right)$.

2. $E(G)=\left\{u v_{1}, \ldots, u v_{r}: u \in L_{G}, v_{1} \neq \cdots \neq v_{r}\right.$ ancestrais de $u$ e $\left.n\left(v_{1}\right), \ldots, n\left(v_{r}\right)>x^{\prime}\right\}$.

3. $\operatorname{cin}(G)>g$.

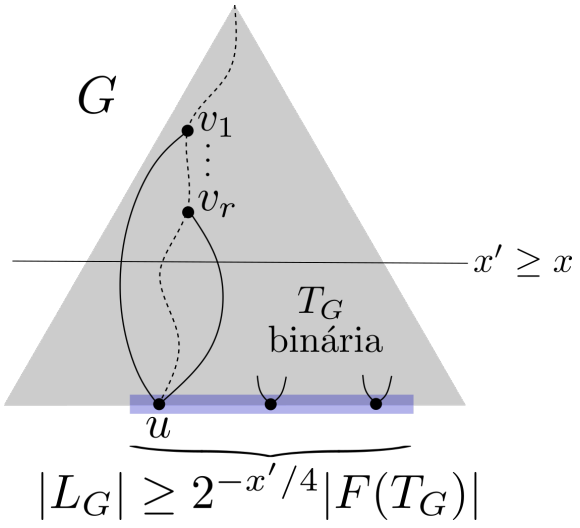

Figura 6.10: $O$ grafo $G$ é um $T B(x, r, g)$-grafo.

A família $T B(x, r, g)$ é formada por grafos $G$ cujas únicas arestas são $r$ arestas de retorno para cada folha de um subconjunto grande das folhas $L_{G}$. O conjunto $L_{G}$ poderá ser denotado por $L$, quando for claro a qual $T B(x, r, g)$ ele se refere. As arestas de retorno de $G$ ligam as folhas de $L_{G}$ a ancestrais que estejam a pelo menos uma distância $x^{\prime} \geq x$ delas. Tudo isso, sem formar circuitos de comprimento menor que ou igual a $g$.

Note que, diferentemente do que ocorre em um $(2, r, g)$-grafo, as arestas da árvore não estão incluídas e não é necessário que todas as folhas tenham $r$ arestas de retorno. Desse modo, se $g \geq x+1$, um $(2, r, g)$-grafo contém um $T B(x, r, g-1)$-grafo, basta remover as arestas da árvore. Assim, a altura mínima da árvore de um $T B(x, r, g-1)$-grafo é menor que ou igual à altura mínima da árvore de um $(2, r, g)$-grafo, com $g \geq x+1$.

Para determinar a altura mínima da árvore de um $T B(x, r, g)$-grafo, será utilizado o seguinte parâmetro.

Definição 6.3.3. Sejam $x \geq 100, r \geq 2$ e $g \geq 4$ inteiros. O parâmetro $\ell(x, r, g)$ está associado à família $T B(x, r, g)$. Considere o conjunto $\mathbb{L}(x, r, g)$ de todos os inteiros $\ell$ que satisfazem a seguinte propriedade. Dado $G \in T B(x, r, g)$ com árvore $T_{G}$, existem $\ell^{\prime} \geq \ell$ e $A_{G, \ell^{\prime}} \subset F\left(T_{G}\right)$ tais que

1. $\left|A_{G, \ell^{\prime}}\right| \geq 2^{-\ell^{\prime} / 8}\left|F\left(T_{G}\right)\right|$,

2. se $v \in A_{G, \ell^{\prime}}$, então existe $u \in V(G)$ tal que $v u \in E(G)$ e $n(u)>\ell^{\prime}$.

A Figura 6.11 representa essas propriedades. Define-se $\ell(x, r, g)$ como o máximo de $\mathbb{L}(x, r, g)$.

Dado $G \in T B(x, r, g)$, um valor $\ell^{\prime}$ para o qual pode ser encontrado um $A_{G, \ell^{\prime}} \subset F\left(T_{G}\right)$ satisfazendo as propriedades 1 e 2 da Definição 6.3.3 será dito válido para $G$. Um valor $\ell$ que seja menor que ou igual a pelo menos um valor válido para cada grafo de $T B(x, r, g)$ pertence a $\mathbb{L}(x, r, g)$.

As propriedades impostas sobre $A_{G, \ell^{\prime}}$ significam que, garantindo que pelo menos uma aresta de retorno de cada folha de $A_{G, \ell^{\prime}}$ atinja um nível alto, a fração das folhas que devem pertencer a $A_{G, \ell^{\prime}}$ não precisa ser tão grande. Isso porque, garantindo que a árvore possui níveis altos, também 


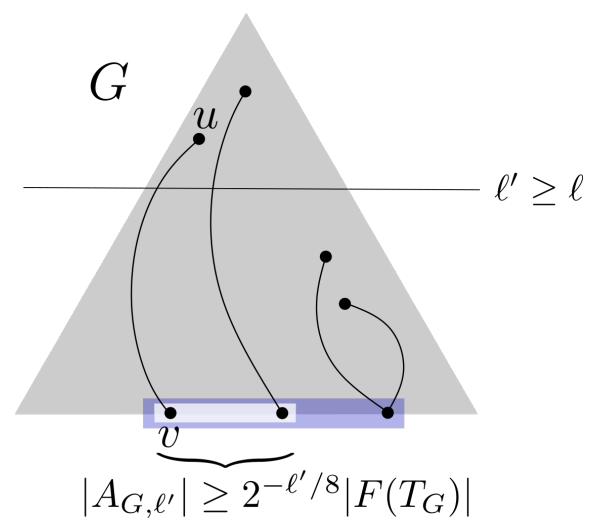

Figura 6.11: Tome $\ell \in \mathbb{L}(x, r, g)$. Para cada $G \in T B(x, r, g)$, existem $\ell^{\prime} \geq \ell$ e um conjunto $A_{G, \ell^{\prime}} \subset F\left(T_{G}\right)$

vale que ela tem muitas folhas. Desse modo, uma fração menor das folhas ainda será um conjunto grande.

Assim, dado $G \in T B(x, r, g)$, para existir $\ell^{\prime}$ válido, um conjunto grande das folhas deve enviar pelo menos uma aresta para algum nível alto. Não é claro sempre haverá um $\ell^{\prime}$ válido. Mas será provado no Lema 6.3.7 que, para certos valores de $x, r$ e $g$, todo grafo de $T B(x, r, g)$ terá um valor válido, de modo que o conjunto $\mathbb{L}(x, r, g)$ não será vazio. Antes, discute-se em quais casos não se tem um valor válido para todo grafo de $T B(x, r, g)$.

Para a família $T B(x, r, g)$, um valor $\ell \in \mathbb{L}(x, r, g)$ e o termo $x$ satisfazem condições semelhantes. Dado $G \in T B(x, r, g)$, existem $x^{\prime} \geq x$ e $\ell^{\prime} \geq \ell$ e subconjuntos das folhas $L$ e $A_{G, \ell^{\prime}}$ tais que, em $L$, cada folha envia $r$ arestas de retorno para níveis acima de $x^{\prime}$ e, em $A_{G, \ell^{\prime}}$, cada folha envia pelo menos uma aresta de retorno para um nível acima de $\ell^{\prime}$. Além disso, os tamanhos de $L$ e $A_{G, \ell^{\prime}}$ são pelo menos uma fração $2^{-x^{\prime} / 4}$ e $2^{-\ell^{\prime} / 8}$ das folhas, respectivamente. Assim, é natural perguntar-se como $x$ e $\ell(x, r, g)$ se relacionam.

Uma observação é a seguinte. Seja $G \in T B(x, r, g)$ e suponha que existe $\ell^{\prime}$ válido para $G$. Como apenas as folhas em $L$ enviam arestas de retorno, tem-se $A_{G, \ell^{\prime}} \subset L$. Além disso, o tamanho de $A_{G, \ell^{\prime}}$ é pelo menos uma fração $2^{-\ell^{\prime} / 8}$ das folhas, enquanto o de $L$, uma fração $2^{-x^{\prime} / 4} \leq 2^{-x / 4}$. Dessa forma, é preciso que $2 x \leq \ell^{\prime}$, para todo $G \in T B(x, r, g)$. Assim, $2 x \in \mathbb{L}(x, r, g)$. Isso significa que $2 x \leq \ell(x, r, g)$ e que todo grafo em $T B(x, r, g)$ tem alguma folha que envia uma aresta de retorno para um nível maior que $2 x$.

Feita essa observação, afirma-se que não pode haver valor $\ell^{\prime}$ válido para todo grafo de $T B(x, 1, g)$, com $x \geq 1$ e $g \geq 1$. Basta encontrar um grafo $H \in T B(x, 1, g)$ com árvore $T_{H}$ tal que nenhuma de suas folhas envia uma aresta de retorno para um nível acima de $2 x$. Construa $H$ a partir dos vértices de uma árvore binária completa $T_{H}$. Para cada folha $v \in F\left(T_{H}\right)$, o grafo $H$ terá uma aresta de retorno $v u, \operatorname{com} n(u)=x+1$ (Figura 6.12). Tem-se que $H$ é uma união disjunta de estrelas; as folhas são os vértices de grau 1 e os ancestrais dessas folhas no nível $x+1$ são os vértices centrais. Dessa forma, a cintura de $H$ é infinita. Conclui-se que $H \in T B(x, 1, g)$, com $x^{\prime}=x$ e $L=F\left(T_{H}\right)$. Como $x+1 \leq 2 x$, nenhuma folha de $H$ envia aresta para um nível acima de $2 x$. Assim, o conjunto $\mathbb{L}(x, 1, g)$ é vazio e não se define $\ell(x, 1, g)$.

Quando $r=1$, um grafo $G \in T B(x, 1, g)$ é sempre uma união disjunta de estrelas, portanto tem cintura infinita. Dessa forma, a restrição $\operatorname{cin}(G)>g$ é sempre satisfeita automaticamente, sem forçar que as arestas de retorno atinjam níveis altos. O mesmo ocorre para quaisquer $r \geq 1$ e $x \geq 1$, 


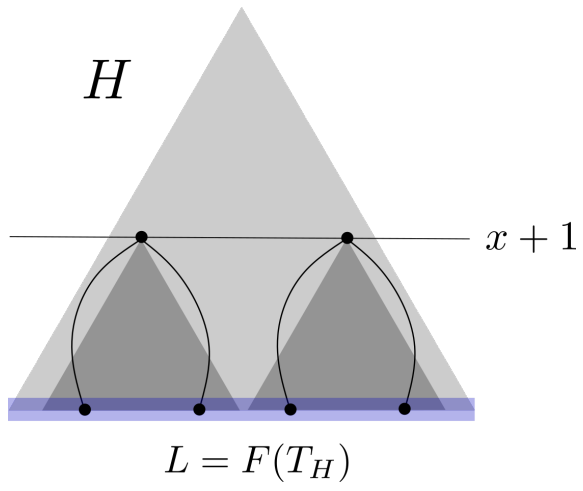

Figura 6.12: Para este TB $(x, 1, g)$-grafo $H$ não há como tomar $\ell^{\prime}$ válido.

quando $1 \leq g \leq 3$, pois todo $T B(x, r, g)$-grafo é bipartido e, portanto, tem cintura pelo menos 4 . Assim, quando $1 \leq g \leq 3$, as arestas de retorno também não são forçadas a atingir níveis altos.

Afirma-se que, para $1 \leq g \leq 3$, se $r$ não for grande o bastante, existirá um grafo em $T B(x, r, g)$ para o qual não se tem um valor $\ell^{\prime}$ válido. Construa um grafo $H$ a partir dos vértices de uma árvore binária completa $T_{H}$. Para todo $v \in F\left(T_{H}\right)$, adicione $r$ arestas de retorno que ligam $v$ a seus ancestrais nos níveis $x+1$ a $x+r$ (Figura 6.13). O menor circuito de $H$ tem comprimento 4 , portanto $H \in T B(x, r, g)$. O nível mais alto atingido por uma aresta de retorno de $H$ é $x+r$. Pelo que foi observado anteriormente, um valor $\ell^{\prime}$ válido para $H$ satisfaz que $2 x \leq \ell^{\prime} \leq x+r-1$. Dessa forma, quando $1 \leq g \leq 3$ e $r<x+1$, não existe $\ell^{\prime}$ válido para $H$; tem-se que $\mathbb{L}(x, r, g)$ é vazio e não se define $\ell(x, r, g)$.

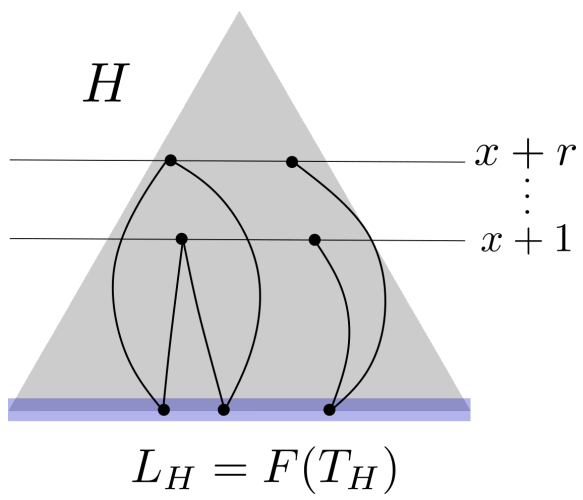

Figura 6.13: Quando $3 \geq g \geq 1$ e $r<x+1$, não se define $\ell(x, r, g)$.

Será visto que, tomando $r \geq 2, g \geq 4$ e $x \geq 100$, todo grafo $G \in T B(x, r, g)$ terá um valor $\ell^{\prime}$ válido. Assim, o conjunto $\mathbb{L}(x, r, g)$ não será vazio. O valor máximo $\ell(x, r, g)$ estará bem definido, pois todo valor em $\mathbb{L}(x, r, g)$ é menor que a altura mínima da árvore de um grafo em $T B(x, r, g)$. De fato, por definição, dado qualquer $\ell \in \mathbb{L}(x, r, g)$ e $G \in T B(x, r, g)$, a árvore $T_{G}$ deve ter um subconjunto não vazio de folhas que enviam arestas para níveis acima de $\ell^{\prime} \geq \ell$, de modo que a altura de $G$ é maior que $\ell$.

Primeiro, tem-se a seguinte proposição.

Proposição 6.3.6. Sejam $x, r, g$ inteiros positivos. Vale que $\mathbb{L}(x, r, g)$ está contido em $\mathbb{L}(x+1, r, g)$, em $\mathbb{L}(x, r+1, g)$ e em $\mathbb{L}(x, r, g+1)$. Assim, o parâmetro $\ell(x, r, g)$ é crescente em qualquer uma das três variáveis.

Demonstração. Primeiro prova-se que $\mathbb{L}(x, r, g) \subset \mathbb{L}(x, r+1, g)$. Afirma-se que, dado $G \in T B(x, r+$ 
$1, g)$ com árvore $T_{G}$, existe $G^{\prime} \in T B(x, r, g)$ cuja árvore também é $T_{G}$ e $G^{\prime} \subset G$. Para ver isso, tome $G$ e remova uma aresta de retorno de cada folha em $L_{G}$; o grafo resultante é $G^{\prime}$. De fato, $\operatorname{cin}\left(G^{\prime}\right) \geq$ $\operatorname{cin}(G)>g$ e os mesmos $x^{\prime}$ e $L_{G}=L_{G^{\prime}}$ garantem que $G^{\prime} \in T B(x, r, g)$.

Seja $\ell \in \mathbb{L}(x, r, g)$ e $G \in T B(x, r+1, g)$. Se for encontrado $\ell^{\prime} \geq \ell$ que seja válido para $G$, tem-se que $\ell \in \mathbb{L}(x, r+1, g)$. Tome $G^{\prime} \subset G$ como foi descrito. Como $G^{\prime} \in T B(x, r, g)$ e $T_{G^{\prime}}=T_{G}$, existe $\ell^{\prime \prime} \geq \ell$ e $A_{G^{\prime}, \ell^{\prime \prime}} \subset F\left(T_{G}\right)$ com $\left|A_{G^{\prime}, \ell^{\prime \prime}}\right| \geq 2^{-\ell^{\prime \prime} / 8}\left|F\left(T_{G}\right)\right|$, em que cada folha envia pelo menos uma aresta para um nível acima de $\ell^{\prime \prime}$. O valor $\ell^{\prime}=\ell^{\prime \prime} \geq \ell$ é válido para $G$, pois pode-se tomar $A_{G, \ell^{\prime}}=A_{G^{\prime}, \ell^{\prime \prime}}$.

Em seguida, prova-se que $\mathbb{L}(x, r, g) \subset \mathbb{L}(x, r, g+1)$. Seja $\ell \in \mathbb{L}(x, r, g)$ e $G \in T B(x, r, g+1)$. Mais uma vez, quer-se $\ell^{\prime} \geq \ell$ que seja válido para $G$. Basta notar que todo $T B(x, r, g+1)$-grafo é também um $T B(x, r, g)$-grafo. De fato, se um grafo é tal que todo circuito tem comprimento maior que $g+1$, então sua cintura é também maior que $g$. Assim, $G \in T B(x, r, g+1) \subset T B(x, r, g)$. Como $\ell \in \mathbb{L}(x, r, g)$, existe $\ell^{\prime} \geq \ell$ válido para $G$.

Para ver que $\mathbb{L}(x, r, g) \subset \mathbb{L}(x+1, r, g)$, note que $T B(x+1, r, g) \subset T B(x, r, g)$-grafo. De fato, vale que $x^{\prime} \geq x+1 \geq x$ e, portanto, para dado $G \in T B(x+1, r, g)$, os mesmos $x^{\prime}$ e $L_{G}$ garantem que $G \in T B(x, r, g)$.

Para provar que $\mathbb{L}(x, r, g)$ não é vazio para $x \geq 100, r \geq 2$ e $g \geq 4$, pela Proposição 6.3.6, basta provar que $\mathbb{L}(100,2,4)$ não é vazio. Será utilizada a seguinte definição.

Definição 6.3.4. Dada uma função $f(x)$, defina

$$
\begin{aligned}
& f_{0}(x)=x \mathrm{e} \\
& f_{i}(x)=f\left(f_{i-1}(x)\right), \text { para } i \geq 1 .
\end{aligned}
$$

Encontrar $\ell \in \mathbb{L}(x, r, g)$ mostra que o conjunto não é vazio e também fornece uma cota inferior para $\ell(x, r, g)$. Assim, tem-se o seguinte lema.

Lema 6.3.7. Para $x \geq 100$ e $r \geq 2$, tem-se

$$
\ell(x, r, 4) \geq f_{r-1}(x) \text {, onde } f(y)=y+\left\lfloor 2^{y / 4}\right\rfloor .
$$

Demonstração. Para $x \geq 100$ e $r \geq 2$, procura-se um valor $\ell \in \mathbb{L}(x, r, 4)$. Isto é, dado $G \in$ $T B(x, r, 4)$ com árvore $T_{G}$, é preciso existir $\ell^{\prime} \geq \ell$ e $A_{G, \ell^{\prime}} \in F\left(T_{G}\right)$ com $\left|A_{G, \ell^{\prime}}\right| \geq 2^{-\ell^{\prime} / 8}\left|F\left(T_{G}\right)\right|$ e tal que cada folha em $A_{G, \ell^{\prime}}$ envie pelo menos uma aresta de retorno para algum nível maior que $\ell^{\prime}$.

Como $G \in T B(x, r, 4)$, tem-se $x^{\prime} \geq x$ e $L_{G}$ conforme a definição de $T B(x, r, 4)$. Será encontrado um $\ell^{\prime}$ válido para $G$. Acompanhe a ideia que se segue com a Figura 6.14. A ideia é dividir os níveis acima de $x^{\prime}$ em intervalos e provar que um número grande de folhas envia no máximo uma aresta de retorno para níveis dentro de um mesmo intervalo; essas folhas serão $A_{G, \ell^{\prime}}$. Como as folhas em $L_{G}$ enviam pelo menos $r$ arestas de retorno, então $r$ intervalos distintos serão atingidos por boa parte das folhas. Enumerando os intervalos a partir daquele que contém os níveis mais baixos, o extremo inferior do $r$-ésimo intervalo será $\ell^{\prime}+1$.

Assim, particione os níveis acima de $x^{\prime}$ em conjuntos $I_{i}(i \geq 0)$, onde

$$
I_{i}=\left\{n \in \mathbb{N}: n \in\left[f_{i}\left(x^{\prime}\right)+1, f_{i+1}\left(x^{\prime}\right)\right]\right\} .
$$




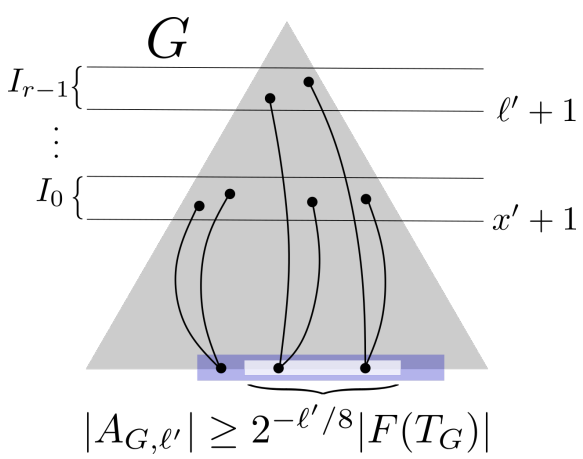

Figura 6.14: Para encontrar $\ell^{\prime}$ válido, particione os niveis e mostre que um conjunto grande das folhas não repete intervalo.

O primeiro intervalo $I_{0}$ compreende os níveis em $\left[x^{\prime}+1, x^{\prime}+\left\lfloor 2^{x^{\prime} / 4}\right\rfloor\right]$ e assim por diante. A notação $I_{i}$ também será utilizada para se referir aos vértices da árvore em questão que tenham níveis em $I_{i}$.

Como dito, o interesse está nas folhas que enviam no máximo uma aresta de retorno para cada intervalo, pois são elas serão $A_{G, \ell^{\prime}}$. O tamanho desse conjunto de interesse será analisado considerando-se seu complemento. Assim, para cada $i \geq 0$, tome o conjunto das folhas que enviam mais de uma aresta para $I_{i}$,

$$
A_{i}=\left\{v \in F\left(T_{G}\right): \exists v u_{1} \neq v u_{2} \in E(G) \operatorname{com} n\left(u_{1}\right), n\left(u_{2}\right) \in I_{i}\right\}
$$

O que se quer mostrar é que $\left|\bigcup_{i \geq 0} A_{i}\right|$ não pode ser uma fração muito grande de $L_{G}$. Dessa forma, sobram muitos vértices em $L_{G}$ tais que quaisquer duas de suas $r$ arestas de retorno sempre atinjam intervalos distintos.

Para limitar $\left|\bigcup_{i \geq 0} A_{i}\right|$, prova-se para cada $A_{i}$, que

$$
\left|A_{i}\right|<2^{-f_{i}\left(x^{\prime}\right) / 2}\left|F\left(T_{G}\right)\right|
$$

Suponha por contradição que existe $i_{0} \geq 0$ tal que $\left|A_{i_{0}}\right| \geq 2^{-f_{i_{0}}\left(x^{\prime}\right) / 2}\left|F\left(T_{G}\right)\right|$. A Figura 6.15 ilustra o argumento que segue. Considere as subárvores com raizes no nível $f_{i_{0}}\left(x^{\prime}\right)+1$, que é o nível mais baixo em $I_{i_{0}}$. Toda folha de $T_{G}$ é folha de alguma dessas subárvores. Dessa forma, existe alguma subárvore $T_{G}^{\prime}$ tal que $\left|F\left(T_{G}^{\prime}\right) \cap A_{i_{0}}\right| \geq 2^{-f_{i_{0}}\left(x^{\prime}\right) / 2}\left|F\left(T_{G}^{\prime}\right)\right| ;$ uma fração grande das folhas de $T_{G}^{\prime}$ envia pelo menos duas arestas de retorno para $I_{i_{0}}$.

Seja $x_{0}$ a raiz de $T_{G}^{\prime}$. Tem-se que os caminhos completos em $T_{G}$ até cada uma das folha em $F\left(T_{G}^{\prime}\right)$ são coincidentes desde a raiz de $T_{G}$ até pelo menos $x_{0}$; chame esse caminho entre a raiz e $x_{0}$ de $P$. Assim, todas as folhas em $F\left(T_{G}^{\prime}\right)$ compartilham dos mesmos ancestrais nos níveis a partir de $f_{i_{0}}\left(x^{\prime}\right)+1$, que são os vértices de $P$. Em particular, para os níveis em $I_{i_{0}}$, os ancestrais de qualquer folha em $F\left(T_{G}^{\prime}\right)$ são os $\left|I_{i_{0}}\right|=\left\lfloor 2^{f_{i_{0}}\left(x^{\prime}\right) / 4}\right\rfloor$ vértices do segmento de $P$ contido em $I_{i_{0}}$.

Considere uma folha $v \in F\left(T_{G}^{\prime}\right) \cap A_{i_{0}}$. Tem-se que $v$ envia pelo menos duas arestas de retorno para o segmento de $P$ em $I_{i_{0}}$. Conta-se o número de pares de vértices distintos nesse segmento; tem-se

$$
\left(\begin{array}{c}
\left\lfloor 2^{f_{i_{0}}\left(x^{\prime}\right) / 4}\right\rfloor \\
2
\end{array}\right)<2^{f_{i_{0}}\left(x^{\prime}\right) / 2-1}
$$

Cada $v \in F\left(T_{G}^{\prime}\right) \cap A_{i_{0}}$ será adjacente a pelo menos um desses pares. Mas o número de folhas 
em $F\left(T_{G}^{\prime}\right) \cap A_{i_{0}}$ é

$$
\left|F\left(T_{G}^{\prime}\right) \cap A_{i_{0}}\right| \geq 2^{-f_{i_{0}}\left(x^{\prime}\right) / 2}\left|F\left(T_{G}^{\prime}\right)\right|=2^{-f_{i_{0}}\left(x^{\prime}\right) / 2} 2^{f_{i_{0}}\left(x^{\prime}\right)+1}=2^{f_{i_{0}}\left(x^{\prime}\right) / 2+1} .
$$

Tem-se mais folhas em $F\left(T_{G}^{\prime}\right) \cap A_{i_{0}}$ do que pares de vértices contidos no segmento de $P$ em $I_{i_{0}}$. Assim, pelo menos duas folhas em $F\left(T_{G}^{\prime}\right) \cap A_{i_{0}}$ enviam arestas de retorno para um mesmo par de ancestrais em $I_{i_{0}}$. Isso é uma contradição, pois se forma um $C_{4}$.

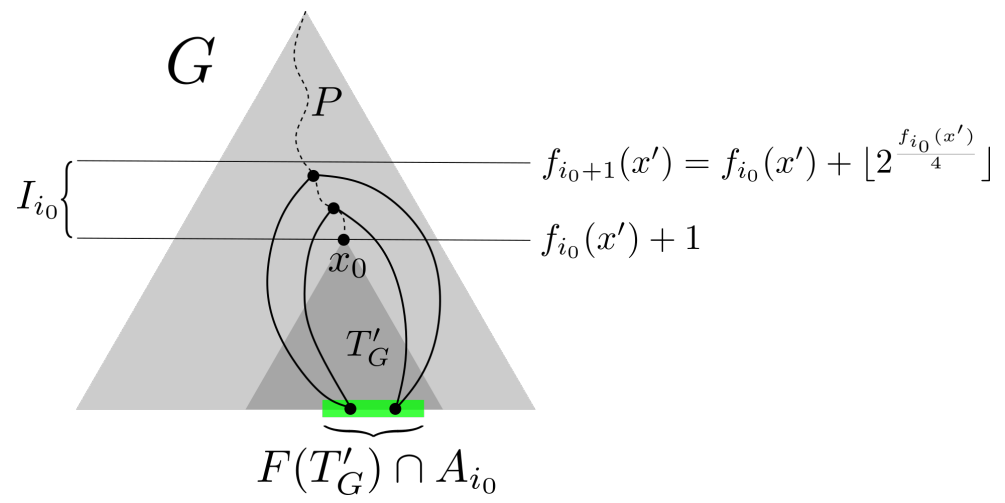

Figura 6.15: Há mais folhas em $F\left(T_{G}^{\prime}\right) \cap A_{i_{0}}$ do que pares de ancestrais a serem atingidos em $I_{i_{0}}$.

Agora se quer limitar $\left|\bigcup_{i \geq 0} A_{i}\right|$ para provar que $\left|\left(\bigcap_{i \geq 0} \overline{A_{i}}\right) \cap L_{G}\right|$, o número de vértices em $L_{G}$ que envia no máximo uma aresta de retorno em cada intervalo, é grande. Mais especificamente, que $\left|\left(\bigcap_{i \geq 0} \overline{A_{i}}\right) \cap L_{G}\right| \geq 2^{-f_{r-1}\left(x^{\prime}\right) / 8}\left|F\left(T_{G}\right)\right|$.

Provado isso, como cada vértice em $\left(\bigcap_{i \geq 0} \overline{A_{i}}\right) \cap L_{G}$ envia $r$ arestas de retorno para níveis acima de $x^{\prime}$ e não pode enviar duas num mesmo intervalo, tem-se que pelo menos um nível em $I_{r-1}$ ou acima deve ser atingido. Em outras palavras, toda folha em $\left(\bigcap_{i \geq 0} \overline{A_{i}}\right) \cap L_{G}$ envia pelo menos uma aresta para um nível acima de $f_{r-1}\left(x^{\prime}\right)$. Tomando $\ell^{\prime}=f_{r-1}\left(x^{\prime}\right)$, o conjunto $A_{G, \ell^{\prime}}=\left(\bigcap_{i \geq 0} \overline{A_{i}}\right) \cap L_{G}$ garante que $\ell^{\prime}$ é válido para $G$.

Como $f_{r-1}(y)$ é crescente, vale que $\ell^{\prime} \geq f_{r-1}(x)$. Assim, todo $G \in T B(x, r, g)$ possui um valor $\ell^{\prime}$ válido que é maior que ou igual a $f_{r-1}(x)$ e, portanto, $f_{r-1}(x) \in \mathbb{L}(x, r, g)$. Como $\ell(x, r, 4)$ é o máximo de $\mathbb{L}(x, r, g)$, conclui-se que $\ell(x, r, 4) \geq f_{r-1}(x)$.

Falta mostrar que $\left|\left(\bigcap_{i \geq 0} \overline{A_{i}}\right) \cap L_{G}\right| \geq 2^{-f_{r-1}\left(x^{\prime}\right) / 8}\left|F\left(T_{G}\right)\right|$. Para isso, basta que $\left(\sum_{i \geq 0}\left|A_{i}\right|\right) /\left|F\left(T_{G}\right)\right|<$ $2^{-x^{\prime} / 4-1}$, pois

$$
\begin{aligned}
& \left|\left(\bigcap_{i \geq 0} \overline{A_{i}}\right) \cap L_{G}\right| \geq\left|\bigcap_{i \geq 0} \overline{A_{i}}\right|+\left|L_{G}\right|-\left|F\left(T_{G}\right)\right| \\
& \geq\left(1-\frac{\left|\bigcup_{i \geq 0} A_{i}\right|}{\left|F\left(T_{G}\right)\right|}\right)\left|F\left(T_{G}\right)\right|+2^{-x^{\prime} / 4}\left|F\left(T_{G}\right)\right|-\left|F\left(T_{G}\right)\right| \\
& \geq\left(2^{-x^{\prime} / 4}-\frac{\sum_{i \geq 0}\left|A_{i}\right|}{\left|F\left(T_{G}\right)\right|}\right)\left|F\left(T_{G}\right)\right| \geq 2^{-x^{\prime} / 4-1}\left|F\left(T_{G}\right)\right| \\
& \geq 2^{-\left(x^{\prime}+\left\lfloor 2^{x^{\prime} / 4}\right\rfloor\right) / 8}\left|F\left(T_{G}\right)\right| \geq 2^{-f_{r-1}\left(x^{\prime}\right) / 8}\left|F\left(T_{G}\right)\right|
\end{aligned}
$$

onde a penúltima desigualdade vale, pois $x^{\prime} \geq 100$ e a última, pois $f_{r-1}\left(x^{\prime}\right) \geq f\left(x^{\prime}\right)$ para todo $r \geq 2$. 
Falta apenas verificar que $\left(\sum_{i \geq 0}\left|A_{i}\right|\right) /\left|F\left(T_{G}\right)\right|<2^{-x^{\prime} / 4-1}$. A partir de (6.5), tem-se

$$
\begin{aligned}
& \frac{\sum_{i \geq 0}\left|A_{i}\right|}{\left|F\left(T_{G}\right)\right|}<\sum_{i \geq 0} 2^{-f_{i}\left(x^{\prime}\right) / 2} \\
& =2^{-x^{\prime} / 2} \sum_{i \geq 0} 2^{-\left(f_{i}\left(x^{\prime}\right)-x^{\prime}\right) / 2}<2^{-x^{\prime} / 2} \sum_{k \geq 0} 2^{-k / 2} \\
& =2^{-x^{\prime} / 2}(2+\sqrt{2})<2^{-x^{\prime} / 4-1},
\end{aligned}
$$

onde a penúltima desigualdade vale, pois $f_{i}\left(x^{\prime}\right) \geq x^{\prime}$ para $i \geq 0$ e então $-\left(f_{i}\left(x^{\prime}\right)-x^{\prime}\right)$ percorre alguns inteiros negativos, enquanto $-k$ com $k \geq 0$ percorre todos. E a última desigualdade vale, pois $x^{\prime} \geq 100$.

Então $\ell(x, r, g)$ existe para $x \geq 100, r \geq 2$ e $g \geq 4$. Quando $g=4$, a iteração de $f$ resulta numa função Torre, $\ell(x, r, 4) \geq f_{r-1}(x) \geq 2 \uparrow^{2} r$; há mais detalhes disso no Teorema 6.3.10. Mas, para conseguir que $\ell(x, r, g)$ é muito grande, é necessário analisar o comportamento de $\ell(x, r, g)$ de forma mais específica, para outros valores de $g$. Assim, é buscada uma cota inferior maior para cada $\ell\left(x, r, 2^{s}\right)$, com $s \geq 2$.

Serão feitas duas induções, uma indução em $r$ no passo da indução em $s$. O caso base da indução em $s$, quando $g=2^{2}, r \geq 2$ e $x \geq 100$, é dado pelo Lema 6.3.7. Para o caso base da indução em $r$, quando $g=2^{s+1}, r=2$ e $x \geq 100$, usa-se o Lema 6.3.8.

Lema 6.3.8. Sejam $s \geq 2$ e $x \geq 100$ inteiros. Para todo $G \in T B\left(x, 2,2^{s+1}\right)$, existem $x^{\prime \prime} \geq x$ $e \ell^{\prime} \geq \ell\left(2^{x^{\prime \prime} / 4}, 2^{x^{\prime \prime} / 2}, 2^{s}\right)$ tal que $\ell^{\prime}$ é válido para $G$. Logo, $\ell\left(x, 2,2^{s+1}\right) \geq \ell\left(2^{x / 4}, 2^{x / 2}, 2^{s}\right)$.

Para o passo da indução em $s$ e base da indução em $r$, precisa-se comparar $\ell\left(x, 2,2^{s+1}\right) \operatorname{com} \ell\left(x_{0}, r_{0}, 2^{s}\right)$. Como $\ell$ é crescente, tem-se facilmente que $\ell\left(x, 2,2^{s+1}\right) \geq \ell\left(x, 2,2^{s}\right)$. Mas o Lema 6.3.8 consegue que $\ell\left(x, 2,2^{s+1}\right) \geq \ell\left(2^{x / 4}, 2^{x / 2}, 2^{s}\right)$, garantindo uma cota inferior maior.

O passo da indução em $r$ usa o Lema 6.3.9.

Lema 6.3.9. Sejam $s \geq 2, r \geq 3, x \geq 100$. Então $\ell\left(x, r, 2^{s+1}\right) \geq \ell\left(\ell\left(2^{x / 4}, 2^{x / 2}, 2^{s}\right), r-1,2^{s+1}\right)$.

Para o passo da indução em $r$, dentro da indução em $s$, precisa-se comparar $\ell\left(x, r, 2^{s+1}\right)$ com algum $\ell\left(x_{0}, r-1,2^{s+1}\right)$. O Lema 6.3 .9 consegue diminuir $r$ e iterar $\ell$ no parâmetro $x_{0}$. Essa iteração de $\ell$ e o fato do Lema 6.3.8 garantir uma boa base de indução, em que $r_{0}$ é uma função de $x$, resultará no comportamento Ackermanniano. Antes de se fazer as induções, seguem as demonstrações desses lemas.

Demonstração do Lema 6.3.8. Dado $G \in T B\left(x, 2,2^{s+1}\right)$ com árvore $T_{G}$, quer-se encontrar um $\ell^{\prime}$ válido para $G$. Para isso, é necessário que exista um subconjunto $A_{G, \ell^{\prime}}$ das folhas que seja suficientemente grande e em que cada folha envie pelo menos uma aresta para um nível acima de $\ell^{\prime}$. Para fazer a indução em $s$, quer-se tomar $\ell^{\prime} \geq \ell\left(x_{0}, r_{0}, 2^{s}\right)$, para certos valores $x_{0}$ e $r_{0}$. O que será feito é encontrar em $G$ um grafo auxiliar $F$ que seja um $T B\left(x_{0}, r_{0}, 2^{s}\right)$-grafo. O grafo $F$ estará relacionado com $G$ de forma que um valor $\ell^{\prime \prime} \geq \ell\left(x_{0}, r_{0}, 2^{s}\right)$ válido para $F$ determinará um valor $\ell^{\prime}$ válido para $G$.

Primeiro, são descritos os passos da demonstração sem atribuir valores a $x_{0}$ e $r_{0}$. Acompanhe a construção de $F$ com a Figura 6.16. O grafo auxiliar $F$ será construído sobre os vértices de $T_{G}$ cujos 


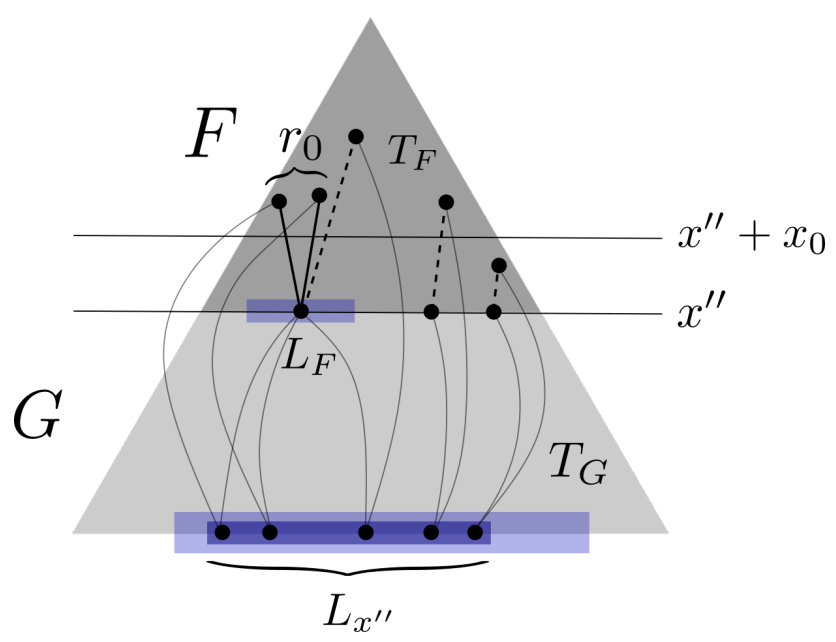

Figura 6.16: O grafo auxiliar $F$ estará contido em $G$.

níveis sejam pelo menos um certo valor $x^{\prime \prime}$. A árvore de $F$ será denotada $T_{F}$. Tem-se que $n_{F}(v)=$ $n_{G}(v)-x^{\prime \prime}$.

As arestas do grafo auxiliar $F$ vão cada uma corresponder a um caminho de comprimento 2 em $G$, de forma a garantir que não existirá circuito de comprimento menor que ou igual a $2^{s}$ em $F$. Toda folha de $L_{G}$ atinge exatamente dois ancestrais. A ideia é que as arestas de $F$ liguem esses dois ancestrais para certas folhas de $L_{G}$. As folhas de $F$ são os vértices no nível $x^{\prime \prime}$ de $T_{G}$ e as arestas de $F$ devem ter um dos extremos numa folha de $T_{F}$. Assim, consideram-se as folhas de $L_{G}$ cujo nível mais baixo atingido por suas arestas de retorno seja $x^{\prime \prime}$; esse conjunto será $L_{x^{\prime \prime}}$.

As arestas de $F$ ligarão os dois vizinhos de certas folhas em $L_{x^{\prime \prime}}$. Para $F$ ser um $T B\left(x_{0}, r_{0}, 2^{s}\right)$ grafo, não serão incluídas em $F$ arestas entre os vizinhos de folhas em $L_{x^{\prime \prime}}$ cujo nível mais alto atingido por suas arestas de retorno seja menor que ou igual a $x^{\prime \prime}+x_{0}$. Também não serão incluídas arestas para algumas outras folhas de $L_{x^{\prime \prime}}$, a fim de garantir que o grau de cada folha de $T_{F}$ que envia aresta de retorno seja exatamente $r_{0}$. Por fim, será preciso provar que o conjunto das folhas de $T_{F}$ que enviam arestas, $L_{F}$, é grande o suficiente. O tamanho de $L_{F}$ depende do tamanho de $L_{x^{\prime \prime}}$ e dos valores de $x_{0}$ e $r_{0}$ escolhidos.

Tendo definido $F$, descreve-se como encontrar $\ell^{\prime}$ válido para $G$. Uma primeira tentativa é a seguinte; acompanhe com a Figura 6.17. Como $F \in T B\left(x_{0}, r_{0}, 2^{s}\right)$, tem-se $\ell_{0} \geq \ell\left(x_{0}, r_{0}, 2^{s}\right)$ válido para $F$. Assim, encontra-se um conjunto $A_{F, \ell_{0}} \subset F\left(T_{F}\right)$ com $\left|A_{F, \ell_{0}}\right| \geq 2^{-\ell_{0} / 8}\left|F\left(T_{F}\right)\right|$ em que cada folha envia pelo menos uma aresta de retorno para um nível maior que $\ell_{0}$. Cada uma dessas aresta altas, que atingem um nível maior que $\ell_{0}$, corresponde a uma folha distinta em $T_{G}$, que tem uma aresta de retorno que atinge um nível maior que $x^{\prime \prime}+\ell_{0}$ em $G$. Assim, encontra-se em $F\left(T_{G}\right)$ um subconjunto $A_{G}$ de tamanho $\left|A_{G}\right| \geq 2^{-\ell_{0} / 8}\left|F\left(T_{F}\right)\right|$ em que cada folha envia pelo menos uma aresta para um nível maior que $x^{\prime \prime}+\ell_{0}$. As folhas de $F$ são os vértices de $T_{G}$ no nível $x^{\prime \prime}$, portanto $\left|F\left(T_{F}\right)\right|=2^{-x^{\prime \prime}}\left|F\left(T_{G}\right)\right|$ e $\left|A_{G}\right| \geq 2^{-\left(\ell_{0}+8 x^{\prime \prime}\right) / 8}\left|F\left(T_{G}\right)\right|$. Como $x^{\prime \prime}+\ell_{0}<8 x^{\prime \prime}+\ell_{0}$, o conjunto $A_{G}$ não é grande o suficiente para garantir que $x^{\prime \prime}+\ell_{0}$ seja válido para $G$.

Isso será remediado, subtraindo-se as 3 arestas de retorno mais altas de cada folha de $L_{F}$; obtendo-se outro grafo auxiliar $F^{\prime \prime} \in T B\left(x_{0}, r_{0}-3,2^{s+1}\right)$. Acompanhe com a Figura 6.18. Tomase $\ell^{\prime \prime} \geq \ell\left(x_{0}, r_{0}-3,2^{s+1}\right)$ e o conjunto $A_{F^{\prime \prime}, \ell^{\prime \prime}} \subset F\left(T_{F}\right)$ em que cada folha atinge um nível acima de $\ell^{\prime \prime}$ em $T_{F}$. Devolvendo as 3 arestas de retorno mais altas para as folhas em $A_{F^{\prime \prime}, \ell^{\prime \prime}}$, prova-se que uma grande parte delas terá uma aresta de retorno que atinge um nível $\ell^{\prime}>\ell^{\prime \prime}$; chame esse 


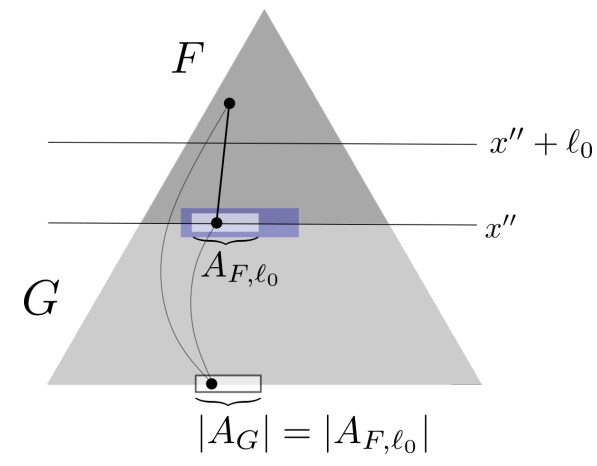

Figura 6.17: Tomando $\ell_{0} \geq \ell\left(x_{0}, r_{0}, 2^{s}\right)$, o tamanho de $A_{G}$ não é grande o suficiente.

subconjunto de $A_{F^{\prime \prime}, \ell^{\prime \prime}}^{\prime}$. Como $\ell^{\prime}$ é grande, valerá que $\left|A_{F^{\prime \prime}, \ell^{\prime \prime}}^{\prime}\right| \geq 2^{-\ell^{\prime} / 16}\left|F\left(T_{F}\right)\right|$. Em particular, como $A_{F^{\prime \prime}, \ell^{\prime \prime}}^{\prime} \subset A_{F^{\prime \prime}, \ell^{\prime \prime}}$, é necessário que $\ell^{\prime} \geq 2 \ell^{\prime \prime}$. O argumento para se encontrar $\ell^{\prime}$ é semelhante ao do Lema 6.3.7, em que se divide os níveis em intervalos e utiliza-se que não pode haver $C_{4}$. Para cada uma dessas arestas que atingem níveis acima de $\ell^{\prime}$, obtenha o conjunto de folhas de $T_{G}$ correspondente; chame de $A_{G}^{\prime \prime} \subset F\left(T_{G}\right)$. Assim, o tamanho de $A_{G}^{\prime \prime}$ será

$$
\left|A_{G}^{\prime \prime}\right|=\left|A_{F^{\prime \prime}, \ell^{\prime \prime}}^{\prime}\right| \geq 2^{-\ell^{\prime} / 16}\left|F\left(T_{F}\right)\right|=2^{-\left(\ell^{\prime}+16 x^{\prime \prime}\right) / 16}\left|F\left(T_{G}\right)\right| .
$$

Se $\ell^{\prime}$ for suficientemente grande, no caso $\ell^{\prime} \geq 16 x^{\prime}$, tem-se que

$$
\left|A_{G}^{\prime \prime}\right| \geq 2^{-\left(\ell^{\prime}+16 x^{\prime \prime}\right) / 16}\left|F\left(T_{G}\right)\right| \geq 2^{-\ell^{\prime} / 8}\left|F\left(T_{G}\right)\right| .
$$

Assim, o conjunto $A_{G}^{\prime \prime}$ garantirá que $\ell^{\prime}$ é válido para $G$.

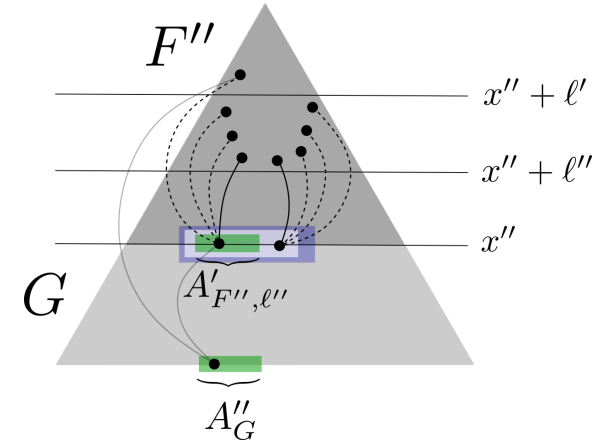

Figura 6.18: Reservam-se 3 arestas para garantir que níveis mais altos sejam atingidos e, assim, obter que $A_{G}^{\prime \prime}$ é grande suficiente.

Sabendo os passos da demonstração em termos gerais, no que se segue, ela será apresentada precisamente. Para obter $F \subset G$, define-se $x^{\prime \prime}$. Para $G$, tome $x^{\prime} \geq x$ e $L_{G}$ dados pela definição de $T B\left(x, 2,2^{s+1}\right)$. Assim, toda folha em $L_{G} \subset F\left(T_{G}\right)$ envia duas arestas de retorno para níveis acima de $x^{\prime} \mathrm{e}$

$$
\left|L_{G}\right| \geq 2^{-x^{\prime} / 4}\left|F\left(T_{G}\right)\right| .
$$

Considere, para cada $i>x^{\prime}$, o subconjunto $L_{i} \subset F\left(L_{G}\right)$ das folhas cujo nível mais baixo, dentre os 
atingidos por suas arestas de retorno, é exatamente $i$,

$$
L_{i}=\left\{v \in L_{G}: v u, v w \in E(G) \operatorname{com} n(u)=i, n(w)>i\right\} .
$$

O nível $x^{\prime \prime}$ é escolhido de modo que

$$
\left|L_{x^{\prime \prime}}\right| \geq \frac{1}{10} 2^{-x^{\prime \prime} / 4}\left|F\left(T_{G}\right)\right|
$$

Suponha que nenhum valor $i>x^{\prime}$ satisfaça (6.7). Como toda folha em $L_{G}$ envia duas arestas de retorno para níveis acima de $x^{\prime}$, tem-se

$$
\left|L_{G}\right|=\sum_{i>x^{\prime}}\left|L_{i}\right|<\frac{1}{10}\left|F\left(T_{G}\right)\right| \sum_{i>x^{\prime}} 2^{-i / 4}=\frac{1}{10}\left|F\left(T_{G}\right)\right| \frac{2^{-x^{\prime} / 4}}{1-2^{-1 / 4}} \leq \frac{\left|L_{G}\right|}{10\left(1-2^{-1 / 4}\right)}<\left|L_{G}\right|,
$$

onde a penúltima desigualdade vale por (6.6) e a última, pois $1-2^{-1 / 4}>10^{-1}$. Há uma contradição, o que significa que existe $x^{\prime \prime}>x^{\prime}$ que satisfaz (6.7).

Os vértices de $F$ são todos os vértices de $T_{G}$ com nível pelo menos $x^{\prime \prime}$. Para tomar as arestas de $F$, defina $x_{0}=2^{x^{\prime \prime}} / 4$ e $r_{0}=2^{x^{\prime \prime}} / 2+3$. As folhas em $L_{x^{\prime \prime}}$ que definirão arestas em $F$ precisam que suas arestas de retorno mais altas atinjam um nível acima de $x^{\prime \prime}+2^{x^{\prime \prime} / 4}$; chame esse subconjunto de $L^{\prime}$. Tem-se

$$
L^{\prime}=\left\{v \in L_{x^{\prime \prime}}: v u, v w \in E(G) \operatorname{com} n(u)=x^{\prime \prime}, n(w)>x^{\prime \prime}+2^{x^{\prime \prime} / 4}\right\}
$$

Ligando os vizinhos de qualquer folha em $L^{\prime}$ tem-se uma aresta de retorno em $F$ que atinge um nível acima de $2^{x^{\prime \prime} / 4}$ em $F$. Mas nem toda folha de $L^{\prime}$ irá determinar uma aresta de $F$, pois deseja-se que o grau de cada folha do conjunto $L_{F}$, das folhas que enviam arestas de retorno em $F$, seja precisamente $2^{x^{\prime \prime} / 2}+3$. Assim, tome o seguinte grafo $F^{\prime}$ que irá conter $F$ (Figura 6.19). Defina que

$$
\begin{aligned}
& V\left(F^{\prime}\right)=\left\{v \in V(G): n(v) \geq x^{\prime \prime}\right\} \mathrm{e} \\
& E\left(F^{\prime}\right)=\left\{\{u, w\} \in\left(\begin{array}{c}
V\left(F^{\prime}\right) \\
2
\end{array}\right): \exists v \in L^{\prime} \text { com } v u, v w \in E(G)\right\}
\end{aligned}
$$

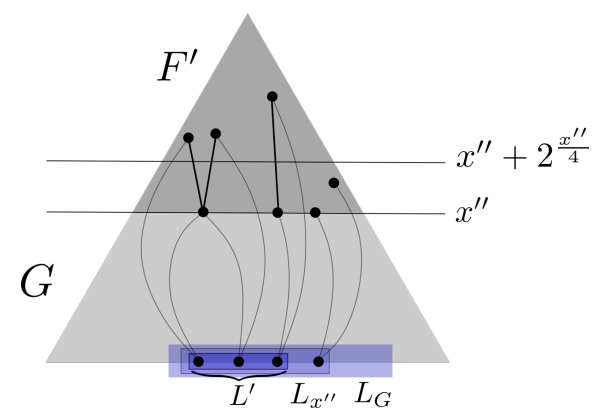

Figura 6.19: Primeiro, define-se o grafo auxiliar $F^{\prime}$.

Para provar que existe $F \in T B\left(2^{x^{\prime \prime} / 4}, 2^{x^{\prime \prime} / 2}+3,2^{s}\right)$ tal que $F \subset F^{\prime}$, é preciso encontrar um conjunto $L_{F} \subset F\left(T_{F}\right)$ tal que $\left|L_{F}\right| \geq 2^{-\left(2^{x^{\prime \prime} / 4}\right) / 4}\left|F\left(T_{F}\right)\right|$ em que cada folha tenha grau pelo me$\operatorname{nos} 2^{x^{\prime \prime} / 2}+3$. Isso será feito observando-se o número de arestas em $E\left(F^{\prime}\right)$ e o grau máximo que 
uma folha em $F\left(T_{F}\right)$ pode ter.

Note que $\left|E\left(F^{\prime}\right)\right|=\left|L^{\prime}\right|$. De fato, cada aresta de $E\left(F^{\prime}\right)$ se associa a uma folha em $L^{\prime}$ por definição. Por um lado, duas arestas distintas de $E\left(F^{\prime}\right)$ não podem estar associadas a um mesmo vértice de $L^{\prime}$, pois cada folha em $L^{\prime}$ tem apenas dois vizinhos, de modo que $\left|E\left(F^{\prime}\right)\right| \leq\left|L^{\prime}\right|$. Por outro lado, dois vértices distintos de $L^{\prime}$ não podem definir uma mesma aresta, senão haveria $C_{4} \subset G$, de modo que $\left|E\left(F^{\prime}\right)\right| \geq\left|L^{\prime}\right|$. Dessa forma, analisa-se o tamanho de $L^{\prime}$.

Considere $\overline{L^{\prime}}=L_{x^{\prime \prime}} \backslash L^{\prime}$, o conjunto das folhas de $T_{G}$ cuja aresta de retorno mais baixa atinge o nível $x^{\prime \prime}$ e a mais alta, um nível em $\left[x^{\prime \prime}+1, x^{\prime \prime}+\left\lfloor 2^{x^{\prime \prime} / 4}\right\rfloor\right]$. Tome $v \in \overline{L^{\prime}}$ e sejam $v u$ e $v w$ as arestas de retorno de $v$. Sem perda de generalidade, $u$ é tal que $n_{G}(u)=x^{\prime \prime}$. Quanto a $w$, ele é ancestral de $v$ e tem nível em $\left[x^{\prime \prime}+1, x^{\prime \prime}+\left\lfloor 2^{x^{\prime \prime}} / 4\right\rfloor\right]$, portanto está no caminho que começa no pai de $u$ e sobe mais $\left\lfloor 2^{x^{\prime \prime}} / 4\right\rfloor-1$ arestas em direção à raiz; são $\left\lfloor 2^{x^{\prime \prime}} / 4\right\rfloor$ possibilidades para $w$. Se $N\left(x^{\prime \prime}\right)$ é o número de vértices no nível $x^{\prime \prime}$, tem-se que existem no máximo $N\left(x^{\prime \prime}\right)\left\lfloor 2^{x^{\prime \prime}} / 4\right\rfloor$ pares de vizinhos possíveis para cada folha em $\overline{L^{\prime}}$. Como $\operatorname{cin}(G)>2^{s}$ e $s \geq 2$, duas folhas não podem compartilhar um mesmo par de vizinhos. Assim,

$$
\left|\overline{L^{\prime}}\right| \leq N\left(x^{\prime \prime}\right)\left\lfloor 2^{x^{\prime \prime} / 4}\right\rfloor \leq \frac{N\left(x^{\prime \prime}\right) 2^{x^{\prime \prime} / 4}}{2^{-x^{\prime \prime} / 4} \cdot 10^{-1} \cdot 2^{x^{\prime \prime}} N\left(x^{\prime \prime}\right)}\left|L_{x^{\prime \prime}}\right|=10 \cdot 2^{-x^{\prime \prime} / 2}\left|L_{x^{\prime \prime}}\right|
$$

onde a segunda desigualdade vale por (6.7).

Dessa forma, vale que

$$
\begin{aligned}
\left|L^{\prime}\right| & \geq\left(1-10 \cdot 2^{-x^{\prime \prime} / 2}\right)\left|L_{x^{\prime \prime}}\right| \geq\left(\frac{1}{10 \cdot 2^{x^{\prime \prime} / 4}}-\frac{1}{2^{3 x^{\prime \prime} / 4}}\right)\left|F\left(T_{G}\right)\right| \\
& =2^{-x^{\prime \prime} / 4}\left(\frac{1}{10}-\frac{1}{2^{x^{\prime \prime} / 2}}\right)\left|F\left(T_{G}\right)\right| \geq \frac{1}{11} 2^{-x^{\prime \prime} / 4}\left|F\left(T_{G}\right)\right|
\end{aligned}
$$

onde a última desigualdade vale para $x^{\prime \prime} \geq 100$.

Sabendo o número de arestas $\left|E\left(F^{\prime}\right)\right|$, pode-se calcular o grau médio de uma folha de $T_{F}$, que é

$$
d=\frac{\left|L^{\prime}\right|}{\left|F\left(T_{F}\right)\right|} \geq \frac{2^{-x^{\prime \prime} / 4}}{11} \frac{\left|F\left(T_{G}\right)\right|}{\left|F\left(T_{F}\right)\right|}=\frac{2^{3 x^{\prime \prime} / 4}}{11}
$$

onde (6.8) foi usado na primeira desigualdade e, depois, que $\left|F\left(T_{G}\right)\right|=2^{x^{\prime \prime}}\left|F\left(T_{F^{\prime}}\right)\right|$.

Assim, tem-se

$$
\frac{d}{2} \geq \frac{2^{3 x^{\prime \prime} / 4}}{22} \geq 2^{x^{\prime \prime} / 2}+3
$$

onde a última desigualdade vale, pois $x^{\prime \prime} \geq 100$. Tome o conjunto das folhas com grau pelo me$\operatorname{nos} d / 2$,

$$
L_{\geq d / 2}=\left\{u \in F\left(T_{F}\right): d(u) \geq \frac{d}{2}\right\}
$$

Para que $L_{\geq d / 2}$ seja $L_{F}$, basta provar que ele tem tamanho $\left|L_{\geq d / 2}\right| \geq 2^{-\left(2^{x^{\prime \prime} / 4}\right) / 4}\left|F\left(T_{F}\right)\right|$.

Para contar quantas folhas de $T_{F}$ precisam ter grau pelo menos $d / 2$ de modo a garantir grau médio $d$, é preciso observar o grau máximo que uma folha pode ter. Se uma aresta em $E\left(F^{\prime}\right)$ tem extremo em $u \in F\left(T_{F}\right)$, deve existir alguma folha de $T_{G}$ com uma aresta de retorno que atinja $u$. Arestas de retorno só podem atingir ancestrais, então somente as folhas da subárvore de $T_{G}$ com raiz 
em $u$ poderiam determinar arestas em $E\left(F^{\prime}\right)$ com extremo em $u$. O número de folhas na subárvore com raiz $u$ é $2^{x^{\prime \prime}}$, pois $n_{G}(u)=x^{\prime \prime}$. Como cada aresta de $E\left(F^{\prime}\right)$ corresponde a uma folha diferente de $T_{G}$, tem-se que o grau de $u$ em $F^{\prime}$, denotado por $d_{F^{\prime}}(u)$, é no máximo $2^{x^{\prime \prime}}$.

Escreva o grau médio das folhas em função dos graus de cada folha. Seja $L_{<d / 2}=F\left(T_{F}\right) \backslash L_{\geq d / 2}$, o conjunto das folhas com grau menor que $d / 2$. Tem-se

$$
d=\frac{\sum_{u \in L_{\geq d / 2}} d_{F^{\prime}}(u)+\sum_{u \in L_{<d / 2}} d_{F^{\prime}}(u)}{\left|F\left(T_{F}\right)\right|} \leq \frac{2^{x^{\prime \prime}}\left|L_{\geq d / 2}\right|+(d / 2)\left|F\left(T_{F}\right)\right|}{\left|F\left(T_{F}\right)\right|} .
$$

Isolando a fração buscada, tem-se

$$
\frac{\left|L_{\geq d / 2}\right|}{\left|F\left(T_{F}\right)\right|} \geq \frac{d}{2^{x^{\prime \prime}+1}} \geq \frac{2^{-x^{\prime \prime} / 4}}{22} \geq 2^{-\left(2^{x^{\prime \prime} / 4}\right) / 4}
$$

onde a segunda desigualdade vale por (6.9) e a última, pois $x^{\prime \prime} / 4$ é muito menor que $\left(2^{x^{\prime \prime} / 4}\right) / 4$ quando $x^{\prime \prime}$ é grande. Dessa forma, encontra-se $L_{F}=L_{\geq d / 2}$. Pode-se definir as arestas de $F$. Tem-se que $E(F)$ serão apenas arestas de $E\left(F^{\prime}\right)$ que tenham um extremo em $L_{F}$. Além disso, para cada folha em $L_{F}$, são escolhidas exatamente $2^{x^{\prime \prime} / 2}+3$ arestas de retorno.

Uma última observação para garantir que $F \in T B\left(2^{x^{\prime \prime} / 4}, 2^{x^{\prime \prime} / 2}+3,2^{s}\right)$ é que $\operatorname{cin}(F)>2^{s}$. Seja $u w \in E(F)$ uma aresta de um circuito em $F$ com comprimento $k$. Encontre $v \in L^{\prime}$ que corresponda a $u w$, isto é, tal que existem $v u, v w \in E(G)$. Substitua a aresta $u w$ por $(u, v, w)$. Como dito, cada aresta de $E(F)$ se associa a um vértice distinto de $L^{\prime}$, pois cada folha de $L^{\prime}$ envia apenas duas arestas de retorno. Desta forma, tem-se um circuito em $G$ cujo comprimento é $2 k$. Pela cintura de $G$, vale que $2 k>2^{s+1}$, então $k>2^{s}$.

O último passo da demonstração é remover as 3 arestas de retorno mais altas de cada folha em $L_{F}$ e encontrar $\ell^{\prime}$ válido para $G$. Assim, remova essas 3 arestas e chame o grafo obtido de $F^{\prime \prime}$. O mesmo $L_{F}=L_{F^{\prime \prime}}$ garante que $F^{\prime \prime}$ é um $T B\left(2^{x^{\prime \prime} / 4}, 2^{x^{\prime \prime} / 2}, 2^{s}\right)$. Tome $\ell^{\prime \prime} \geq \ell\left(2^{x^{\prime \prime} / 4}, 2^{x^{\prime \prime} / 2}, 2^{s}\right)$ válido para $F^{\prime \prime}$. Isto é, existe $A_{F^{\prime \prime}, \ell^{\prime \prime}} \subset F\left(T_{F}\right)$ com $\left|A_{F^{\prime \prime}, \ell^{\prime \prime}}\right| \geq 2^{\ell^{\prime \prime}} / 8\left|F\left(T_{F}\right)\right|$ e cujas folhas enviam pelo menos uma aresta de retorno cada para um nível maior que $\ell^{\prime \prime}$.

Devolvendo as 3 arestas mais altas para cada vértice de $A_{F^{\prime \prime}, \ell^{\prime \prime}}$, será encontrado um valor $\ell^{\prime}$ que será válido para $G$. Cada folha em $A_{F^{\prime \prime}, \ell^{\prime \prime}}$ tem alguma aresta de retorno em $E\left(F^{\prime \prime}\right)$ que atinge um nível maior que $\ell^{\prime \prime}$. Assim, devolvendo as 3 arestas mais altas, elas também atingirão níveis acima de $\ell^{\prime \prime}$. Será feito o mesmo que na demonstração do Lema 6.3.7. Particione os níveis acima de $\ell^{\prime \prime}$ em intervalos

$$
I_{i}=\left[f_{i}\left(\ell^{\prime \prime}\right)+1, f_{i+1}\left(\ell^{\prime \prime}\right)\right],
$$

para $i \geq 0$, onde $f(y)=y+\left\lfloor 2^{y / 4}\right\rfloor$. Defina

$$
B_{i} \subset F\left(T_{F}\right)=\left\{v \in A_{F^{\prime \prime}, \ell^{\prime \prime}}: \exists v u_{1} \neq v u_{2} \in E(F) \backslash E\left(F^{\prime \prime}\right) \operatorname{com} n_{F}\left(u_{1}\right), n_{F}\left(u_{2}\right) \in I_{i}\right\},
$$

o conjunto das folhas em $A_{F^{\prime \prime}, \ell^{\prime \prime}}$ tais que pelo menos duas das suas 3 arestas mais altas atingem níveis dentro de um mesmo intervalo $I_{i}$. Pelo mesmo argumento da demonstração do Lema 6.3.7, que conta o número de pares de ancestrais possíveis dentro de um mesmo nível e usa que não pode haver $C_{4}$, tem-se

$$
\left|B_{i}\right|<2^{-f_{i}\left(\ell^{\prime \prime}\right) / 2}\left|F\left(T_{F}\right)\right|
$$


Ainda seguindo a demonstração do Lema 6.3.7, para mostrar que uma fração grande das folhas em $A_{F^{\prime \prime}, \ell^{\prime \prime}}$ envia cada uma das 3 arestas extras para níveis em intervalos diferentes, calcula-se que

$$
\frac{\sum_{i \geq 0}\left|B_{i}\right|}{\left|F\left(T_{F}\right)\right|} \leq 2^{-\ell^{\prime \prime} / 2}(2+\sqrt{2}) \leq 2^{-\ell^{\prime \prime} / 8-1}
$$

onde a última desigualdade vale, pois $\ell^{\prime \prime} \geq 2^{x^{\prime \prime} / 4} \geq x \geq 100$.

Seja $\overline{B_{i}}=F\left(T_{F}\right)-B_{i}$. Tem-se que $\bigcap_{i \geq 0} \overline{B_{i}}$ contém as folhas em $A_{F^{\prime \prime}, \ell^{\prime \prime}}$ cujas 3 arestas extras atingem níveis em três intervalos distintos, mas ele também contém as folhas fora de $A_{F^{\prime \prime}, \ell^{\prime \prime}}$, que não receberam as 3 arestas de volta. Assim, para saber a fração de $F\left(T_{F}\right)$ cujas arestas extras não repetem intervalos, calcula-se

$$
\begin{aligned}
& \left|\left(\bigcap_{i \geq 0} \overline{B_{i}}\right) \cap A_{F^{\prime \prime}, \ell^{\prime \prime}}\right| \geq\left|\bigcap_{i \geq 0} \overline{B_{i}}\right|+\left|A_{F^{\prime \prime}, \ell^{\prime \prime}}\right|-\left|F\left(T_{F}\right)\right| \\
& \geq\left(1-\frac{\left|\bigcup_{i \geq 0} B_{i}\right|}{\left|F\left(T_{F}\right)\right|}\right)\left|F\left(T_{F}\right)\right|+2^{-\ell^{\prime \prime} / 8}\left|F\left(T_{F}\right)\right|-\left|F\left(T_{F}\right)\right| \\
& \geq\left(2^{-\ell^{\prime \prime} / 8}-\frac{\sum_{i \geq 0}\left|B_{i}\right|}{\left|F\left(T_{F}\right)\right|}\right)\left|F\left(T_{F}\right)\right| \geq 2^{-\ell^{\prime \prime} / 8-1}\left|F\left(T_{F}\right)\right| \\
& \geq 2^{-\left(\ell^{\prime \prime}+\left\lfloor 2^{\ell^{\prime \prime} / 4}\right\rfloor\right) / 16}\left|F\left(T_{F}\right)\right| \geq 2^{-f_{2}\left(\ell^{\prime \prime}\right) / 16}\left|F\left(T_{F}\right)\right| .
\end{aligned}
$$

Além disso, se as 3 arestas extras das folhas em $\left(\bigcap_{i \geq 0} \overline{B_{i}}\right) \cap A_{F^{\prime \prime}, \ell^{\prime \prime}}$ atingem cada uma um nível em um intervalo distinto, a mais alta delas atinge um nível maior que $\ell^{\prime}=f_{2}\left(\ell^{\prime \prime}\right)$ (Figura 6.20). O conjunto $A_{F^{\prime \prime}, \ell^{\prime \prime}}^{\prime}$ definido no início da demonstração, das folhas em $A_{F^{\prime \prime}, \ell^{\prime \prime}}$ tais que uma das 3 arestas extras atinge um nível acima de $\ell^{\prime}$ é $\left(\bigcap_{i \geq 0} \overline{B_{i}}\right) \cap A_{F^{\prime \prime}, \ell^{\prime \prime}}$.

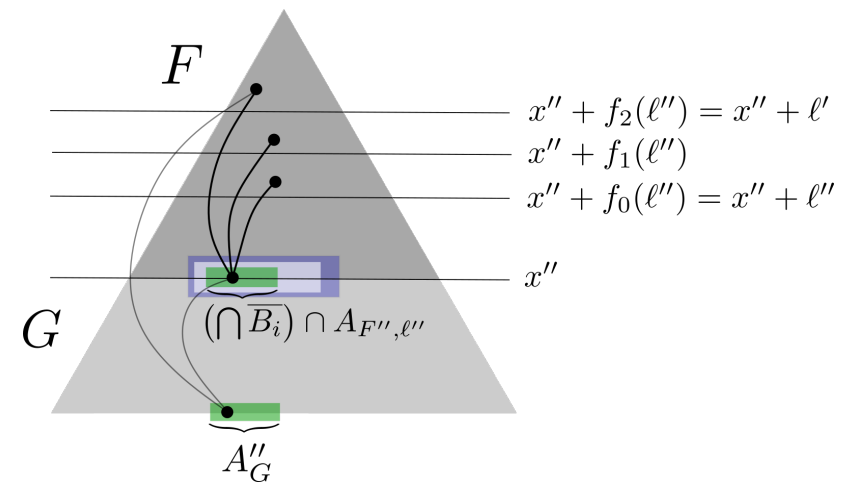

Figura 6.20: Devolvendo as arestas extras a $A_{F^{\prime \prime}, \ell^{\prime \prime}}$, encontra-se que boa parte de $F\left(T_{F}\right)$ envia arestas acima de um nivel mais alto $\ell^{\prime}>\ell^{\prime \prime}$.

Para provar que $\ell^{\prime}$ é válido para $G$ é preciso encontrar um $A_{G, \ell^{\prime}}$. Defina o conjunto $A_{G}^{\prime \prime} \subset F\left(T_{G}\right)$ da seguinte forma. Para cada $u \in\left(\bigcap_{i \geq 0} \overline{B_{i}}\right) \cap A_{F^{\prime \prime}, \ell^{\prime \prime}}$, escolha uma aresta $u w \in E(F) \operatorname{com} n_{F}(w)>$ $\ell^{\prime}=f_{2}\left(\ell^{\prime \prime}\right)$. Pela forma que as arestas de $E(F)$ são definidas, existe $v \in L^{\prime} \subset F\left(T_{G}\right)$ tal que vu e $v w$ são arestas de $E(G)$. Coloque $v$ em $A_{G}^{\prime \prime}$.

Note que cada folha em $A_{G}^{\prime \prime}$ envia pelo menos uma aresta para um nível maior que $x^{\prime \prime}+\ell^{\prime} \geq \ell^{\prime}$. Além disso, tem-se uma folha distinta em $A_{G}^{\prime \prime}$ para cada vértice de $\left(\bigcap_{i \geq 0} \overline{B_{i}}\right) \cap A_{F^{\prime \prime}, \ell^{\prime \prime}}$, pois já foi notado que arestas diferentes de $E(F)$ sempre estão associadas a folhas diferentes de $F\left(T_{G}\right)$. Dessa 
forma,

$$
\left|A_{G}^{\prime \prime}\right|=\left|\left(\bigcap_{i \geq 0} \overline{B_{i}}\right) \cap A_{F^{\prime \prime}, \ell^{\prime \prime}}\right| \geq 2^{-f_{2}\left(\ell^{\prime \prime}\right) / 16}\left|F\left(T_{F}\right)\right| \geq 2^{-\ell^{\prime} / 16} 2^{-x^{\prime \prime}}\left|F\left(T_{G}\right)\right| \geq 2^{-\ell^{\prime} / 8}\left|F\left(T_{G}\right)\right|,
$$

a última desigualdade vale pois $\ell^{\prime} \geq \ell^{\prime \prime} \geq 2^{x^{\prime \prime} / 4}$ e $x^{\prime \prime} \geq x \geq 100$.

Desta forma, encontra-se $\ell^{\prime} \geq \ell^{\prime \prime} \geq \ell\left(2^{x^{\prime \prime}} / 4,2^{x^{\prime \prime}} / 2,2^{s}\right)$ válido para $G$. Pela Proposição 6.3.6, tem-se que $\ell\left(2^{x^{\prime \prime} / 4}, 2^{x^{\prime \prime} / 2}, 2^{s}\right) \geq \ell\left(2^{x / 4}, 2^{x / 2}, 2^{s}\right)$. Assim, $\ell\left(2^{x / 4}, 2^{x / 2}, 2^{s}\right) \in \mathbb{L}\left(x, 2,2^{s+1}\right)$ e conclui-se que $\ell\left(x, 2,2^{s+1}\right) \geq \ell\left(2^{x / 4}, 2^{x / 2}, 2^{s}\right)$.

Para a indução em $r$, tem-se o Lema 6.3.9.

Demonstração do Lema 6.3.9. A afirmação que se quer provar é que, para $s \geq 2, r \geq 3$ e $x \geq 100$, vale que

$$
\ell\left(x, r, 2^{s+1}\right) \geq \ell\left(\ell\left(2^{x / 4}, 2^{x / 2}, 2^{s}\right), r-1,2^{s+1}\right) .
$$

Segundo o Lema $6.3 .8, \ell\left(x, 2,2^{s+1}\right) \geq \ell\left(2^{x / 4}, 2^{x / 2}, 2^{s}\right)$. Assim, como $\ell$ é crescente, basta provar que

$$
\ell\left(x, r, 2^{s+1}\right) \geq \ell\left(\ell\left(x, 2,2^{s+1}\right), r-1,2^{s+1}\right) .
$$

Para cada $G \in T B\left(x, r, 2^{s+1}\right)$ com árvore $T_{G}$, encontra-se um subgrafo $F \in T B\left(\ell\left(x, 2,2^{s+1}\right), r-\right.$ $\left.1,2^{s+1}\right)$ com árvore $T_{F}=T_{G}$. Por um argumento como o da demonstração da Propriedade 6.3.6, obtém-se que $\ell\left(x, r, 2^{s+1}\right) \geq \ell\left(\ell\left(x, 2,2^{s+1}\right), r-1,2^{s+1}\right)$.

Assim, procura-se $F \subset G$. O grafo $F$ deve ter um subconjunto das folhas $L_{F}$, em que cada folha envie pelo menos $r-1$ arestas de retorno para níveis acima de certo $x^{\prime \prime} \geq \ell\left(x, 2,2^{s+1}\right)$ e que seja grande, $\left|L_{F}\right| \geq 2^{-x^{\prime \prime} / 4}\left|F\left(T_{G}\right)\right|$. O que será feito é encontrar um subconjunto das folhas em $F\left(T_{G}\right)$ que tenha certas propriedades que garantirão que ele será um tal $L_{F}$ em $F$. Para isso, utiliza-se um $F^{\prime} \in T B\left(x, 2,2^{s+1}\right)$ contido em $G$.

Como $G \in T B\left(x, r, 2^{s+1}\right)$, pode-se tomar $x^{\prime} \geq x$ e $L_{G}$ como na definição de $T B\left(x, r, 2^{s+1}\right)$. Para definir o subgrafo $F^{\prime}$, basta escolher duas arestas de retorno para cada folha de $L_{G}$; serão escolhidas as duas arestas mais baixas. Os mesmos $x^{\prime}$ e $L_{F^{\prime}}=L_{G}$ garantem que $F^{\prime} \in T B\left(x, 2,2^{s+1}\right)$. Assim, o subgrafo $F^{\prime}$ tem os mesmos vértices que $G$ e suas arestas são apenas as duas mais baixas de cada folha em $L_{G}$ (Figura 6.21).

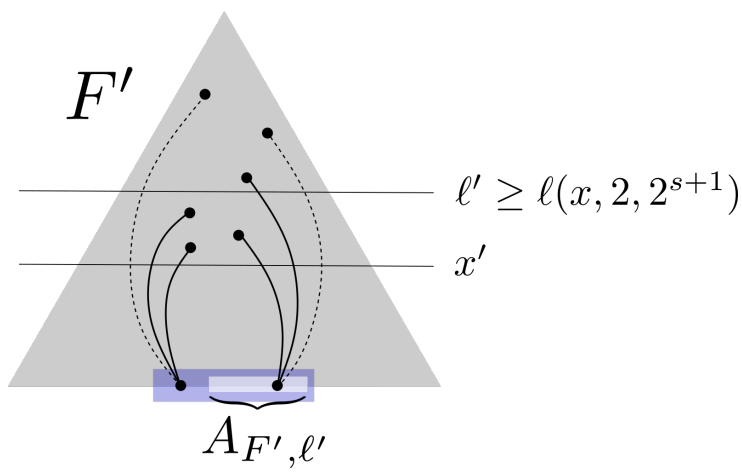

Figura 6.21: Deixe apenas as duas arestas mais baixas de cada folha para definir $F^{\prime} \subset G$.

Como $\ell\left(x, 2,2^{s+1}\right) \in \mathbb{L}\left(x, 2,2^{s+1}\right)$, existem $\ell^{\prime} \geq \ell\left(x, 2,2^{s+1}\right)$ e $A_{F^{\prime}, \ell^{\prime}} \subset F\left(T_{G}\right)$ tais que toda folha em $A_{F^{\prime}, \ell^{\prime}}$ envia pelo menos uma aresta para um nível acima de $\ell^{\prime}$ e $\left|A_{F^{\prime}, \ell^{\prime}}\right| \geq 2^{-\ell^{\prime} / 8}\left|F\left(T_{G}\right)\right| \geq$ 
$2^{-\ell^{\prime} / 4}\left|F\left(T_{G}\right)\right|$. Isso significa que, em $F^{\prime}$, pelo menos a aresta mais alta de cada folha em $A_{F^{\prime}, \ell^{\prime}}$ atinge um nível acima de $\ell^{\prime}$. Como as arestas de $F^{\prime}$ são as duas mais baixas de cada folha em $L_{G}$, tem-se que, em $G$, para cada folha em $A_{F^{\prime}, \ell^{\prime}}$, as arestas que incidem nela, excluindo-se a mais baixa, atingem níveis acima de $\ell^{\prime}$.

Tome $L_{F}=A_{F^{\prime}, \ell^{\prime}}$ e construa o subgrafo $F$ a partir de $G$ removendo as arestas que não incidem em $L_{F}$ e a aresta mais baixa para cada folha em $L_{F}$ (Figura 6.22). Assim, em $F$, cada folha em $L_{F}$ envia $r-1$ arestas para níveis acima de $\ell^{\prime} \geq \ell\left(x, 2,2^{s+1}\right)$ e $\left|L_{F}\right| \geq 2^{-\ell^{\prime} / 4}\left|F\left(T_{F}\right)\right|$. Temse $F \in T B\left(\ell\left(x, 2,2^{s+1}\right), r-1,2^{s+1}\right)$ contido em $G$ e com $T_{F}=T_{G}$.

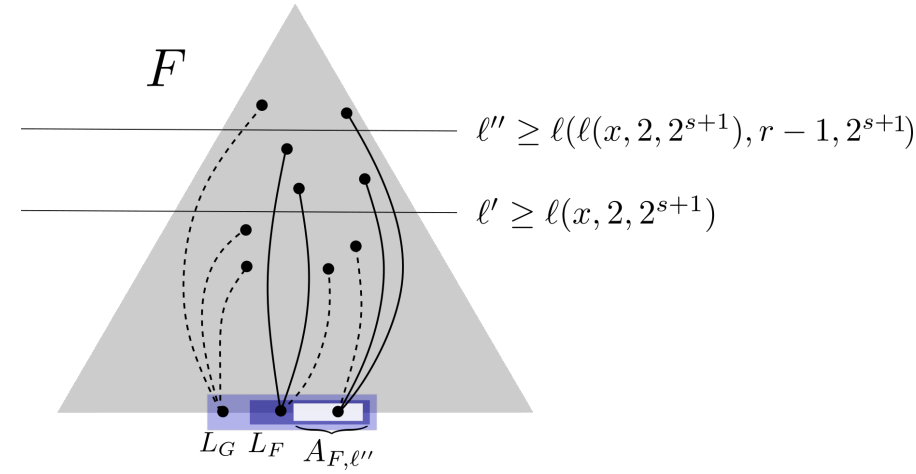

Figura 6.22: Encontra-se $F \in T B\left(\ell\left(x, 2,2^{s+1}\right), r-1,2^{s+1}\right)$ em $G$.

Assim, para qualquer $G \in T B\left(x, r, 2^{s+1}\right)$, encontra-se $F \in T B\left(\ell\left(x, 2,2^{s+1}\right), r-1,2^{s+1}\right)$. Dessa forma, tem-se $\ell^{\prime \prime} \geq \ell\left(\ell\left(x, 2,2^{s+1}\right), r-1,2^{s+1}\right)$ e $A_{G, \ell^{\prime \prime}}=A_{F, \ell^{\prime \prime}} \subset F\left(T_{G}\right)$, com $\left|A_{G, \ell^{\prime \prime}}\right| \geq 2^{-\ell^{\prime \prime} / 8}\left|F\left(T_{G}\right)\right|$, cujas folhas que enviam pelo menos uma aresta para um nível acima de $\ell^{\prime \prime}$. O valor $\ell^{\prime \prime}$ é válido para $G$ e $\ell\left(\ell\left(x, 2,2^{s+1}\right), r-1,2^{s+1}\right) \in \mathbb{L}\left(x, r, 2^{s+1}\right)$. Logo, $\ell\left(x, r, 2^{s+1}\right) \geq \ell\left(\ell\left(x, 2,2^{s+1}\right), r-1,2^{s+1}\right)$.

Com os lemas 6.3.7, 6.3.8 e 6.3.9, uma cota inferior Ackermanniana pode ser obtida para $\ell(x, r, g)$.

Teorema 6.3.10. Para valores $x \geq 100, r \geq 2$ e $s \geq 2$, tem-se $\ell\left(x, r, 2^{s}\right) \geq 2 \uparrow s r$.

Demonstração. A prova é feita por indução. Primeiro, calcule o caso $s=2$. Sejam $x \geq 100$ e $r \geq 2$, quer-se mostrar que $\ell(x, r, 4) \geq 2 \uparrow^{2} r$.

Segundo o Lema 6.3.7,

$$
\ell(x, r, 4) \geq f_{r-1}(x), \text { onde } f(y)=y+\left\lfloor 2^{y / 4}\right\rfloor .
$$

Veja alguns casos em que $r$ é pequeno,

$$
\ell(x, 2,4) \geq f_{1}(x) \geq x-1+2^{x / 4} \geq 2^{x / 4} \geq 2^{2}=2 \uparrow^{2} 2 .
$$

Agora, para $r=3$, tem-se

$$
\ell(x, 3,4) \geq f_{2}(x) \geq 2^{f(x) / 4} \geq 2^{2^{x / 4-2}} \geq 2^{2^{2}}=2 \uparrow^{2} 3 .
$$

Foi visto que $f_{2}(x) \geq 2^{2^{x / 4-2}}$. Prova-se por indução que, para todo $i \geq 2$, tem-se

$$
f_{i}(x) \geq \underbrace{2^{\cdot \cdot^{x / 4-2}}}_{\mathrm{i}+1} .
$$


Seja $i \geq 2$ e suponha por hipótese de indução que a cota é válida para $f_{i}(x)(x \geq 100)$. Agora,

$$
f_{i+1}(x)=f_{i}(f(x)) \geq \underbrace{2^{2^{f(x) / 4-2}}}_{\mathrm{i}+1} .
$$

No expoente, tem-se

$$
\frac{f(x)}{4}-2 \geq \frac{x-1+2^{x / 4}}{4}-2 \geq \frac{x}{4}-\frac{9}{4}+\frac{2^{x / 4}}{4} \geq 2^{x / 4-2} .
$$

Portanto,

$$
f_{i+1}(x) \geq \underbrace{2^{2^{2^{x / 4-2}}}}_{\mathrm{i}+2}
$$

o que completa a indução em $i$.

Dessa forma, para $r \geq 3$, pode-se calcular

$$
\ell(x, r, 4) \geq f_{r-1}(x) \geq \underbrace{2 \cdot \cdot^{2^{x / 4-2}}}_{\mathrm{r}} \geq \underbrace{2^{\cdot} \cdot \cdot^{2^{2}}}_{\mathrm{r}}=2 \uparrow^{2} r .
$$

Tem-se a base para a indução em $s$.

Seja $s \geq 2$ e suponha que para $x \geq 100$ e $r \geq 2$, vale que $\ell\left(x, r, 2^{s}\right) \geq 2 \uparrow^{s} r$. Agora, pelo Lema 6.3 .8 e pela hipótese, tem-se

$$
\ell\left(x, 2,2^{s+1}\right) \geq \ell\left(2^{x / 4}, 2^{x / 2}, 2^{s}\right) \geq 2 \uparrow^{s} 2^{x / 2} .
$$

Essa é a base para mais uma indução, agora em $r$.

Seja $r \geq 2$ e suponha que para $x \geq 100$, vale que $\ell\left(x, r, 2^{s+1}\right) \geq \underbrace{2 \uparrow^{s} 2 \uparrow^{s} \ldots \uparrow^{s} 2 \uparrow}_{\# 2=r} \uparrow^{(x / 2)}$.

Para o passo da indução, use o Lema 6.3.9

$$
\ell\left(x, r+1,2^{s+1}\right) \geq \ell\left(\ell\left(2^{x / 4}, 2^{x / 2}, 2^{s}\right), r, 2^{s+1}\right) .
$$

Pela hipótese da indução em $s$, tem-se

$$
\ell\left(x, r+1,2^{s+1}\right) \geq \ell\left(2 \uparrow^{s} 2^{x / 2}, r, 2^{s+1}\right) .
$$

Finalmente, pela hipótese da indução em $r$, vale que

$$
\ell\left(x, r+1,2^{s+1}\right) \geq \underbrace{2 \uparrow^{s} 2 \uparrow^{s} \ldots \uparrow^{s} 2}_{\mathrm{r}} \uparrow \frac{2 \uparrow^{s} 2^{x / 2}}{2} .
$$

Como $2^{y / 2} \geq y$ (desde que $y \geq 4$ ), então tomando $y=2 \uparrow^{s} 2^{x / 2}$,

$$
\ell\left(x, r+1,2^{s+1}\right) \geq \underbrace{2 \uparrow^{s} 2 \uparrow^{s} \ldots \uparrow^{s}\left(2 \uparrow^{s} 2 \uparrow\right.}_{r+1} \frac{x}{2}),
$$

terminando a indução em $r$. 
O passo da indução em $s$ pode ser completado. Seja $x \geq 100$ e $r \geq 2$,

$$
\ell\left(x, r, 2^{s+1}\right) \geq \underbrace{2 \uparrow^{s} \ldots \uparrow^{s} 2}_{r} \uparrow^{x} \geq 2 \uparrow^{s+1} r .
$$

Já foi observado que a altura mínima da arvore de um $T B(x, r, g)$-grafo é pelo menos $\ell(x, r, g)$. Também foi dito que os TB(x,r,g)-grafos seriam utilizados para se obter uma cota inferior para a altura mínima $m\left(2, r^{\prime}, g^{\prime}\right)$ da árvore de um $\left(2, r^{\prime}, g^{\prime}\right)$-grafo. Para isso, falta apenas verificar como $m\left(2, r^{\prime}, g^{\prime}\right)$, se relaciona com $\ell(x, r, g)$. Será provada a seguinte relação.

Teorema 6.3.11. Sejam $g>102$ par e $r \geq 1$ inteiro, vale que $m(2, r, g) \geq 1+\ell(100,2 r, g / 2-1)$.

Demonstração. Sejam $g>102$ par, $r \geq 1$ inteiro e $G$ um $(2, r, g)$-grafo cuja árvore $T_{G}$ tem altura $m(2, r, g)$. A partir de $G$, construa $F \in T B(100,2 r, g / 2-1)$ (Figura 6.23). A árvore de $F$ será $T_{F}=T_{G} \backslash F\left(T_{G}\right)$, a árvore de $G$ subtraída das folhas. Tem-se que $T_{F}$ é binária completa e tem altura $m(2, r, g)-1$.

Para cada folha $v \in F\left(T_{F}\right)$, sejam $u$ e $w$ seus dois filhos em $T_{G}$. Como $G$ é um $(2, r, g)$-grafo, toda folha de $T_{G}$ tem $r$ arestas de retorno, sejam $u u_{1}, \ldots, u u_{r}$ e $w w_{1}, \ldots, w w_{r}$ as arestas de retorno com extremos em $u$ e $w$. As arestas de $F$ serão $v u_{1}, \ldots, v u_{r}, v w_{1}, \ldots v w_{r}$ para cada folha $v \in F\left(T_{F}\right)$; as arestas da árvore não estão contidas em $F$. Tem-se que $u$ e $w$ têm os mesmos ancestrais em $G$, que são os vértices do caminho mínimo em $T_{G}$ entre a raiz e $v$. Se $u$ e $w$ atingissem um mesmo ancestral $y$, tem-se que $(u, v, w, y)$ seria um $C_{4} \mathrm{em} G$. Dessa forma, todas arestas $v u_{1}, \ldots, v u_{r}, v w_{1}, \ldots v w_{r}$ são distintas e toda folha em $F\left(T_{F}\right)$ envia $2 r$ arestas de retorno em $F$.

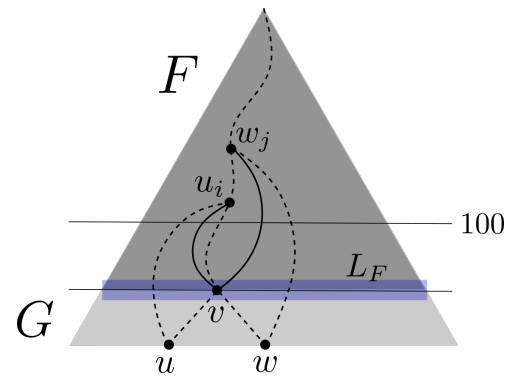

Figura 6.23: Obtenha $F \in T B(100,2 r, g / 2-1)$ a partir de um $(2,1, g)$-grafo $G$.

Tendo definido $F$, afirma-se que ele não possui circuito de comprimento menor que $g / 2$ (Figura 6.24). Tome um circuito $C=\left(x_{1}, v_{1}, x_{2}, \ldots, x_{k}, v_{k}\right)$ em $F$ de comprimento $2 k$, onde $v_{i}(i \in[k])$ são folhas de $T_{F}$, já que toda aresta de $F$ tem alguma folha como um de seus extremos. Para cada $v_{i}$, temse, em $F\left(T_{G}\right)$, os vértices $u^{i}$ e $w^{i}$ que são os dois filhos de $v_{i}$ em $T_{G}$. Pela forma que foram definidas as arestas de $F$, tem-se, em $G$ e sem perda de generalidade, que $x_{i}$ e $x_{i+1}$ são ambos atingidos por $u^{i}$, ou que $x_{i}$ é atingido por $u^{i}$, enquanto $x_{i+1}$ é atingido por $w^{i}$. Assim, o segmento $\left(x_{i}, v_{i}, x_{i+1}\right)$ pode ser substituído por $\left(x_{i}, u^{i}, x_{i+1}\right)$ ou $\left(x_{i}, u^{i}, v_{i}, w^{i}, x_{i+1}\right)$, que são caminhos em $G$. Como duas folhas $v_{i}$ e $v_{j}$ em $C$ são sempre distintas, tem-se $u^{i} \neq w^{i} \neq u^{j} \neq w^{j}$. Fazendo a substituição para cada folha de $C$, tem-se um circuito $C^{\prime}$ em $G$ de comprimento no máximo $\left|C^{\prime}\right| \leq 4 k$. Como $\operatorname{cin}(G) \geq g$, tem-se $4 k \geq\left|C^{\prime}\right| \geq g$, portanto $2 k \geq g / 2$ e $\operatorname{cin}(F)>g / 2-1$.

Para se ter $F \in T B(100,2 r, g / 2-1)$, é preciso encontrar $x^{\prime} \geq 100$ e $L_{F}$ como na definição de $T B(x, r, g)$. Como $g>102$, as arestas de retorno de $G$ devem atingir níveis acima de 101 na 
árvore $T_{G}$. Como $T_{F}=T_{G} \backslash F\left(T_{G}\right)$, então as arestas de $F$ atingem níveis acima de 100. Como toda folha em $F\left(T_{F}\right)$ envia $2 r$ arestas para níveis acima de 100, então o conjunto $L_{F}$ é o conjunto $F\left(T_{F}\right)$, que tem tamanho $\left|L_{F}\right| \geq 2^{-100 / 4}\left|F\left(T_{F}\right)\right|$ e pode-se tomar $x^{\prime}=100$.

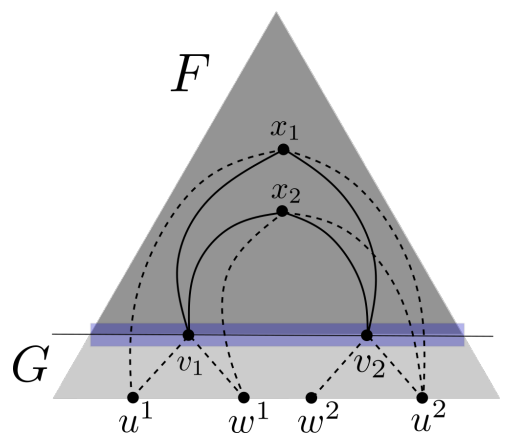

Figura 6.24: Um circuito de tamanho $2 k$ em $F$ induz um circuito de tamanho no máximo $4 k$ em $G$.

Como $F \in T B(100,2 r, g / 2-1)$, existem $\ell^{\prime} \geq \ell(100,2 r, g / 2-1)$ e um conjunto $A_{F, \ell^{\prime}} \in F\left(T_{F}\right)$ não vazio, tal que toda folha em $A_{F, \ell^{\prime}}$ envia pelo menos uma aresta para um nível acima de $\ell^{\prime}$. Dessa forma, a altura de $T_{F}$ é maior que ou igual a $\ell(100,2 r, g / 2-1)$. Como $T_{G}$ tem um nível a mais que $T_{F}$, tem-se que $m(2, r, g) \geq 1+\ell(100,2 r, g / 2-1)$.

Afirma-se que $m(d, r, g) \geq m(2, r, g)$. De fato, a partir de $G$, um $(d, r, g)$-grafo $(d \geq 2, r \geq 1$, $g \geq 4)$ com árvore $T_{G}$, pode-se obter $G^{\prime}$, um $(2, r, g)$-grafo com árvore $T_{G^{\prime}}$, que esteja contido em $G$ e tal que a altura de $T_{G^{\prime}}$ seja igual à de $T_{G}$. Primeiro, toma-se uma subárvore de $T_{G}$ que seja binária, completa e que tenha a mesma altura; ela é $T_{G^{\prime}}$. Depois, toma-se as $r$ aresta de retorno para cada folha de $T_{G^{\prime}}$. Assim, todo valor que é altura de alguma árvore de um $(d, r, g)$-grafo é também altura de alguma árvore de um $(2, r, g)$-grafo. Dessa forma, vale que a menor altura de uma árvore de um $(d, r, g)$-grafo é maior que ou igual à menor altura de uma árvore de um $(2, r, g)$-grafo. Pelos Teoremas 6.3 .10 e 6.3.11, tem-se o seguinte resultado.

Corolário 6.3.12. Sejam $d \geq 2, r \geq 1$ e $g>102$ inteiros, vale que $m(d, r, g) \geq 2 \uparrow\left\lfloor\log _{2}(g / 2-1)\right\rfloor 2 r$.

Lembre-se que $\mathcal{T}_{p, k, g} \in \mathcal{G}_{p, k, g}$ é obtido a partir de um $(k-1,(p-1)(k-1)+1,2 g)$-grafo. Assim, tem-se o seguinte resultado.

Teorema 6.3.13. Sejam $p \geq 2, k \geq 3$ e $g>51$ inteiros, vale que

$$
\left.\left|\mathcal{T}_{p, k, g}\right| \geq 2 \uparrow \log _{2}(g-1)\right\rfloor 2(p-1)(k-1)
$$

Demonstração. Como observado em (6.1), o número de vértices em $\mathcal{T}_{p, k, g}$ é o número de vértices do $(k-1,(p-1)(k-1)+1,2 g)$-grafo auxiliar menos o número de folhas. Pode-se tomar um grafo auxiliar cuja árvore tenha altura mínima $h=m(k-1,(p-1)(k-1)+1,2 g)$. Assim,

$$
\begin{aligned}
\left|V\left(\mathcal{T}_{p, k, g}\right)\right| & \geq \sum_{i=0}^{h-1}(k-1)^{i}=\frac{(k-1)^{h}-1}{k-2} \geq h \\
& \left.=m(k-1,(p-1)(k-1)+1,2 g) \geq 2 \uparrow \log _{2}(g-1)\right\rfloor 2(p-1)(k-1)
\end{aligned}
$$

Em (6.10), foi utilizado que $x^{a}>a x(a \geq 2, x \geq 3)$, tomando $a=h$ e $x=k-1$. Em (6.11), foi utilizado o Corolário 6.3.12. 
Assim, para o caso particular em que se busca um grafo com cintura e número cromático grandes, tem-se uma cota inferior Ackemanniana dada pelo seguinte resultado.

Corolário 6.3.14. Sejam $k \geq 3$ e $g>51$ inteiros, vale que $\left|\mathcal{T}_{p, k, g}\right| \geq 2 \uparrow\left\lfloor\log _{2}(g-1)\right\rfloor 2(k-1)$.

Comparando-se os resultados dos Corolários 6.3.5 e 6.3.14, tem-se, para $k \geq 3$ e $g>51$, que

$$
2 \uparrow\left\lfloor\log _{2}(g-1)\right\rfloor 2(k-1) \leq\left|\mathcal{T}_{2, k, g}\right| \leq(k-1) \uparrow^{2 g-1}\left(6^{k}+1\right) .
$$

As cotas superior e inferior obtidas não permitem localizar corretamente em qual nível da hierarquia de Ackermann está o número de vértices $\left|\mathcal{T}_{2, k, g}\right|$. Elas também não são suficientes para se concluir que a construção de um grafo com cintura e número cromático grandes a partir de árvores aumentadas garante mais ou menos vértices que as construções de Kř́rž ou por amalgamação.

Em [3], prova-se que o número de flechas de Knuth da cota obtida para a altura mínima de um $T B\left(x, r, 2^{s}\right)(x \geq 100, r \geq 1)$ grafo está correto. Não é claro se a cota inferior ou superior estaria mais próxima do valor de $\left|\mathcal{T}_{p, k, g}\right|$. 


\section{Capítulo 7}

\section{Considerações finais}

Obter demonstrações construtivas para a existência de grafos com certas propriedades é uma questão natural e que fornece uma compreensão estrutural desses grafos. Em particular, a existência de grafos com cintura e número cromático grandes é um resultado importante em Combinatória e Teoria dos Grafos, que mostra que nem sempre restrições locais garantem certo comportamento global. As construções apresentadas conseguem grafos com cintura e número cromático grandes, mas para isso, utilizam muitos vértices; é a forma que se tem de contornar a restrição local de cintura grande.

Seguem uma breve recapitulação do que foi visto e alguns problemas relacionados. O Capítulo 2 tratou das provas probabilísticas da existência de grafos [12] e hipergrafos [13] com cintura e número cromático grandes. A demonstração probabilística dada por Erdôs foi marcante, pois além de utilizar um método que estava surgindo, respondeu à questão não óbvia de que existem grafos esparsos com número cromático grande. Antes dessa demonstração, o melhor que havia sido encontrado eram grafos de cintura pelo menos 6 e número cromático alto, os grafos de Tutte.

No Capítulo 3, foram apresentadas as construções iniciais de grafos livres de triângulos e com número cromático grande (Tutte [37], Zykov [39] e Mycielski [28]). Também foram descritas outras construções mais recentes desses grafos, que surgiram em outros contextos (Kneser [19], grafos de shift [15] e de planos projetivos [11]). As diferentes construções apresentadas fornecem grafos cuja ordem do número de vértices varia desde polinomial a fatorial (Teorema 3.7.1).

Nos capítulos seguintes, foram apresentadas construções para o caso geral da cintura. O Capítulo 4 tratou da construção de Kř̌iž [23] para grafos com cintura e número cromático grandes, sem envolver hipergrafos. O Capítulo 5 tratou da construção de Nešetřil e Rödl [33], cuja ideia é combinar grafos a hipergrafos através da amalgamação para conseguir cintura e número cromático grandes. Ela também pode ser utilizada para se obter hipergrafos com cintura e número cromático grandes. No Capítulo 6, apresentou-se uma construção mais recente, de Alon, Kostochka, Reiniger, West e Zhu [4]. Ela obtém hipergrafos com cintura e número cromático grandes a partir de árvores aumentadas e não envolve outros hipergrafos. Todas essas construções possuem muitos mais vértices que o garantido pela demonstração probabilística. Os resultados obtidos foram os seguintes.

Teorema 1.0.1. Sejam $k \geq 4$ e $g>51$ inteiros. Chame respectivamente de $\mathrm{Kz}_{k, g}$, de $\mathcal{A}_{2, k, g}$ e de $\mathcal{T}_{2, k, g}$ os grafos obtidos pelas construçôes de Kř̌ž, por amalgamação e por árvores aumentadas com cintura maior que g e número cromático pelo menos $k$. Vale que 


$$
\begin{aligned}
& 2 \uparrow\left\lceil\log _{2} g\right\rceil(k+8) \geq\left|\mathrm{Kz}_{k, g}\right| \geq 2 \uparrow\left\lceil\log _{2} g\right\rceil(k-1), \\
& (3(k-1)) \uparrow^{g}(4 k+2) \geq\left|\mathcal{A}_{2, k, g}\right| \geq\left(\frac{k-1}{2}\right) \uparrow^{g}(k-1), \\
& (k-1) \uparrow^{2 g-1}\left(6^{k}+1\right) \geq\left|\mathcal{T}_{2, k, g}\right| \geq 2 \uparrow\left\lfloor\log _{2}(g-1)\right\rfloor 2(k-1) .
\end{aligned}
$$

A procura de novas construções, possivelmente com número de vértices menor, persiste. Sabe-se da construção para grafos de Ramanujan [26], que também obtém grafos com cintura e número cromático grandes e com números de vértices como os das demonstrações probabilísticas. Porém ainda seria interessante encontrar uma construção cuja descrição fosse em termos estruturais. Para hipergrafos, existe uma construção com menos vértices que as apresentadas, mas ainda muitos vértices [22]. Essa construção não é totalmente determinística. Ainda é um problema em aberto encontrar uma construção para hipergrafos com cintura e número cromático grandes com número de vértices como o da demonstração probabilística.

Os grafos com cintura e número cromático grandes também inspiram outras investigações. Essa pergunta pode ser feita para outras variantes do número cromático e cintura. Uma variação do número cromático é o número acíclico cromático direcionado. Em um digrafo, esse número é igual ao mínimo de cores para se colorir os vértices sem que haja um circuito direcionado monocromático. Assim, cada classe de cor não pode induzir nenhum circuito direcionado. A existência de digrafos sem circuitos direcionados pequenos com número acíclico cromático grande já foi provada [7]. Temse ainda a seguinte Conjectura 1 de Victor Neumann-Lara que relaciona o número cromático com o número acíclico cromático. A questão é se um número cromático grande garante uma orientação com número acíclico cromático direcionado grande.

Conjectura 1. Existe $g: \mathbb{N} \rightarrow \mathbb{N}$ tal que todo grafo $G$ com $\chi(G) \geq g(k)$ admite uma orientação de suas arestas $\vec{G}$ com número acíclico cromático direcionado maior que $k$.

Outra noção que pode ser interpretada como um tipo de coloração é a de homomorfismo. Num homomorfismo $G \rightarrow H$, cada vértice de $G$ é mapeado num vértice de $H$ e dois vértices adjacentes em $G$ não podem ser levados num mesmo vértice, mas devem ser levados em vértices adjacentes de $H$. Um homomorfismo é como colorir um grafo segundo outro grafo. Um grafo com número cromático no máximo $k$ é homeomorfo a $K_{k}$ e vice-versa. Uma conjectura relacionada é a seguinte.

Conjectura 2. Existe um inteiro $g$ tal que se $G$ é um grafo com grau máximo $\Delta \leq 3$ e $\operatorname{cin}(G) \geq g$, então existe homomorfismo $G \rightarrow C_{5}$.

O que se sabe é que ela não é válida para $C_{11}$ [21], $C_{9}$ [38] e $C_{7}$ [17].

Finalmente, apresenta-se uma última conjectura, de Erdôs e Hajnal [14]. A existência de grafos com cintura e número cromático grandes pode ser vista como um caso particular válido da seguinte conjectura.

Conjectura 3. Para todo $k$ e $g$, existe $f(k, g)$ tal que todo grafo $G$ com $\chi(G) \geq f(k, g)$ contém um subgrafo $G^{\prime} \operatorname{com} \operatorname{cin}\left(G^{\prime}\right)>g$ e $\chi\left(G^{\prime}\right) \geq k$.

Se $G$ fosse necessariamente um clique, a existência de um grafo em $\mathcal{G}_{k, g} \operatorname{com} n$ vértices significaria que $f(k, g)=n$ seria válido. O único caso conhecido é que $f(k, 4)$ existe [36].

Possivelmente, esbarrar com grafos e hipergrafos com cintura e número cromático grandes é algo inevitável ao longo do estudo de Combinatória. Deseja-se que este trabalho torne esses encontros mais especiais. 


\section{Bibliografia}

[1] Wilhelm Ackermann. Zum hilbertschen aufbau der reellen zahlen. Mathematische Annalen, 99(1):118-133, 1928. 8

[2] Martin Aigner, Günter M Ziegler, Karl H Hofmann, and Paul Erdos. Proofs from the Book, volume 274. Springer, 2010. 17, 18

[3] Noga Alon. High girth augmented trees are huge. J. Combin. Theory Ser. A, 144:7-15, 2016. 53,86

[4] Noga Alon, Alexandr Kostochka, Benjamin Reiniger, Douglas B. West, and Xuding Zhu. Coloring, sparseness and girth. Israel J. Math., 214(1):315-331, 2016. 2, 53, 62, 87

[5] Noga Alon and Joel H Spencer. The probabilistic method. John Wiley \& Sons, 2004. 7

[6] J Bárány. A short proof of Kneser's conjecture. Journal of Combinatorial Theory, Series A, 25(3):325-326, 1978. 17

[7] Drago Bokal, Gasper Fijavz, Martin Juvan, P Mark Kayll, and Bojan Mohar. The circular chromatic number of a digraph. Journal of Graph Theory, 46(3):227-240, 2004. 88

[8] Béla Bollobás. Modern graph theory, volume 184 of Graduate Texts in Mathematics. SpringerVerlag, New York, 1998. 6

[9] Karol Borsuk. Drei sätze über die n-dimensionale euklidische sphäre. Fundamenta Mathematicae, 1(20):177-190, 1933. 18

[10] V. Chvátal. The minimality of the Mycielski graph. pages 243-246. Lecture Notes in Math., Vol. 406, 1974. 13

[11] Bruno Codenotti, Pavel Pudlák, and Giovanni Resta. Some structural properties of low-rank matrices related to computational complexity. Theoret. Comput. Sci., 235(1):89-107, 2000. Selected papers in honor of Manuel Blum (Hong Kong, 1998). 3, 21, 87

[12] P. Erdős. Graph theory and probability. Canad. J. Math., 11:34-38, 1959. 1, 6, 87

[13] P. Erdôs and A. Hajnal. On chromatic number of graphs and set-systems. Acta Math. Acad. Sci. Hungar, 17:61-99, 1966. 1, 7, 87

[14] P Erdôs. Some unsolved problems in graph theory and combinatorial analysis. 1971. 88

[15] P Erdos and A Hajnal. On chromatic number of infinite graphs. In Theory of Graphs (Proc. Colloq., Tihany, 1966), pages 83-98. Academic Press, 1968. 3, 15, 87

[16] Joshua E Greene. A new short proof of Kneser's conjecture. The American mathematical monthly, 109(10):918-920, 2002. 17

[17] Hamed Hatami. Random cubic graphs are not homomorphic to the cycle of size 7. Journal of Combinatorial Theory, Series B, 93(2):319-325, 2005. 88 
[18] Shlomo Hoory, Nathan Linial, and Avi Wigderson. Expander graphs and their applications. Bulletin of the American Mathematical Society, 43(4):439-561, 2006. 4

[19] Martin Kneser. Aufgabe 360. Jahresbericht der Deutschen Mathematiker-Vereinigung, 2:27, 1955. 3, 16, 87

[20] Donald Ervin Knuth. Mathematics and computer science: coping with finiteness. Science (New York, NY), 194(4271):1235-1242, 1976. 8

[21] Alexandr Kostochka, Jaroslav Nešetřil, and Petra Smolıková. Colorings and homomorphisms of degenerate and bounded degree graphs. Discrete Mathematics, 233(1-3):257-276, 2001. 88

[22] Gábor Kun. Constraints, mmsnp and expander relational structures. Combinatorica, 33(3):335-347, 2013. 88

[23] Igor Křiž. A hypergraph-free construction of highly chromatic graphs without short cycles. Combinatorica, 9(2):227-229, 1989. 2, 27, 87

[24] László Lovász. On chromatic number of finite set-systems. Acta Mathematica Hungarica, 19(1-2):59-67, 1968. 1, 27

[25] László Lovász. Kneser's conjecture, chromatic number, and homotopy. Journal of Combinatorial Theory, Series A, 25(3):319-324, 1978. 17

[26] Alexander Lubotzky, Ralph Phillips, and Peter Sarnak. Ramanujan graphs. Combinatorica, 8(3):261-277, 1988. 4, 88

[27] Lazar Aronovich Lyusternik and Lev Genrikhovich Shnirel'man. Topological methods in variational problems and their application to the differential geometry of surfaces. Uspekhi Matematicheskikh Nauk, 2(1):166-217, 1947. 18

[28] Jan Mycielski. Sur le coloriage des graphes. Colloq. Math., 3:161-162, 1955. 3, 14, 87

[29] Jaroslav Nešetřil and Vojtěch Rödl. On a probabilistic graph-theoretical method. Proceedings of the American Mathematical Society, 72(2):417-421, 1978. 7

[30] J Nešetřil. K-chromatic graphs without cycles of length $\leq 7$. Comment. Math. Univ. Carol, $7(3): 373-376,1966.27$

[31] Jaroslav Nešetřil. A combinatorial classic - sparse graphs with high chromatic number. In Erdôs centennial, volume 25 of Bolyai Soc. Math. Stud., pages 383-407. János Bolyai Math. Soc., Budapest, 2013. 5

[32] Jaroslav Nešetřil and Jiři Matoušek. Invitation to discrete mathematics. Oxford University Press, 2009. 21

[33] Jaroslav Nešetřil and Vojtěch Rödl. A short proof of the existence of highly chromatic hypergraphs without short cycles. J. Combin. Theory Ser. B, 27(2):225-227, 1979. 2, 27, 37, 87

[34] Rózsa Péter. Konstruktion nichtrekursiver funktionen. Mathematische Annalen, 111(1):42-60, 1935. 9

[35] Raphael M Robinson. Recursion and double recursion. Bulletin of the American Mathematical Society, 54(10):987-993, 1948. 9

[36] V Rödl. On the chromatic number of subgraphs of a given graph. Proceedings of the American Mathematical Society, 64(2):370-371, 1977. 88 
[37] WT Tutte. A three-colour problem. Eureka, 9:352, 1947. 3, 11, 87

[38] Ian M Wanless and Nicholas C Wormald. Regular graphs with no homomorphisms onto cycles. Journal of Combinatorial Theory Series B, 82(1):155-160, 2001. 88

[39] Alexander Aleksandrovich Zykov. On some properties of linear complexes. Matematicheskii sbornik, 66(2):163-188, 1949. 3, 12, 87 